\title{
The Impact of 'Race', Culture and Ethnicity on the Practice of White, British Educational Psychologists
}

\section{Musrat Ashraf}

Professional Doctorate in Education and Child Psychology

School of Psychology

University of East London

August 2016

A thesis submitted in partial fulfilment of the requirements of the University of East London for the Professional Doctorate in Educational and Child Psychology 


\section{Declaration}

University of East London

School of Psychology

Professional Doctorate in Educational and Child Psychology

This work has not previously been accepted for any degree and it is not being concurrently submitted for any degree. This research is being submitted in partial fulfilment of the requirements of the Professional Doctorate in Educational and Child Psychology.

This thesis is the result of my own work and investigation, except where otherwise stated. Other sources are acknowledged by explicit references in the text. A full reference list is included in the thesis.

I hereby give permission for my thesis, if accepted, to be available for reading and for inter-library loans, and for the title and summary to be made available to outside organisations.

Signed

Musrat Ashraf

August 2016 


\section{Abstract}

One criticism of the increased 'racial', ethnic and cultural diversification of British society is the oft-repeated narrative in the British media about educational services being under enormous strain as they grapple with the influx of immigrant children moving into Britain. However, with figures indicating that children of immigrant parents, particularly those from minority ethnic backgrounds, face profound challenges in academic attainment and their school experiences compared to their non-BME peers, the changing demography of schools appears to be a matter of increasing importance for educational policy makers.

With a significant number of the UK school population composed of those from linguistically, ethnically and culturally diverse backgrounds, it is unlikely for Educational Psychologists (EPs) not to encounter pupils who differ from them in terms of 'race', ethnicity and culture. However, with attitudes and perspectives of a culturally diverse population likely to be quite different from that of the helping professional, EPs must be adequately prepared to address the challenges presented when working with those from ethnically, 'racially' and culturally diverse backgrounds. Yet, herein lies the predicament. Since EPs rely on theoretical frameworks that are conventionally derived from a privileged, White Eurocentric/North American middle-class value system, the social, emotional and cognitive needs of other ethnic groups are likely to be neglected, as definitions of normality and abnormality may differ considerably. As a result of this, the need to become culturally competent as EPs has never been more important.

On account of the researcher's reflexivity on being a trainee from a minority ethnic background and, the majority of research into multicultural competence originating in North America, using a social constructionist stance, this study set out to explore the impact of 'race', culture and ethnicity on the practice of White, British EPs.

Twelve EPs across three London local authorities were recruited to participate in the research and data collected via focus groups and semi-structured interviews. 
Data was analysed using thematic analysis which identified five final themes as impacting on EPs' abilities to provide services to those from minority ethnic backgrounds; challenges of working with minority ethnic parents, the culture of schools, changes to education services, EP training courses and reflections on their role when working in a multicultural society.

The implications of these findings for parents, schools, services, EP training courses and EPs are considered. In addition, the impact of this research on the researcher is also addressed. 


\section{Acknowledgements}

This thesis has been a journey- a lengthy and transformational journey, which would have been neither attempted, nor accomplished, without the direction, assistance and support of many people. I would like to take this opportunity of acknowledging those who have been instrumental throughout this process: $\mathrm{Dr}$ Tina Rae and her heartening encouragement for me to pursue this topic, $\mathrm{Dr}$ Laura Cockburn for the many frank and honest discussions we had about life, for being a sounding board for my frustrations and for introducing me to the EP 'Race' and Culture Forum, to the EPs within the 'Race' and Culture Forum for making me realise I was not alone and, all the teaching staff within the UEL EP doctorate training programme for providing words of reassurance and praisethank you all. Last but not least, I owe my deepest gratitude to Dr Mark Fox; a beacon of knowledge who took me under his wing and made me realise that the best, most inspiring and worthwhile paths, cannot be discovered without getting lost- several times. I remain indebted to him for getting me past many hurdles whilst on this journey.

To my wonderful UEL friends; thank you all for promoting the worldview that some journeys can lead to new beginnings, without ending and can lead to beautiful adventures! Thank you, also, to my non-UEL friends who provided an assortment of welcome treats and distractions when I took myself too seriously! To my family- your prayers got me through!

My heartfelt thanks, also, to the hardworking, dedicated and passionate EP participants for their precious time, honesty and openness in making this work possible.

Above all, as a person of faith, I express my sincere gratefulness to God, almighty; whose infinite blessings and bounties in my life cannot be denied and, whose perfect words in the glorious Quran, have been a constant source of refuge and strength. 


\section{Dedication}

This thesis is dedicated to two of the most important people in my life.

My late mother; IB, whose insistence on the importance of Education! Education! Education preceded that of Tony Blair by decades and, whose love, tenderness, care and wisdom made me the person I am today. I miss you, Ammi.

And, my darling husband; HAA, whose strong arms held me up when I struggled, whose beautiful words provided immense comfort and reassurance when I doubted myself and whose countless late-night readings and editing skills ensured that this work would reflect the efforts gone into it and be the best it could.

Dearest $\mathrm{H}$, the depth of my gratitude for the profound patience you demonstrated during this period, knows no bounds. You have been my greatest supporter, biggest comfort and strongest motivation and I am eternally beholden to you for your unwavering help, prayers, support and guidance.

I love you more than you will ever know. 


\section{Contents Page}

Declaration $\quad$ i

Abstract ii

Acknowledgements iv

Dedication V V

Contents Page vi vi vis

\section{Table of Contents}

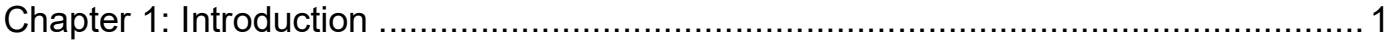

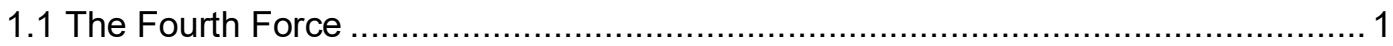

1.2 Race, Culture and Ethnicity, BME, Diversity and White .................................... 2

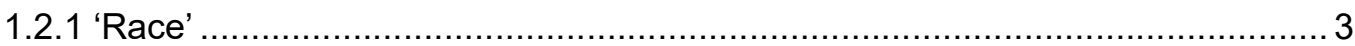

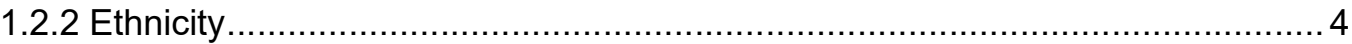

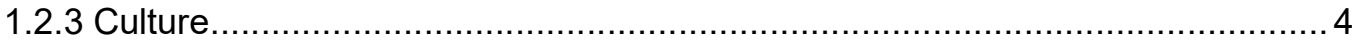

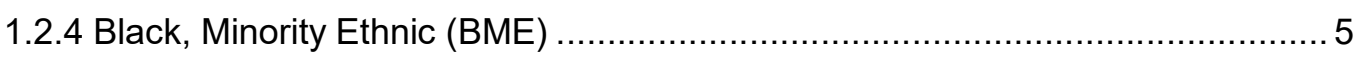

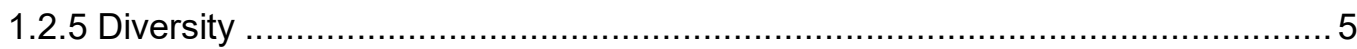

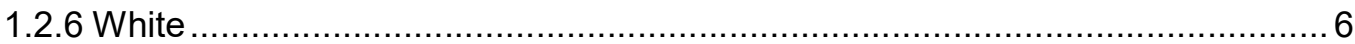

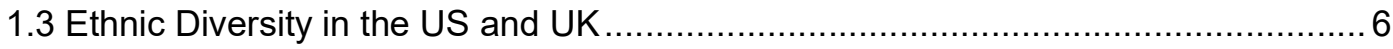

1.4 Multiculturalism and Educational Attainments ................................................ 8

1.5 Multiculturalism and Educational Experience ............................................... 9

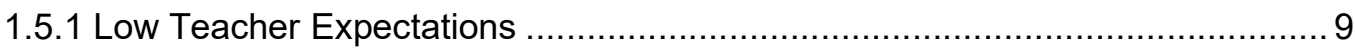

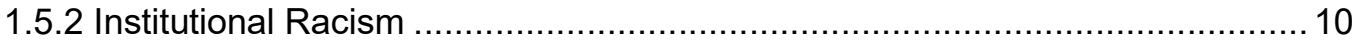

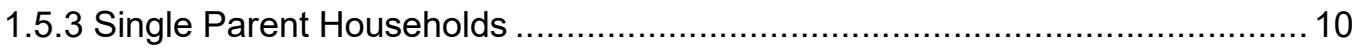

1.5.4 Struggles with Identity and Mental Health .............................................. 11

1.6 The Importance of Reflections in Research...................................................... 11

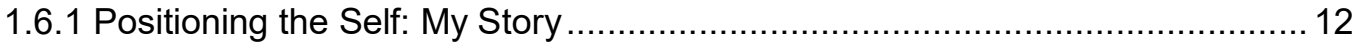

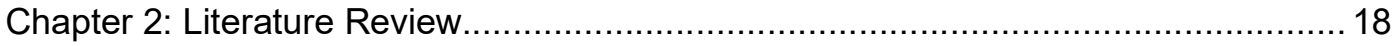

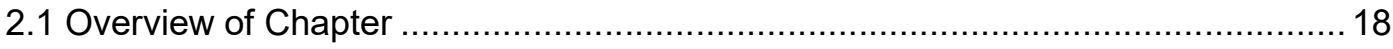

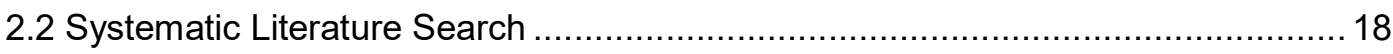

2.3 The Need for Multicultural Competence in Psychology ...................................... 19

2.4 Multicultural Competence in Educational Psychology...........................................20

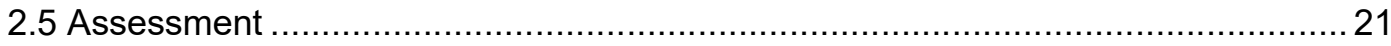

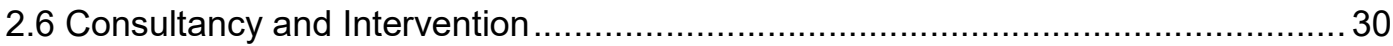

2.7 Need for Linguistic and Ethnic Diversity in School Psychology Personnel............. 32

2.8 Gaps in UK Multicultural Competence and Educational Psychology Literature...... 35

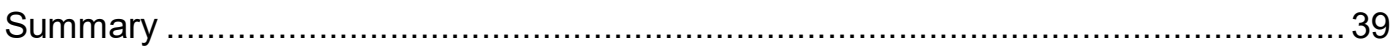




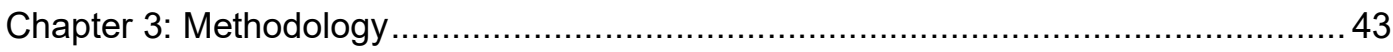

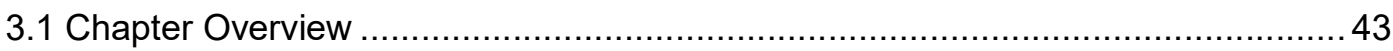

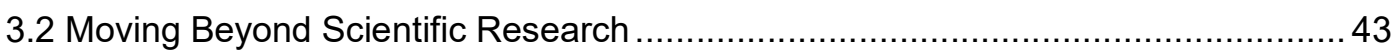

3.3 Ontological and Epistemological Considerations ............................................. 45

3.4 Constructionist versus Social Constructionist Approaches to Race, Culture and

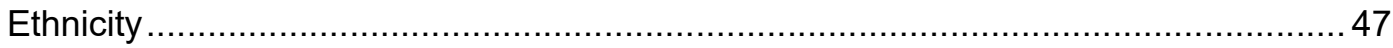

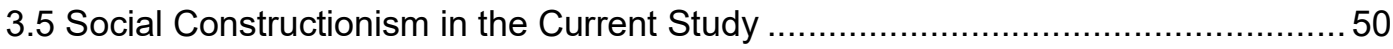

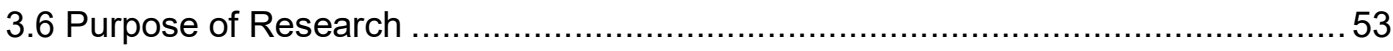

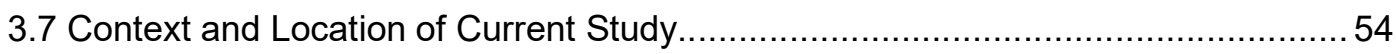

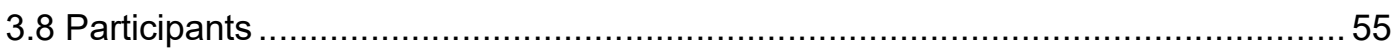

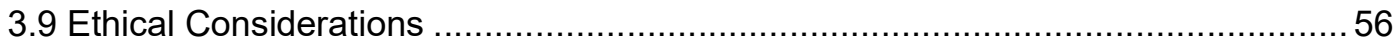

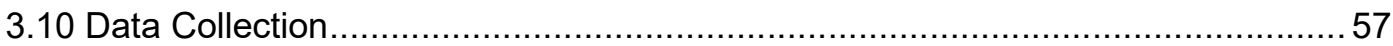

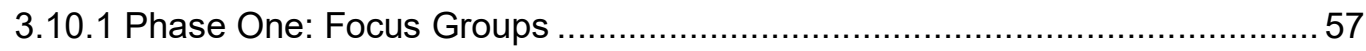

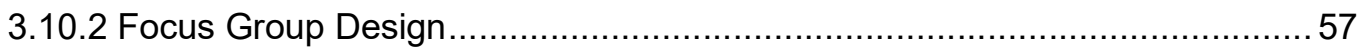

3.10.3 Focus Group Procedure .................................................................. 58

3.10.4 Phase Two: Semi-Structured Interviews .................................................. 59

3.10.5 Semi-Structured Interviews Design....................................................... 59

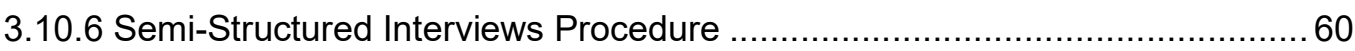

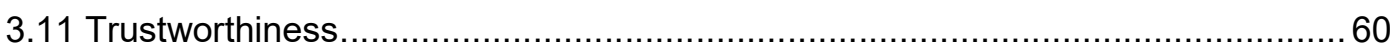

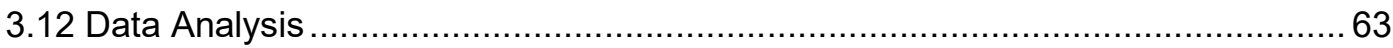

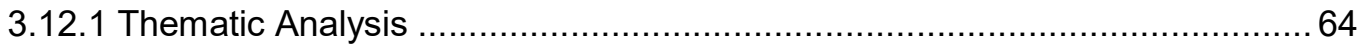

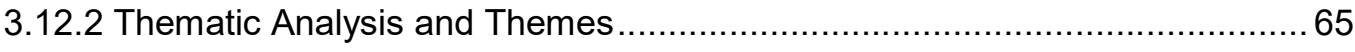

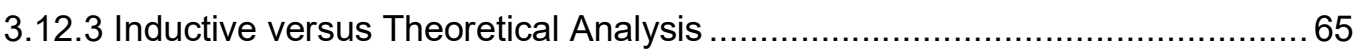

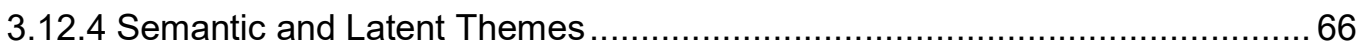

3.12.5 Essentialist/Realist versus Constructionist Thematic Analysis .....................67 67

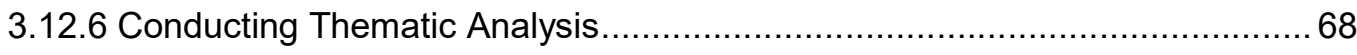

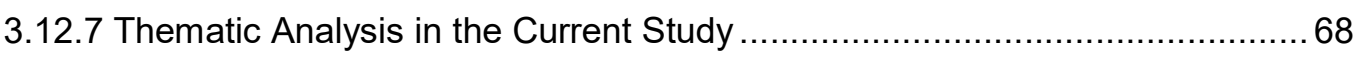

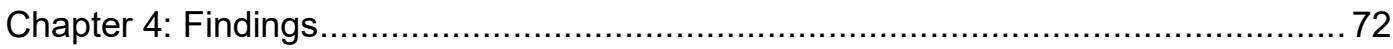

4.1 Final Theme 1 - Challenges of working with BME Parents ............................... 77

Sub-Theme 1.1 Differing Norms of Parenting, Learning and Achievement among

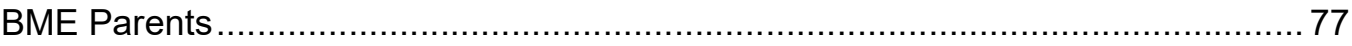

Sub-Theme 1.2 Defensiveness/Disengagement of BME Parents .......................... 79

Sub-Theme 1.3 Barriers of Communication ...................................................... 85

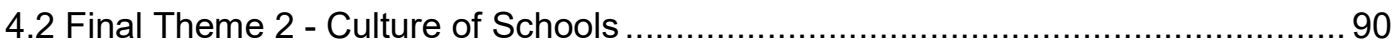

Sub-Theme 2.1 Negative Attitudes towards BME CYP, Parents and Communities . 90

Sub-Theme 2.2 Schools Reflecting Dominant Narratives in Society...................... 91

Sub-Theme 2.3 Negative Attitudes between BME Communities ........................... 91

Sub-Theme 2.4 Uniformity valued over Diversity in Schools................................ 92

Sub-Theme 2.5 Staff Negative Stereotypes and its Effects on EPs Relationship with Schools 
Sub-Theme 2.6 Power Differentials between School and EPs in Tackling Stereotypes

Sub-Theme 2.7 Power of Schools to Determine EP Work .................................... 94

4.3 Final Theme 3 - Changes to Education Services................................................. 96

Sub-Theme 3.1 Cuts in Educational Services and Limited Resources.................... 96

Sub-Theme 3.2 Impact of Service Cuts on EPs................................................. 96

Sub-Theme 3.3 Changing Perception of Diversity .............................................. 98

Sub-Theme 3.4: Changing nature of EP Work................................................... 99

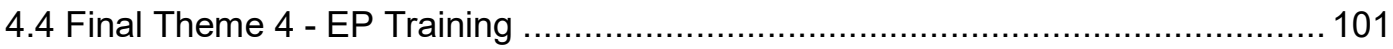

Sub-Theme 4.1 Opportunities to work with diverse communities........................ 101

Sub-Theme 4.2 Opportunities to train with a diverse cohort on EP training

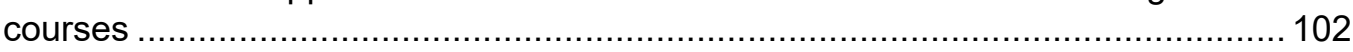

Sub-Theme 4.3 Promoting Diversity within EP Training Staff and Services ........... 103

Sub-Theme 4.4 Training on diversity within EP courses .................................... 105

Sub-Theme 4.5 Need for shift in training curriculum ........................................ 106

4.5 Final Theme 5 - EPs Role Working in a Multicultural Society ............................ 107

Sub-Theme 5.1 EPs Approach to issues around 'Race', Culture and Ethnicity...... 107

Sub-Theme 5.2 Importance of EP Inter-Personal Skills when working with BME

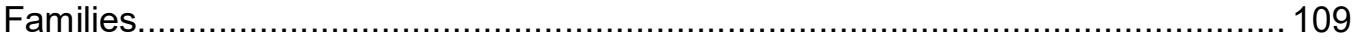

Sub-Theme 5.3 Advocating a Social Justice Approach of working with BME

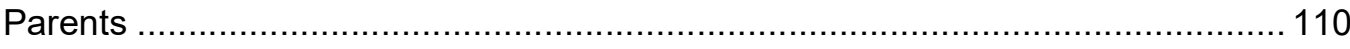

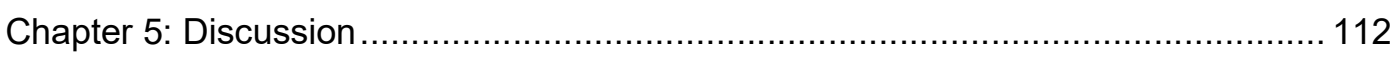

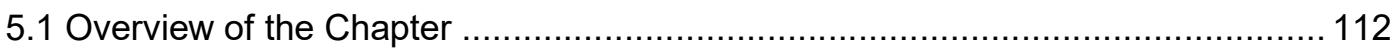

5.2 Final Themes relating to Research Questions........................................... 112

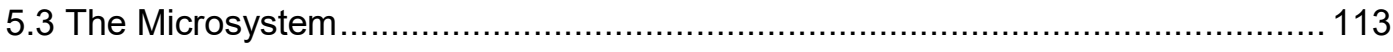

5.3.1 Challenges of working with BME Parents ........................................... 113

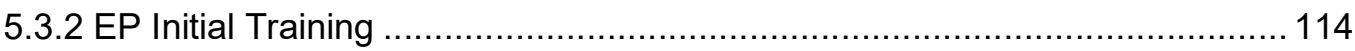

5.3.3 EPs Reflections of Role when working in a Multicultural Society ................ 115

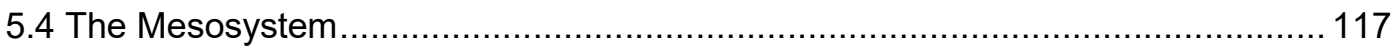

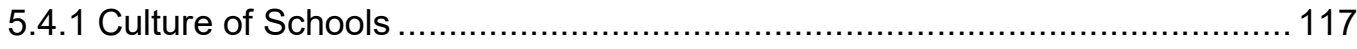

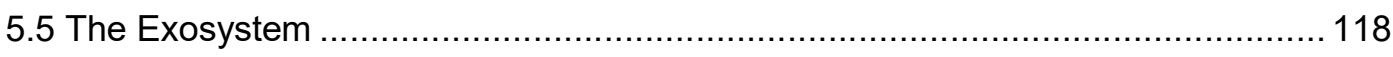

5.5.1 Changes to Education Services ..................................................... 118

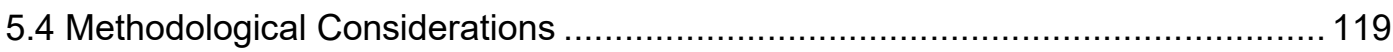

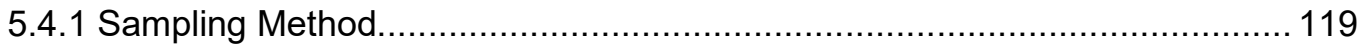

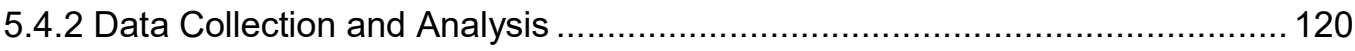

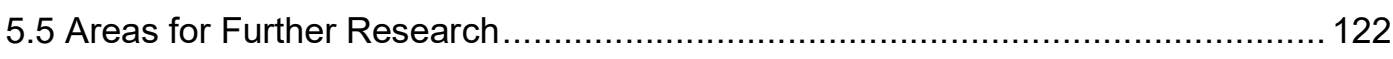

5.6 Implications for Educational Psychology .................................................. 123

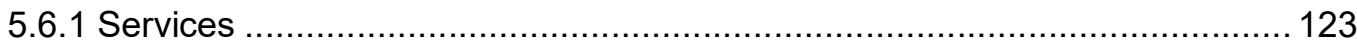

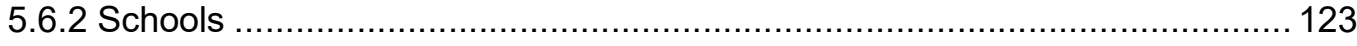

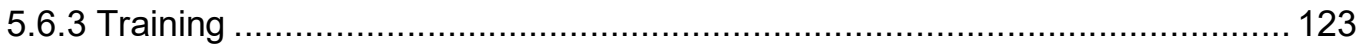




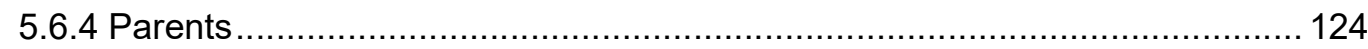

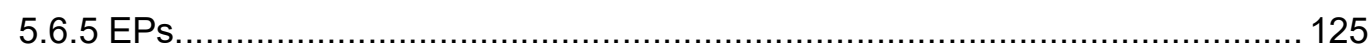

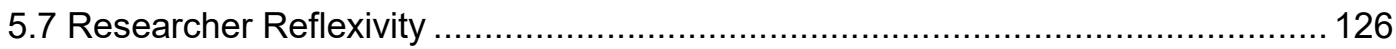

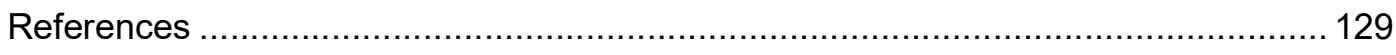

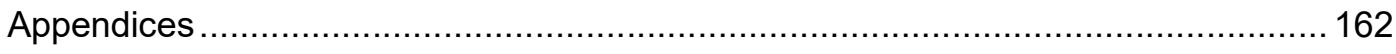

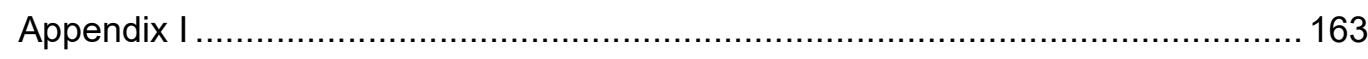

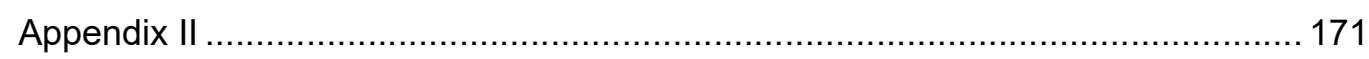

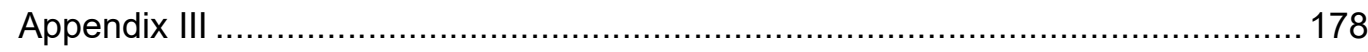

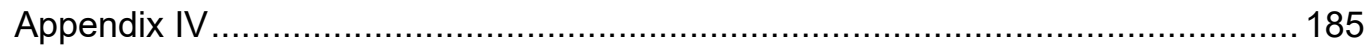

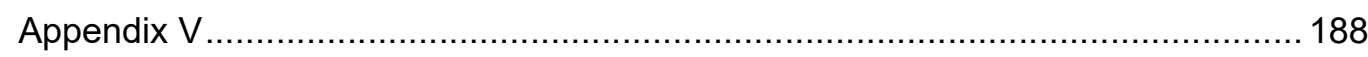

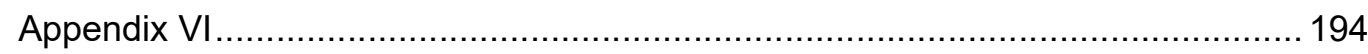

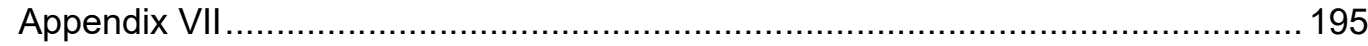

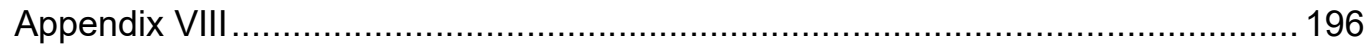

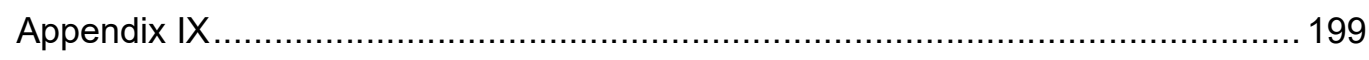

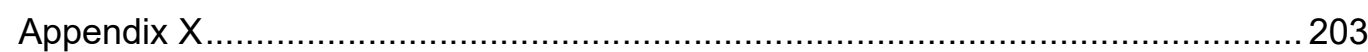

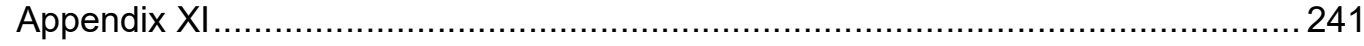

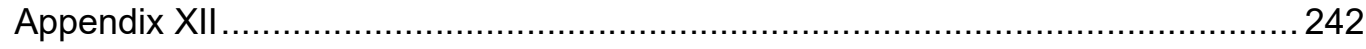

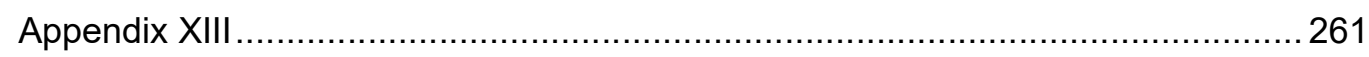




\section{List of Tables and Figures}

Tables

1. Focus Group Characteristics

2. Provisions that may be made by a qualitative researcher wishing to address Guba's four criteria for trustworthiness

3. Phases of Thematic Analysis in the Current Study .59

4. A summary of the themes and sub-themes that developed from the three focus groups on EPs experiences of working with CYP and/or their families from BME backgrounds across three London boroughs

Figures

1. Challenges encountered by white, British Educational Psychologists when working with BME children, young people and their families 63

2. Relationship between the Five Final Themes Identified 105 


\section{Chapter 1: Introduction}

\subsection{The Fourth Force}

The past couple of decades have seen multicultural issues in the delivery of psychological services receiving increased attention and 'culture' being portrayed as a hot topic in psychology. For example, Pederson (1990) states that multiculturalism is the "fourth force" in psychology, which has had as immense an impact to precipitate a paradigm shift in the field of psychology as have psychoanalysis, behaviourism and humanism (p. 93). According to Pederson, the classification of multiculturalism as the fourth force does not replace earlier theories but, instead, complements them:

...the possibility that we are moving toward a generic theory of multiculturalism that recognizes the psychological consequences of each cultural context, where each behaviour has been learned and is displayed...and calls attention to the way in which a culture-centred perspective has changed the way we look at psychology across fields and theories. (Pedersen, 1990. p. xxii)

Pederson further contends that "the main goal of [multicultural psychology] is to convince general psychology that culture is an important contributor to the development of human behavior, and to our understanding and study of it" (Pederson, 1990., p. 6)

While the demographics in some countries, such as the US and in Britain, have become more diverse over the last fifty years, the field of psychology has not changed at a commensurate rate (Ivey, 1995; Pedersen, 2002; Sue, Bernier, Durran, Feinberg, Pedersen, Smith, and Vasquez-Nuttall, 1982). For example, it has been argued that therapeutic practitioners from a non-minority White background belong to the majority White society and, as such, are predisposed to inheriting a cultural conditioning process that instils within them the racial biases of their predecessors (Abelson, Dasgupta, Park and Banaji, 1998; Sue, 2005) that discriminate against clients from non-White majority backgrounds (Sue, Capodilupo, Torino, Bucceri, Holder, Nadal, and Esquilin, 2007). Sue, et al. (1982) further state that there is a "...growing awareness that the human 
service professions, including counselling and clinical psychology...have failed to meet the particular needs of ethnic minorities" (p. 48). Accordingly, ComasDiaz (1998) notes that “...concerns about cultural appropriateness, application of psychological knowledge, issues of inclusion and equal opportunity based on a limited Western European conceptualisation of 'man' are prompting reconsideration and revision of traditional psychological tenets and assumptions" (p. 152)

In fact, race, culture and ethnicity are deemed to be such pressing societal issues that the Society for the Psychological Study of Ethnic Minority Issues (Division 43 of the American Psychological Association) announced its own official journal: Cultural Diversity and Ethnic Minority Psychology, with the hope that it would provide a forum for promoting the cultural and psychological understanding of ethnic minority groups (Comas-Diaz, 1998).

\subsection{Race, Culture and Ethnicity, BME, Diversity and White}

As delineated below, current trends in population growth and immigration suggest that EPs will increasingly work with CYP from minority groups, stressing the need for an awareness of culturally appropriate practices. However, the irony of cross-cultural research is that there appears to be little or no consensus on the use of suitable terminology in the scientific study of 'race', culture or ethnicity. Despite this, certain labels, i.e. 'Black', remain deeply ingrained in scientific discourses (Agyemang, Bhopal and Bruijnzeels, 2005; Hutchinson and McKenzie, 1995), which appear to signify on-going challenges to conducting cross-cultural research: “... research which does not understand or acknowledge the diversity of [different] cultures will always be viewed with suspicion. Moreover, because it is scientifically flawed, it is likely to yield misleading results" (Hutchinson and McKenzie, 1995, p. 701). As a result of this, existing etymological mechanisms are scientifically unsound and ultimately propagate and strengthen the stereotypes, divisions and inequalities of the population of interest, resulting in a weakened ability of practitioners and policy-makers to distinguish and apprehend cultural variances (Hutchinson and McKenzie, 1995). 
It is important, therefore, to consider the codification of 'race', ethnicity and culture, BME and diversity in order to elucidate the comparison and contrast between them, so that their use amidst the context of the current research study is conspicuous to the reader. The term white will also be defined.

\subsection{1 'Race'}

The term 'race' refers to the notion that humans share distinct, inherited and permanent biological characteristics, such as skin colour, hair texture, facial features and stature (Barkan, 2012; d'Ardenne and Maharani, 1999). However, with a lack of evidence of 'race' belonging to a biological category:

With the vast expansion of scientific knowledge in this century, however, it has become clear that human populations are not unambiguous, clearly demarcated, biologically distinct groups. Evidence from the analysis of genetics (e.g., DNA) indicates that most physical variation, about $94 \%$, lies within so-called racial groups. Conventional geographic "racial" groupings differ from one another only in about $6 \%$ of their genes. This means that there is greater variation within "racial" groups than between them...

...Physical variations in any given trait tend to occur gradually rather than abruptly over geographic areas. And because physical traits are inherited independently of one another, knowing the range of one trait does not predict the presence of others. For example, skin color varies largely from light in the temperate areas in the north to dark in the tropical areas in the south; its intensity is not related to nose shape or hair texture. Dark skin may be associated with frizzy or kinky hair or curly or wavy or straight hair, all of which are found among different indigenous peoples in tropical regions. These facts render any attempt to establish lines of division among biological populations both arbitrary and subjective.

(American Anthropological Association (1998)

Some have felt it necessary to situate the word 'race' in inverted commas to highlight that 'race' reflects the social construction; historical and locally prescribed ways of thinking, seeing and talking, which are rooted in the racist history of colonialism, slavery and apartheid (Durrheim, Hooks and Riggs, 2009). 


\subsubsection{Ethnicity}

Ethnicity refers to the mutual social, cultural, and historical practices, stemming from common national or regional backgrounds that make sub-groups of a population different from one another. Equally, an ethnic group is a sub-group of a population with established and shared social, cultural, and historic experiences; with relatively distinctive beliefs, values, and behaviours; and with some sense of identity of belonging to the subgroup (Fernando, 2002). Perceived thus, the terms ethnicity and ethnic group avoid the biological inferences of the terms race and racial group (Barkan, 2012; Jenkins, 1986). However, similar to 'race', the importance attached to ethnicity illustrates that it, too, is in many ways a social construction, and that one's ethnic membership has important consequences for how one is treated (Barkan, 2012). In particular, history and current practice indicate that it is easy to become prejudiced against people with different ethnicities from our own, e.g. the horrors of Nazism, ISIS, ethniccleansing and civil wars raging across the world.

\subsubsection{Culture}

Moving away from ethnicity's focus on group identity, culture is more concerned with group practice, values and beliefs (Schein, 1990) and considered to be perpetually fluctuating and metastasising. As such, culture is notoriously difficult to define (Spencer-Oatey, 2012). However, according to Ferraro (1998) culture is associated with social groups, in that:

Culture is shared by at least two or more people, and of course real, live societies are always larger than that. There is, in other words, no such thing as the culture of a hermit. If a solitary individual thinks and behaves in a certain way, that thought or action is idiosyncratic, not cultural. For an idea, a thing, or a behavior to be considered cultural, it must be shared by some type of social group or society. (Ferraro, 1998. 16)

Given this definition, as almost everyone belongs to a number of different groups and categories of people at the same time, people unavoidably carry several layers of mental programming within themselves, corresponding to different levels of culture, e.g. regional, ethnic, religious, gender, social class and/or linguistic affiliations (Hofstede 1991), which suggests that everyone is 
simultaneously a member of several different cultural groups and thus could be said to have multicultural membership (Spencer-Oatey, 2012).

\subsubsection{Black, Minority Ethnic (BME)}

The term 'Black' has political connotations and refers to those with African and Caribbean origins, who have a shared history of European colonialism, neocolonialism, imperialism, ethnocentrism and racism (British Sociological Association [BSA], 2005). In addition, the Commission for Racial Equality (2007) indicates that the term 'ethnic minority' refers to individuals who, according to the ethnic monitoring categories used in the 2001 National Census, selected an ethnic category other than 'White'. However, it has been argued that the use of 'ethnic minority' overlooks or ignores the ethnicity of majority groups (BSA, 2005.) and that 'minority ethnic' as a term should be used instead as it places the emphasis on the minority status rather than the ethnicity, whereas ethnic minority places the emphasis on the minority status of the group.

For the purposes of the current study, the term Black will be used to refer to individuals with African or Caribbean origins and 'minority ethnic' will be employed in order to refer to those who are visually distinctive from the majority white group.

\subsubsection{Diversity}

According to Ortiz and Flanagan (2002) diversity denotes "...any individual or group whose background and experiences differ significantly from that reflected by the mainstream" (p. 339). Though the term represents a broad range of differences, for the purposes of this study, diversity/diverse will primarily represent racial-ethnic differences. 


\subsubsection{White}

The term White refers to individuals with European ancestral origins who identify, or are identified, as White. The term is serves to distinguish these groups from those groups with visible differences in skin colour (e.g. Black, Asian, etc.), and hence derives from the concept of race but is used as an indicator of ethnicity (Bhopal, 2004). Individuals who are white have also been argued to belong to an unnamed, dominant but usually unquestioned cultural space that is neutral and normative (BSA, 2005).

Since the purpose of this research is to capture the experiences of white EPs when working with those whose 'race', ethnicity and culture is different to their own, the terms 'race', culture and ethnicity, BME and diversity- though differentare considered to complement each other (Fernando, 2010) and will therefore be adopted and used interchangeably in this work.

\subsection{Ethnic Diversity in the US and UK}

Despite its many benefits, globalisation has received much criticism and raised many complex and controversial issues, such as immigration. It has been argued that the politicisation of immigration in Britain reveals popular hostility as well as a tendency, among political leaders, to see immigration as an added burden rather than a valuable asset (Simon, 1982).

However, although immigration has shown an increase in the UK in the last decade and even more recently, with refugees and displaced people from wartorn countries seeking asylum in Britain, its scale is still far smaller than that of America (Lucchino, Rosazza-Bondibene and Portes, 2012). For example, the U.S. Census released in 2010 revealed that the population of individuals from minority ethnic backgrounds (i.e. Hispanic and Asian), increased more rapidly than the population of individuals from a White background and, that, within several decades, individuals from minority ethnic groups would become a numerical majority (U.S. Census Bureaux, 2010). This "diversification of the United States" or literally the "changing complexion of society" (Sue, 2003) is nowhere more evident than in schools. In the United States, students from black 
and minority ethnic (BME) backgrounds, (e.g. black, Hispanic, Asian/Pacific Islander, American Indian/Alaska Native) now comprise almost $45 \%$ of those attending public elementary and secondary schools (U.S. Department of Education, National Center for Education Statistics, 2014).

Comparatively, in the UK, the last Census, carried out in 2011, showed that although the population in England and Wales was predominantly 'White' (86\%), the population as a whole had become more ethnically diverse, with $8 \%$ of residents identifying as 'Asian/Asian British' and $3 \%$ identifying as 'Black/African/Caribbean/Black British' (Office for National Statistics, 2011 Census). In addition, $13 \%$ of the total population reported being born outside the UK. Of this foreign-born population, 33\% reported themselves as being 'Asian' and $13 \%$ as 'Black/African/Caribbean/Black British'.

As in the US, these demographic shifts in population have also led to diversity in UK schools. For example, in England, the government's statistical first release (SFR) which contains data from schools and local authorities, states that $30.4 \%$ of all pupils in state-funded primary and $26.6 \%$ of all pupils in state-funded secondary schools were from a minority ethnic background (Department for Education, 2015). It goes on to state the proportion of pupils from minority ethnic backgrounds in state-funded primary and secondary schools as having increased from $29.5 \%$ and $25.3 \%$ respectively since 2014 . In addition, the rise in the proportion of BME pupils also contributed to an increase in linguistic diversity in schools, with figures indicating that $19.4 \%$ pupils in state-funded primary and $15 \%$ of pupils in state-funded secondary schools were either exposed to, or had English as an additional language (EAL) - an increase of $0.7 \%$ since 2014.

It should be noted that although the population of the UK has grown more culturally diverse over the past couple of decades, the majority ethnic group remains 'White British.' However, Census data highlights regional variations in the ethnic breakdown of the population, with individuals from minority ethnic backgrounds making up the majority of the local population in large towns and cities. For example, BME communities outnumbered individuals' identifying themselves as 'White British' for the first time in London, with certain London boroughs (e.g. Newham and Brent) reporting the proportion of BME residents as 
being as high as three times the national average (Census, 2011). This geographical distribution of ethnicities suggests that Britain "...lacks the integration of a truly multi-cultural society" (Upton, 2012, p.2). In fact, far-right groups have frequently attributed these ecological BME 'hotspots' as causal factors for the race riots between Asian and White individuals in Northern towns in 2001, terrorist attacks in London in 2005 and the riots in 2011 by disaffected youth. Despite this; population projections indicate that the UK will follow a trajectory similar to the US with Britain becoming more ethnically diverse by 2051, so that BME communities make up a significant proportion of the population (Wohland, Rees, Norman, Boden and Jasinska, 2010) and, who instead of being concentrated in mostly deprived inner-city areas, become widely dispersed to more affluent areas.

\subsection{Multiculturalism and Educational Attainments}

These demographic changes mean that multiculturalism remains an issue of growing importance for policymakers around the world (Crutchley 1999; James 1995; Roseberry-McKibbin and Eicholtz 1994). In particular in the UK, where just over a quarter of the school population is comprised of pupils from BME backgrounds, those responsible for promoting academic attainment should be adequately prepared to address the challenges presented when working with an ethnically, racially and culturally diverse population. This is still more important when considering, for example, that in comparison to the overachievement of certain BME groups (e.g. Chinese and Indian) in the UK, pupils from Caribbean and African, Bangladeshi and Pakistani backgrounds underperform in schools, compared to their White peers (Gillborn, 2008; Gillborn and Gipps, 1996; Strand, 2009).

These discrepancies in educational attainment are also evident in the early years. For example, analysis of data from the National Foundation Stage profile in 2014 revealed that as in previous years, the percentage of pupils achieving a good level of development and the expected levels in each of the 17 early learning goals continued to vary between different ethnic groups. Here, $59 \%$ of pupils of any black background and $57 \%$ of pupils of any Asian background 
remained the ethnic groups with the lowest proportion of pupils achieving a good level of development respectively. However, when accounting for individual ethnic groups, pupils from a Pakistani background had notably lower than average percentage achieving a good level of development (50\%) with attainment gaps of 10 percentage points (Department for Education [DfE], 2014).

\subsection{Multiculturalism and Educational Experience}

The challenges for BME pupils extend far beyond that of educational attainment alone. They are also more likely, than their non-BME peers to face additional challenges at school and at home. For example, Rollock (2005) found that young Black boys experienced ongoing challenges related to teacher expectations, institutional racism, lack of role models, and peer pressure/culture of antilearning. These are discussed in detail below.

\subsubsection{Low Teacher Expectations}

As Ajegbo, Kiwan and Sharma (2007) state:

...while it is important to understand another person's religion, ethnicity and culture in order to appreciate more fully who they are, it is then simplistic to define them by one of these alone. Stereotyping often goes further than that. Many African Caribbean boys, for instance, feel defined in school just by their blackness; a crude popular definition of what it is to be a Muslim is now developing. (p. 29)

This is linked to BME pupils being more prone than their non-BME peers to experience low teacher expectations and labelling (Sukhnandan and Lee, 1998) which may explain why boys from Caribbean and African minority groups are significantly overrepresented on the special educational needs (SEN) register, particularly with regards to social, emotional and behavioural difficulties (SEBD), disaffection and disengagement from education (Department for Children, Schools and Families, 2008a; 2010a). 


\subsubsection{Institutional Racism}

In addition, the prevalence of institutional racism-"organisational structures, policies and practices which result in ethnic minorities being treated unfairly and less equally, often without intent or knowledge"- has also been identified as a major factor disadvantaging some BME pupils at school (The Stephen Lawrence Inquiry: Macpherson Report, 1999). This may be demonstrated by research that highlights pupils from Caribbean and African backgrounds being more at risk of exclusion than their demographically matched white peers (Eastman, 2011; Strand, 2009). With research further indicating that excluded pupils are four times more likely to finish their education without having gained academic qualifications (Runnymeade Trust, 2012). With findings from the Office of the Children's Commissioner that a Black Caribbean boy is 37 times more likely to be excluded than a girl of Indian origin and 168 times more likely to be permanently excluded if they were eligible for free school meals and had special needs, than a White girl without special needs from a middle class family (Runnymeade Trust, 2012), it is crucial that educational services develop to become more responsive to the specific needs of ethnic minority groups (e.g. Gilborn, 1997; Nieto, 2008).

\subsubsection{Single Parent Households}

The lack of positive male role models in the lives of children, both at home and at school, has been researched extensively (e.g. Abbott, 2010; Collins, 2006; Felson, Liska, South and McNutty, 1994). With almost two thirds of AfroCaribbean (Reynolds, 2010) and many Somali families headed by single mothers (Heitritter, 1999; Rutter, 2004), the affective ties between children and mothers are often greater (e.g. Chant, 2002). However, the absence of fathers, it has been argued, has led to the over-feminised upbringing and underachievement of Black boys (Sewell, 2010). Sewell (2010) argues that without the guidance of a male role model to curb the damaging disposition inherent within all males, young Black boys are more likely to turn to dominant male figures, rampant in gangs and then become socialised into asserting their masculinity through violence. 


\subsubsection{Struggles with Identity and Mental Health}

When exploring identity among recently arrived Somali children, Kahin (1997) found that although most children were not troubled by living in "two different worlds" and learned to switch between two different cultures (Kahin, 1997., p.41), concern was expressed for those children who rejected their identity, perhaps as a result of being Somali and being perceived as a refugee. In addition, it was found that despite parents being eager for their children to 'fit in' in their new environment, Somali parents often took a suspicious and fearful view of what their children were being exposed to at school. Other studies have found that "immigrant Somali adolescents are at high risk for mental health problems due to the unique challenges they face as they attempt to assimilate two very polar cultures into one self-identity" (Scuglik and Alarcon, 2005, p.20). In addition, depression and other mental health problems are also more prevalent among younger Somalis perhaps as a result of poor housing, poverty, trauma and/or a lack of advice/guidance (Jones, 2007).

Cline, de Abreu, Fihosy, Gray, Lambert and Neale, (2002) highlighted the importance of addressing diversity in predominantly white schools. They found that in schools where $4-6 \%$ of pupils belonged to BME backgrounds, many teachers discounted the significance of cultural and ethnic diversity, which often resulted in situations where BME pupils rejected their own cultural and ethnic background and adopted norms and values of their white peers.

\subsection{The Importance of Reflections in Research}

Critics of qualitative research have frequently questioned the lack of scientific rigour and reliability of qualitative methods and have advocated producing context-independent, generalisable knowledge through statistical methods. In his rebuttal to such opponents, Flyvbjerg (2006) emphasises researcher reflexivity as a key component in how qualitative researchers can explicate bias in research into an opportunity to promote insight and understanding of participants' lived experiences. According to Finlay (2003a) reflexivity in research is a "...thoughtful, self-aware analysis of the intersubjective dynamics between researcher and the researched" (p. 5). Finlay (2003a) gives emphasis 
to the process of reflective practice to be intertwined with the research and not applied as an afterthought or on a post-hoc basis. Since the experiences of researchers influence how they address questions related to epistemology, ontology and methodology (Finlay, 2003b), Primeau (2003) argues that reflexivity addresses the subjective nature of qualitative research as a narrative constructed by researchers and "...enhances the quality of research through its ability to extend our understanding of how our positions and interests as researchers affect all stages of the research process" (Primeau, 2003, p. 9). Finlay (2003a) states the importance of the researcher declaring their position in their work and argues that this is an essential preface to the research process. In fact, it has been argued that researchers, who overlook or disregard their own personal experiences and emotions within qualitative research, are, to a certain degree, guilty of being dishonest (Watts, 2008).

Since one's personal and professional beliefs, norms, values and experiences have led to the exploration of the experiences of EPs working within a multicultural context; in pursuit of reflexivity and in keeping with the social constructionist elements of the qualitative research process, this study will be written in the first person, using "I" and "my", rather than "the researcher" (Webb, 1992).

\subsubsection{Positioning the Self: My Story}

I was born and raised in the North West of England in a former mill-town in Lancashire to Pakistani parents, who had left their native country in the 1960s and made England their home. As highly educated individuals who belonged to families with high social standing, my parents were surprised and immensely disappointed at the lack of work opportunities available to them in their adopted country as well as the social ostracisation they faced at the hands of the local host community. Despite this, my parents worked very hard to attain jobs and to assimilate into the host community's way of life. My father recalls the local Caucasian population gradually moving away and other immigrant families who shared my parents' culture and ethnicity moving in. 
Living within an area that was densely populated by the Pakistani community was wonderful- the clothes, the customs and the languages spoken were the same as the ones I experienced at home but there was very little cross-cultural integration. For example, the primary and secondary schools I attended had pupils that were mostly of my ethnic background. In addition, my friends at school lived locally and their parents were the friends of my parents. I attended inclusive schools with accommodating teachers who went out of their way to get to know our parents and find out more about our culture. I recall classrooms that were filled with objects and artefacts that represented my culture and ethnicity- they were, in essence, a home away from home.

Due to my environment and being surrounded with others from the same ethnic, religious and cultural background; there was very little need, desire, motivation or encouragement to engage with those who were different. I was happy to exist in a safe and familiar world where I would not be challenged in regard to my beliefs, customs or values. At this time, my awareness of diverse cultural groups and the dominant 'white' culture was mainly proliferated through the media and not from personal experience.

From this extreme where I was in the numerical, cultural and ethnic majority and where I had very little requirement or opportunity to interact and engage with those from different backgrounds, I found I was thrown into another extreme when I went into tertiary/post-graduate education. Here, for the first time, I became the numerical and ethnic minority and, I felt, my very visible ethnicity became a challenge for the numerical white majority. I recall people stopping and staring at me when I went out, others avoiding eye-contact with me and, as a trainee teacher, I had some children at the schools I was on placement in, asking me why I had dirty or burnt skin colour! Although I handled this in a stoic manner, I recall feeling very vulnerable at the time. For example, from the safety and security of the environment I had grown up in and where I had become a confident individual, happy in my own skin to suddenly being perceived as being different simply because of the way I looked, was difficult to comprehend. I had grown up hearing my parents advocate the importance of gaining a good education but also telling my siblings and I, that we would have to work harder if we wanted to succeed in a white man's world. I did not pay much attention to this 
until I started university when it became apparent that despite my educational achievements or social interests, I had already been labelled as different and that assumptions had been made of the type of person I was, based on the pigment in my skin.

I felt incredibly challenged at this time; on the one hand I was aware and proud of who I was and the power and privilege of coming from a family such as my own but, I was also aware of how dissimilar I suddenly was in relation to the dominant white community. I detested the judgements people made of me based on my skin colour and their assumptions about a female growing up in my culture- I felt I was hyper-alert to comments related to diversity, immigration and 'all things foreign.' However, I quickly learnt to 'fit in' by developing the capacity to adapt and reconcile myself into a way of life that was greatly disparate to the one I had grown up in. I also acquired language, dress and behaviours that were finely attuned to the sensitivities of the white population.

During this period, I was presented with an opportunity to gain some experience teaching in London, as part of my teacher training course. I recall being taken aback, when I arrived, at the array of ethnicities and cultures I had never come across before. Having gone through life where I thought I fitted in completely to my surroundings, to another where I felt I did not fit in at all, my move to London challenged me further and made me question where I now belonged in this very diverse, vibrant and dynamic city.

However, it was only when I started my Professional Doctorate in Educational Psychology a number of years later, that my journey to discover who I was and how I was perceived by others truly began. A number of seminal events occurred that allowed this to happen. For example, I attended a consultation meeting, with an experienced colleague, to meet with school staff and the parent of a child with alleged learning disabilities. The parent informed everybody that although she acknowledged that her daughter presented with learning needs that were effecting her performance at school, she was reluctant to have her child assessed and formerly labelled as having a learning difficulty as that would lead to cultural ramifications such as her child being seen as a failure by others, which would eventually mean that her daughter would struggle to get married within 
their particular community due to the stigma attached to those with special educational needs (SEN). At this point, my colleague and a member of school staff appeared confused and looked in my direction for verification. Although I had not come across such an objection of a parent's refusal to have their child assessed by an EP, at that particular moment in time; I felt I had to become an advocate for the parent in a roomful of people who did not understand her-myself included- simply because of the fact that she was (incorrectly) perceived as being someone who shared my culture and ethnicity.

In another incident, I was approached by a school to meet with a 'difficult' father who was in 'denial' of his child's learning needs. I was informed that this parent was antagonistic and had a dismissive attitude towards school staff and anyone else in authority. When I met with the parent, he insisted that the school was racist and had negatively labelled his child because he was Black. The child's father refuted the validity of school assessments, the intentions of the staff at school, the racist nature of the country he was living in and the cultural competency of psychologists. The father informed me that he worked as an adult Mental Health Nurse and had seen a lot of the Black patients where he worked as having been labelled as having learning difficulties at school. He felt this was one of the key reasons why they had been incarcerated. He believed England was a racist country that was quick to label BME pupils as atypical and he didn't want that for his child.

I was surprised at times, at the irony of the father calling the children in his child's class racist (“...those people hate coloured people...”), the teachers racist (“... they have an image of Africa and think we're all savages") and the education system racist (“... whenever they don't understand anything a coloured person does, they label him a troublemaker..."), when he himself was making comments to that effect. When I challenged him, I was told that I should know better than to side with "them" because I, myself, was coloured! I felt incredibly powerless and helpless and was surprised at the powerful feelings meetings with this parent would provoke in me. On the one hand, I felt empathy and could somewhat relate to his feelings of marginalisation but on the other hand; I felt frustrated because his child was prevented from receiving specialist intervention due to his fears. I observed that the father felt comfortable talking about issues of 'race' and culture 
when he was with me but the moment a non-BME professional approached, he would become guarded and defensive. I was later informed by a colleague that this father often 'played the race card' in an attempt to end further discussion about his child and that it was easier, as a trainee EP, to do as much as I could and then withdraw, as ultimately, I could not proceed without his permission. As well as feeling upset at hearing this, I was also conflicted about what I should do next- should I make clear to this colleague that I somewhat empathised with the father? That his concerns about his child being labelled because of his 'race' were, to a certain degree, justified? Would I upset my non-BME colleagues/school staff? Would I be 'playing the race card' too? And, should I leave my 'race' out of this?

As well as such experiences with parents, I also felt the issues of 'race', culture and ethnicity emerge amongst my cohort of trainee EPs. For example, there were moments during lectures when we were encouraged to contribute to class discussions and share our experiences. On more than one occasion, I observed that when I mentioned experiencing something different to my peers and drew on my experiences from childhood- the mention of cultural differences resulted in some TEPs looking away and avoiding eye-contact to those who overcompensated and nodded and smiled incessantly. No one, I found, asked me to elaborate or clarify the situation in question. I specifically recall one TEP discussing, with another shocked-looking TEP, how she had visited a school and been told by the teacher that in her class alone, the children spoke 20 different languages. I mentioned that being the norm in major cities like London and the fact that even I spoke 4 languages! She was nudged by another TEP, which resulted in the conversation coming to an end and a change of subject to a more 'neutral' topic.

As assertive and self-assured as I am, I felt denigrated by such incidents and begrudgingly found myself conforming to the thinking and discussions of the majority. I felt lonely, misunderstood and, at times, grew tired of the superficiality of trying to fit in. Going from once feeling completely happy with who I was and what I represented; I felt as though I had been forced into an ethnic and cultural straightjacket that there was no way of escaping from. For the first time as an adult, I felt I was experiencing a crisis in confidence around my identity. I 
questioned myself and reflected whether I would have to discard who I was in order to become an EP; if I would have to force myself to think like a white EP in order to do a good job; if I should 'play it safe' and not raise issues in matters relating to 'race', culture and ethnicity, despite feeling that they were not truly representative of all BME pupils/communities; if I would be seen as a troublemaker; if instead of being complicit in labelling a BME pupil as 'troublesome', I called schools into question regarding their inclusion and diversity policies; whether or not I would be perceived as someone with a 'victim' mentality and, if, I was likely to antagonise others when acknowledging my background as an EP. Most importantly, I wondered how I would be able to provide the most silenced members of society a voice to have them heard in an open and constructive manner, if I, myself, was to remain silent regarding the very fundamentals of my being.

More importantly, I was left wondering that if I, as a trainee EP from a BME background, was left feeling overwhelmed in bringing in my own 'racial', cultural and ethnic identity to the EP training course, then, in a nation that prides itself on its tolerance of multiculturalism, how must others, not from a BME background, accommodate their identities in their interactions with BME peers, colleagues, supervisors and clients? I wanted further clarity on what non-BME EPs experiences of working with individuals from 'racially', culturally and ethnically diverse backgrounds were. I felt that an exploration of their perceptions of what was currently effective or helpful practise as well as what more was needed in the field would help capture both the competencies and constraints that may be linked with the position of being an EP from a non-BME background in the UK. I was interested in establishing in a dialogue to ascertain what personal and professional issues arose in non-BME EPs work related to difference and diversity and to what extent, given the 'politically correct' and 'culturally sensitive' nature and context of their role, they felt they were able to fulfil their role as an EP with those whose 'race', culture and ethnicity differed from their own.

This work is a journey I undertook in an attempt to address these concerns and a cathartic way in which I dealt with my frustrations, feelings of confusion, inadequacy and misplacement. 


\section{Chapter 2: Literature Review}

\subsection{Overview of Chapter}

This chapter explores, in depth, the themes introduced in the previous chapter; the educational under-attainment and experiences of BME pupils within UK schools. To begin with, the reader will be made aware of the systematic, explicit and reproducible measures undertaken to ensure the comprehensiveness of the research review in this chapter. This is followed by a critique of the existing literature pertaining to multicultural competence in psychology and educational psychology and its impact on assessment, consultancy and intervention as a factor on the prevalence and longevity of the educational inequalities experienced by BME pupils in UK schools. Finally, the implications of these findings are discussed in terms of how they helped inform the rationale for this research study.

\subsection{Systematic Literature Search}

A systematic review of both published and unpublished research was conducted using a number of search terms within a range of electronic databases, i.e. EBSCOHOST. Once relevant articles had been identified, their abstracts were read and either selected or rejected (Appendix I).

In addition to the systematic review of literature, a hand search of relevant journals including 'Educational Psychology in Practice', 'Cultural Psychology and Ethnic Minority Psychology', Journal of Cross-Cultural Psychology and 'Educational and Child Psychology' as well as internet searches using Google Scholar were also conducted. Here, as with the systematic review of research, abstracts of papers and relevant articles included or discarded. Finally, references within key articles were also scrutinised as a way of ensuring the most comprehensive literature review possible and either incorporated if deemed appropriate or excluded, if not. 


\subsection{The Need for Multicultural Competence in Psychology}

According to Burden (1997):

Increasingly, important decisions about our educational system are being made by politicians and their representatives on the basis of political dogma or current fashion, rather than the consideration of the available evidence... the need, therefore, for in-depth studies of educational practices, which throw light on a broad range of educational issues, is paramount (p. 13).

Burden further states that "it could be argued that few professional researchers are in a better position than applied educational psychologists to investigate and report upon meaningful and socially relevant issues in education" (Burden, 1997., p. 13).

It has long been argued that the field of psychology has traditionally been Eurocentric, in that, it derives from a White, middle-class value system (Katz, 1985; Smith, 1981) and, as such, it has neglected the social, emotional and cognitive needs of other racial groups and the socio-political injustices they endure on a daily basis (Naidoo, 1996). The demographic changes outlined above indicate the need to move to place multicultural competence at the centre of professionals' work.

Multicultural competence is an area that has received immense empirical and theoretical attention (e.g. Arredondo, 1999; Constantine and Ladany, 2000; ; Ladany, Inman, Constantine and Hofheinz, 1997; Sue, Arredondo and McDavis, 1992). This has largely stemmed from concerns about the disparities between the health status of ethnic minority groups in the US and the cultural bias identified in healthcare judgements and recommendations compared to the dominant White majority group (Sue, Zane, Hall and Berger, 2009). Multicultural competence, as a term, has caused disagreements about its "...necessity, empirical research base, and political implications" (Sue, et al., 2009, p. 525), Vera and Speight (2003) state that:

It is a sure sign of progress that we are no longer reading articles that argue whether diversity is important but instead have a developing body 
of literature that allows for scholarly debate regarding how to integrate multiculturalism into our research, practice, and training (p. 253).

Initially understood to be cultural responsiveness or sensitivity (Sue, et al., 2009), multicultural competence now, in part, means how therapeutic practitioners "...can integrate issues of diversity into their work with racial and ethnic minority clients" (Vera and Speight, 2003, p. 253). In their seminal work, Sue, at al. (1992) state that the two most important characteristics associated with providing effective service delivery to ethnic minority groups involves a) the practitioner being aware of their own cultural/racial origins, including the biases, stereotypes and assumptions that influence their world views and $b$ ) having an awareness of the worldviews of culturally diverse clients. This supports the views of scholars from the multicultural psychology movement (e.g. Pederson, 1999) who propose that it is impossible for psychologists to disregard their own culture or the culture of their clients and that by adopting a multicultural perspective, the understanding psychologists, or practitioners, have of human behaviour would become richer and more meaningful instead of vague and difficult.

\subsection{Multicultural Competence in Educational Psychology}

There have been calls for multicultural competence in the helping professions over the past couple of decades (American Psychological Association, 2003; Sue, Arredondo and McDavis, 1992). The multicultural movement in psychology and education has resulted in increased attention to the importance of multicultural competence for school psychologists (Miranda, 2002). For example, research shows that African-American and Latino students and American Indian students have persistently lower grades and test scores (Ladson-Billings, 2006; Zirkel, 2008) and graduate from high school at half the rate of their more affluent White and Asian-American peers (Greene and Winters, 2005; Orfield, 2004). In addition, those from BME backgrounds are also more likely than their non-BME peers to face addition challenges at school, e.g. experiencing conscious or unconscious teacher biases, an alien and hostile school environment, higher levels of stress and anxiety (e.g. Fueyo and Bechtol, 1999; Gollnick and Chinn, 1991; Rollock, 2005; Sharma, 2005), labelling (Solorzano and Ornelas, 2002), academic disengagement (Barber and Olsen, 2004; Frtizberg, 2001; Little and 
Garber, 2004) and racism, prejudice and stereotyping (Gaine, 2005; Richardson and Miles, 2008; Ullmann, 2009), further impacting their academic performance.

As a result of this, in line with recent literature from multicultural competence, the updated School Psychology: A Blueprint for Training and Practice III notes that:

Addressing diversity is no longer defined simply as acknowledging or being "sensitive" to differences in others. Competence in all aspects of diversity is not demonstrated by an individual's degree of sensitivity to or level of knowledge about a given culture, but rather by the ability to recognize when, where, and how issues of diversity are manifest and operating within the wide variety of activities in which school psychologists engage. When school psychologists are able to recognize the importance of diversity in their practice, they are then empowered and expected to use their knowledge and skills to help schools embrace and address diversity issues effectively at all levels (NASP, 2006, p. 16).

In addition, the document also states that:

Addressing issues of diversity is not an easy task and requires that school psychologists look inside themselves, reexamine their personal world views, and evaluate the nature and extent of their own, often subtle, biases with regard to culture, ethnicity, race, gender, disability, social class, sexual orientation, language, and more. These potential biases and those that may be directed toward the school psychologist by other individuals or agencies will significantly affect the manner in which decisions are made, instruction is developed, behavior is evaluated, interventions are designed, and outcomes are influenced (NASP, 2006, p.16).

\subsection{Assessment}

An area that has received the most attention in the literature on multicultural issues in school psychology is that of assessment (Miranda, 2002), since it is the primary role of school psychologists (Goh, Teslow and Fuller, 1981; Smith, 1984; Stinnet, Havey and Oehlet-Stinnet, 1994) and is an activity that consumes the largest portion of school psychologists' time (Hosp and Reschly, 2002; Huebner, 1993). Assessment encompasses data collection with the purpose of specifying and verifying problems and making decisions about a pupil. However, the assessment process also implies several suppositions, such as the assessor's competence to establish rapport and administer the assessment tool, the 
assumption that the assessment tool is suitably normed for the pupil being assessed and that the assessor has an understanding of the non-verbal language and cultural beliefs and practices of the pupil. In addition, it is also assumed that the pupil recognises and comprehends the expectations of the testing situation and that the assessment tool will accurately represent the pupil's level of functioning (GoPaul-McNicol and Thomas-Presswood, 1998). However, what has transpired is that "...when evaluating individuals with non-Eurocentric cultural influences, many of these assumptions are violated, and they compromise, if not invalidate, the findings and the decision-making process" (GoPaul-McNicol and Thomas-Presswood, 1998, p. 46).

In order to counteract this, when considering the assessment of a linguistically diverse school population, there is a critical need for appropriate and effective assessment to support bilingual children's learning and development (Ochoa, Rivera and Ford, 1997). However, herein lies the problem; the field of education and research lacks the kinds of assessment tools and well-trained professionals required to implement effective assessment practices for this group of children (National Association for the Education of Young Children [NAEYC], 2005). This gap has consequences for children, in that, without appropriate ways to assess young children who have EAL, teachers cannot make the best decisions about how or what to teach. Similarly, professionals such as EPs or Speech and Language Therapists are unable to capture an accurate picture of a child's interests, abilities and learning needs (Winter, 1999). In fact, teachers and other service providers working with bilingual children have expressed concerns over when or what to refer children for and whether to wait or refer bilingual children early on so that they get additional help from relevant services (Broomfield and Dodd, 2004). For instance, the lack of effective tools and practices in assessing bilingual children with special needs has often led to these children being underidentified, resulting in failure to provide much-needed services. In addition, problems with the assessment of bilingual children who are in the process of acquiring EAL sometimes leads to over-identification of special needs. For example, writers of psychological reports, i.e. school psychologists, often do not assess or investigate the possible confounding effects of bilingualism on tests, testing and diagnoses (Figueroa and Newsome, 2006). Here misdiagnosing language delays and other learning and developmental disabilities has resulted 
in children being withdrawn from the classroom to receive services they do not need and thus, missing out on other beneficial activities and experiences (Gutierrez-Cullen and Kreiter, 2003). This over and under representation is referred to as the false positive or the false negative situation (Hall, 1995).

Compounding these difficulties is the enduring stigma and frustration that results when children are mislabelled, which is especially critical with vulnerable children and families who already must cope with multiple challenges (Abedi, Hofstetter and Lord, 2004). As both situations of under and over-identification are undesirable, it is of utmost importance that any assessment approaches utilised have increased validity and reliability of determining language and learning difficulties and/or abilities in bilingual children throughout the preschool and school years (Cline and Shamsi, 2000). It appears however, that although assessment is the most researched and theorised area regarding multicultural issues in school psychology, many practitioners fail to recognise the impact of the discrepancy “....between the culture upon which most standardised tests are based and the cultures of diverse students" (Mewborn, 2005, p.4) and which has continued to contribute to an absence of appropriate and relevant literature to guide and support practitioners in being equipped with the multicultural competencies needed to conduct assessments with culturally and linguistically diverse pupils (GoPaul-McNicol and Thomas-Presswood, 1998; Miranda, 2002).

An area that illustrates this most is that of the use of standardised assessments, particularly with those from culturally and linguistically diverse backgrounds. For example, there is no doubt that language and cognitive ability are inextricably linked (Battle 1998; Schott and Henley 1996). Those that are bilingual have several cognitive advantages over their non-bilingual peers, ranging from aboveaverage intelligence (Peal and Lambert, 1962) in terms of enhanced executive control which facilitates cognitive flexibility and processing mechanisms (Nayak, Hanson, Kreugar and McLaughlin, 1990) and, selective attention (Bialystok, 2001).

Yet, despite these advantages, there is widespread concern about the most effective ways to educate bilingual children as they often enter school with language skills that are unlike those of their monolingual peers (Goldstein, 2004; 
Oller and Eilers, 2002). Children who begin learning a second language at the time of first contact with the educational system are at particular risk for misdiagnosis with language impairment (LI) and other learning and cognitive difficulties (Cline and Frederickson, 2009). For example, the functional demand for learning English for many bilingual children growing up in the United States (the majority of whom are Spanish-English bilinguals), begins when they enter the educational system. This group of children typically begin learning English as an additional language (EAL) between three and six years of age as preschoolers. Here, the amount of time of exposure and specific content of the first and second languages influence acquisition patterns and, as such, the home and school contexts shape the focus of conversations and interactions in different ways (Bedore and Peña, 2008). Thus, children in the early stages of bilingual development are likely to have language skills that are in flux (Genesee, 2004). As the second language gradually becomes more complex, the first language may stall or become less complex (Anderson, 1999). At the same time, the level of development in the second language is not typically comparable to that monolingual speakers of that language. These children are likely to score in the at-risk range on a language and ability assessments in their weaker language. Some children may also score in the at-risk range in their stronger language during this transition to a second language (Mennen, Stansfield and Johnston, 2005).

For many teachers and educational policy makers, the supremacy of English both as the medium of education and as a world language can often make it difficult to appreciate or understand the significance of bilingualism (Gravelle, 1996). As such, when children from linguistically diverse backgrounds are seen to be struggling with aspects of the curriculum, a common assumption may be that there is a problem with communication (Cline and Frederickson, 2009) even though bilingual children are just as likely to have special educational needs (SEN) or learning difficulties (e.g. dyslexia and developmental aphasia) as monolingual children. As a result of this, when bilingual children were thought to have special needs or learning difficulties at school, they were often referred to an EP, who carried out a normative intelligence test and concluded that the child's disabilities were due to a lack of proficiency in the second language (Figueroa, 2002). 
Advocates of standardised assessment (SA) believe that using such tests serve the function of separating the normal population from the disordered population; testing a child to discover their strengths and weaknesses relative to its peers. They would also argue that a numerical score permits statistical analysis which rules out elements of chance in the result and places the child's performance in relation to the performance of its peers (Baker, 2008). However, the argument against this is that an emergent bilingual child at the nonverbal or telegraphic and formulaic stage of acquiring EAL, whose performance is compared to its monolingual English peers of the same chronological age, can only fail and achieve low scores. Here, the child may fail for several years before reaching a similar level as its peers (Duncan, 1989). Subsequently, any language or ability assessments conducted during this stage of development will result in misleading information that underestimates the child's true capacity. Conversely, the child may never reach the same level as its peers depending on how the monolingual assessment and norms were established (Duncan, 1989). Gravelle (1996) states that SAs in any language remain biased in favour of individuals for whom that language is native and, as such, low test scores received by bilinguals are often interpreted as evidence of deficits or even disorders. As a result of this, the language gap in testing has often been a major contributor to the disproportionate numbers of Hispanic bilinguals diagnosed as mentally retarded in need of remedial education when cut-off scores on IQ tests are used (Duran, 1988). In a study of Hispanic children in California, Rueda and Mercer (1985) found that the Hispanic students, who constituted fewer than $10 \%$ of the school population, comprised $32 \%$ of the students identified as mentally retarded. In fact, other data from the study suggest that for over $62 \%$ of the Hispanic students identified as mentally retarded, no symptoms of deficiency were found other than the low IQ test scores (Rueda, 1987 in Duran, 1988).

To counteract this, the use of translation in test adaptation and the development of local norms have commonly been suggested for the assessment of bilingual children (Stow and Dodd, 2003). While these steps have served as the impetus to develop materials in the languages of the local population, there are two main problems with test translation with respect to assessment of learning difficulties. First, translation of language tests assumes that language development in other 
languages follows the same developmental trajectory. Second, translated tests target linguistic forms that are markers of learning difficulties in the source language but omit important morphosyntactic forms, features of vocabulary use and narrative components in the target language that potentially differentiate performance of children with and without learning difficulties (Taylor and Payne, 1983). Lack of normative data on typical bilingual acquisition or early sequential bilingual development further exacerbates current standardised test limitations.

Language development milestones cannot be assumed to follow the same developmental trajectories in the same order across languages. While there are documented similarities in language acquisition across languages at a general level, there are differences in the specific forms that children learn depending on the language(s) being acquired. In language test development an example can be found in the first adaptation of the Preschool LanguageScale-3 (PLS-3) from English to Spanish, which was essentially a translation. An analysis of the translated items from the English and Spanish versions of the PLS-3 demonstrated that such items had different difficulty levels (Restrepo and Silverman, 2001). For example, prepositions were easier in English than in Spanish, but object functions were more difficult in English than in Spanish. The question of appropriate targets for identification of learning difficulties is particularly important when developing assessment tools for bilingual populations. Educational Psychologists must use tests to determine whether impairment exists (Restrepo and Silverman, 2001) and thus, when tests are adapted from English to other languages, it is important to note that the adaptations often do not consider markers of learning difficulties for the target language. Thus, adaptations are very likely to have poorer classification accuracy than the original English tests.

The composition of test norms is the second main barrier for appropriate assessment of children learning two languages. Test norms reflect performance of a reference group to which individual children will be compared in order to make a diagnosis (Standards for Educational and Psychological Testing, 1999). Typically, the question asked in order to make a diagnosis of learning difficulties is whether the individual child performs within the range of or significantly below the reference or normative group. If low performance is below that of the 
reference group for reasons other than underlying ability differences, then the test is not appropriate for that particular child. Although much of the research demonstrates that children learning language in monolingual and bilingual environments demonstrate a high degree of similarity, they also demonstrate some cross-linguistic influences in language performance. Thus, bilingual children do not present profiles of acquisition that are like that of two reference groups (one for each language), particularly in their weaker language (Paradis and Genesee, 1997). The appropriate reference group for a bilingual child is other children learning language in the same or a similar type of bilingual language context.

Consequently, normative data should never be applied to a population, other than that from which it was developed, that is; norms developed from English monolingual speakers should never be applied to bilingual speakers of English (Roseberry-McKibbin 1994). It should be understood that normative patterns for bilingual acquisition will be different (for each language spoken by an individual) when compared with monolingual acquisition patterns for those languages (Quinn 2001). It is widely accepted that bilingual speakers do not form a homogeneous group (Gutierrez-Clellen 1999; Nicoladis and Genesee 1997), yet Stow and Dodd (2004) state that a few researchers presenting normative data for bilingual children rarely comment on whether their sample had a sequential or simultaneous pattern of acquisition. They elaborate by suggesting that where authors have attempted to offer bilingual normative data in tandem with monolingual data the quality of that data may vary. For example, The British Picture Vocabulary Scale: Second Edition (Dunn, Dunn, Whetton and Pintilie, 1997), offers normative data developed from 2571 monolingual children over an age range of 3:00-15:08, whilst the normative data offered for bilingual children were developed from 410 bilingual children (who spoke a range of language combinations and about whom the test authors point out there was no information regarding length of attendance at British schools) and only covers the age range 3:00-8:05.

Related to this is how language assessments adapted from English for other language groups are almost always normed on monolingual groups (e.g. adaptation of the Peabody Picture Vocabulary Test- Revised (PPVT-R) to 
Spanish; Test Vocabulario de Imagenes Peabody (TVIP) or, bilingual children are tested in only their home language (e.g. PLS-3). The result is that bilingual children are often inappropriately compared to a monolingual norm. Performance of Spanish-English bilingual children on the TVIP (Dunn, Padilla, Lugo and Dunn, 1986) illustrates this point. In the development and adaptation process of this test, norms were collected for monolingual Mexican and Puerto Rican children (in Mexico and Puerto Rico). Comparisons of a US bilingual sample with the Mexican and Puerto Rican samples indicated a significantly lowered performance (Dunn, 1987). This lowered performance of the bilingual group is likely because the children knew items in their second language but were not also tested in that language. Evidence of such distributed vocabulary knowledge comes from Umbel, Pearson, Fernandez and Oller (1992), who compared bilingual (Spanish-English) first graders' performance on the PPVT-R and the TVIP. Children who were reported to use both languages at home and those who only spoke Spanish at home scored similarly (below the normative mean) on the TVIP. In English, children who spoke English and Spanish at home scored almost one standard deviation below the mean on the PPVT-R. Children who spoke only Spanish at home scored about two standard deviations below the mean for the English version of the test. When they compared performance on the 61 items that the two versions have in common; investigators found that a significant proportion of words (33-41\%) were known only in one language or the other. These results imply that the two single language scores likely underestimated children's vocabulary knowledge.

Duncan (1989) argues that there will always be controversy about the role and usefulness of SA being used with bilingual children as a child performing in a linguistically contrived context will result in artificial and low performance scores. Additionally, these assessments are often standardised on one population, e.g. White, middle class children and used on other population groups which are socio-linguistically different, e.g. working-class children from urban areas. This indicates that the difficulties in assessment of language impairments and/or other learning difficulties in bilingual children are a lack of standardised (or static) test tools that are valid and reliable for that purpose (Cummins, 1984). 
There has been growing interest among EPs in the UK for a substitute to the norm-referenced or static assessments discussed above, that are usually conducted for summative purposes. As such, interest in Dynamic Assessment (DA) as an alternative form of cognitive assessment has grown considerably in the UK in recent years (Deutsch and Reynolds, 2000; Stringer, Elliot and Lauchlan, 1997). In contrast to SA whereby any kind of interaction or assistance during the assessment is considered unacceptable, unfair or even cheating as it is considered as a threat to the reliability of test scores (Haywood and Lidz, 2007), DA adopts a categorically different stance to SA and maintains that important information about a learner's abilities can only be obtained by offering assistance during assessment. Poehner and Lantolf (2005) argue that “...the express goal of Dynamic Assessment is to unify assessment and instruction into a single activity, the goal of which is learner development" (p. 254). Advocates of DA state that when considering the impact of using SA assessments with bilingual children who may have learning difficulties, the outcomes are not promising as the tests are laden with cultural bias. For example, for tests to give a true picture of a child's speech and language skills, it is assumed that the child is familiar with the objects and pictures they are being asked to work with (Wyatt 1998): cultural differences mean this may not be so. Thus, pictures and printed materials themselves may be unfamiliar to a child from a home where the language spoken has no written form. Instead, they argue that good instruction is associated with higher student outcomes, regardless of the type of language spoken (Wenglinsky, 2000). This is clearly evident in studies with EAL students with learning difficulties (Echevarria, Short and Powers, 2003).

As it is often difficult to differentiate between those who manifest low functioning because of cultural difference and those who have experienced cultural deprivation (Elliot, 2000), DA offers an alternative which, according to Tzuriel (2001) “...is superior over the static approach not only for its differential diagnostic value, but also for its potential prescriptive remediation of deficiencies and enhancement of learning processes" (p. 213). Furthermore, on a broader socio-historical scale, DA can “...open opportunities for investigating cognitive change processes as a function of specific mediational procedures, implementation of learning contexts and use of mental operations across cultures" (Tzuriel, 2001, p. 214). 
However, despite DAs inherent attraction, compared to SA, it remains relatively underused and there is little evidence to suggest that DA approaches are widely employed by EPs in the UK (Deutsch and Reynolds, 2000; Elliot, 2000).

\subsection{Consultancy and Intervention}

In addition to assessments, a significant amount of school psychologists' time also involves conducting consultations with staff and parents and, carrying out individual or group therapy sessions (Hosp and Reschly, 2002; Huebner, 1993). The consultation approach rejects the notion of a hierarchical authority where tensions are more likely to arise and advocates an approach where the consultant and consultee are collaborative and the focus is on the consultant to enable the consultee to solve their own problems (Larney, 2003). Some models of consultation (i.e. Wagner, 2008) may be considered overarching therapeutic frameworks (Cording, 2001) since they draw upon theories that provide a means of understanding individuals by encouraging their participation in comprehending, envisaging and promoting change (e.g. Personal Construct Theory, Kelly, 1955). However, it has been argued that the provision of consultation and therapeutic services do not meet the needs of everyone, particularly those from BME communities. This is because traditional psychological theories addressing pathology are grounded in a value system that is incongruent with that of individuals from culturally diverse backgrounds as it often disregards the influence of cultural variables on development (Canino and Spurlock, 2000; GoPaul-McNicol and Thomas-Presswood, 1998). For example, Western treatment approaches are often monocultural and ethnocentric in the assessment, diagnosis, and treatment of racial/ethnic minority populations (Sue, 2003) and focus on disclosure and the examination of interpersonal, emotional and intrapsychic conflicts. As such, the practitioner's version of normality and abnormality, what constitutes mental health, and intervention strategies are often culture-bound (Sue, 2003). Subsequently, in regard to the provision of consultation and therapeutic interventions in culturally diverse schools; these approaches may not be appropriate for all pupils (Mewborn, 2005). 
Ingraham (2000) argues that although the consultation literature provides some excellent models for delivering indirect, preventative and problem-solving psychological services in schools and for researching the efficacy of school consultation services, the attention to cultural issues in school consultation is currently limited in the literature. In fact, the need for multicultural models and practices has been long advocated within school psychology (Henning-Stout and Brown-Cheatham, 1999) as well as the fields of psychology overall (American Psychological Association, 1993; Hall, 1997). As a result of this, several approaches and models have been suggested to explore the practice of multicultural and cross-cultural consultation in schools, e.g. the Multicultural School Consultation (MSC) which consists of five components (i.e. consultant learning and development, consultee learning and development, cultural diversity in consultation constellations, contextual and power influences and methods to support consultee and client success) and offers a conceptual framework to explore structures, processes, and interrelationships among consultation members (Ingraham, 2000); a conceptual model of multicultural consultation that aims to address the structural and relational issues that may develop when working with culturally diverse students and their families (Rogers, 2000) and identifies key cross-cultural consultation competencies as: understanding one's own and others culture, developing cross-cultural communication and interpersonal skills, examining the cultural embeddedness of consultation, using qualitative methodologies, acquiring culture-specific knowledge and understanding and skill in working with interpreters; and, others that have attempted to identify individual differences within and between cultural groups that are at the heart of multicultural models and which must be considered within relevant systemic contexts, e.g. awareness/sensitivity of the consultant, the ability to consider various frames of reference and procedures to implement these effectively into empirically validated conjoint/behavioural consultation models (Sheridan, 2000) to ensure the successful delivery of consultation services.

However, with the field of counselling psychology having been at the forefront of engaging in multicultural research and theorising (Lopez and Rogers, 2001; Rogers and Ponterotto, 1997), several models of multicultural competence that guide school psychology, in the US, have arisen from work that began in the 
counselling field. One that has had the most influence is the Sue, et al (1982) framework of multicultural competence (Miranda, 2002; Rogers, 1998), which suggests that multicultural competence is composed of multicultural awareness, knowledge, and skills. According to Miranda (2002), the influence of this framework has been such that it is evidenced in definitions of multicultural competence proposed in school psychology literature. The only multicultural competence assessment instrument that delineates the competencies needed by school psychologists in the provision of counselling services to culturally diverse clients and to develop an exploratory measure of multicultural counselling competence in school psychology practice, is the Multicultural School Psychology Counselling Competency Scale (Rogers and Ponterotto, 1998) and is based on the competencies proposed in the Sue, et al. model (1992). Despite this; the Multicultural Counselling Inventory (Roysircar, Taffe, Gutkin and Wise, 1994) is the instrument most used when assessing the impact of multicultural training experiences on school psychologists' multicultural counselling competence (Mewborn, 2001; Velez, 2002).

Therefore, when conceptualising multicultural competence in school psychology using models from the field of counselling psychology (e.g. Sue, et al, 1992.), it should be noted that these models were developed for practitioners whose primary role is counsellor, whereas psychologists working in schools have many roles, only one of which is counsellor. In addition, the Sue, et al. (1992.) framework was developed primarily for practitioners working with adults (Lopez and Rogers, 2001). Thus, competencies addressing some of the special situations encountered by psychologists working with students and their families are not incorporated in this model, rendering them one-dimensional and as failing to acknowledge the complexity and multidimensionality of the role of the psychologist working in schools.

\subsection{Need for Linguistic and Ethnic Diversity in School Psychology Personnel}

Despite assessments with linguistically diverse students being a topic of controversy in the USA since Diana vs. California State Board of Education in 
1970, in which the court decreed safeguards when assessing pupils from linguistic minority backgrounds (Ochoa, Rivera and Ford, 1997), it appears that there is a shortage in the bilingual workforce to provide psychological services to the growing linguistically diverse student population. The need for bilingual school psychologists is demonstrated in the fact that despite 10.8 million pupils in US public schools speaking a language other than English at home, with $25 \%$ of these individuals experiencing difficulties doing so (US Department of Education, 2008), only $11 \%$ of National Association of School Psychologists (NASP) regular members are fluent in a language other than English (NASP, 2008). In addition, of the 30 languages reported by the NASP members, Spanish (48\%), French (13\%) and American Sign Language (8\%) were identified as the top three.

With few school psychology programmes offering training to work with this population (Loe and Miranda, 2005; Ochoa, Rivera and Ford, 1997), this situation is confounded further. For example, in examining the degree to which school psychology programmes delivered training in bilingual psychoeducational assessment, Ochoa, Rivera and Ford (1997) found that school psychologists who conducted bilingual psycho-educational assessment did not believe that they had received adequate training, with nearly $70 \%$ of the respondents describing their training with respect to knowledge of cross-cultural issues involved in bilingual psycho-educational assessment as less than adequate. Additionally, at least $80 \%$ stated that their level of training was less than adequate on the following three competencies: (a) knowledge of second language acquisition factors and their relationship to assessment; (b) knowledge of methods to conduct bilingual psycho-educational assessment; and (c) ability to interpret the results of bilingual psycho-educational assessments. Thus, it can be suggested that school psychologists who do conduct bilingual psychoeducational assessment are providing service in an area in which they have not received adequate training. In fact, when evaluating their school psychology training programmes, school psychologists were able to acknowledge only one programme that provided adequate training in all four competencies, two that provided adequate training in three competencies, and four programs that provided adequate training in two competencies (Ochoa, Rivera and Ford, 1997). 
This is a cause for concern, as highlighted by Curtis, Grier and Hunley (2004) who note that:

The field of school psychology has been largely Caucasian throughout its history... Although individuals from diverse ethnic backgrounds and those fluent in languages other than English continue to be seriously underrepresented in the field, many school psychologists work in settings in which they serve an increasingly diverse student population (p.52).

For example, with $90.7 \%$ of White/Caucasian school psychologists in the USA between 2009-2010 (Curtis, Castillo and Gelley, 2012) serving 52\% of White/Caucasian, $23 \%$ of Hispanic/Latino, $16 \%$ of Black/African American, 5\% of Asian-American/Pacific Islander, $1 \%$ of American Indian/Alaskan and 2\% of the Pre-K to 12 public school population (National Centre for Educational Statistics, 2012), there is a critical need to increase the number of ethnic minority school psychologists (e.g., Clark, Mercer, Zeigler-Hill and Dufrene, 2012; Davis, Mclntosh, Phelps and Kehle, 2004; Fagan, 1988). This is vital as the discrepancies between the ethnicities of school psychologists and the clients they serve may well impair their ability to relate to their clients and provide appropriate services (Yeh, Eastman and Cheung, 1994; Thompson and Alexander, 2006).

In addition, with those from BME communities expressing a preference for therapists to be of the same background (Whaley, 2001) and with data from the NASP for the 2004-2005 school years indicating that only $7.4 \%$ of school psychologists identified themselves as belonging to an ethnic minority group (Curtis, Lopez, Castillo, Batsche, Minch and Smith, 2008), it is crucial that the training of school psychologists focusses more effort on recruiting and retaining applicants from BME backgrounds. However, it appears that school psychology training programmes have lagged behind counselling and clinical psychology training programs in the recruitment of minority students (Fiegener, 2009). Nevertheless, the trainees from BME backgrounds who do make it through to school psychology training courses are reported to frequently undergo a number of challenges, ranging from an absence of culturally similar mentors, limited financial support, insufficient undergraduate preparation and a non-optimal 
institutional climate (Zhou, Bray, Kehle, Theodore, Clark and Jenson, 2004). Racial micro-aggressions; "brief, everyday exchanges that send denigrating messages to people of colour because they belong to a racial minority group... often unconsciously delivered in the form of subtle snubs, or dismissive looks, gestures, and tones" (Sue, Capodilupo, Torino, Bucceri, Holder, Nadal and Esquilin, 2007, p. 273) have also been proposed as race-related experiences which may impact the functioning and engagement of ethnic minority graduate students (Clark, Mercer, Zeigler-Hill and Dufrene, 2012).

With society having become increasingly diverse over time and the demographics of school psychologists remaining relatively unchanged, there is now a need more than ever to ensure that school psychology training emphasises culturally competent practices (Canter, 2006) to counteract the challenges BME students face at school and that have a lasting impact on their educational, financial and emotional outcomes. Further complicating matters is the fact that there currently exists a severe personnel shortage within the field of school psychology, with two thirds of the school psychology workforce likely to retire by 2020 (Curtis, Hunley, Walker and Baker, 1999), negatively effecting the delivery of services in public schools (Curtis, et al., 1999; Fagan and Wise, 2000; Miller and Palomares, 2000). With a burgeoning BME and linguistically diverse school population and declining numbers of school psychologists, the field of school psychology will be under ever-increasing pressure to adequately provide services to an evolving school population.

\subsection{Gaps in UK Multicultural Competence and Educational Psychology Literature}

The Swann Report; Education for All (1985) arose from concerns expressed throughout the 1960s and 1970s about the academic performance of children from the West Indian community. In his preface to the aforementioned report, the Secretary of State for Education and Science at the time, Keith Joseph, stated that:

The government is firmly committed to the principle that all children, irrespective of race, colour or ethnic origin, should have a good education 
which develops their abilities and aptitudes to the full and brings about a true sense of belonging to Britain (no pagination).

He further added that the Committee of Enquiry into the Education of Children from Ethnic Minority Groups “...have done a great service in drawing the issues affecting ethnic minority pupils to public attention." (Swann Report, 1985, no pagination). The report also noted that:

The fundamental change that is necessary is the recognition that the problem facing the education system is not how to educate children of ethnic minorities, but how to educate all children... Britain is a multi-racial and multicultural society and all pupils must be enabled to understand what this means (p. 363)

It is necessary to combat racism, to attack inherited myths and stereotypes, and the ways in which they are embodied in institutional practices... Multicultural understanding has to permeate all aspects of a school's work. It is not a separate topic that can be welded on to existing practices... Only in this way can schools begin to offer anything approaching the equality of opportunity for all pupils which it must be the aspiration of the education system to provide (p. 364)

Despite these assertions, educational inequities for students from BME communities in the UK continue to exist over 30 years after the publication of this seminal report.

There has been some response from the UK EP field relating to the extensive and pervasive disparities that exist in the educational experiences and attainments of BME pupils compared to their White peers, e.g. publication of the Challenging Racism and Inequality in the Education and Child Psychology edition of the Educational and Child Psychology journal (M'gadzah and Gibbs, 1999) which looked at the influence of racism and inequality and cultural bias in the treatment of African-Caribbean males (Rollock, 1999) and the assessment of bilingual children (Usmani, 1999), institutional racism directed towards minority communities (Hathiwala-Ward and Swinson, 1999; Howitt and OwusuBempah, 1999; Reed, 1999) as well as training practitioners to address the cultural, linguistic and special educational needs of children with a multicultural community (Bolton and M'gadzah, 1999; Cline, 1999; M'gadzah, Saraon and Shah, 1999; Verma, 1999). In addition, the follow-up edition on 'Race', Culture and Ethnicity in Educational and Child Psychology (Williams, Weerasinghe and 
Hobbs, 2015) focussed on issues around identity (Abdi, 2015; Hewett, 2015; Sultana, 2015) and the concept of belonging (Cartmell and Bond, 2015; Frehill and Dunsmuir, 2015), multicultural perspectives and perceptions of Autism (Rupasinha, 2015; Theara and Abbott, 2015) and the experiences of BME girls in primary school (Rizwan and Williams, 2015).

However, it seems that the US, which despite its troubled and controversial history of race relations, has substantive research that focusses on acknowledging the salient differences in the educational experiences of those from minority communities and how school psychologists should address these. In contrast, EPs in the UK appear to adopt a rather moderate approach with the publication of intermittent yet specialised literature which focusses on a narrow range of issues that endorse the importance of culturally sensitive practise. To counteract this, there is a fundamental need for there to be a wider conversation around 'race', culture and ethnicity among EPs in the UK and how culturally competent practices can be embedded within mainstream psychology, as they appear to be in the US.

Still, the British Psychological Society (BPS); representative body for psychology and psychologists in the UK states that it "...recognises its obligation to set and uphold the highest standards of professionalism..." (p. 2), and, in so doing:

...respect individual, cultural and role differences, including (but not exclusively) those involving age, disability, education, ethnicity, gender, language, national origin, race, religion, sexual orientation, marital or family status and socioeconomic status (BPS Code of Ethics and Conduct, 2009, p.10).

Similarly, in their Statement of Inclusive Education (2002), the Division of Educational and Child Psychology further states that "...psychologists will demonstrate an acceptance of difference and diversity, in terms of abilities, values and aspirations held in a plural society" (p.2). Here, while making an acknowledgement to diversity, these statements from the BPS and DECP appear simplistic and conceptualise diversity within the context of EPs being reactive, or 'sensitive', to acknowledging the differences of the individuals they support and do not encourage EPs to be proactive in developing and delivering 
support, guidance and services that accurately reflect the needs of a multicultural society. A comprehensive understanding of diversity that stresses the importance of associating with other fields (e.g. health and social) is required.

The little work that does exist in guiding EPs to implement multicultural competence in their work with individuals from BME backgrounds in the UK appears to be drawn from the US. In addition, it must also be noted that although both the UK and US have significantly diverse societies and consequently schools; both countries vary significantly in their minority-ethnic population composition. Furthermore, the US and UK have different histories of racism (Bowling and Phillips, 2003) and its ensuing effects on those targeted, e.g. complex processes of ethnic identity in which the ethnic person is constantly assessing the "fit" between self and the different social systems in the environment (Spencer and Markstrom-Adams, 1990, p. 292 as cited in Sodowsky, Kwan and Pannu, 1995, pp. 135-136). Therefore, caution should be exercised when extrapolating multicultural competences strategies and recommendations from the US literature and generalising it to the UK BME school population.

Moreover, it must be noted that although the patterns of educational inequalities are generally similar across ethnic minority groups (e.g. Black pupils), caution needs to be exercised as "the BME population cannot be viewed as homogenous as the challenges within and between groups vary starkly" (Wells, 2010, p.1). In addition, there also appear to be intra-ethnic differences that exist between those who belong to the same community (e.g. Indian/Pakistani, etc.) thus there are variations, that highlight the need for practitioners to shift away from the etic approach and adopt the emic approach when investigating what is needed to work with individuals from BME backgrounds.

Despite this, it appears that the educational psychologists in the UK are following a similar trajectory to their school psychologist peers in the USA in terms of a shortage of professionals. For example, survey data indicates that there are 2925, predominantly female, educational psychologists of working age (25-64 years) registered with the Health Care and Professions Council (HCPC, 2015) in England, with the majority (487) falling into the 60-64 age category (National 
Collage for Teaching and Leadership, 2016). However, there appears to be paucity in information that highlights how ethnically and/or linguistically diverse the population of EPs are in the UK or, how the current population of EPs feel in delivering services to those from BME or linguistically diverse backgrounds. Despite wide-spread affirmation of the importance of multiculturalism in education and psychology, it seems that multicultural competence in educational psychology in the UK is not receiving sufficient attention in the literature.

\section{Summary}

This review has highlighted that a substantial amount of research looking at issues around 'race', culture and ethnicity in educational psychology originates from the US and that little research has been conducted with the purpose of examining the skills needed for the range of roles educational, or school, psychologists perform (Lopez and Rogers, 2001; Ortiz and Flanagan, 2002), particularly when working with a multicultural, multi-ethnic and multiracial population in the UK.

Research has predominantly focused on the process of assessment related to bilingual children in education who were thought to have learning difficulties. A wealth of research highlights the fact that language skills are necessary with every sort of school curriculum, specifically; for the comprehension and expression of spoken language, for literacy and educational achievement and to interact with peers and adults (Dockrell, Sylva, Huxford and Roberts 2009). However, due to globalisation and an increase in the numbers of bilingual and multilingual children in schools around the world, meeting the needs of these children has often been problematic. For example, it is often the case that those with EAL have inaccurately been labelled as slow learners compared to their monolingual peers. Whist some of these children genuinely do make slow progress at school due to barriers related to acquiring a second language, there are some who will experience severe and continuing difficulties (Cline and Shamsi, 2000). Conventional methods of psychometric assessments have often resulted in errors of identification and have raised concerns over reliability and validity. As a consequence, the need for the Educational Psychology profession 
reduce its over-dependence on the use of these assessments and utilise alternative approaches; carrying out observations at school and at home, jointly working with parents to determine general language development as well as evaluating teaching methods and making use of formative assessments, i.e. DA, in assessing bilingual children who may have learning difficulties has been advocated. However, these methods are not extensively used.

Research has also highlighted the majority of psychologists conducting consultations and providing therapeutic services in schools. These methods, despite being collaborative and allowing exploration of sensitive and/or difficult views, as well as generating detailed information, have been highlighted as not being supportive to those from BME communities. For example, the provision of consultation and therapeutic services requires the practitioner to possess an awareness of the cultural and linguistic background of the client and to determine if the learner has the knowledge and linguistic proficiency required to complete the task and to be cognisant of the fact that their analysis of certain issues may be viewed as an invasion of privacy by individuals from some cultural backgrounds. Furthermore, since psychological models and concepts are deemed Eurocentric in nature (Katz, 1985; Smith, 1981) and have been argued to overlook the social, emotional and cognitive needs of other racial groups and the socio-political injustices they endure on a daily basis (Naidoo, 1996), there is a need for psychologists delivering consultation and intervention services to be mindful of the theories underpinning these practices being incongruent with the value and belief systems of those from culturally diverse backgrounds (e.g. Canino and Spurlock, 2000; GoPaul-McNicol and Thomas-Presswood, 1998). Although there is a need for practitioners to alter their mode of consultation and intervention delivery by using alternative and flexible procedures that support the views and beliefs of those from diverse cultural backgrounds, attention to cultural issues in school consultation is limited (Ingraham, 2000). In addition, the majority of consultation models used in schools originate from the field of counselling psychology and are, thus, one-dimensional, failing to acknowledge the complexity and multidimensionality of the role of the psychologist working in schools. 
Research also identifies a shortage in the bilingual personnel to provide psychological services to the growing linguistically diverse student population. However, confounding the matter further is that limited psychology programmes offer training to work with a bilingual population (Loe and Miranda, 2005; Ochoa, Rivera and Ford, 1997), with the majority of school psychologists conducting bilingual psycho-educational assessments describe their training with respect to knowledge of cross-cultural issues involved in bilingual psycho-educational assessment as less than adequate. In addition, with individuals from BME communities articulating a preference for therapists to be of the same background (Whaley, 2001) but those from diverse ethnic backgrounds and those fluent in languages other than English continuing to be seriously underrepresented in the field of psychology, there is a need now, more than ever, for psychology training programmes to recruit and retain applicants from BME backgrounds. Yet, BME trainees who succeed getting onto school psychology training courses are described as regularly experiencing various challenges, e.g. an absence of culturally similar mentors, limited financial support, insufficient undergraduate preparation and a non-optimal institutional climate (Zhou, Bray, Kehle, Theodore, Clark and Jenson, 2004).

This review has highlighted the extensive use of quantitative methodology in evaluating research on 'race', culture and ethnicity and showed the lack of qualitative data in the study of multicultural competence amongst psychologists working in schools. The limitations of existing research include a high reliance on surveys as a method of data collection (e.g. Bainter and Tollefson, 2003; Figueroa and Newsome, 2006; McCloskey Athanasiou, 2000; O'Bryon and Rogers, 2010; Ochoa, Rivera and Ford, 1997, etc.) which raises issues of generalisability due to small sample size and low response rates as well as concerns around validity due to response bias.

While the literature review presents some important findings and interesting conclusions, there are significant gaps in the current state of knowledge, namely that, with society and consequently schools having become increasingly diverse, the demographics of educational psychologists remains relatively unchanged. As such, there is now a need more than ever to ensure that psychologists working in schools demonstrate culturally competent practices to offset the 
continuing challenges BME pupils face at school. However, despite over a quarter of the school population in the UK comprising of pupils from diverse backgrounds, there is a dearth of research that examines the skills needed for the range of roles EPs perform when working with a multicultural, multi-ethnic and multiracial population in the UK. Similarly, there is paucity in research that documents what challenges, if any, EPs in the UK face when working with pupils and their families who are likely to differ from them in terms of 'race', culture and ethnicity.

With a burgeoning BME school population, there is a gap in the literature on the use of qualitative research methods on eliciting the views of EPs in the UK and exploring how they adequately provide services to an evolving school population that is racially, culturally and ethnically diverse. This research study, carried out from a social constructionist perspective, aims to establish dialogue, awareness and understanding of a previously unexplored area and uses a qualitative approach to collect data through focus groups and semi-structured interviews. Analysis of EPs experiences and their views on the impact of 'race', culture and ethnicity on their practice will be carried out using thematic analysis. The next chapter addresses the methodology adopted in this study. 


\section{Chapter 3: Methodology}

\subsection{Chapter Overview}

This chapter sets out the research theory and methodology used in the current research and how it has guided data collection and data analysis. An outline of my epistemological position and purpose for the study are given, followed by a justification for utilising thematic analysis as a qualitative approach in exploring EPs' experiences of working within a multicultural school context. The reader will then be guided through the processes of participant recruitment, data collection and data analysis, alongside their possible relation to issues of trustworthiness and researcher reflexivity. Ethical considerations, as stipulated by the University of East London's Ethics Committee and the British Psychological Society's Code of Ethics and Conduct, will also be highlighted.

\subsection{Moving Beyond Scientific Research}

Scientific research has been defined thus: "...a systematic process of inquiry consisting of three elements or components: (1) a question, problem or hypothesis; (2) data; (3) analysis and interpretation of data" (Nunan, 1992, p. 3). Further, that it must be conducted systematically, sceptically and ethically (Robson, 2011) and that it must be based on empirical data. For many researchers employing quantitative methods in their investigations, this has come to mean rigorously clinical, transparent and unbiased, value free (or value neutral) research, the results of which, as well as being independent of the researcher or observer, are able to be generalised to a broader population (Robson, 2011). Here, the focus is to operate under the assumption of objectivity, in that, "...there is a belief that there is a reality to be observed and that rational observers who look at the same phenomenon will basically agree on its existence and its characteristics" (Johnson and Christensen, 2010, p.36).

In contrast, for others who subscribe to qualitative methods of research, deeper scrutiny of these rules allows for a much broader scope to scientific investigations (Darlaston-Jones, 2007). For example, there is a general consensus among 
qualitative researchers that "reality is socially constructed" (e.g. Guba and Lincoln, 1989). In that sense, all knowledge is relative and there is a subjective element to all research and knowledge and holistic, ungeneralisable studies are also justifiable (Nunan, 1992). Although a binary distinction, which may appear to be naïve and simplistic, appears to have been made between the two methods, Reichardt and Cook (1979) state that quantitative and qualitative methods are in many ways indistinguishable and that "...researchers in no way follow the principles of a supposed paradigm without simultaneously assuming methods and values of the alternative paradigms" (Reichardt and Cook, 1979., p. 232).

Moreover, although quantitative researchers state methodological objectivity as the foundation of their method, they are unable to recognise the numerous subjective decisions that are made throughout the research process. For example, Onwuegbuzie and Leech (2005) argue that:

...even the process of deciding what to study and what instruments to use follow subjective decisions made by the researcher. Similarly, the selection of measurement items, statistical analysis and alpha levels are influenced by subjective opinions and beliefs about what is the 'best' course of action (in Kempson, 2012, p. 62).

Bryman (1988) adds that although the terms quantitative and qualitative research are usually seen to signify different ways of gathering data, there is a recognition that they are both connected with distinctive epistemological and ontological assumptions. When deciding which research method to use, Bryman (1988) states that one may make choices depending on which approach is best suited to the research question (technical) or by considering broader, salient, questions regarding the nature and practice of science and the generation and legitimation of knowledge (epistemological). Bryman states that "...if the research problem is one which directly emanates from a particular epistemological position then the appropriateness of a research technique is significant, for the technique must properly reflect the epistemological framework in which the research is embedded" (Bryman, 1988., p. 83). Here, regardless of the choice of the research method used, an emphasis is placed on demonstrating inter-connectedness between method and epistemology. 


\subsection{Ontological and Epistemological Considerations}

Individuals' are continuously learning throughout their lives. From both informal and formal education, they constantly interpret new information and evaluate it (Merriam, Caffarella and Baumgartner, 2007) and, from this learning process, come to know and internalise knowledge. In order to determine how one's belief system regarding the nature and acquisition of knowledge may lead one to a particular methodological framework, this section will explore the concepts of ontology and epistemology and identify my ontological and epistemological position in the current study.

Beck (1979) contends that "the purpose for social science is to understand the social reality as different people see it and to demonstrate how their views shape the action which they take within that reality" (Anderson and Bennett, 2003, p.153). According to this view, ontological distinction is a critical facet of the research process because it enables the researcher to uncover how his/her perceptions of human nature impact on the approach he/she consciously adopts to reveal social truths (David and Sutton, 2004). Cohen, Manion and Morrison (2000) argue that there are more than tenuous links between the ways in which one might view reality and the choices one might make in regards to methodological considerations. Bracken (2010), when discussing the importance of ontology and epistemology awareness in practitioner research, further states that it is of utmost importance for researchers "to clearly reflect upon their own ontological and epistemological perspectives and to reflect upon the ways in which these may inform development of appropriate research paradigms" (p. 1).

Bracken (2010.) argues that a research practitioner may espouse divergent ontological perspectives, or ways of viewing social reality. They may adopt the belief that the world of social interactions exists independently of what one perceives it to be; as a rational, external entity and responsive to scientific and positivist modes of inquiry, to one that views social reality as co-constructed by individuals who interact and make meaning of their world in an active way, by searching for truth in people's lived experiences through rigorous interpretation (Graue and Walsh, 1998; Byrne-Armstrong, Higgs and Horsfall, 2001). Although the former tradition has informed the ontological foundations of research for 
some time, particularly in the domain of the physical sciences (Bitter-Davis and Parker, 1997; Gallagher, 2008), Pring (2000a, p. 90) argues that both of these ways of coming to the research are informed by historical, cultural and philosophical backgrounds which have to be addressed explicitly. According to Bracken (2010), without such an examination, a researcher may not become aware of his/her philosophical premises on which arguments are based to justify his/her research processes and findings.

Epistemology, as a branch of philosophy, is concerned with the nature and scope of knowledge (Edwards, 1967). There are various definitions of epistemology, ranging from it being viewed as "a structure in which individuals construe the nature and origins of knowledge, of value and of responsibility in a sequential and logical process" (Perry, 1970, p. 1), to “...a system of beliefs which is composed of more than one belief and the beliefs within the system are more or less independent" (Schommer-Aikins, 2002). According to Morgan (2007), epistemology "....influences how research questions are asked and answered" (p.52).

A number of epistemological positions exist which assert that knowledge is obtained by particular approaches (e.g. constructionist, positivist) to research. As a result of this, several debates have raged between social researchers aligning themselves with a particular research paradigm, i.e. quantitative or qualitative, which has resulted in them taking a particular epistemological position and, thus, believing knowledge is discovered in a particular way and by certain methods (Morgan, 2007). However, Howe (1988) argues that "...a philosophical perspective is only of value to the extent that it helps to shape practice and that helping to do this ultimately requires careful attention to exactly what that practice is" (p.15). Howe also states that researchers should "resist the tyranny of methodological dogma" (p.15.) as a researcher's epistemological perspective is, most likely, to influence the practical components (method) of the investigation undertaken. Their epistemology, therefore, “...has implications for every decision made in the research process" (Mertens, 1998, p.3).

Since the subject matter of the current study relates to 'race', culture and ethnicity, it is important to examine relevant paradigms and positions, in 
particular; social constructionist as adopted by those engaged in the research process. For a detailed explanation of alternative approaches to the study of 'race', culture and ethnicity considered, see Appendix III).

\subsection{Constructionist versus Social Constructionist Approaches to Race, Culture and Ethnicity}

Although constructionism is often used as an umbrella term for both constructionism and social constructionism (McLeod, 1994), there are fundamental underlying differences between the two theoretical stances, which will be discussed briefly.

As with post-positivist approaches which question the homogeneity, singularity, predictability and objectivist principles so highly valued by positivism (Gitlin, 1990) and move, instead, towards a social consciousness of multiple belief systems and multiple perspectives (Gonzalez, Biever and Gardner, 1994), constructionism refers to the process by which the observer creates reality, by giving meaning to what is observed (Jonassen, 1991; Von Foerster, 1984; Von Glasersfeld, 1988; Watzlawick, 1984). Here, reality is constructed through a person's active experience of it. Thus, one cannot access the world objectively as the world in an objective sense cannot be known. According to constructionists, an individual's interpretation or construction is as true as any other individual's interpretation or construction, on the assumption that it works within a particular context (Dickerson and Zimmerman, 1996), thus suggesting that all stories or interpretations that work are equally valid and that no single truth or interpretation exists (Dickerson and Zimmerman, 1996.). With the belief in the existence of many possible 'truths', it is difficult, from a constructivist position, for an observer or researcher to 'criticise' an individual's story or perspective. Therefore, according to the constructivist perspective, the views of individuals who attribute poverty on immigrants 'taking our jobs' (rather than capitalist organisations moving their capital to other, more profitable, countries) as well as the views of those with ideas of racial superiority used, for example, to rationalise the exploitation of minorities, would be just as valid as those on the 
receiving end of racism, who are left feeling weak, oppressed and marginalised simply because of the colour of their skin or the country of their birth.

As constructionism disregards the effects of a dominant social reality that influences the creation of meaning (Held, 1990), social constructionism defines the role that the social and cultural context plays in the manner in which an individual perceives or makes sense of his/her world. The basic tenet of the social constructionist approach is that reality is socially constructed by and between the persons who experience it. Here, reality is considered as a consequence of the context in which the action occurs and is shaped by the cultural, historical, political and social norms that operate within that context and time and can, therefore, be different for each of us based on our unique understanding of the world and our experiences of it (Berger and Luckman, 1966). According to social constructionists, individuals create or construct their own understanding or knowledge through the interaction of what they already believe and the ideas, events, and activities with which they come into contact (Ultanir, 2012). Reality, in this case, “...is completely subjective and need not be something that can be shared by anyone else but at the same time it is independent of the person living it" (Darlaston-Jones, 2007). The constructionist epistemology is consistent with the view that the individual is a sense maker and seeks to understand or make sense of the world as it is seen and experienced. Social constructionism provides a perspective with which to view the world that allows the unique differences of individuals to come into focus while at the same time permitting the essential sameness that unites human beings to be identified (Ashworth, 2003). "This means that it is not necessary for any of us to share the views of others but at the same time none of us can change or alter our reality simply because we might wish to" (Darlaston-Jones, 2007). In this instance, each individual reality is true for the person because he or she experiences it but it is independent of that person due to his or her inability to alter it (Gergen, 1999).

Underpinning all these, Hunter (2002) states that epistemologies “...do not exist outside of the people who construct and use them. Individuals and groups adopt various epistemologies at different points in time to make sense of the world." Hunter further states that epistemologies are "...not equal in status, in society at large, or in the academic community" and that they "... are situated within 
political, historical and economic contexts that can provide power and legitimacy to their knowledge claims" (p. 120). When considering research around the key concepts of the current study relating to 'race', culture and ethnicity, it could be argued that as the concept of 'race' resulted from the Western European colonial project beginning in the fifteenth century (Smedley, 1993; Takaki, 1993), the ideology of 'race'- the idea that humans can be divided into distinct, permanent categories specified by biological characteristics such as skin colour, blood group and hair texture (d'Ardenne and Mahatani, 1999)- was used to justify the exploitation of other people and their lands. As a result of this, 'race' "...remains an ideology of the taxonomy of bodies built on structural inequality" (Hunter, 2002, p. 120). However, whereas biological differences were once purported to be fixed, the ideology of 'race' is plastic and has shifted over time to an ideology that now focuses on cultural differences as the basis for inequality (Hunter, 2002). Despite this, Smedley (1993) states that the differences based on one's 'race' remain a "folk classification" that describe human differences as inherently unequal (p.25). In fact, it has been noted that 'race', culture and ethnicity are "...a social construction that refers to different types of human bodies for the purpose of creating and maintaining a matrix of material and ideological domination of one group by another" (Omi and Winant, 1994).

When considering the experiences of those who belong to a 'race', culture or ethnicity other than those from the dominant group, research from the UK suggests that this group of individuals face a number of educational (Dearling, 1996) and socio-economic (Pehrson, Vignoles and Brown, 2009) inequalities. Similarly, the institutional power differences between the dominant White community and the non-dominant Black community in America and the structural inequalities and discrimination Black people face in the areas of education, occupation and income, can be explained as the dominant White group having developed a racial praxis that allows them to maintain their systemic advantages and to obscure the fact that the system is unfair (Bonilla-Silva, 2009). "In this way, racism is not just an ideology, but a material reality, and a structural system that sorts resources unequally according to 'race'” (Hunter, 2002, p.120). Therefore, from the social constructionist perspective, it could be argued that the experiences of those from dominant, e.g. Caucasian, backgrounds are likely to be inherently different than those from non-dominant, e.g. black or minority 
ethnic, groups. This is in line with the social constructionist belief, in that, an individual's reality is vastly different from that of another as a result of their prior experiences, the socialisation process they were subject to and the cultural differences resulting from their different economic positions (Darlaston-Jones, 2007).

\subsection{Social Constructionism in the Current Study}

Social constructionism appears to be a suitable methodology for the current study as it asserts that individuals are constructed by and are constituents of society and that "knowledge" is acquired by understanding what "knowing is" and the means by which individuals "come to understand what there is to know" (Robson, 2011). For example, although many scholars now argue that 'race' is not "real" in the biological sense, as a concept, 'race' is still very much a sociocultural reality (Hunter, 2002) in that it is a "...non-accidental...structure of domination patterned historically, culturally, socially and economically" by the White majority in Western countries “...in order to keep the minoritised populations residing in those countries in check" (Gillborn, 2008, p. 4). As a result of this, individuals and groups receive "differing amounts of resources according to their ascriptive racial" (Hunter, 2002, p.120), cultural (O'Niell, 2003; AustenSmith and Fryer, 2005) and ethnic (Booth, Leigh and Varganova, 2012) categories.

As the concept of 'race' is guided by the social meaning that is ascribed to it, in that; it is informed by historical, social, cultural, and political values (Guess, 2006), social constructionists state that the content of one's consciousness, including one's mode of relating to others, is taught by the culture and society one lives in. As a result of this, according to the social constructionist approach, "...all the metaphysical quantities we take for granted are learned from others around us" (Owen, 1992, p. 386). From the social constructionist viewpoint, language plays an integral part in connecting individuals and, as such, the focus is not on the individual person but rather on the social interaction, in which language is generated, sustained, and abandoned (Gergen and Gergen, 1991).

Furthermore, Berger and Luckman (cited in Speed, 1991, p. 400) state that 
individuals socially construct reality by their use of agreed and shared meaning communicated through language. Hence, our beliefs about the world are social inventions (Van Niekerk, 2005).

Hoffman (1991) suggests that through ongoing conversation with others in one's social world, one is able to develop a sense of identity or an inner voice. Anderson and Goolishian (cited in Hart, 1995) further add that, as humans, “...we live with each other in a world of conversational narrative, and we understand ourselves and each other through changing stories and self-descriptions" (p.184). As such, social constructionism highlights the importance of normative or, grand, narratives, which are formed by and, in turn, influence people and against which people measure themselves (Van Niekerk, 2005). Grand narratives, states Rapmund (2000), are reinforced by the weight of numbers, tradition and firmly entrenched power structures. White and Epston (cited in Speed, 1991) elaborate and state that “...the particular meanings we impose on behaviour are dictated and organised by whatever dominating analogies or interpretive frameworks are currently available" (p.400). This supports the social constructionist argument that relationships between people are either conforming to or lacking a fit with the idealised roles or ways of relating to others (Owen, 1992). Social constructionists, therefore, focus on knowledge as power, believing that "cultural specifications" exert a real influence on people's lives (Dickerson and Zimmerman, 1996, p.80) and take a stand on the subjugating effect of discourses.

Although social constructionists acknowledge that more than one reality or account of reality exists, they disagree with constructivists in asserting that all stories are not equally valid (Van Niekerk, 2005). They state that some narratives are not respectful of gender, ethnicity, 'race' or religion and caution against the power of singular accounts which are inclined to further silence and marginalise those whose stories do not to fit. Social constructionists value narratives based on an individual's lived experience rather than on expert knowledge and are interested in accounts that honour and respect the community of voices inherent in each individual and how these accounts can be respected within a particular system (Rapmund, 2000). Furthermore, social constructionists state that only when narratives that dominate others are deconstructed and examined, do 
alternative choices become available (Dickerson and Zimmerman, 1996). Social constructionists acknowledge the social nature of human life, while at the same time it encourages individuals to tell their own stories (Van Niekerk, 2005). As social constructionism includes and emphasises many realities and personal narratives, it is considered a significant and appropriate epistemological framework for the current study.

The previous chapter highlights a number of research studies on 'race', culture and ethnicity that have been conducted using positivist epistemology where the researcher has utilised an objective stance in an attempt to highlight issues relating to the topic of diversity in society (e.g. Figueroa and Newsome, 2006; McCloskey Athanasiou, 2000; Bainter and Tollefson, 2003; O’Bryon and Rogers, 2010; Ochoa, Rivera and Ford, 1997, etc.). Social constructionism challenges this view and states that the best way in which to understand an issue is to ask the very people who are faced with it, as they are likely to be in the best position to describe their emotional experiences regarding their specific areas of concern. Here, how they perceive their experiences and not whether their reports accurately reflect 'reality' is what is considered to be of importance whereas the idea of accuracy would be considered judgemental (Van Niekerk, 2005). Furthermore, discourse from anthropology, history and sociology characterises the concept of 'race', as having a modern history (Guess, 2006). In fact, Roy (2001) states that 'race' and its associated terms, such as ethnicity were"...created mainly by Anglo-Europeans, especially English, societies in the 16th and 19th centuries" (p.81) as a belief about the inherent superiority and inferiority of groups based on ethnic distinctions (Gossett 1963; Bernal 1987; Bennett 1988). In addition, with social constructionists' stating that some narratives relating to ethnicity, 'race' or religion are not respectful and can silence and marginalise those whose narratives do not to fit the dominant discourse, it could be argued, when considering the concepts of 'race', culture and ethnicity for the current study, that these "socially constructed features of social organisation" (Guess, 2006, p.654) represent “...historical products of human activity... brought about by the concrete actions of human beings" (Berger and Luckmann, 1966, p.116). Similarly, these are then “...learned by the new generation during the same process that socialises them into the institutional order" (Berger and Luckmann, 1966, p.61). 
As a result of this, social constructionist epistemology in the current study explores the experiences of educational psychologists' when working within diverse settings and, in particular, with pupils, parents and teachers whose 'race', culture or ethnicity may be different to their own. This provides new opportunities for personal meanings and viewpoints to be discovered. As well as providing me with an awareness of the social, academic and emotional context in which educational psychologists' work and the way in which they perceive the 'race', culture and ethnicity of pupils, parents and teachers, the social constructionist framework employed in the current study, also allowed me to remain flexible and open regarding my own and other educational psychologists' emotional experiences. Here, rather than feeling overcome or intimidated by expert knowledge, I had the opportunity to convey my own experiences and past knowledge and enter into a discourse with the educational psychologists, while "remaining aware of" the EPs "social and cultural context as well as the personal biases" the EPs themselves may have (Van Niekerk, 2005, p.69).

According to Hoffman (1991), all knowledge evolves in the realm of the 'common world' or the 'common dance' and only through on-going conversation with associates does the individual develop a sense of identity or an inner voice (p.5). Therefore, social constructionism exemplifies a collaborative and respectful framework, in which I aim to offer educational psychologists a context in which they could express and explore the emotions that they experienced regarding their work with those from diverse backgrounds.

\subsection{Purpose of Research}

According to Anderson and Goolishian (1988) the social constructionist perspective stipulates that there are no 'real' external entities that can be accurately mapped or apprehended. They argue that individuals are thereby forced to resign their cherished position as 'knowers' and their assumptions that there are 'facts' that they can come to know. These 'facts', along with other ideas and assumptions, are social constructions, artefacts of socially mediated discourse. Therefore, since the aim of this research is to initiate dialogue, 
awareness and understanding, it is hoped that me and the participants are able to engage equally as co-creators of a collective reality. In line with the social constructionist framework, I anticipate that by generating an opportunity of understanding and acceptance and by being deferential and genuinely interested as a co-participant in the meaning-generating process, the true emotions and behaviours of educational psychologists when working with those from diverse backgrounds can be explored. As such, the purpose of this study is to explore educational psychologists' experiences of working with pupils, parents or school staff from diverse backgrounds- those whose culture, 'race' and ethnicity are likely to differ from their own. In this study, the following research question is addressed:

What challenges do EPs face when providing services to CYP and/or families whose 'race', culture and ethnicity is different to their own?

\subsection{Context and Location of Current Study}

As the aim of the current study was to explore the experiences of educational psychologists working within a multiracial, multicultural and multi-ethnic context, educational psychologists from three London Boroughs were recruited. These boroughs were selected because the Simpson's Diversity Index (SDI) score of the 2011 Census identified the three boroughs as belonging to the top-thirty most diverse local authorities in London (2011 Census, Office for National Statistics). As reported by the Census Snapshot: Ethnic Diversity Indices (2011); the selected boroughs were very diverse in character and in all 3 boroughs, the population of individuals from ethnically diverse backgrounds ranged from $10 \%$ $40 \%$. According to each borough's Joint Strategic Needs Assessments from $2012,15 \%-85 \%$ of all pupils in primary and secondary schools were from a black or minority ethnic (BME) background. Within this population, 5\%-30\% of pupils were identified as Asian/British Asian, 5\%-15\% as African and Eastern European respectively. Figures for pupils from other minority groups, across the 3 boroughs, were not available. 
In England, data is also collected on pupils 'first language' "...thus offering a better insight on the diversity of pupils" (D'Angelo, Paniagua and Ozdemir, 2011) in schools. In England the national average of all primary school pupils who are known or believed to have English as an additional language is $17.5 \%$ and $12.9 \%$ of all secondary school pupils (Schools, Pupils and their Characteristics, Department for Education, 2013). A diverse range of pupil backgrounds was also evident from the fact that, in the boroughs selected for the current study; up to 150 languages- other than English- were identified as being spoken across all boroughs' primary and secondary schools.

With the work of educational psychologists mainly undertaken in schools and London's increasing diversity being reflected in its school population, the figures above indicate that "London as a whole now has an ethnic minority dominated...school system, akin to that of many large US cities..." (Hamnett, 2011). As such, it is important to understand the experiences of educational psychologists working within diverse boroughs such as those in the three participant boroughs to determine how they meet the needs of pupils, parents and/or teachers who are of a different racial, ethnic or cultural background to their own.

\subsection{Participants}

A purposeful sampling technique was used to recruit participants from across the 3 boroughs identified above. Purposeful sampling is described as selecting “...particular subjects [sic]... because they are believed to facilitate the expansion of the developing theory" (Bogdan and Biklen, 1998, p. 65). Since the purpose of the current study was to investigate educational psychologists' experiences of working with individuals whose 'race', culture and ethnicity was different to their own, twelve educational psychologists; eight female and four males, from the 3 London boroughs were invited to participate in the current research.

Participants ranged in age from 25 to 55 years old and, although a diverse group in terms of age, the majority of participants identified their ethnicity as White- 
English (8), followed by White- Irish (1), White- Welsh (1), British Asian (1) and Mixed- White and Black African (1) respectively.

\subsection{Ethical Considerations}

Ethical approval for the study was gained by the University of East London's University Research Ethics Committee. It should also be noted that the current research complies with the framework of the ethical principles outlined in the British Psychological Society's 'Ethics and Code of Conduct' (2006) and 'Principles for conducting Research with Human Participants' (1990).

Consent for participation was initially sought from the Principal Educational Psychologists (PEPs) or Service Managers of all three Local Authorities via email (Appendix IV). Once potential participants had been identified by the PEPs and their Service Managers, they were sent an email with further information about the study in question (Appendix V) outlining the overall aims of the study and ethical considerations relating to their participation in the study highlighted. Participants were given ample opportunities to contact me in the event of further information being required and were notified that they had a fortnight to conclude their wish to participate in the research study. This ensured that participants had sufficient knowledge, understanding and time to talk about their thoughts on the study with me and/or grant fully informed consent. Participants were then asked to give their written consent if they wished to participate in the study (Appendix $\mathrm{VI})$.

Although some research participants find it constructive to reflect on their life experiences (Birch and Miller, 2000), there remained a small probability, in the current study, of participants becoming anxious, agitated or overwhelmed when discussing instances of racism or multiculturalism. As such, participants were informed that they were not obligated to answer any questions they did not wish to and, that their interview would be stopped and discontinued if they chose not to proceed further. Finally, participants were provided with time to ask questions at the end of interviews and debriefed about the nature of the study (Appendix VII). 


\subsection{Data Collection}

In order to capture educational psychologists' experiences of working with those from diverse 'racial', cultural and ethnic backgrounds, data was collected in two phases, each of which reflected a distinct perspective from which to investigate the research question in the current study. This section outlines the basis for and implementation of the individual methods chosen for the current investigation. In the first phase, focus groups were used. The second phase consisted of individual semi-structured interviews with EPs from the focus groups.

\subsubsection{Phase One: Focus Groups}

Focus groups are "small structured groups with selected participants, normally led by a moderator. They are set up in order to explore specific topics and individual's views and experiences, through group interaction" (Litoselletti, 2003, p. 1). As well as it being an efficient way of collecting the multiple views of individuals' simultaneously, focus groups provide an opportunity for participants to engage in discussions and examine specific subjects collaboratively, with data emerging from the interaction between participants. Kitzinger (1994) argues that by accentuating communicative processes such as agreement, disagreement and group consensus, focus groups empower participants to become active agents in the analytic process. In addition, they can also help explore or generate hypotheses (Powell and Single, 1996) and develop questions or concepts for questionnaires and interview guides (Lankshear 1993).

With this is mind, the aim during this first phase of the current study was to collect a broad range of information about individual and group feelings, perceptions and attitudes related to 'race', culture and ethnicity.

\subsubsection{Focus Group Design}

In planning for the focus groups, a set of questions or "questioning route" (Krueger and Casey, 2000) was developed (Appendix VIII). This included a specific order in which questions were asked, starting with 'easy' questions 
relating to the participants' past thoughts and experiences in relation to race, culture and ethnicity to their current beliefs and practises and future aspirations. As suggested by Krueger and Casey (2000), questions were designed so they were open, sounded conversational and natural and flowed naturally from one question to another, moving from the general to the specific. Consequently, questions were designed to ensure that they were clear and easy to understand as well as being neutral. In addition, they were designed to gain a detailed and thorough understanding of the participants' responses, while at the same time, allowing me flexibility with which to further explore participants' specific responses to questions.

\subsubsection{Focus Group Procedure}

For ease of arrangement, the PEPs/Service Managers in each borough were asked to liaise with the potential participants in their service and identify a suitable day and time when all educational psychologists were expected to be at the office, for me to come in and run a focus group.

During the focus groups, participants were given a brief introduction to the research area, including an overview of the local school population. Key terms: 'race', culture, ethnicity and black, minority ethnic (BME) were also defined (Appendix IX) to ensure transparency and consistency in responses as well as a way of prompting discussion around complex and potentially sensitive issues. Participants were then encouraged to consider their experiences of working with pupils, parents and school staff from racially, ethnically and culturally diverse backgrounds.

Three separate focus groups were carried out as part of the current study and twelve educational psychologists, ranging in age and experience, participated in the focus groups across the three boroughs (Table 1). 
Table 1: Focus Group Characteristics

\begin{tabular}{|c|c|c|c|c|c|c|}
\hline \multirow{2}{*}{ Borough } & Number of & \multicolumn{3}{|c|}{ Sex } & \multicolumn{4}{c|}{ Age Range } \\
\cline { 3 - 8 } & participants & Male & Female & $25-35$ & $36-45$ & $46-55$ \\
\hline A & 3 & 1 & 2 & 1 & 1 & 1 \\
\hline B & 6 & 0 & 6 & 2 & 2 & 2 \\
\hline C & 3 & 3 & 0 & 2 & 1 & 0 \\
\hline
\end{tabular}

All focus groups lasted between 45-70 minutes and took place in quiet and welllit rooms within the Local Authority, which were readily accessible to the participants.

Participants' responses were recorded using an Olympus DM-5 Digital Voice Recorder and reflective notes made by me before and after each focus group.

\subsubsection{Phase Two: Semi-Structured Interviews}

According to Smith, Flowers and Larkin (2009), a semi-structured interview can be referred to as "a conversation with a purpose" (p.57) in order to gain insight into the experience, feelings, values or opinions of a participant (Esterberg, 2002). Methods vary in the extent to which respondents are directed to discuss given topics and to which they are given freedom to express and explore their replies (Coolican, 2004). In semi-structured interviews, a guide is often used, with questions and topics that must be covered by the researcher. Here, although I had some discretion about the order in which questions were asked, the questions were standardised and probes were provided to ensure that I covered the correct material (Harrell and Bradley, 2009).

\subsubsection{Semi-Structured Interviews Design}

Since the aim of the semi-structured interviews was to allow the EPs who had participated in the Focus Groups to talk about their beliefs and opinions at length and develop their narratives about 'race', culture and ethnicity, the semistructured interviews enabled flexibility, yielded rich data and also allowed me to dissect and further explore any substantial and meaningful topics or issues that were discussed or conveyed during the focus groups (Smith, Flowers and Larkin, 
2009). The conversational nature of semi-structured interviews (Harrell and Bradley, 2009) allowed me to delve deeply into the topic of educational psychologists working with those from diverse backgrounds.

\subsubsection{Semi-Structured Interviews Procedure}

Individual face-to-face semi-structured interviews were conducted as part of the current study. Participants were contacted via email and agreed to meet at a mutually convenient time within the Educational Psychology Service of their Local Authority. When meeting with each participant, I thought it imperative to remind each participant about the aims of the study, the structure of the semistructured interview and their right to withdraw at any point up until their responses from the semi-structured interview had been transcribed (i.e. Appendix V). All interviews lasted between 45- 75 minutes approximately.

Of the original twelve participants, two participants (British Asian and MixedWhite and Black African) withdrew from the study during this stage. As a result of this, the semi-structured interviews were conducted with ten, white, British Educational Psychologists only.

\subsection{Trustworthiness}

Since the concepts of reliability and validity do not apply in the same way in qualitative research as they do in quantitative research, the rigor and trustworthiness of qualitative research has frequently been questioned by critics of qualitative research. Despite this, advocates of qualitative research have been instrumental in demonstrating how qualitative researchers can integrate processes that address this issue (e.g. Silverman, 2001; Pitts, 1994). In addition, they have espoused using different terminology to set themselves apart from the positivist paradigm. For example, Guba (1981) suggests that qualitative researchers should consider four criteria to determine the trustworthiness of a study; credibility, transferability, dependability and confirmability. By addressing similar issues, Guba's constructs correlate to the principles of internal validity, external validity/generalisability, reliability and objectivity as employed by the 
quantitative researcher (Shenton, 2004). Addressing Guba's concepts of credibility, transferability, dependability and confirmability, Shenton (2004) presents a range of strategies that can adopted by qualitative researchers to ensure trustworthiness in their study (Table 2).

Table 2: Provisions that may be made by a qualitative researcher wishing to address Guba's four criteria for trustworthiness.

\begin{tabular}{|c|c|}
\hline $\begin{array}{l}\text { Quality } \\
\text { Criterion }\end{array}$ & Possible Provision made by Researcher \\
\hline Credibility & $\begin{array}{l}\text { - Adoption of appropriate, well recognised research methods } \\
\text { - Development of early familiarity with culture of participating organisations } \\
\text { - Random sampling of individuals serving as informants } \\
\text { - Triangulation via use of different methods, different types of informants and } \\
\text { different sites } \\
\text { - Tactics to help ensure honesty in informants } \\
\text { - Iterative questioning in data collection dialogues } \\
\text { - Negative case analysis } \\
\text { - Debriefing sessions between researcher and superiors } \\
\text { - Peer scrutiny of project } \\
\text { - Use of "reflective commentary" } \\
\text { - Description of background, qualifications and experience of the researcher } \\
\text { - Member checks of data collected and interpretations/theories formed } \\
\text { - Thick description of phenomenon under scrutiny } \\
\text { - Examination of previous research to frame findings }\end{array}$ \\
\hline Transferability & $\begin{array}{l}\text { - Provision of background data to establish context of study and detailed } \\
\text { description of phenomenon in question to allow comparisons to be made }\end{array}$ \\
\hline Dependability & $\begin{array}{l}\text { - Employment of "overlapping methods" } \\
\text { - In-depth methodological description to allow study to be repeated }\end{array}$ \\
\hline Comfirmability & $\begin{array}{l}\text { - Triangulation to reduce effect of investigator bias } \\
\text { - Admission of researcher's beliefs and assumptions } \\
\text { - Recognition of shortcomings in study's methods and their potential effects } \\
\text { - In-depth methodological description to allow integrity of research results to be } \\
\text { scrutinised } \\
\text { - Use of diagrams to demonstrate "audit trail" }\end{array}$ \\
\hline
\end{tabular}

(Shenton, 2004, p.73)

In the current study, the framework suggested by Shenton (2004) in addressing Guba's four criteria to ensure rigor and trustworthiness in qualitative research were adopted.

In addressing credibility, I ensured that a true picture of the phenomenon under scrutiny was being presented to the participants. This was done through creating familiarity with the culture within which the participants worked before the commencement of the initial data collection dialogue. This took place via 
consultation of appropriate documents and preliminary visits to the organisations themselves. Triangulation was also used to ensure credibility of data through the use of a number of participants within each organisation. This triangulation via data sources ensured that the views and experiences of each individual could be verified against others, resulting in a comprehensive representation of the beliefs, needs and attitudes of those participating in the study based on the contributions from several individuals. Site triangulation was also achieved through the participation of individuals from three local boroughs in order to decrease the outcome of specific indigenous aspects related to one borough. According to Shenton (2004), selecting a range of participants in diverse organisations "...may be employed to provide the diversity that underpins the concept of "circling reality" (p. 66) whereby one obtains "... a variety of perspectives in order to get a better, more stable view of 'reality' based on a wide spectrum of observations from a wide base of points in time-space" (Dervin, 1983, p.7). In order to allow transferability, I have provided necessary information of the context within which the current study took place. Here the aim is for the reader to gain a comprehensive insight from the accumulation of findings from studies staged in different settings and to make transferability inferences (Shenton, 2004).

Although ensuring dependability can be problematic in qualitative work (Marshall and Rossman, 1999) due to the "published descriptions" being "static and frozen in the 'ethnographic present"' (Florio-Ruane, 1991, p. 5), Lincoln and Guba (1985) emphasise the strong association between credibility and dependability and state that practicing credibility helps ensure dependability. One way of achieving this, includes the use of "overlapping methods" (Shenton, 2004, p.71) such as focus groups and individual interviews as employed in the current study. Attempts at ensuring dependability in the current study were also made by reporting in depth the processes of the study, enabling future researchers "...to repeat the work, if not necessarily to gain the same results" (Shenton, 2004, p. 71). Moreover, the continued use of a "critical friend" (Banister, Burman, Parker, Taylor and Tindall 1994) was also considered to ensure consistency in data analysis and to aid in identifying potential themes within the data corpus. It was felt that this rigorous and meticulous method would ensure transparency in the data analysis, leaving it open to the scrutiny of the reader. 
Finally, I attempted to achieve confirmability by undertaking steps to ensure that results in the current study were the direct outcome of the participants' thoughts, ideas and experiences rather than her own philosophies and assumptions. One way of ensuring this was by recording the participants' responses within the focus groups and semi-structured interviews. After transcribing each recording, I read through the scripts while listening to the audio recordings to ensure accuracy of data and to amend transcripts, if required. It is important to note that each script was transcribed as verbatim as possible to produce an "orthographic" record (Poland, 2002) of the participants' responses (Appendix X). A dual-analysis of the transcripts was also conducted, once all transcripts had been transcribed, to ensure consistency and further accuracy of participants' responses (e.g. a small number of transcriptions were checked by an independent researcher). All transcripts were then prepared for qualitative analysis.

\subsection{Data Analysis}

Glesne and Peskin (1992) state that in order to analyse data from research, one must organise what has been seen, heard and read so that sense can be made of what is learned. However, as the analysis of data in qualitative research “...takes place throughout the entire research process, a study is shaped and reshaped as a study proceeds, and data is gradually transformed into findings" (Watt, 2007, p.98). However, as indicated by Miles and Huberman (1994), "...each qualitative study is unique, the analytical approach used will be unique" (p. 433).

The distinctive feature of qualitative data- and its analysis- is a focus on text. Here, qualitative analysts may view text as a way to understand what research participants "really" thought, felt or did in some situation or at some point in time. Alternatively, they may adopt a hermeneutic perspective on texts; a perspective that views a text as an interpretation that can never be judged true or false (Miles, Huberman, Saldana, 2014, p. 321), thus indicating that "the text is only one possible interpretation among many" (Patton 2002, p. 114). As qualitative data analysts seek to describe their textual data in ways that capture the setting or people who produced the text on their own terms rather than in terms of 
predefined measures and hypotheses, qualitative data analysis tends to be inductive with an emic, rather than etic focus, where important categories in the data, as well as patterns and relationships, are identified.

When deliberating over the suitability of a number of analyses for the current study, the aim was to discover an approach which would allow the procurement of a thorough understanding of the attitudes, thoughts and opinions of the professionals involved in the research. As the data corpus for the current study comprised of two data sets; focus groups and semi-structured interviews, two differing qualitative research methods were considered; Grounded Theory (Glaser and Strauss, 1967) and Thematic Analysis (Merton, 1975; Braun and Clarke, 2006). At the beginning of the research process, I contemplated employing critical realism as an epistemological approach and grounded theory as a method of analysis in the current study as it was believed that these would permit flexibility in integrating findings from the EPs across the three boroughs. However, as the concepts of 'race', ethnicity and culture do not occur in a social vacuum (e.g. Machery and Faucher, 2005), it was decided that social constructionism would be deployed as the epistemological approach and Thematic Analysis as the qualitative approach to analyse data.

\subsubsection{Thematic Analysis}

The method of analysis selected for the current study was Thematic Analysis (Braun and Clarke, 2006) owing to its breadth of analysis and its capacity in allowing the data from the various participants to be conceptualised and compared. Specifically, it was felt that this model would provide a framework for an exploration of the consistencies, incongruities and areas for systemic development in the experiences of educational psychologists working in culturally, racially and ethnically diverse schools.

As illustrated in Braun and Clarke's (2006) paper; thematic analysis offers epistemological flexibility with due regard to rigour. For the purposes of clarity, the use of Thematic Analysis in the current study has been defined using the key terms identified by Braun and Clarke (2006). These terms contextualise analysis 
of the data with due regard to the role of researcher as an active participant in the interpretation process.

\subsubsection{Thematic Analysis and Themes}

Thematic Analysis "... is a method for identifying, analysing, and reporting patterns (themes) within data. It minimally organises and describes... data set in (rich) detail" (Braun and Clarke, 2006, p. 6). However, it also often goes further than this, and interprets various aspects of the research topic (Boyatzis, 1998). A theme, according to Braun and Clarke (2006), “...captures something important about the data in relation to the research question and represents some level of patterned response or meaning within the data set" (p. 10). Braun and Clarke (2006) also define themes both in terms of their prevalence in terms of space within each data item and prevalence across the entire data set and state that although there may be a number of occurrences of the theme across the data set, more occurrences do not mean the theme itself is more crucial. They state that as themes are not solely defined by prevalence, they should not be based on quantifiable measures (as in content analysis) and should be defined in terms of whether they capture something important in relation to the overall research question(s) (Braun and Clarke, 2006). Here, prevalence of a theme can be determined in a number of ways from counting the frequency of the data item across the entire data set or each individual occurrence of the theme across the entire data to the number of speakers who articulated the theme. It is important to note that as there is no "...right or wrong method for determining prevalence" (Braun and Clarke, 2006, p. 11) of themes, the researcher consequently becomes an active participant in generating themes within the reflective process of data analysis.

\subsubsection{Inductive versus Theoretical Analysis}

Themes (or patterns) within data in thematic analysis can be identified in one of two primary ways; using either an inductive or theoretical approach. The inductive approach requires themes to be identified and be strongly linked to the data. Here, the themes identified may indicate little association to the specific 
questions that were asked of the participants and would not be driven by the researcher's theoretical interest in the area or topic (Braun and Clarke, 2006). During inductive analysis, the researcher would attempt to code “...the data without trying to fit it into a pre-existing coding frame, or the researcher's analytic preconceptions" (p. 12). In contrast, the theoretical approach necessitates themes to be identified by the researcher's theoretical basis or research questions. Theoretical analysis is therefore analyst rather than data driven. The theoretical approach has been criticised because detailed accounts of the data are not produced with the focus, instead, being given to a particular area within the data set. Consequently, some themes may not be identified and be overlooked. When considering educational psychologists' direct practice towards those from diverse backgrounds, I considered it important to view educational psychologists' present practices and beliefs without any preconceptions. As a result of this, inductive analysis was used within the current research to gain an insight into the views, experiences and practices of educational psychologists working with those with a different racial, cultural or ethnic background to their own.

\subsubsection{Semantic and Latent Themes}

Themes, within thematic analysis, can be identified at a semantic or a latent level (Boyatzis, 1998). At a semantic level, the themes are identified within the explicit or surface meanings of the data and the analyst is not looking for anything beyond what a participant has said or what has been written. Here, the analysis involves a move from description to interpretation (Patton, 1990), often in relation to previous literature (Braun and Clarke, 2006). According to Braun and Clarke (2006), themes identified at the semantic level tends to conform to a constructionist paradigm (e.g., Burr, 1995) “....where broader assumptions, structures and/or meanings are theorised as underpinning what is actually articulated in the data" (p. 13).

Although the epistemology in the current research allows reflection on wider

systemic influences, the purpose of the research at this stage within the research process is exploratory in nature. Therefore, given that the method of data 
collection involves focus groups and semi-structured interviews, it would be questionable to over-analyse the data corpus. Therefore, data was analysed at the semantic level in the current study. However, a flexible approach was adopted to identify other themes which may arise external to the theoretical approach, particularly as this research study was adopting an exploratory methodology.

\subsubsection{Essentialist/Realist versus Constructionist Thematic}

\section{Analysis}

Braun and Clarke (2013) state that although some researchers (e.g. Joffe, 2011; Guest, MacQueen and Namey, 2012) view thematic analysis as a phenomenological approach, owing to its theoretical flexibility, thematic analysis is "...just an analytic method, rather than a methodology, which most other qualitative approaches are..." (p. 120). According to Braun and Clarke (2006), thematic analysis is "...theoretically flexible because the search for, and examination of, patterns across language does not require adherence to any particular theory of language, or explanatory meaning framework for human beings, experiences or practices" (p. 120). This, they maintain, suggests that thematic analysis “...can be applied within a range of theoretical frameworks, from essentialist to constructionist..." (p.120). For example, as a researcher's epistemological position governs how research data is analysed to theorise meaning, from an essentialist (or realist) perspective, a researcher may infer incentives, experiences and meanings in a conspicuous manner because a simple, largely unidirectional relationship is assumed between meaning and experience and language (Braun and Clarke, 2006). However, from a constructionist viewpoint, meanings and interpretation of experiences are socially produced and reproduced, rather than inherent within individuals (Burr, 1995). Here, thematic analysis “...conducted within a constructionist framework cannot and does not seek to focus on motivation or individual psychologies, but instead seeks to theorise the socio-cultural contexts, and structural conditions, that enable the individual accounts that are provided" (Braun and Clarke, 2006, p. 14). 


\subsubsection{Conducting Thematic Analysis}

Having first been named as an approach by Merton (1975), several different versions of thematic analysis have since been proposed within psychology, from Aronson, 1994, Attride-Stirling, 2001, Boyatzis, 1998, Joffe and Yardley, 2004, Tuckett, 2005 to Braun and Clarke (2006). According to Braun and Clarke (2006), although thematic analysis is extensively deployed in qualitative research, there is little or "...no clear agreement about what thematic analysis is and how you go about doing it" (p. 6). As a response to its poor demarcation, Braun and Clarke (2006) identified and defined six phases for systematically and accountably conducting a thematic analysis (Appendix XI).

\subsubsection{Thematic Analysis in the Current Study}

As the aim of the current study is to acquire a detailed account of the extensive experiences of educational psychologists in the UK when working with those from diverse backgrounds, thematic analysis fits in well with the research question as it can augment existing research by means of facilitating an in-depth study and producing a rich description of themes rather than an in-depth account of a particular theme. In addition, undertaking a comprehensive thematic analysis as an initial measure towards developing a research based understanding of any potential differences in participants' experiences enabled exploration of themes across settings, facilitating broader, more systemic observations to be drawn. As such, thematic analysis is well suited for exploratory research, as is the aim of the current study.

As thematic analysis does not necessitate the researcher to maintain an objective distance from the participants, tacit knowledge can, therefore, legitimately be linked with the participants' thinking to help generate a more local and contextual theory to answer the focus questions. However, there must be an acknowledgement by the researcher that the data is only a 'snapshot' of what is happening at that particular time (Mehmetoglu and Altinay, 2006). Care must be taken that the snapshot is not interpreted as the truth but forms more of a conceptual insight. 
Table 3 provides a summary of thematic analysis based on the six phases suggested by Braun and Clarke (2006). In light of the data from the three focus groups, these phases were modified for the current study. 


\section{Table 3: Phases of Thematic Analysis in the Current Study}

I was actively involved in all areas of data collection (focus groups) and the subsequent transcriptions, it was at these points that initial ideas for potential themes from the data first emerged. Here, the transcripts were read twice; the first time while playing the recording of the interviews to ensure the meaning of the words was accurately understood and noted. During the second reading, notes were taken that were to inform coding development and all relevant and interesting data extracts (i.e. textual quotes) were identified through highlighting text and the use of coloured 'post it' notes.

All three focus groups were separately analysed and all data extracts were grouped according to interview questions. These were then examined to identify initial codes. Once initial codes had been identified for each of the interview questions, they were organised into meaningful groupings (i.e. all data extracts were collated and those with similar codes were put together). Codes were simple descriptions of the data and were therefore different to themes in that they had narrower scope. The coding process was carried out manually and aided by the use of highlighting text and 'post-it' notes which allowed me to manually group data extracts. Dual researcher coding analysis was employed to ensure that coded groupings were reflective of the data extracts and to ensure that all the data extracts under each code reflected the same type of information. Through this dual process, codes were edited, amalgamated and dismissed, until codes were agreed to be reflective of the overall dataset. However, when codes reflected data extracts from only one transcription and deemed inappropriate to amalgamate with other codes, they were retained in order to reflect the diversity of experiences.

With a focus on searching for developed themes during the third phase of thematic analysis, the initial codes generated during the second phase of the study, were arranged into overarching or 'developed' themes. Developed themes were identified at the manifest level; directly observable in the information (see Boyatzis, 1998) and used to describe participants' responses by putting like with like (Morse and Field, 1995) across each focus group data set. This was so that any similarities and differences across participants' responses could be identified as well as ensuring that the data did not support the author's own pre-conceptions. In so doing, this ensured the tenets of social constructivism to be integral to the process of inductive/data analysis as it allowed the themes to develop directly from the data. At this stage, all coded data extracts were used. The majority of the codes became themes and no data was excluded at this point. During supervision with an experienced researcher, developed themes were edited, amalgamated and/or rejected until it was agreed that the themes represented an accurate reflection of the codes and the data extracts. This was to ensure inter-coder reliability.

During this phase developed themes from the previous phase were refined and the focus group data further scrutinised to ensure that there was enough information to support any main themes that had been identified. In addition, when miscellaneous data was found amongst a theme, themes were broken down further. Internal homogeneity and external homogeneity (see Patton, 1990) was considered respectively meaning that data under each theme was coherent and that there were distinct differences between the themes. Data extracts under each theme were then re-read to ensure that a coherent pattern had been formed. Main themes were considered in terms of the whole dataset and adjusted accordingly to represent the meanings of the entire dataset (see appendix XII for a summary of the initial codes, developed themes and main themes identified for focus groups 1,2 and 3). 

D 舟 政 themes were recognised. A thematic map for each of the three focus groups was $\sum \stackrel{\widetilde{N}}{5}$ then developed (Appendix XIII). An ultimate thematic map that combined all the themes from each of the focus groups into final themes and any ensuing sub-

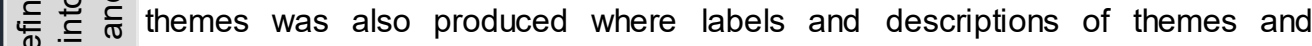

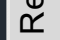
subthemes were edited to ensure clarity and transparency.

The next chapter represents the final analysis of the thematic analyses of the data

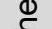
corpus. The most vivid examples of data extracts from the three focus groups were included alongside the themes and subthemes. In addition, the individual semistructured interviews were also examined for excerpts that supported the subthemes and included as necessary. Each theme was considered to 'tell a story' 0 and was analysed and discussed separately, relating back to the research question and literature. 


\section{Chapter 4: Findings}

This chapter reports on the challenges of CYP and their families' 'race', culture and ethnicity on the practice of white, British Educational Psychologists as identified by the thematic analysis. Figure 1 provides an overall thematic map with final themes and sub-themes, identified from the three focus groups and further reinforced by the ten semi-structured interviews, to illustrate these challenges.

Figure 1: Challenges encountered by white, British Educational Psychologists when working with BME children, young people and their families. 


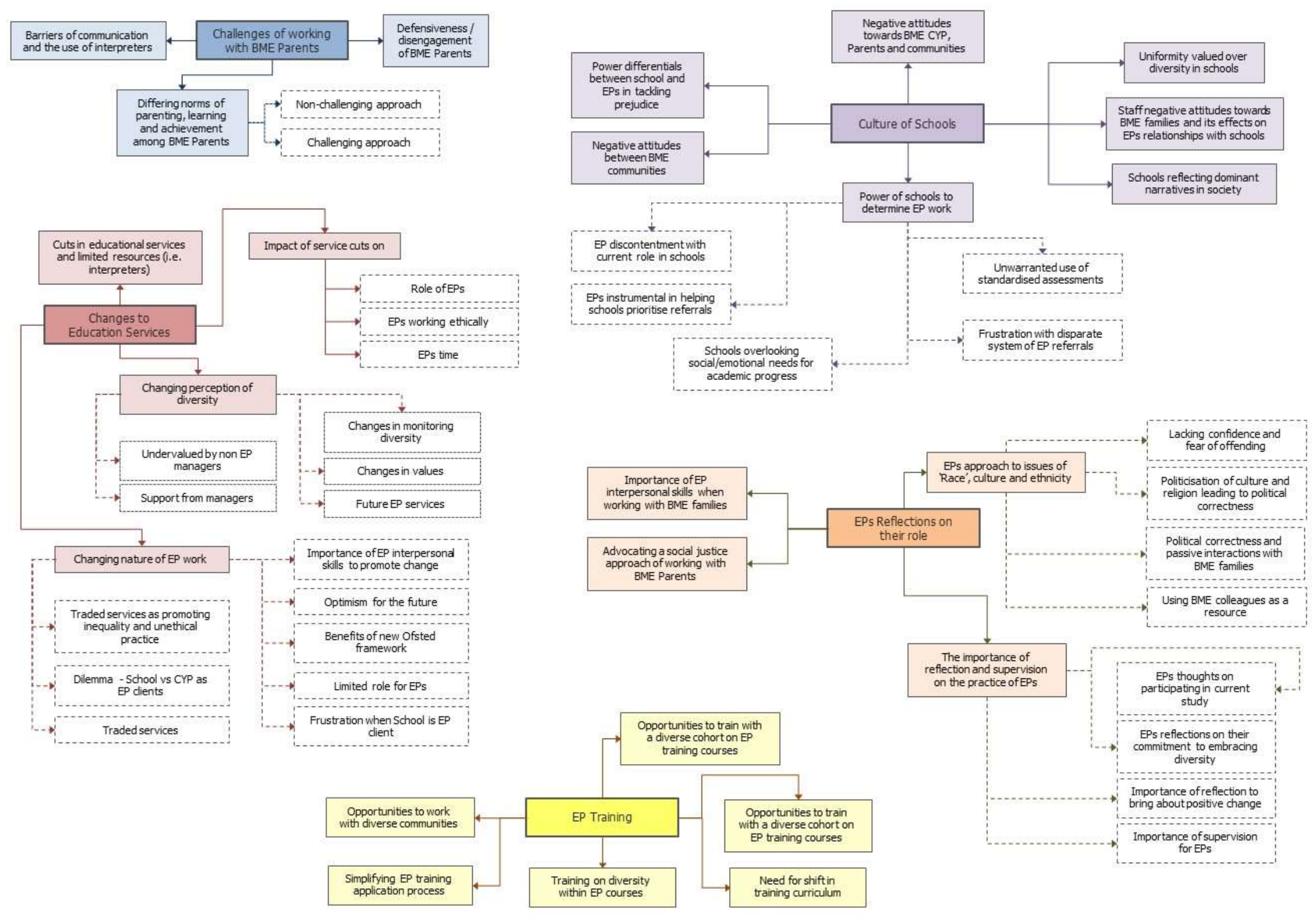


Details of the final themes and sub-themes presented in Figure 1 are outlined below in detail (see Table 4 for an overview). Here, it must be noted that the subthemes do not rank the significance of any one theme; instead sub-themes are used to illustrate the themes that materialised from the focus group data. The themes and sub-themes that developed from the focus groups are supported by excerpts from the 1:1 semi-structured interviews, to ensure that the interpretation of the data was directly linked to the words of the participants. Excerpts to support or illustrate main themes and their resulting sub-themes have been presented verbatim, without grammatical changes being made to ensure authenticity of participants' responses.

Table 4: A summary of the themes and sub-themes that developed from the three focus groups on the challenges encountered by white, British Educational Psychologists when working with BME children, young people and their families across three London boroughs.

\begin{tabular}{|c|c|}
\hline Final Themes & Sub-Themes \\
\hline 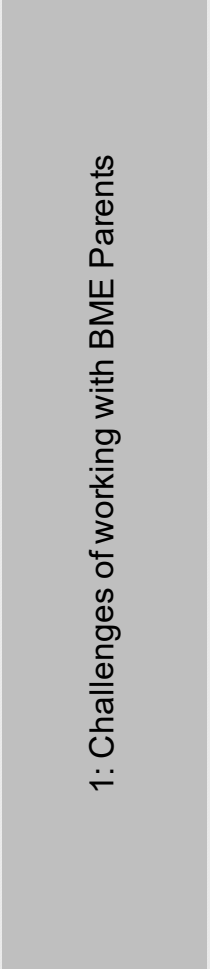 & $\begin{array}{l}\text { Sub-Theme 1.1: Differing Norms of Parenting, Learning and Achievement } \\
\text { among BME Parents } \\
\text { - Non-Challenging Approach } \\
\text { - Challenging Approach } \\
\text { Sub-Theme 1.2: Defensiveness/Disengagement of BME Parents } \\
\text { - BME Parents Passivity in Accessing Services } \\
\text { - BME Parents Lacking Resources and Skills to Advocate for their CYP } \\
\text { - Schools Negative Attitudes towards BME CYP and/or Families } \\
\text { - Contentious Attitudes of School Staff } \\
\text { - BME Parents with Underlying SEN } \\
\text { - Cultural Factors Leading to Disengagement } \\
\text { - BME Parents Disengaging when EPs from non-BME Background } \\
\text { Sub-Theme 1.3: Barriers of Communication } \\
\text { - BME Parents Lack of Spoken English } \\
\text { - Lack of Shared Language leading to Confusion } \\
\text { - Importance of Communicating with Mothers } \\
\text { - Parents Limited English Language Skills and Access to Groups } \\
\text { - Language Barrier to the Assessment Process } \\
\text { - Simplifying EP Reports } \\
\text { - Difficulties when using Labels } \\
\text { - Challenges with use of Interpreters }\end{array}$ \\
\hline
\end{tabular}


Sub-Theme 2.1: Negative Attitudes towards BME CYP, Parents and Communities

Sub-Theme 2.2: Schools Reflecting Dominant Narratives in Society

Sub-Theme 2.3: Negative Attitudes between BME Communities

Sub-Theme 2.4: Uniformity valued over Diversity in Schools

Sub-Theme 2.5: Staff Negative Stereotypes and its Effects on EPs Relationship with Schools

Sub-Theme 2.6: Power Differentials between School and EPs in Tackling Stereotypes

Sub-Theme 2.7: Power of Schools to Determine EP Work

- EP Discontentment with current Role in Schools

- Frustration with Disparate System of EP Referrals

- Unwarranted Use of Standardised Assessments

- EPs Instrumental in helping Schools Prioritise Referrals

Sub-Theme 3.1: Cuts in Educational Services and Limited Resources

Sub-Theme 3.2: Impact of Service Cuts on EPs

- Impact of Service Cuts on the Role of the EP

- Impact of Service Cuts on EPs working Ethically

- Impact of Service Cuts on EPs Time

Sub-Theme 3.3: Changing Perception of Diversity

- Changes in Monitoring Diversity

- Change in LA Values

Sub-Theme 3.4: Changing nature of EP Work

- Traded Services

- Dilemma- School vs CYP as EP Clients

Sub-Theme 4.1: Opportunities to work with diverse communities

일 Sub-Theme 4.2: Opportunities to train with a diverse cohort on EP training courses

Sub-Theme 4.3: Promoting Diversity within EP Training Staff and Services

Sub-Theme 4.4: Training on diversity within EP courses

Sub-Theme 4.5: Need for shift in training curriculum 


\begin{tabular}{|c|c|}
\hline Final Themes & Sub-Themes \\
\hline 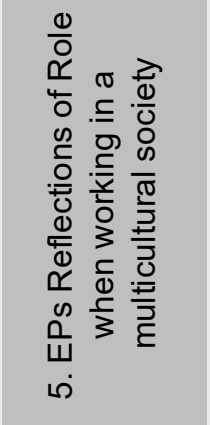 & $\begin{array}{l}\text { Sub-Theme 5.1: EPs Approach to Issues around 'Race', Culture and Ethnicity } \\
\text { - Lacking Confidence and Fear of Offending } \\
\text { - Politicisation of Culture and Religion leading to Political Correctness } \\
\text { - Using BME Colleagues as a Resource } \\
\text { Sub-Theme 5.2: Importance of EPs Inter-Personal Skills when working with } \\
\text { BME Families } \\
\text { Sub-Theme 5.3: Advocating a Social Justice Approach of working with BME } \\
\text { Parents }\end{array}$ \\
\hline
\end{tabular}




\subsection{Final Theme 1 - Challenges of working with BME Parents}

As a main theme, challenges of working with BME parents resulted in three subthemes ranging from cultural differences of parenting, learning and achievement, defensiveness of BME parents as well as barriers of communication and using interpreters.

\section{Sub-Theme 1.1 Differing Norms of Parenting, Learning and Achievement among BME Parents}

EPs in the focus groups discussed their reactions to a number of areas where they felt uncomfortable interacting with BME parents. One area reported was that of conducting consultations with BME parents and it becoming apparent that families from certain BME backgrounds had rigid norms of parenting and what constituted learning. These were either at odds with their own and/or highlighted the EP's lack of knowledge related to certain cultural practices.

Similarly, EPs also noted coming across parents who felt that children playing with toys was frivolous and unrelated to learning. Parents from BME backgrounds who stressed the importance of their offspring attaining good grades were also a cause for concern.

There were a number of ways EPs dealt with these from adopting a nonchallenging to challenging approach with parents.

\section{- Non-Challenging Approach}

PK: $\quad$...So, for this particular little boy, who had Downs Syndrome where she [Portage Home Visitor] was going round into the home and trying to get the mother to play more, to play more str-, in a more structured way, she said it was really, really hard because it just wasn't part of their... it was a lovely mother. She was a really lovely lady but just sit down and play with her son, was something that she just, she didn't... and she had three or four other children, they were older, this was the last one and erm, she said it was part of the Somali culture and I hadn't really heard that before but, I remember thinking "Oh, that's, that's interesting!" (95-101). 
EPs justified their passivity by stating that directly challenging cultural practices and/or established norms may result in BME parents' disengagement and/or complete withdrawal from services:

RHA: ...At the end of the day, there's no point in challenging what's important to them because they're just going to disengage with you... (831-832).

PK: We could be faffing around about upsetting someone and at the end of the day, there could be a child suffering... (352-353) ... not physically suffering but, mentally, being tormented about a situation because all the professionals who were involved with that child are thinking: We don't want to insult someone who's a Muslim (355-357).

\section{- Challenging Approach}

In some instances, EPs intervened as an attempt to make parents see a situation from their perspective:

PK ...and, sometimes I explain to them why we don't smack our children, why, you know, it's not illegal but, you know, there are reasons why smacking isn't... isn't accepted, erm, you to help people understand (882-884).

One EP felt that whilst he respected the views of the parent, he felt they were at odds with his own views. Here, the EP did not challenge the views of the parent directly but made subtle recommendations based on psychological theories and models, believing that providing the parent with this information would allow her to shift her perspective on her child's learning needs:

EN: I also mentioned about the benefits of, I suppose, I believe and there's studies about, erm, things about learning, rote's only one aspect about what makes somebody successful- you know, play teaches things like resilience, determination, which- those kind of things are equally important, those will be things that make people successful. So I expressed that, I don't think- well no it didn't go in [laughs]! Yeah. So, I tried to put evidence to it and I tried to put it in a sensitive way (211-223).

Another EP elaborated on this and reflected on the dissonance she felt when working with parents from a specific cultural background and the importance they placed on deploying private tutors and/or rote-learning to help their child do well at school, often resulting in a disaffected and tired child: 
RHA: And so... I don't know... again, this is my naivety to what goes on in other countries and cultures, but that just does seem to be a pattern when there seems to be a lot focus on achievement in some cultures and a lot of like just drilling these children sometimes. And that doesn't sit easy with me, but then again, it's about just being open to and just discussing the child's wellbeing with that parent. And ultimately, it's their decision I suppose if they want to have four hours of tutoring in the evening for that child. You can then say, "This is probably going to be the implications of giving that, of doing that to the child" (131-140).

As EPs gained experience of working with BME parents, they appeared to realise that though there were views for learning and achievement among BME parents, there were also differences within some cultural groups. This highlighted that fact that cultural sensitivity should not lead to cultural stereotypes.

One EP reported coming across the case of a family with a child with learning difficulties and being perplexed by the parent's reluctance to have her child attend a special school due to the cultural stigmas attached to this. In a 1:1 interview, she stated:

PK: Her parents had very strong views about special schools; they didn't want her... and the mother did say something to me like 'but we won't be able to marry her'. No one was going to want to marry a girl who's been to special school and that did kind of make me think about arranged marriages and maybe skew my views a bit but since then I have very few families that say that. I think you start to realise that not all families are like that; just because you're Muslim doesn't mean you're going to marry off your girls and ignore the fact they have learning difficulties (565-577).

\section{Sub-Theme 1.2 Defensiveness/Disengagement of BME Parents}

This sub-theme comprised of seven components, i.e. BME Parents Passivity in Accessing Services, BME Parents Lacking Resources and Skills to Advocate for their CYP, Schools' Negative Attitudes towards BME CYP and/or Families, Contentious Attitudes of School Staff, BME Parents with Underlying SEN, Cultural Factors Leading to Disengagement and BME Parents Disengaging when EPs are from non-BME Backgrounds. These are discussed in detail, below.

\section{- BME Parents Passivity in Accessing Services}


EPs reported experiencing frustration in working with submissive parents from some BME backgrounds who initially lacked knowledge about services available to them and, who, despite being alerted to the availability of services and how these could result in having positive outcomes for their child, still remained inactive in accessing those services.

RHA: and then you've got a Soma- which I did last year-a Somali family... (256258)...the son was in reception class. He has such severe autism, and it was diagnosed. He has such severe learning difficulties, sensory difficulties. He could not cope with simply being in the school environment no matter what interventions the school put in place: one-to-one, in a quiet room. He was just... he could not access that environment. And you know what? If that would have been the white middleclass family, they have an awareness of... I don't know whether it is a cultural thing, but I just felt really frustrated at that point because the family were just so grateful having recently arrived... well, not recently... within the past few years. They were just grateful. They kind of... for all the help they were getting. And I was like, "No, stomp your feet. Your child's been out of school for a year now because the school haven't been able to support you (262270)...

\section{- BME Parents Lacking Resources and Skills to Advocate for their CYP}

EPs reported that apathy from parents or their disengagement from services was less about the ethnic background they belonged to and more about how wealthy they were. Here, an EP noted that parents who could afford more and had access to resources were the ones who would insist that schools, professionals and LAs met the needs of their child.

CT: ... we're all very well aware of the fact that in [borough], there's a very big problem around those that have... a considerable wealth and the, erm, unequal distribution and I think we're talking and thinking about our young people who come in from other boroughs who are not even entitled to the same range of services that the other children are- owing to those different factors (139-145).

I think it's less about ethnic background and far more about wealth. It doesn't matter what ethnic background you're from; if you can afford Z [Public School] or you can afford to buy in resources then that's different to where you can't (421-423).

However, another EP reported that parents from BME communities lacked social and cultural skills to advocate on behalf of their child: 
CD: ...parents- some not all- from some ethnic groups don't have the language skills, the social, the cultural capital, the social skills, the resources to engage with schools on behalf of their children. In many ways, it is not dissimilar to the whole issue about social class and middle-class institutions- if you don't fit that model it is much harder and that is true for many minority ethnic groups (41-46).

One EP reflected on her experiences of working in several local authorities in London and her coming face-to-face with the various issues as an EP.

BFP: you know, issues are different per decade. There is a buzz around some issues that last for a particular time and then up comes another one. I think initially there was a lot of concern about the success of the Afro-Caribbean population when I started working, and very recently that's changed to a big concern with working class boys. So I think, over the years, issues are tackled, and I think that that's largely been a very positive thing. There's been a lot of thinking, a lot of talking, a lot of reflection, and I think that leads to good outcomes for children in general (92-101).

She also mentioned BME parents being disadvantaged and disempowered in terms of a lack of access to resources and schools making things worse by not allowing parents to have a voice or to take ownership of decisions related to their CYP's education.

BFP: I think a thought that I always have is about socio-economic factors and experiences of powerlessness in people from ethnic minority backgrounds. So many times l'll come across an attitude that parents are uncomfortable about their child being assessed because they feel you might be... you are going to take away the child. And I just wonder if we're doing enough work in schools about parental empowerment. And that schools, I think, keep rather a distance and have a rather paternalistic role in the lives of the children (101-117).

\section{- Schools Negative Attitudes towards BME CYP and/or Families}

EPs in the Focus Groups felt surprised that during consultations school staff did not always acknowledge the cultural and/or linguistic diversity and/or experiences of CYP from BME communities:

MJ: I am quite shocked how it feels like lot of the [borough] schools are quite behind in terms of understanding all these issues around diversity, around ethnicity. I was quite surprised I came from [neighbouring borough], where there seemed to be lot of awareness about all these factors, whereas in [borough] it feels very different. Just like people aren't talking about culture 
and diversity. Like when you have a managers meeting there's no conversation about how cultural factors might be a factor in whatever the issue is. There doesn't seem to be the positive conversation around the subject, like EAL, for example. We have to bring it up but they're not bringing it up (230-238).

RHA: I had, err, an experience of an MPPM [Multi-Professionals Planning Meeting] once where, um, it was, um, they were talking about a Somali boy who- and they were talking for about 20 minutes about the issues this child was presenting with in school, and I asked the question "What's his background?" "Oh, he's moved to this country because his parents were killed in (462-465)... Somalia and he's now living with his uncle (467)...... And, I went "Don't you think we should've not started with that 20 minutes ago?!" It was almost like we've got so many of this children who've just have these complex backgrounds in other countries... (469-471).

EPs also indicated that schools could be unreceptive settings that often held very negative views about some CYP:

RHA: ... when I'm sat in a meeting with a SENCo, for example, and, and they are talking about "Oh, this parent's reluctant, erm, this parent's reluctant to acknowledge and, you know... quite often you get this with Asian parents where they're... and they make these broad...stereotypes (128130).

LA: I remember at one point I was doing this assessment with a family. I was getting a lot of background from the school about how 'they just don't engage' and this, that and the other and 'they're not wanting to learn English so they don't bother', that sort of thing. Then meeting the parent of this particular family and actually feeling like 'is that the case?' (151155)

CM: Part of our role as well is to piece together information. You might have information from the school about culture but you would want to verify that with your own information gathering, by meeting the family and finding out what's going on at home. The school isn't necessarily accurate about some things and there can be certain assumptions about different groups and particular children and families that you'd want to be very careful about your own responses to those.

\section{- Contentious Attitudes of School Staff}

EPs reported BME parents disengaging from services and/or being defensive as a result of the domineering and contemptuous attitudes from school staff: 
WL: They [school] wanted the girl to move because of her statement and her special needs, saying they wouldn't be able to meet her needs and she was going to be offered [specialist school] with a Speech and Language unit but I felt there was pressure on the parent to encourage her child to attend school, go on the bus on her own and become much more independent and it was very clear that the parent didn't want that and I felt that the climate in the room was really tense- the mum felt that school was getting her child to grow up too quickly and the use of the school bus was, she thought very much pushing her daughter to become more independent (46-54).

\section{- BME Parents with Underlying SEN}

Or parents who may have undiagnosed learning difficulties themselves and struggle to understand school systems:

WL: ... what I became aware of was that I thought the father had a language problem like his son. I think he probably didn't understand a lot of what was happening or being said in a meeting. I spoke very slowly. I think he had a language disorder. Every time he was agitated or he didn't understand and called staff stupid, what I suppose he knew best and sometimes understanding where the parents are coming from- quite often parents do have special needs themselves and knowing that can help you relate better to them. So I became aware, feel that we bridged the gap but that meeting was quite successful compared to some of the other meetings recorded in the file. (200-216).

\section{- Cultural Factors Leading to Disengagement}

RHA also indicated that the reasons BME parents disengaged from schools and/or services was due to cultural factors:

RHA: The child went back to Ghana and they got the witch doctor to see the child and like kind of I don't know what they do. I don't know. But that was very strong to the parent. They believed the witch doctor and disengaged from the services when they came back to the UK because it'd be dealt with in their way- that's very strong. But it's about the cultural construction again. That is more powerful to them in their socially constructed world than medication is in our socially constructed world (796-801).

Others stated that cultural factors and the fact that special educational needs were perceived differently by different communities had often resulted in parents refusing input from professionals: 
LA: I had a parent the other day who didn't want this diagnosis for autism and actually, when we broke it down a bit, it wasn't actually about autism; it was about everything else that might happen as a result of that (749-751).

EPs also reported that cultural attitudes towards special needs had led to parents fearing being stigmatised, experiencing prejudice and often exclusion from their community. They noted that may be the reason why some BME parents were reluctant to actively engage with professionals to get their child the support they needed:

WL: Although both parents wanted me to see the child, they refused to sign the permission slip, they requested that didn't want their child to be diagnosed as having special needs or learning difficulties or a statement but I could observe their child in class- they were worried about their child having a file that would be held by the LA. But the SENCo gave me the reason for that, that if the child had a label or a statement or a file, then that child would seen as inferior and wouldn't be able to get marriedbased on their cultural group identifying special needs as a major black mark- a stigma if you like (83-90).

CD: I think that usually the mothers have understood their child's needs and there is an issue with a wider family often blaming or telling the mother that there's no need for a fuss and there is nothing wrong with the child, that somehow- if there is a problem- the mothers are to blame for it. I think traditionally when there has been a request and a frank discussion around the child's needs, the mothers have been patronised or something along those lines. They expressed belief that it is a punishment from God - you have the original sin from Catholicism, whether it is a punishment from that - it has fallen away - when I have dug a bit deeper, that hasn't been the issue. (190-202).

\section{- BME Parents Disengaging when EPs from non-BME Background}

In addition, EPs reported that parents did not always co-operate with EPs if they were from a different background to them as they mistakenly felt that EPs would not be able to empathise with their views about what their CYP's needs were.

CT: On my placement day my supervisor told me I wouldn't be doing that piece of work because the parent had refused consent for me to work with their child for that reason, that there was a mistrust. There was a feeling that I would not be able to understand those particular needs, which I felt quite perplexed about, and actually felt quite insulted but the need was 'ok, who's next in line for that piece of work?' But that certainly opened my eyes to a different type of context and type of work (446-454). 


\section{Sub-Theme 1.3 Barriers of Communication}

Eight components emerged within this theme, including BME parents lack of spoken English, lack of shared language with parents leading to confusion, the importance of communicating with mothers, parents' limited English and accessing groups, language barrier to the assessment process, simplifying EP reports, difficulties when using labels and challenges with the use of Interpreters.

\section{- BME Parents Lack of Spoken English}

Most EPs reported experiencing parents who spoke little or no English. They stated that this was a major barrier in communication between them and the parents and, ultimately, in doing their job:

PK: ...whether they speak good enough English - that's my biggest issue really, I suppose! If I will be able to communicate with them, or not (589590).

CD: Language is such a powerful tool to reach out to all humans- it's the function that sets us apart from the other species in the world. Here in [borough], the majority of all families speak Punjabi- I would love to go and have a detailed conversation with the parents but this is not always possible as the only language we may share is English and even that is not always guaranteed. It's not good. It's not effective for me to do my job properly if I don't even know what the parent is saying or if they can't understand me (357-363).

EN: There was a child from Albania that I was working with last year, and to communicate with parents was slightly difficult because their language was quite limited and to explain some of the child's needs was quite problematic. I've found it difficult to illicit the information I wanted because they perhaps weren't understanding me (607-610).

However, even when there's a belief that parents can speak English, there was no guarantee that they would understand what was being said:

MJ: You know, you get these families that say 'yeah, we speak English at home' but, you know, and they may well be speaking English at home, but again... I lived for 20 years with somebody for whom English wasn't their first language, so that has also influenced me, and...so I have a real understanding of how, even when you've speaking fluent English for 20- 
25 years, there are still things that you miss or don't understand (254259).

One EP expanded on this and stated that it was also difficult to simplify technical psychological terms and/or explain a concept that did not exist in the parents' first language:

CT: And that is an issue because there is psychological language that we use all the time and it is tricky (579-580).

- Lack of Shared Language leading to Confusion

One EP noted how a lack of language and/or communication could lead to confusion for both parents and professionals in having a CYPs health and social needs met:

PK: I have had a situation where I got some girls where they [school] thought they were shipping them back to Pakistan to marry them off and they were only twelve and didn't have very good English skills, but that was a side line; the children were actually referred to me because of their English language, because they seemed to have speech and language difficulties. They didn't come back. I mean who knows what happened to them? Their mother doesn't speak very good English as well and has got a stutter or speech impediment, and hundreds of children, and she always says she misses these girls. And they have now come back. I don't think they were married off but nobody knew what was happening... (739-747).

\section{- Importance of Communicating with Mothers}

EPs also believed that they would get a better insight into a child's needs if they were able to communicate more effectively with mothers from BME backgrounds. However, due to language barriers, they found that it was fathers who they liaised with and who may not have had as comprehensive an insight into the child's development as the mother:

WL: I think the biggest concern is when one of the parents speak English and I tend to then... I will usually liaise with that person and that may be the father or may be the mother. When it is the father, if the father isn't the main care giver then I am aware I might not be getting all the information that I would, I will perhaps get a different picture then I would interviewing the mother vice versa. So that's the issue... (6-10). 
PK: I get a mother and father and the mother sits there, in a her, in a very stereotypical way, doesn't say anything and doesn't speak English, even though she's been here for three or four years and I feel I can't reach her and I can't ...hear, hear her views and there's the- the husband who's saying "No!" you know..."Our child doesn't have learning difficulties" (306312).

\section{- Parents Limited English Language Skills and Access to Groups}

One EP stated that a lack of English would also exclude parents from accessing local groups:

LA: And I think language probably links to that as well because, if running groups for parents, typically it's likely to be English and, I don't know, that maybe people accessing different groups only speak English and maybe that would contribute to that feeling of helplessness or not feeling empowered, not being able to express and openly communicate your views (118-122).

\section{- Language Barrier to the Assessment Process}

EPs also noted that in addition to the limitations of assessment tools and parents' willingness to share information, parents limited or lack of English hindered their ability to carry out systematic assessments with efficiency:

LA: And there's also a bit of caution in the assessments that we might do because they are going to be limited, based on some of the tools we are using, and how we are collecting the information, the language barrier and how much family are willing to tell you about what's really going on at home and what the situation really is. And that does limit the assessments and the conclusions you draw from them and the theories ((we're talking about)) around language development and how long it takes to learn English as an additional language. It makes you put on hold a little bit, how you respond to that and are we leaving it too long? Are we kind of assuming that maybe it's EAL when there are other things going on? (182190).

\section{- Simplifying EP Reports}

When parents had a basic command of English, EPs aimed to simplify their reports to make them more accessible: 
EN: You'd also be mindful of the language you do use, and in your reports, and making your reports accessible (577-578).

\section{- Difficulties when using Labels}

In particular, using labels in reports and/or in consultations caused EPs difficulty:

RHA: ... I thought this child probably had ASD, definitely had social communication difficulties, and also had learning difficulties. I'm trying to explain to that father what the implications for that were. He'd only been in the country three years, and so he was asking me whether his son was mentally retarded, and so it was very difficult then because l'm not going to use that language. But that's probably what it is called in their country. But then it might also have stigma attached to it in their country, and I don't know. So I don't (366-379).

\section{- Challenges with use of Interpreters}

EP's noted that though the use of an interpreter may be helpful, this was far from ideal. The availability of and/or arranging an interpreter to be present during meetings or an assessment was a lengthy process and one that was fraught with complications.

MJ: It takes two weeks to arrange a translator, an interpreter, because you have to provide an invoice first. Whereas, for example, a locum that's just started working with us, she thought she could get an interpreter within a day; that's her experience in other authorities (129-132).

Since the involvement of interpreters can cost schools money, schools often ask staff (i.e. a teaching assistant) who share the parents' language to be present at meetings. This raised a number of issues for EPs:

WL: I am also aware when English is the second language, often the schools use a TA from the school to translate but for me that brings up a lot of issues because of confidentiality. Some of questions we ask about the early developmental history, how happy the parent is discussing it in from the teaching assistant in the school and I never really know and I just continue my interview not understanding the relationship between the parent and the TA or whoever has been provided as a translator. (10-20). 
One EP added that though having an interpreter present at meetings did help convey some information, it prevented the EP from building a relationship of understanding and empathy with the parent:

RHA: ... it's really difficult to work productively with someone who speaks very little English. You can do it through a translator but that, that connectionyou can't read and, and that's another important point like you can't the facial expressions based on their culture might not be similar to ours... (314-317).

However, despite these challenges, it was felt that sometimes it was useful to have someone from the same language and community translating:

RHA: ...As soon as she [mother] walked out the room, the TA was like "It's not culturally acceptable, it's not what we do!" And, that boy's got very significant difficulties, possibly, largely, due to that... (693-695).

EPs reported that they frequently questioned the accuracy of information shared through the use of interpreters as they could not be sure if what had been interpreted was what had been actually stated by parents:

BFP: And of course there's a language barrier. Because a lot of them mix up, you know, they've got a mixture of languages, like French and Lingala, and something else, you know, all rolled into one. So even getting an interpreter doesn't often help (364-367).

...l've worked alongside an interpreter and l'm sure a lot of key information was lost. As it was being translated over. Yes. Well, you see, that's the problem. Some people who... like, Algerian and Arab is very similar. There is... you can't really get a translator. Because it's like... you know, once you cross that language, already they mix a couple of languages together (369-373). 


\subsection{Final Theme 2 - Culture of Schools}

Another final theme to emerge from the focus group data related to the culture of schools and the beliefs, perceptions, relationships and attitudes of school staff. Within this theme, seven sub-themes emerged, i.e. negative attitudes towards BME CYP, their parents and communities as held by schools, schools reflecting dominant narratives held in society, negative attitudes held between BME communities, schools valuing uniformity over diversity, schools negative attitudes towards BME individuals and the impact on their relationship with EPs, the power differentials between school and EPs in tackling prejudice and the power of schools to determine EP work.

\section{Sub-Theme 2.1 Negative Attitudes towards BME CYP, Parents and Communities}

A number of EPs reported that attitudes of school staff, namely class teachers and SENCos, remained unfavourable towards CYP and parents from BME communities:

CD: ...there seem to be quite strong views amongst many teaching staff particularly about the, largely the inadequacies of Somali parents in preparing their children for school... (20-22).

One EP highlighted school staff being hostile towards pupils from BME backgrounds:

BFP: ... there are very subtle ways of bullying or putting down a culture. I have a head teacher and - I've been in that school for a very long time - and to this day, if she is referring to a Muslim girl, she'll say 'oh, you know the one who wears the hijab. H-h-h-hijab' and this goes on like stuttering for three or four minutes (358-362).

School staff also worked in ways to maintain the status quo:

BFP: ...I've noticed this a lot, going around from school to school to school. The way staff and reception will deal with an Asian lady with 3 or 4 kids and a head scarf or national dress, and the way they treat another person from another ethnic group (327-330). 


\section{Sub-Theme 2.2 Schools Reflecting Dominant Narratives in Society}

Schools reflect issues that are taking place in wider society:

BFP: And of course schools directly mirror the current zeitgeist, don't they? For example, anti-Islamic views are considered not negative. It's considered not negative because there is so much of it in the media, the newspapers, the TVs or racism or terrorism or oppression of women and girls, blah, blah so that when people hold these views they may feel justified ... (395399).

One EP elaborated on this by recalling a situation where school staff indicated that two siblings with speech and language difficulties had been sent to their parents' home country to be forced into an arranged marriage:

PK: $\quad$...when I last met the mum, and the baby- who we all thought- was going to have these horrendous abnormalities was born, and was absolutely normal... so there's lots going on with the family. And then she said to me 'the daughters have come back'. One had got a place in one secondary school and one was going to the other secondary school and she was all 'oh, they've come back. I'm so happy' and I thought 'oh, well, there we are. Didn't really have to worry about that too much, did we?' And actually what could they have done? There's nothing they could have done in another country, so... (776-783).

Another EP reported a situation where school staff had overlooked a child's speech and language needs and related these to him having English as an additional language, when this was not the case:

BFP: So, recently, there was a child who'd moved in to a school from another school and he comes to the UK at the age of one and he'd been in nursery since the age of three, so he learned English since the age of three, and now he was nine, and because he was new to the school, he'd been in the school 2 years, they kept saying 'Oh, he's EAL', turned out it was wrong, he wasn't. He wasn't EAL. It was a specific knowledge and speech impairment and it was having a very negative effect on his mental health (28-34).

\section{Sub-Theme 2.3 Negative Attitudes between BME Communities}

EPs also reported conflict within-minority groups with those who were born in this country versus recent arrivals: 
BFP: Even within ethnic groups, there's a lot of competition, you know, for power and that what they would associate with white... is power and privilege and, you know, the ability to tell you what to do and put you in your place because obviously, maybe, that's something they do have experience of. You know, they've yearned to be on the other side of the fence. But I would say I've noticed that. I noticed how much contempt, sometimes, that's just barely concealed, for recent immigrants, like, from the Congo or places like this. Really and truly (348-355).

\section{Sub-Theme 2.4 Uniformity valued over Diversity in Schools}

EPs also stated that staff in schools placed heavy emphasis on uniformity over diversity and, in maintaining the established middle-class status quo.

CD: ...the best is what is the right middle class view of the best ...as such school's don't tolerate much difference, they don't tolerate much difference in terms of aspiration's for children or ways of parenting whatever... I would like it to be more than schools trying just to mould minority ethic parents to fit into what it is that schools want (52-56).

\section{Sub-Theme 2.5 Staff Negative Stereotypes and its Effects on EPs Relationship with Schools}

One EP reported always checking the reliability of information she had received from school with parents as she felt that school information was often inaccurate and value-laden:

RHA: I don't rely on what's on our files because I don't think it necessarily is always right- It's quite often the language they put down is wrong. The ethnicity is wrong. It's based on school's assumption, and so the school filled out the referral form... (146-148).

As the following comments demonstrate, many EPs also reported the need to challenge schools on their attitudes towards parents and CYP from BME communities but exercising caution when doing so:

CT: I think one of our roles in schools is to be challenging those cultural stereotypes and expectations. In schools that I work in regularly, you get to know people in a more particular way than just 'the psychologist'. The more time you spend in a school, the more you begin to understand the staff and culture and I think there are sometimes points that are made that 
are somewhat unhelpful, that you can't help but need to challenge (281286).

LA: $\quad .$. I think a lot of what I learned at university was about the language you use as a good role model and not constructing and colluding with those [negative] views but expressing what your views on it might be, or highlighting that... or maybe trying to find out a bit more about what they mean when they [school staff] say 'you know what they're [BME parents/families] like', and trying to get them to think about what they mean about it and ask them a few more questions about it like 'what are they like?' and trying to put it that way. Maybe it would be a little less direct and just get them to reflect on it a little bit (282-287).

\section{Sub-Theme 2.6 Power Differentials between School and EPs in Tackling Stereotypes}

EPs recognised the power differential of working with school staff and the confidence required to tackle any negative stereotypes:

LA: ... being a young EP, there's always going to be power differentials in different situations - if this is a head teacher saying something to you, or a senior member of staff. You might have your own views on it but it's whether you choose to share them because you might feel intimidated to do it (171-174).

LW reported the need to build trust and rapport with school staff first and stated that only then could positive change be effected:

WL: I think the key is how well you can work with the SENCo and this is the tricky bit- with some school SENCOs you can and some you can't. I think being a new EP and new to the school you have to build up the trust and relationship first and that takes a while. But once you can do that, once you have got that then you can conduct work systematically... (235-239).

Nevertheless, one EP did not feel very optimistic about schools shifting their perspective on those from BME communities. She stated:

CD: ...we have an education system which is going backwards. It is regrettable; it's only going to go from bad to worse. Many people thought that they tackled racism in education 20-30 years ago. In London, for example, there is so much stuff on anti-racism and it is all gone out of the window. So I think it is only getting worse. I think it is a tragedy; it is part of broader inequality in our society (287-295). 


\section{Sub-Theme 2.7 Power of Schools to Determine EP Work}

Within this sub-theme, four components were identified that related to school's power to determine EP work. These ranged from EPs being discontented with their current role in schools, EPs frustration with an unequal system of SEN referrals, their belief in schools unwarranted requests for standardised assessments, schools prioritising academic progress over social/emotional needs to EPs believing that it was instrumental that they helped schools prioritise the SEN referrals.

\section{- EP Discontentment with current Role in Schools}

Several EPs reported feeling frustrated and dissatisfied in the manner in which they were deployed by schools.

PK: I would say when I first started working in [borough], thirteen odd years ago, we actually had more role systemically in schools. Now, I find that, you know, it, we're, we're being referred children that are more complex, we've got less time in schools now with the way we're working with two sessions in each school, whether it's a massive primary school or a tiny primary school. And, so we're not, we don't have the privilege of working at the systemic level (494- 499).

PK: ...the children who are underachieving maybe because of their cultureschools need to tackle those themselves, they need to be the ones that are looking at those, those children's achievements and you know, putting things in place for the whole group of them. We're the ones that are maybe looking at those individual children below that (515-521).

\section{- Frustration with Disparate System of EP Referrals}

EPs discussed that when schools bought in EP services, this benefitted some and not all pupils- though the latter may be needier:

EN: One of our out of borough schools only buys five hours so that's, so some of these different populations are not getting the full quality EP service. Some schools buy a day a week or something like that (253-255).

CM: Also, some schools would choose to use that time in a particular way. They might want more diagnoses of dyslexia. Maybe if you look at the 
requests for our involvement with those children there could be a particular demography associated with that group too... (260-263).

\section{- Unwarranted Use of Standardised Assessments}

EPs also reported that standardised assessments were not always the correct response:

LA: And I think sometimes with children who might be arriving in the country, there is not actually that much information on their learning all together, so that can be a difficult one. Schools want a starting point [psychometric assessment] and... ethically, that's a difficult one as well (522-525).

WL: I can see there is the pressure to do cognitive assessment straight away but it's not always possible or in the best interests of the child and it doesn't always help you get to where you need to be (309-314).

\section{- EPs Instrumental in helping Schools Prioritise Referrals}

One EP stated that there was a need for EPs to play a bigger role in schools, in advising staff which information was necessary to support an application for an assessment of a CYP:

MJ: I think the really important thing is for schools to know what information they should be collecting as the bare minimum. Have they thought about what the child's first language is like? Have they interviewed parents and spoken to them about that? What are they doing and how is the child responding to it? (537-544).

Although some EPs felt that it was important to challenge schools on some of the referrals they were making:

CD: ...we should monitor, we should think about what numbers of girls and boys of different minority groups we are reaching, which children would really benefit from our input. (302-304)...we need to challenge schools and question if we are we targeting all those particular groups of children or are we letting black boys slip through the net- we need data from the schools as evidence to help us shift our practice and prioritise those in need (309-312). 


\subsection{Final Theme 3 - Changes to Education Services}

Within the final theme of Changes to Education Services, four sub-themes were identified. These included cuts in educational services and limited resources, the impact of service cuts on EPs, changing perception of diversity and the changing nature of EP work.

\section{Sub-Theme 3.1 Cuts in Educational Services and Limited}

\section{Resources}

EPs noted that many LAs had dispensed with vital educational services and roles:

MJ: $\ldots$ all the minority ethnic teachers have been decentralised, it's up to schools to buy them in. And the EAL teachers, those centralised services have gone and I think schools have now taken ownership of those issues and there is not somebody keeping it high on the agenda. So I think there is a lot of dodgy practice going on there (139-143).

This sub-theme illustrated how changes to the wider system affected the EPs role when working in a multicultural community. The issues here were not located in the community but situated in the evident changes taking place in LAs:

LA: I get the sense that, because there are so many families that need those services, that that just puts a big strain on resources, which surprised me because I thought, with there being more families, there would be more resources, but it seems a bit stretched (156-159).

\section{Sub-Theme 3.2 Impact of Service Cuts on EPs}

Within this sub-theme, three strands emerged: the impact of service cuts on the role of the EP, the impact of service cuts on EPs working ethically and the impact of service cuts on EP time. 


\section{- Impact of Service Cuts on the Role of the EP}

As a result of the cuts in educational services, some EPs felt that the onus now fell on them to ensure that they were providing essential services as well as accompanying and/or sign-posting parents to other services and/or agencies. This, they felt, increased their workload and resulted in limited capacity to complete core work effectively.

RHA: I signpost them to people who can help them do that. But we're not... My understanding of the EP role is to identify the needs of the child and family if that's relevant, and it might be that your... that that family aren't acknowledging their child needs, a great deal of support, or that they're perhaps not managing that child's needs appropriately. And so, yes, then you might be signposting them to agencies that can help them from that perspective or help advocate for them. But I've been told to back down a few times when l'd been advocating for families (323-331).

\section{- Impact of Service Cuts on EPs working Ethically}

EPs in certain authorities highlighted their own experiences related to their particular settings and felt that cuts to services had resulted in some EPs adopting unethical ways of working:

MJ: I have worked in a service that used to do psychometrics, verbal and nonverbal, some tests, on anything that walked or crawled, or any person that walked or crawled, regardless of their bilingual experience, for example (190-193).

Similarly, there appeared to be reluctance from EPs in this particularly overstretched service to demonstrate flexibility in their way of working and adopt a more ethical approach to work:

MJ: ...there's a culture... there's this group culture of, 'no we'll just carry on doing what we do', and as the leader of the group it's my responsibility to change that, but I have to admit, actually thinking about it even more, I have to admit I do struggle and I sometimes lose the energy to maintain that; constantly questioning and drawing the team away from that common way of working and to be working in a more ethical way (322327).

EPs cited one reservation with traded services and noted that it could lead to issues of inequality and unethical ways of working: 
CT: We were working there with an increasing number of faith schools, so Muslim schools, Catholic schools, Church of England schools. All the schools had a faith attached to them and, with all the Free Schools and academies, are we in danger of going even further down that road so children's experience of school is increasingly not representative of the diverse society in which they live? (602-606).

\section{- Impact of Service Cuts on EPs Time}

Other EPs complained about the lack of EPs in services, increased workloads and lack of time to further explore the diversity of the local BME population:

MJ: ....in the last 5 years... there have been cuts, you know, austerity measures. People are rushing and doing things more quickly and they haven't got the time to check out information, and so... so there isn't necessarily the time to make a point of finding out about different cultures or different cultural groups. Does that make sense? (298-303).

\section{Sub-Theme 3.3 Changing Perception of Diversity}

Two distinct strands emerged within this sub-theme, i.e. changes in monitoring diversity and change in LA values.

\section{- Changes in Monitoring Diversity}

Although EPs valued diversity, many noted that recent changes in monitoring BME diversity within their services had decreased due to a lack of direction from mangers:

MJ: Yeah. But it's hard when you don't get the reinforcement from your own line managers. When it feels like the culture of the organisation is not to make sure we're meeting the needs of diverse communities. I think we're quite good on disability, but this is very much children with physical and learning needs, but in terms of culture and minority ethnic groups or different linguistic cultures or whatever, then... (565-570). 
However, there appeared to be variation between services, with some local authorities actively collecting data in order to respond to the needs of the local population:

EN: I think we need to respond to that local need. We're all based on locality teams and we get data from these locality teams, based on exclusions, things like teen pregnancies, drug and alcohol... and those data do break down into certain ethnic minority groups; we are aware if a certain population does start...it's about being responsive to those individual populations, so we do have data on a yearly basis, so we can respond to that (500-505).

\section{- Change in LA Values}

EPs also noted the cuts in services had resulted in certain principles, which had previously been upheld, to shift dramatically. For example, one EP reported that with the cuts that had hit the service, a number of professionals, including himself, were not being challenged enough and were focussing on just the basics of their job and nothing more:

MJ: And I think what I've noticed, and this maybe relates to a previous thing, is that I'm not being challenged by my line manager. My line manager's never said to me 'what's the proportion of children referred to the EPS who are from minority ethnic groups?' I'm not saying... I'm making excuses now, I'm making excuses, but it's very difficult to keep all those plates spinning at the same time (327-233).

\section{Sub-Theme 3.4: Changing nature of EP Work}

Three components also emerged within the changing nature of EP work subtheme. EPs reported their dissatisfaction at the way in which EP services had changed to a traded model of service delivery and ethical and moral dilemmas of this in relation to BME pupils.

\section{- Traded Services}

Many EPs expressed their discontent with the nature of EP traded services. One added: 
CD: I think the important things to be an EP are humility, a commitment to social justice and social equality. I don't think we should be on the fence. I don't agree with private EPs, I don't believe in traded services...I don't think it's right but... a lot of my colleagues believe that traded services- so the privatisation of a service that is meant to be there for all- is the way forward. I absolutely detest the changing nature of our work- our clients within traded services are the schools who crack the whip and we then answer to them and pander to their needs, instead of the very children who I came into this profession to serve. That disgusts me (412-419).

\section{- Dilemma-School vs CYP as EP Clients}

In addition, traded services highlighted the issue of who the client was- the school or the child:

CT: ...the real risk, I think, of where we are at the moment is that question about who our client is- and the child certainly should be at the centre of that- but I think there is some risk that when it is somebody else who's commissioning that service - who is paying for that service. Does that have an effect on who our client is? Does that mean our client is actually the school? (153-157). 


\subsection{Final Theme 4 - EP Training}

Another final theme to develop from the focus group data was that of EP training. This theme comprised of five sub-themes ranging from opportunities to work with diverse communities, training with a diverse cohort on the EP training course, need for diversity within EP training staff and services, training on diversity within EP courses and the need for a shift in training curriculum for new EPs.

\section{Sub-Theme 4.1 Opportunities to work with diverse communities}

Some EPs noted that their EP training course equipped them with an awareness of working with diverse communities:

EN: ...one of their course requirements in year one was to have a piece of work that involved working with ethnic minorities (469-470)... So that's just one institute. I was at [EP training provider within a culturally diverse London borough] as well (473)... I do feel there was a priority in working with children and families from different backgrounds (474-475).

CM: ...from the very beginning you realise there's a big emphasis on Equal Opportunities because one of the questions in the interview is about Equal Opportunities... (476-478)... I remember having lectures from a man about the high rate of exclusions of young black men in schools in the UK, so there were certain topics covered that I think helped us understand a bit about some of the issues we might encounter (480-483).

However, other EPs had a different view of their training and its commitment to promoting diversity and equal opportunities:

RHA: ... it was such a rarity to get a ca- $\ldots$ but if I got a case and, it was, [laughs nervously] they were from a kind of different...cultural background, it would be a rarity- I think it happened twice in my whole three years of training... (372-374) ... it was a box that was ticked in my portfolio! (376).

Another EP noted that there was a difference between what was being taught on the course in relation to diversity and equal opportunities and the variation of experiences on placement. This resulted in some trainees who had a lot of experiences of working with CYP from ethnic minority communities to those who had very little: 
LA: I worked in an area where there is a high percentage of children from minority backgrounds, compared to other schools in the area. So I had quite a lot of experience and I think I saw, potentially, the gaps between some of the things I was learning from experience, compared to some of my peers I was training with. And I don't feel that maybe the course touched on some of these things until I actively went out and found that information (77-82).

Another EP noted parallels to LA in his training experience in London:

CT: I'm still surprised now how weak I feel my training for this job was in general and in so many ways, it is left to your experiences as individuals on placement to learn I suppose than actually being taught. And I think that we have a very limited range of experience on placement (771-774).

LA emphasised the need for more discussion around the study of diverse groups due to an increased growth of certain minority communities:

LA: I got the sense that this is something we haven't talked about before and is something that we might need to discuss more, because of the changing population (82-84).

\section{Sub-Theme 4.2 Opportunities to train with a diverse cohort on EP training courses}

Some EPs commented on the lack of diversity on their training courses and highlighted the unrepresentative nature of professionals within services:

LA: There was, I think one member of staff who was of Asian origin in my service and he used to feel strongly about and got very involved in a lot of the work that's done... (94-95)... And the same in schools, as well: very white, British teachers, working in communities with like $98 \%$ Asian background and origins. I think that it's important to have a range of people of different backgrounds and experiences but, for some reason, maybe it's about who applies for it (96-100).

RHA: ...our course wasn't culturally diverse $(575-576) \ldots$ We had [trainee] who was from... I think she was Iranian. But that was it really in terms of diversity. And she was Muslim. So she did sometimes bring in into our discussion that extra element... (580-581)... But it does help in your discussion to have people from different backgrounds because that's when you do a lot of your learning during those tutorials and when you're talking about issues. If you can ask someone and say... And she did quite 
often say, "But from my perspective, that would be okay." And so it really stopped you in your thinking. (584- 587).

EPs elaborated on the benefits of training with peers from diverse cultures and backgrounds. They stated:

WL: The group that I was with was quite diverse- it had a good mix of different cultures and it was also a challenging group in that we were challenged a lot in our own belief systems... I think the fact that I worked alongside Black and Asian colleague as well, was really helpful -to get their views and their takes on their experiences working in schools and how they were received. I found it very helpful of course and very challenging ...I think again sometimes as a white person you can be unaware of the racial issues and tension of less...people who are unlike you in many ways. When it's highlighted in front of you... it makes you ask what your own beliefs are. I think you typically know that but being next to a black person when they are experiencing the subtleties of racism I think that's when you get the real experience of the way the world can be so unfair. I find that the white person can often overlook challenges experienced by non-white people... unless it's specifically bought to their attention (254- 265).

CD: ...we had a nice, diverse cohort. We had males and females; people from more working class backgrounds; there were at least three black women on the course. I felt that I was in a setting where there was a commitment to equality and I felt it very different from some training courses where they all seemed to be quite privileged. I may be wrong but that was my stereotype: all white, middle class, young women, and I was so glad that I was at [training course within a culturally diverse London borough]; I thought I belonged there. Honestly, I think I would have given up if I had been somewhere else because I wouldn't have felt like I belonged, whereas in my particular course, my particular year, I thought 'I'm like a lot of these people. I belong here, it's OK, I can do this, I can be this person'. And this is back to your point about seeing yourself in the situation and I think parents, when they see someone doing the job who's not just like everyone else, it gives hope and a sense of belonging and a right to be there. Very important (515-527).

\section{Sub-Theme 4.3 Promoting Diversity within EP Training Staff and}

\section{Services}

A related issue was the lack of cultural diversity amongst tutors and/or speakers on the course:

RHA: ...our tutors were white middleclass or upper-class themselves, so I think... (587-588)... If we would have just had more speakers from 
different backgrounds because we were lacking that diversity on the course, it does extend your thinking... (588-590).

WL highlighted the importance of promoting diversity within training course staff by stating:

WL: Even speaking to one colleague, I remember saying when she got on the course, she was Asian, and she said that she was so pleased to see that one of the tutors was black because it made me feel much more relaxed. And just that comment makes me aware that, while I wouldn't have considered it either way, for her it was very important that one of the tutors was black. So it's just by working alongside colleagues that you become more aware of these issues (272-277).

CD noted the importance of promoting the EP profession to individuals with a diverse range of life experiences:

CD: I feel very strongly that we should have a workforce that reflects the population we work with- the people that I work with and alongside absolutely have a duty to promote inclusiveness and equality of access- I think we should strive really hard to encourage people to come forward into the education field- not just those from a white middle-class British background but, you know, people from working-class backgrounds and other minority ethnic backgrounds (344-349).

EPs further highlighted the need to challenge the lack of cultural representation on EP training courses:

CD: As an EP team, it's important to have those who are dissimilar to us in terms of race, culture or backgrounds- it means we would be able to share resources, good practices, we'd be able to talk about things, we could challenge one another. Although it doesn't feel very nice, I think we'd be able to develop our personal and professional practice through having our prejudices challenged by one another. More needs to be done to recruit a diverse group of EPs for the next generation (350-356).

CT: We do our best in those areas that we can have influence in but what is required is more BME EPs, we need to be opening up the training to more people; it needs to be easier to get onto the training (583-585).

However, one EP believed that there may be a political explanation which may highlight why individuals from BME backgrounds did not apply for EP training courses: 
CM: There's a huge inequality of opportunity in the UK, I think, most of the top jobs go to people from private schools and there's a lot of nepotism. If you look at the cabinet, for example, it's by no means diverse and they are pretty much all privately educated, many went to Oxford and Cambridge. I think in terms of representation of ethnic minorities who are Educational Psychologists or who work in education, and teachers, I think that might be similar in the teaching profession too, I think part of it is probably because there aren't enough people who've been from their cultures who've been almost role models in the first place, perhaps (303-319).

\section{Sub-Theme 4.4 Training on diversity within EP courses}

EPs also reported their belief about how the psychological theories, models and training on using specific assessment tools within courses were intransigent and based on a 'one-size-fits-all' approach which did not necessarily reflect the needs of a rapidly increasing diverse population.

LA: We're having an interesting discussion, actually, in the service, about KOLBs model of language development, bilingualism and how one colleague has been quite critical of that model. It just made me think, actually, it's a beacon piece of research that's used quite a lot by psychologists and educational establishments, about what good practice for the EAL is. Her perspective is that she's done some research on that and really critiquing who they did that research with and if you can apply that across lots of different cultures and languages. It made me think that because there isn't actually that much research out there for us to draw upon, in terms of language development, and there is a limit to how much we can apply in terms of psychology and EAL, I think (78-87).

One EP felt that relying on Western psychological models with a BME population was one-dimensional and thoughtless:

RHA: ...you'd be practicing very one-dimensionally if you were just applying Westernised psychology if you like, and you weren't understanding all the other dimensions. I think, yeah, that would be a bit thoughtless really (729729).

LA: $\quad .$. from an assessment point of view, I think we are limited to the tools that we have to work with EAL populations... (502-503)...I think even the tools that are out there that don't have as much language demand, they are still limited (518-519).

CD: ...the way we work as EPs, whether we articulate it, whether we're conscious of it or not, we work within very dominant models of what's good 
parenting, what's appropriate for, for people to do with regards to their children's education. They are quite narrow models and so other communities, who don't have those, whether it's to do with social class or ethnicity, don't fit into that, they're found wanting... (104-108).

\section{Sub-Theme 4.5 Need for shift in training curriculum}

One EP highlighted the need for EP training courses to update their curriculum to reflect the changing nature of the education system:

CT: I mean the pace of change in education has been extraordinary in the past three years. It's the one way you could argue that the government has been effective, all be it in a very dangerous way, an untested way. That couldn't have been reflected in our training course because the context has changed, the economic effects of 2008 have completely changed everything since that time. But I do wonder how much the EP training course has changed. It sounds very much that people in the first year at the [EP training provider in London] now are doing exactly the same stuff that we did seven years ago (511-521).

CD stated, there was a need for EP training courses to shift their focus in the way

they expected future EPs to work and meet the needs of diverse communities:

CD: I think community psychology is fantastic; I've been to quite a few seminars on community psychology and the psychologists there are doing brilliant work- not just here but all around the world in all sorts of communities. I'd love to be a community psychologist but who's going to employ me? This needs to be something training courses need to focus more on, especially with the new wave of EPs. Absolutely (396-401). 


\subsection{Final Theme 5 - EPs Role Working in a Multicultural Society}

A final theme to emerge from the focus group data was what the role of the EP entailed when working with a diverse school population, whose 'race', culture and ethnicity was different to their own. Within this theme, three sub-themes emerged; EPs approach to issues around 'race', culture and ethnicity, the importance of inter-personal skills when working with BME families as well as advocating a social justice approach of working with BME families. These three sub-themes are discussed in detail below.

\section{Sub-Theme 5.1 EPs Approach to issues around 'Race', Culture and Ethnicity}

This sub-theme consisted of three components, i.e. EPs feeling that they lacked confidence and experience in dealing with CYP/parents whose 'race', culture or ethnicity was different to their own, the politicisation of issues around culture and religion that gave rise to EPs feeling they should exercise political correctness and, EPs using their BME colleagues as a source of support in addressing issues around 'race', culture, ethnicity and religion.

\section{- Lacking Confidence and Fear of Offending}

EPs reported feeling cautious when interacting with those from BME communities, lest they caused offence:

CT: I think there are times when I think I'm a little out of my depth... (312-313).

PK: There's a deep-rooted fear of offending... (333) ...it's that fear of the unknown (380).

NA: ...and it's a shame that you feel you have to tread on eggshells around it because... we're not going to suddenly change the population of EPs, this is who we are and you should be able to, erm, you know, feel confident to discuss these issues... (216-218). 
RHA: ...I probably will tip-toe around issues like that because I am not Muslim, I don't come from a Muslim background and I don't know all the, kind of, the implications of that... (201-202).

\section{- Politicisation of Culture and Religion leading to Political Correctness}

Other EPs reported feeling overly-sensitive discussing/exploring topics with BME parents due to politicisation of certain issues around culture and religion:

PK: I know it's such an issue that, , that, some people are very, ... we all have such different views about it that... you know, we say at dinner parties that there are two things you don't talk about- politics and religion! (102-104).

CT: I am aware of it and I'm certainly aware of situations where I don't feel entirely comfortable. I'm working in special school with a higher proportion of what would be representative of the rest of the borough of children from ethnic minority backgrounds. This does inevitably mean that sometimes I'm in situations where I'm not quite sure how to respond and I've probably had to take a step back and think about that (303-305).

LA: I feel quite comfortable talking about my own. I sometimes do feel that I need to be careful about how I speak about other cultures, race and ethnicities because there's so many political things going on, particularly in our educational world. There's so much, kind of, inclusion and diversity, equality, you know, and we're assessed on that all the time. I hope that I wouldn't be saying anything that wasn't politically incorrect but you don't always know if you are using the right language... (290-297).

However, adopting a politically-correct approach was not always seen as the appropriate course of action:

RHA: ...there was that one case where perhaps I was walking on eggshells whereas a colleague who's from a Muslim background was outraged by what a child had said. And so that's important really because, for me, I might not have picked up on something that was quite an extreme view because of my own awareness of being politically correct and sensitive to the culture, but actually, that's probably ill-informed because my knowledge isn't that great (6-10). 


\section{- Using BME Colleagues as a Resource}

One EP noted needing reassurances from colleagues belonging to the same cultural or ethnic background as the BME families she worked with in an attempt to further explore cultural norms and to inform her own practice:

RHA: ...l've sat next to [BME and Muslim colleague] for the last, what, six months, and l've used it as a form of knowledge for developing my understanding of Islam, and the Muslim religion... (439- 440)... Because again, I'm acknowledging that I haven't come from that background. I've come from a very different background to the place... to [borough], and so I love sitting next to [colleague] and finding out so much about religion and finding more about what's cultural and I'll ask that. And I feel safe to be able to ask that... (442-445).

... [EWO], for example, is Ghanaian, and I quite often ask her lots of questions about like, "Oh. Well, is this normal? Is this... What do you think about this?" and sometimes, she'll be like, "I don't know, X," but sometimes, she'll quite often offer like a real insight into that culture, which is really helpful for me (509-512).

\section{Sub-Theme 5.2 Importance of EP Inter-Personal Skills when working with BME Families}

Some EPs noted that asking questions was essential in developing a shared understanding between BME parents and professionals so that it brought about positive change in the outcomes for a CYP. They believed this was where an EP's inter-personal skills (e.g. active listening, empathy, being non-judgmental, showing respect) came into play.

EN: You try to use skills like empathy, listening; skills about letting the other person tell their story; you restate things so the other person feels heard; you try and do some background information about what's going on, so you're not coming in and getting them to answer the same old questions again (569-572).

LA: It's very important parents feel listened to and totally respected if they say 'in our culture we don't believe this and this' and that's a really important starting point for a conversation. It's not a barrier. It's like saying 'well how do we join up the two things?' So here we are from our cultural perspective, trying to somehow do good. Let's try and meet in the middle (206-211). 
RHA: ... a colleague..., I remember when she wrote a report, and it was a family who had recently come over from Africa. I'm not sure what part. And I think it might be Somali family as well actually. She did a home visit, and the report was framed on the wall (298-300)... I think that just speaks volumes sometimes for... "Do you know what? We're being listened to. We're not being kind of excluded from society for our child's difficulties" (302-304).

\section{Sub-Theme 5.3 Advocating a Social Justice Approach of working with BME Parents}

Many EPs reported a sense of social responsibility towards BME parents:

CM: I think, perhaps, when l've worked with parents who have had difficulty expressing themselves in the way that they'd wish, that's made me conscious about my responsibility to represent their views as accurately as possible... (16-18).

EPs also conveyed an awareness of the multiple systemic factors (e.g. socioeconomic status, cultural hegemony, racism) and the environmental contexts in which BME families lived:

BFP: I think a thought that I always have is about socio-economic factors and experiences of powerlessness in people from ethnic minority backgrounds (101-103).

WL: I think creating a voice is very important for demoralised and/or vulnerable groups... make their voices louder- their views are very important (179181).

Despite this, EPs felt that demystifying their role through joint-working and involving BME parents in the decision making process empowered BME parents in making good decisions for their children:

RHA: I think it's so important that parents are empowered to make good decisions about their children and they are included as a part of the team (746-747).

LA: I also think politeness is always so crucial to make people feel included (730) ...to show acknowledgement that it isn't one person wielding power over another; it's going to be collaborative and your voice is going to be heard (736-738). 
MJ: $\quad .$. l think that's what we are good at, kind of breaking things down in that way and making sure that it's in the kind of language that someone can make sense of and then they are more empowered to make the decisions and make the choices (752-755). 


\section{Chapter 5: Discussion}

\subsection{Overview of the Chapter}

Using a social-constructionist framework, this research explored the views of EPs on the challenges they faced when providing services to CYP and/or families whose 'race', culture and ethnicity appeared different to their own. Now findings will be considered in the context of this research question, followed by a critique of the methodology and a conclusion with the potential implications resulting from the research.

\subsection{Final Themes relating to Research Questions}

This section will discuss the association between the final themes identified and the research question posed. Figure 2 shows the relationship between each of the five final themes, which are represented using a reconceptualization of Brofenbrenner's ecological systems theory. At the centre are three interacting themes within the microsystem: EPs reflection on role, their training, and the challenges working with BME families. These three themes happen within the mesosystem, the culture of the school. The school's culture is in the broader exosystem of changes within the education system. By seeing the systemic relationship between the final five themes it is possible to see how change can occur. 
Figure 2: Relationship between the Five Final Themes Identified:

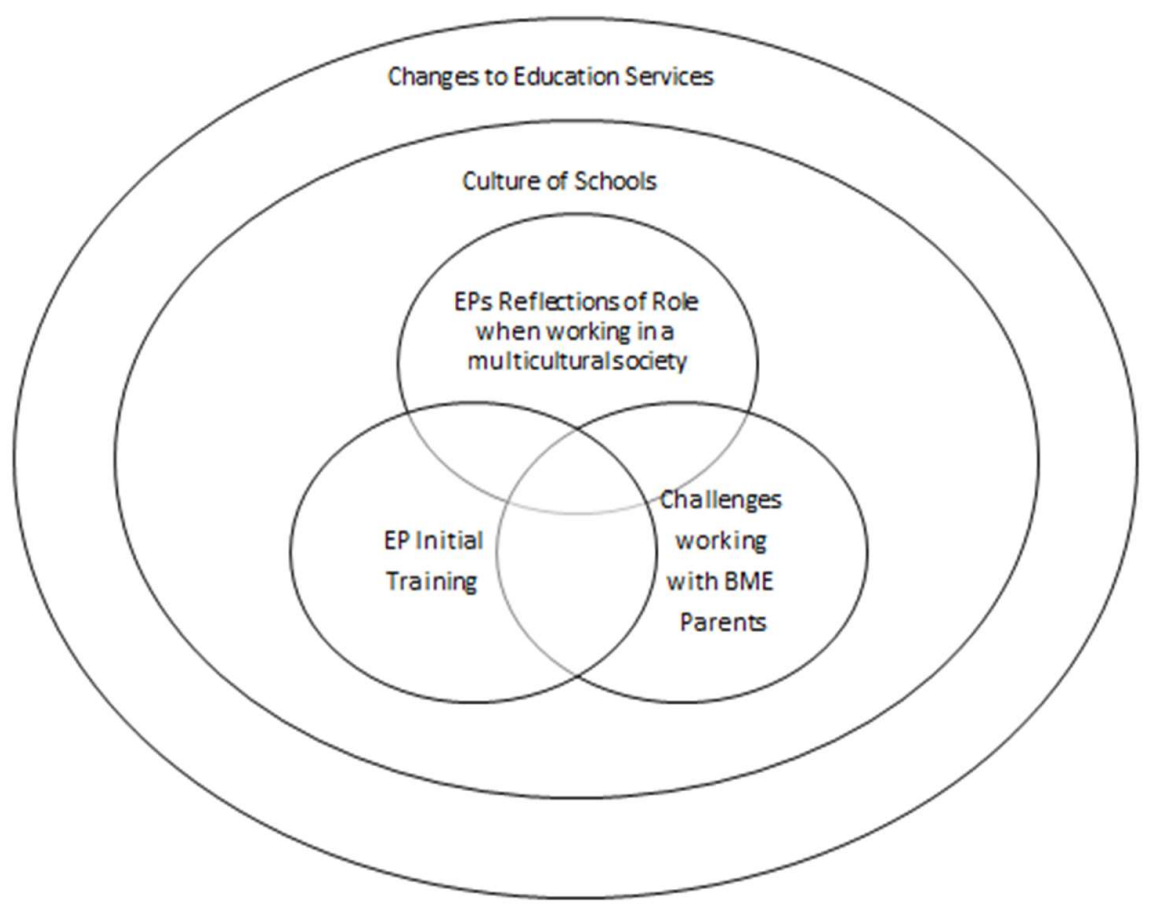

\subsection{The Microsystem}

\subsubsection{Challenges of working with BME Parents}

The first theme identified in the analysis related to the challenges faced by EP when working with BME parents. Here, EPs reported, at length, the difficulties of working with BME parents whose norms of parenting, learning and achievement differed to their own, the defensiveness and disengagement of BME parents as well as the barriers of communication. As identified in the literature review, although the area of multicultural assessment has received great attention and has been widely researched (e.g. Miranda, 2002; GoPaul-McNicol and ThomasPresswood, 1998; Hosp and Reschly, 2002; Goh, et al., 1981; Stinnet, at al., 1994), specifically pertaining to the assessment of bilingual pupils (e.g. Ochoa, et al., 1997; Winter, 1999; Figueroa and Newsome, 2006; Gutierrez-Cullen and Kreiter, 2003; Cline and Shamsi, 2000; Goldstein, 2004; Oller and Eilers, 2002; Cline and Frederickson, 1996; Figueroa, 2002, etc.), it is interesting to note how little the EPs in the current study identified issues with psychometric testing as a major area of concern. 
Since the majority of the research on multicultural issues, i.e. assessment, originates from the US, this finding could be explained simply in terms of the differences in the composition of the BME population in both countries and the subsequent differences in experiences of British EPs and their American counterparts. Similarly, it should be noted that 'race' and racial inequality have powerfully shaped the US and the UK in different ways, therefore the experiences, thoughts and behaviours of the BME population between the two countries is also likely to differ. It may be that BME parents in the US are more engaged and proactive when working with school psychologists, allowing practitioners the opportunity to conduct detailed studies of the BME population in relation to psychometric assessments.

From the current study, it appears that EPs in the UK do remain somewhat apprehensive of the assessment process of those from BME or linguistically diverse backgrounds. However being frontline professionals, who are directly involved with CYP, families and schools on a daily basis, they face a number of additional barriers that precede the process of assessment which they feel impacts on the overall assessment process of a BME CYP. This finding also highlights that educational practitioners and policy-makers should be prudent when generalising multicultural competence proposals and strategies from literature in the US to the UK population.

\subsubsection{EP Initial Training}

This theme highlighted EP initial training opportunities to work with diverse communities, opportunities of training with a diverse cohort on EP training courses, promoting diversity within EP training staff and services, training on diversity within EP courses and the need for a shift in the EP training curriculum as salient issues.

EPs stated that having BME trainees also helped other non-BME trainees on the course and noted that the presence of trainees and teaching staff from BME communities had enhanced their ability to relate to their BME colleagues and been instrumental in them providing appropriate services in their practice with 
CYP and families from BME backgrounds. In addition, EPs also emphasised the pressing need for training courses to be updated to emphasise culturally competent practices (e.g. Canter, 2006) as they felt that issues around multicultural issues were not adequately covered on their training courses (e.g. Ochoa, et al., 1997; Loe and Miranda, 2005).

This theme supports a number of literature reviewed, in that, it highlighted an essential need to increase trainee educational psychologists from BME communities (e.g., Curtis, et al., 2012; Clark, et al., 2012; Davis, et al., 2004; Fagan, 1988; Curtis, et al., 2004) as EPs felt that this would benefit CYP and families from BME backgrounds should they express a preference for an EP of a similar background (e.g. Whaley, 2001).

\subsubsection{EPs Reflections of Role when working in a Multicultural Society}

This theme related to the EPs' reflections on their role when working in a multicultural society. This included the EPs approach to issues around 'race', culture and ethnicity, the importance of EP inter-personal skills when working with BME families and them advocating a social justice approach to working with BME parents.

This theme provided some support for the literature on consultation and intervention in that EPs reported the benefits of adopting a consultative approach when working with BME families, i.e. rejecting the need to be perceived as an expert and working, instead, in a way which allowed EPs to work collaboratively with others, enabling them to solve their own problems (e.g. Larney, 2003; Wagner, 2008) to bring about positive change (e.g. Cording). In addition, the EPs in the current research drew on the principles underpinning several consultation approaches when working with those from BME communities. The EPs recognised the need to use interpersonal skills to encourage the active participation of service users and to have an awareness of the dynamics present in relationships between service users and practitioners. In addition EPs highlighted the importance of critically reflecting on their practice and considering 
alternative ways of working. They demonstrated an awareness of the educational and emotional factors that facilitate or impede the provision of effective teaching and learning for CYP as well as showing an understanding of the factors that result in underachievement, disaffection and social exclusion amongst vulnerable groups. In addition, when EPs worked with marginalised individuals from BME backgrounds, they reported doing their utmost to empower them by disseminating information about the structures and systems of many settings in which education, health and care were delivered for children, adolescents and young adults, including child protection procedures, so that parents were able to make informed decisions about the future of their CYP.

A major criticism in the literature review on the use of consultation approaches in schools pertains to school psychologists using consultation models from the field of counselling psychology (e.g. Sue, et al, 1992). However it seems that in the absence of multicultural consultation models and approaches advocated for the use in school settings (Ingraham, 2000; Henning- Stout and BrownCheatham, 1999), EPs seem to have had little choice but to turn to the field of counselling psychology, since it is the forerunner in engaging with issues related to multicultural research and theorising (e.g. Lopez and Rogers, 2001; Rogers and Ponterotto, 1997) and, to use an eclectic mix of multicultural competencies to tailor-make a consultation session to meet the specific needs of consultees.

One such approach appears to be using competencies as outlined by Rogers (2000) in which practitioners are encouraged to be mindful of their own and the culture of the consultee and develop their cross-cultural communication and interpersonal skills. In addition, acquiring culture-specific knowledge is also endorsed to ensure the effective delivery of consultation services. 


\subsection{The Mesosystem}

\subsubsection{Culture of Schools}

Another significant theme to emerge from the analysis was the culture of schools. EPs drew attention to a number of issues that they had to contend with when their work involved working with BME families. These included negative attitudes from staff towards BME CYP, parents and communities, schools reflecting the dominant narratives in society and valuing uniformity over diversity, the negative stereotypes of school staff towards BME families and its effects on EPs' relationship with schools, power differentials between school and EPs in tackling stereotypes and the power of schools to determine EP work.

Although the literature review did not highlight this, this finding gives credence to other research that states that in addition to educational inequity, those from BME backgrounds face additional challenges at school including low expectations and labelling by school staff (e.g. Ajegbo, et al., 2007; Sukhnandan and Lee, 1998) as well as institutional racism (Macpherson Report, 1999). It could be argued that this potentially results in a paradox of further BME educational underachievement ensuing from BME pupils internalising the negative stereotypes to which they are subjected (Cline, et al., 2002). In addition, this finding also indicates that schools may be struggling to acknowledge and/or accommodate the demographic changes that have seen the proportion of BME pupils increase rapidly. Moreover, with their already fast-paced and stressful roles that invest time and resources in pupils to boost performance and subsequently the status of the school, school staff may feel unprepared to directly tackle the diversity in languages, cultures and ethnicities and may perceive these as additional stressors. This may result in them becoming dismissive and/or contemptuous towards those from linguistically, ethnically or culturally diverse backgrounds. Alternatively, it could be that like the EPs finding it a challenge to validate the differences in the norms of parenting, learning and achievement among BME parents, teachers; seen to be agents of the state who promulgate middle-class norms and values, may do so too. This clash in cultures may result in symbolic violence; criticism/verbal attacks from school staff towards BME CYP and families for not having or knowing the right manners, accent, confidence and knowledge (Lynch and Baker, 2005). 


\subsection{The Exosystem}

\subsubsection{Changes to Education Services}

Although changes to education services was not highlighted in the review of literature, EPs reported at length the cuts in educational services and the limited availability of resources, the impact of these on EPs, changing perceptions of diversity within LAs and the changing nature of EP work as key issues affecting their ability to work effectively with those from BME families.

This finding may be attributed to significant changes that have taken place within the EP profession in recent years. For example, the austere economic climate created by government to reduce public expenditure to protect fairness, growth and economic stability for the longer term has led to local and national budget cuts to public services. In addition, legislative changes, e.g. The Children's Act (2004), The Academies Act (2010), The Education Act (2011), The Children and Families Act 2014 and the Special Educational Needs and Disability Code of Practice (2015) have had substantial implications for Local Authority (LA) Educational Psychology (EP) services across the UK (Association of Educational Psychologists [AEP], 2011; Morewood and Rumble, 2013). With LAs facing substantial cuts in funding from central government, the majority of EP Services have adopted 'traded' models of service delivery (AEP, 2011) in order to preserve jobs and protect services.

However, as pioneering as the traded approach to service delivery may be, it is an area that has received little attention to date (Allen and Hardy 2013). The AEP's guidelines on the Principles for the Delivery of Publicly Funded Educational Psychology Services (AEP, 2011) state that access to the service should be free at the point of delivery to children, young people and families. However findings from this study (Final Theme 3: Changes to Education Services, p. 88) suggest that in the current market economy, EPs are facing ethical dilemmas concomitant to traded services which are impacting on their relationship and ability to work with other LA professionals and services, school staff, CYP and their families- particularly those from BME communities. The EPs felt that the recent changes in LAs in relation to monitoring diversity and change 
in LA values had resulted in managers further relegating their priority in meeting the needs of the local BME community.

\subsection{Methodological Considerations}

This section provides a critical review of methodological issues within the current study. These will be discussed under the following headings: sampling method and data collection and analysis.

\subsubsection{Sampling Method}

The purposive sampling technique relies on the judgement of the researcher to select the individuals being studied "based on a specific purpose rather than randomly" (Tashakkori and Teddlie, 2003a, p. 713). In this case, the recruitment of participants was limited to specific outer-London boroughs; identified as being some of the most diverse local authorities in London (Census, 2011). Given the time constraints of doctoral research, it was felt that purposive sampling would enable the me to make use of the experiences of this population "that cannot be gotten as well from other choices" (Maxwell, 1997, p. 87) quickly and easily. In addition, when considering the motivations of the participants who volunteered to take part in this study, it may be that those who had a personal and professional interest in the topic were more inclined to participate than those who did not. For example, one EP spoke at length about her interest in the inequalities faced by the Somali population which had resulted in her pursuing a professional doctorate in the area. As a result of this, no attempt is made to suggest that the EPs in the current study were a heterogeneously representative sample and that the findings from the current study can be generalised to the wider White, British EP population.

Interestingly, the only two EPs from BME backgrounds, who participated in the Focus Group (Phase One) of the study, withdrew from Phase Two, citing excessive workload and/or time limitations as the reasons why. Through the process of supervision and keeping a reflective diary, I wondered why this waswould these EPs views have differed than those of their White peers? Would 
they have preferred to have discussed their views and experiences of 'race', culture and ethnicity with someone who did not belong to a BME background, as I did? Or, was it that participating in the focus group had highlighted or triggered something that was causing them considerable distress, or emotions they did not want to elaborate on further? Was it that these BME EPs felt more comfortable shunning a research project run by a temporary trainee EP; therefore a 'less threatening' colleague, than engaging in a potentially difficult discussion on a subject matter that is politically charged, highly sensitive and volatile that would risk them being exposed and/or humiliated in front of their long-standing nonBME colleagues?

While it is impossible to provide a definitive link as to reasons why these two EPs declined to participate further in the study, it is acknowledged that this failure to engage and interview these EPs forms a limitation of this study as it would have been valuable to ascertain if they felt their needs had/had not been met with the research at their point of disengagement. By failing to collect further qualitative information through the building up of a rich picture, an opportunity to fully explore the reasons for these two EPs dropping out of the current study was overlooked. Whatever the reasons, their withdrawal from the study in its latter phase highlights the difficult and complex nature of conversations around 'race', culture and ethnicity and indicates that not everybody is comfortable acknowledging theirs, or others, differences.

\subsubsection{Data Collection and Analysis}

Data in the current qualitative study was collected using focus groups and semistructured interviews. Despite being challenging to conduct and time consuming to analyse, the focus groups generated large amounts of qualitative information from discussions in a relatively short period of time and elicited information in a way which allowed me to find out what and why an issue was of particular significance to the EPs. This allowed the gap between what 'EPs say and what they do' to be better understood. The focus groups also allowed, through my presence, integration and further exploration of sensitive information as well as opportunities for me to prompt individuals' to provide breadth and depth to their 
responses. However, as with all research methods, the focus groups had limitations. For example, while it allowed participants to talk to each other, ask questions and express doubts and opinions, I had very little influence over the interaction other than keeping participants focused on the topic. In addition, it should be noted that EPs in the focus groups were speaking in a specific context, within a specific culture and, so, it cannot be always assumed that they were expressing their own definitive individual view(s).

In addition, although not without limitations, the strengths of using focussed semistructured interviews in the present study meant that there was an increased likelihood of positive rapport between myself and the interviewee which made it a very simple, efficient and practical way of getting data about things that could not be easily talked about in the focus groups. The semi-structured interviews allowed flexibility within the interview process which permitted the emergence of unexpected ideas and for the comprehensive exploration of issues unhindered by predefined format.

While data was collected in two phases; thematic analysis was only conducted on the three focus groups to identify final themes and sub-themes. The individual semi-structured interviews were then scrutinised and quotations that supported the sub-themes included. A limitation of this was that new or interesting information that arose from the semi-structured interviews which could not be incorporated into the themes and sub-themes of the focus groups had to be discarded, potentially impacting the findings of the current study. However, as the semi-structured interviews were designed to allow the EPs who had participated in the focus groups to expand and elaborate on their beliefs and experiences and further develop their narratives about 'race', culture and ethnicity, it is felt that they achieved their purpose in generating rich data to support the previously under-researched final themes and sub-themes that emerged from the focus group data. Furthermore, due to the exploratory nature of the current research, it is felt that the final themes and sub-themes derived from the focus group data underwent a comprehensive and thorough check for trustworthiness and therefore provide robust evidence with high validity without the need to over-analyse participants' responses in the semi-structured interviews. 


\subsection{Areas for Further Research}

To enhance the validity of this research, it would be interesting to replicate this study to encompass the voices of EPs in non-urban locations as well as to gain an insight of the impact of 'race', culture and ethnicity on the practise of British BME EPs or other marginalised minority groups, e.g. those with specific sexual, religious affiliations or physical and/or learning disabilities. Moreover, further mixed-methods research should be undertaken with BME CYP-particularly those in pupil referral units (PRUs) or specialist resource provisions- and their families exploring the impact of 'race', culture and ethnicity on their interactions with EPs to determine how comparable the thoughts, feelings and experiences of those on the receiving end are to those of EPs.

In addition, although there is some information on the demography of British EPs (i.e. majority mature and female), there is a lack of research on the linguistic, ethnic and cultural makeup of British EPs overall. This should be explored to determine if there are linguistic, ethnic and cultural gaps among EP personnel that need to be addressed through the recruitment and retention of a diverse range of trainees on EP training courses, in light of the rapidly expanding BME and EAL UK school population.

As highlighted above, EPs specifically recounted the challenges they face working within traded services. As another area that has, as yet, received very little attention in the literature, it would be worth investigating at length the impact of payment on the changing relationship between EPs and their clients. In addition, as also reported by the EPs in this research, further research needs to be undertaken into the area of traded services and ethical working- would funding be spent pragmatically on those most vulnerable, e.g. BME CYP, or prioritised for settings that are more likely to be returning customers based on outcomes? Would EPs compromise their own principles to generate income in an understaffed and struggling service? Although guidelines on ethical working have been published (e.g. AEP, HCPC), there is a need to evaluate traded services to determine how effective they actually are. 


\subsection{Implications for Educational Psychology}

\subsubsection{Services}

As stated above, political and economic changes have led to significant changes in LAs, resulting in the reorganisation of EP services across the UK, which bring with it complexities related to ethical working. As such, there are a number of implications for EP Services:

- Managers within services need to conduct audits and evaluate their services to see if referral systems are further marginalising BME CYPs and their families.

- EPs need to promote on-going supervision between experienced and nonexperienced EPs, specifically in light of the specific issues relating to ethical working as highlighted in this research.

\subsubsection{Schools}

This research indicates that the culture of schools is a significant challenge faced by EPs in their work with those from BME backgrounds:

- EPs need to be involved in training around the psychology of stereotyping, labelling and institutional racism.

- EPs need to recognise these issues when children are referred, e.g. bullying

- EPs need to support opportunities for reflection and supervision so that teachers have greater understanding of their own displaced anger and/or prejudiced attitudes.

- Work that addresses the issues of multicultural competence should be provided to address perceived differences in the values of school staff and CYP and their families.

\subsubsection{Training}

Changes in the training of EPs is one of the central issues this research highlights: 
- EP training courses need to lobby the government on the recruitment of more EPs.

- Course directors need to prioritise recruitment of able and determined individuals from linguistically diverse and multicultural backgrounds to address the needs of the rapidly growing linguistically, culturally and ethnically diverse UK school population.

- Training courses need to ensure that any inequities based on trainees' ethnic or cultural heritage are controlled for and addressed.

- Since dominant forms of ethnicity, i.e. whiteness, become obscure in discussions of multiculturalism (McLaren, 1994), there is need for course directors to support the majority white trainees on courses to undergo opportunities of deconstructing whiteness as a privileged place of racial normativity (Wray and Newitz, 1997, p. 3). This could result in white trainees having open and honest conversations with non-white trainees around 'race', culture and ethnicity, which would, it is hoped, in turn, help BME trainees feel safe in discussing their own experiences.

- Training courses need a curriculum overhaul to include multicultural models, approaches and principles that specifically address the needs of those from BME backgrounds.

\subsubsection{Parents}

Findings from this research indicate that there is a need to engage BME parents in their CYP's education, particularly in relation to SEN and, empower them to access services willingly and independently. This could be achieved through:

- Extended schools, local services and community groups, run by staff sharing the BME parents' ethnicity and/or culture.

- Input from BME professionals in the local area to present vital curriculum topics in a culturally and linguistically sensitive manner.

- Parenting groups or workshops that support BME parents in navigating the educational system and making parents aware of their role in their CYP's education and their legal rights and responsibilities.

- Involving BME parents in groups and networks which link with specific community organisations, e.g. religious or cultural groups and which can be integrated with other mainstream services. 
- Professionals who acknowledge the inherent diversity among BME communities and adapt services appropriately.

- The implementation of culturally specific parenting programmes which reinforce cultural identity and aim to raise parents' confidence in their cultural heritage, which may result in improved attendance on groups run in LAs (Page, Whitting and Mclean, 2007).

- Targeted provision, i.e. availability of interpreters, for parents who speak limited English.

\subsubsection{EPs}

Findings from the current research indicate a shift in perspective of the role of the EP, with an emphasis on understanding and enhancing the quality of life of CYP as well as families and communities. As such, EPs could:

- Get involved in and/or implement prevention/promotion initiatives in the community in which they live.

- Take their knowledge of theoretical frameworks in consultation, learning, conflict resolution, resilience, group processes and apply these to schools and other community groups/organisations to promote positive change.

- Identify needs of communities and where traditional services are failing in meeting the needs of a particular group of people, e.g. the recent influx of refugees, set up self-help groups to address some of issues faced by the community.

- Use their skills of problem solving to help local groups identify issues and empower them through the use of psychological knowledge to address these.

- Engage in participatory research with community groups for the purposes of evaluating the effectiveness of community services and engage in a coordinated way to address any issues and/or concerns.

- Take on advocacy roles for vulnerable groups and communities form decision makers how best their needs can be met. 


\subsection{Researcher Reflexivity}

Qualitative research is surrounded by "interconnected terms, concepts and assumptions" (Denzin and Lincoln, 2000, p. 2) and, as such, there is more than one way of understanding a phenomenon and attaining knowledge (Pope and May, 1999). Therefore, it is perfectly plausible for a researcher's views and personal experiences to be legitimately employed in the interpretation of knowledge. Here, by situating oneself in the participant's world and understanding the personal experiences of the research participants, the researcher can turn these personal experiences into representations that allow interpretation and reveal insights that apply more generally beyond those individuals studied (Lambert, Jomeen and McSherry, 2010). As a result of this, reflexivity, with qualitative research, cannot be underestimated. The process of reflexivity involves the researcher reflecting continuously on their actions, values and perceptions and how these impact upon the research setting as well as how they affect data collection and analysis (Gerrish and Lacey, 2006).

In the current study, as the researcher, I remained cognisant from the early stages of the research of the need to be careful and mindful of the ethnic and social backgrounds of the participants. I felt it was important for me to reflect upon my own identity as a female from a BME background and how this may have been perceived by the majority Caucasian participants. I was also aware that my visible difference may result in socially desirable responses, preventing participants from revealing their true attitudes, opinions and experiences of working with children, young people and families from a BME background. In addition, since the topic being addressed in the current study relates to 'race' and ethnicity- both psychologically and politically sensitive and most-often controversial subjects- and the participants being employed within local authorities, representing diverse populations, I was conscious of the potential effects of impression management (Holgraves, 2004) and participants presenting a favourable image of themselves in front of me and their peers. Throughout the data gathering process, I felt that I needed to guard against the temptation to enlighten the participants of such a perception, whilst also reassuring them that I could empathise with and understand much of their life experiences. In addition, I felt that I would need to be aware of the impact of the 'self' as a researcher as 
this is clearly a crucial aspect of a qualitative research report in terms of the researcher being the instrument employed to collect data, (Ball 1990).

In their guidance on how to conduct appropriate reviews of qualitative research manuscripts, Elliott, Fischer and Rennie (1999) highlight the need for researchers to "...specify their theoretical orientations and personal anticipations, both as known in advance and as they become apparent during the research" (Elliott, Fischer and Rennie, 1999., p. 221). Consequently, a reflective journal was kept throughout the research to record thoughts and feelings that arose in the course of and after interacting with the participants during the focus-group and semistructured interview sessions as well as during the data analysis and write-up phases. The purpose of this was to enable me to undertake reflection-on-action and reflection-in-action (Schon, 1983) and to expose myself to numerous thoughts, feelings and experiences of the EPs during the focus groups and semistructured interviews.

As noted earlier, this research was initially undertaken as a therapeutic means of dealing with the emotional maelstrom and professed ineptitude I experienced during my first year as a trainee EP. I hoped that sharing my experiences with other EPs would give me an individualised and definitive insight into whether or not I would have to compromise my identity to practise as an EP by conforming to the majority views or, if I should instead carry my BME identity with poise and aplomb in an attempt to counteract and/or neutralise the educational inequalities faced by others from BME communities. Through the reflective process, what I have found, instead, is that I have not only become more conscious of my own thoughts, feelings and experiences but, also feel privileged to have been exposed to the thoughts, feelings and experiences of the EPs who participated in this research. I found the process of engaging in open and honest conversations, which every so often left both parties feeling emotionally vulnerable, led to distinct and progressive interpretations and meanings which had, thus far, remained unexplored.

I also gained a greater understanding of the complexity of issues around 'race', culture and ethnicity, in that, I somewhat empathised with the EPs in terms of their work with those from BME backgrounds. I noted that, like the cultural 
paradox I experienced, i.e. loathing being labelled or stereotyped by others versus being open to the idea of being asked questions about my 'racial', cultural and ethnic heritage when faced with discriminatory attitudes, comments and behaviours from others, EPs too, faced a similar paradox, i.e. struggling to understand or relate to unfamiliar customs, behaviours or practices yet due to the politicisation of diversity and multiculturalism, e.g. Brexit, and their fear of offending; generalising customs, behaviours or practices across all/similar cultures instead.

Due to the sensitive nature of the topic under study and the feelings it had aroused in me, I felt it important to ask for feedback from the EPs following their participation in the research. I felt somewhat uncomfortable about having personally gained from this process and not knowing if participating in the study had been worthwhile, or not, for the EPs. I felt relieved when several EPs reported feeling positive about participating in the current study and stating that the discussions that had taken place within the focus groups, had continued beyond the study:

KP: $\quad$...it was quite interesting, I think it was, a topic that's obviously very relevant for all EPs and although you know we went along because it was for your research, actually when we started talking about it, it, you know I, I realised that actually, I was learning things about sort of ethical issues that are relevant for us in, you know, whether it was your research or not... and we all have an opinion on it, don't we? And, we all have a, you know, something that really needs to be discussed...frequently, really! Because it's something I don't think we've ever really discussed- I don't remember, I mean there's always an ethical aspect to whatever we do, , and I think working in [borough] because it's such a... a range of different, pupils, different backgrounds for different religion and culture... it's just something we accept (9-19). 


\section{References}

Abbott, D. (MP). (2010). The aftermath of getting it right. In Weekes-Bernard, D. (Ed.). Did they get it right? A re-examination of school exclusions and race equality. (pp.10-11). London: Runnymede Trust.

Abedi, J., Hofstetter, C., and Lord, C. (2001). NAEP math performance and test accommodations: Interactions with student language background. Educational and Child Psychology, 10 (3), 115-130.

Abelson, R. P., Dasgupta, N., Park, J., and Banaji, M. R. (1998). Perceptions of the collective other. Personality and Social Psychology Review, 2, 243-250.

Agyemang, C., Bhopal, R., and Bruijnzeels, M. (2005). Negro, Black, Black African, African Caribbean, African American or what? Labeling African origin populations in the health arena in the 21st century. Journal of Epidemiology \& Community Health, 59(12), 1014-1018.

Ajegbo, K., Kiwan, D. and Sharma, S. (2007). Curriculum review: Diversity and Citizenship. London: DfES.

American Anthropological Association www.aaanet.org/issues/policyadvocacy/AAA-Statement-on-Race.cfm.

(1998),

American Psychological Association, (1993). Guidelines for providers of psychological services to ethnic, linguistic and culturally diverse populations. American Psychologists, 48, 45-48.

American Psychological Association, (2003). Guidelines on Multicultural Education, Training, Research, Practice, and Organisational Change for Psychologists. $\quad$ http://www.apa.org/pi/oema/resources/policy/multiculturalguideline.pdf

Anderson, R. (1999). Impact of First Language Loss on Grammar in a Bilingual Child. Communication Disorders Quarterly, 21: 4-16. 
Anderson. H. and Goolishian. H. (1988). Human systems as linguistic systems: Preliminary and evolving ideas about the implications for clinical theory. Family Process. 27, 157-163.

Aronson, J. (1994). A pragmatic view of thematic analysis. The Qualitative Report, 2(1), 15-19.

Arredondo, P. (1999). Multicultural counseling competencies as tools to address oppression and racism. Journal of Counseling \& Development, 77, 102-108.

Ashworth, P.D. (2003). The origins of qualitative psychology. In J.A. Smith (ED.). Qualitative psychology: A practical guide to research methods. London: Sage

Association of Educational psychologists (2011). The Delivery of Educational psychology services. Durham: AEP

Attride-Stirling, J. (2001). Thematic networks: an analytic tool for qualitative research. Qualitative Research, 1(3), 385-405.

Austen-Smith, D. and Fryer R. G., Jr. (2005). An Economic Analysis of 'Acting White,' Quarterly Journal of Economics, 1(2), 551-583.

Bainter, T. R. and Tollefson, N. (2003). Intellectual assessment of language minority students: What do school psychologists believe are acceptable practices? Psychology in the Schools, 40, 599-603. DOI: 10.1 002/pits.I 0131

Baker, C. (2008). Foundations of bilingual education and bilingualism. Clevedon, England: Multilingual Matters.

Ball, S.J. (1990). Self-doubt and soft data: social and technical trajectories in ethnographic field work. International Journal of Qualitative Research, 3(2), 157-71. 
Banister, P., Burman, E., Parker, I., Taylor, M. and Tindall, C. (1994). Qualitative Methods in Psychology: A Research Guide. Buckingham: Open University Press.

Barber, B. and Olsen, J. (2004). Assessing the transitions to middle and high school. Journal of Adolescent Research, 19. 4-23.

Barkan, E., S. (2012). Social Problems: Continuity and Change. New York: Flat World Knowledge.

Beck, A. T. (1979). Cognitive therapy of depression. New York: Guilford Press.

Bedore, L.M. and Pena, E.D. (2008) Conceptual versus Monolingual Scoring: When does it make a difference? Speech, Language, Hearing Services in Schools, 36, 188-200.

Bennett, L., Jr. (1988). Before the Mayflower: A History of Black America. New York: Penguin Books

Bernal, M. (1987). Black Athena: The Afrosaic Roots of Classical Civilization. New Brunswick, NY: Rutgers University Press.

Bhopal R (2004). Glossary of terms relating to ethnicity and race: for reflection and debate. Journal of Epidemiology and Community Health On-Line, 58, 4415.

Bialystok E. (2001). Metalinguistic aspects of bilingual processing. Annual Review of Applied Linguistics, 21, 169-181. 
Bitter Davis, G. and Parker, C. A. (1997). Writing the doctoral dissertation: a systematic approach (2 ${ }^{\text {nd }}$ Edn.), London: Barron's Educational Series.

Bogdan, R.C., and Biklin S.K. (1998). Qualitative research for education: An introduction to theory and methods. (3rd Ed.). Boston: Allyn and Bacon.

Bonilla-Silva, E. (2009). Racism without racists: Colorblind racism and the persistence of racial inequality in the United States (3rd Ed.). Lanham, MD: Rowman and Littlefield.

Booth, A. L., Leigh, A. and Varganova, E. (2012). Does ethnic discrimination vary across minority groups? Evidence from a field experiment. Oxford Bulletin of Economics and Statistics, 74(4), 547-573. DOI: 10.1111/j.1468$\underline{0084.2011 .00664 . x}$

Bowling, B. and Phillips, C. (2003). Racist victimization in England and Wales. In: Hawkins, Darnell F., (ed.) Violent Crime: Assessing Race and Ethnic Differences. Cambridge studies in criminology (pp. 154-170). Cambridge: Cambridge University Press,

Boyatzis, R. E. (1998). Transforming qualitative information: Thematic analysis and code development. Thousand Oaks, CA: Sage.

Bracken, S. (2010). Discussing the importance of ontology and epistemology awareness in practitioner research. Worcester Journal of Learning and Teaching, Issue 4, pp. 1-9

Braun, V. and Clarke, V. (2006) Using thematic analysis in psychology. Qualitative Research in Psychology, 3 (2). 77-101. Available from: http://eprints.uwe.ac.uk/11735

British Psychological Society (2009). Code of ethics and conduct. Leicester: BPS. 
Bronfenbrenner, U. (1979). The ecology of human development: Experiments by nature and design. Cambridge, MA: Harvard University Press.

Broomfield, J., and Dodd, B. (2004). Children with speech and language disability: Caseload characteristics. International Journal of Language \& Communication Disorders, 39(3), 303-324.

Bryman, A. (1988). Quantity and Quality in Social Research. London: Unwin Hyman

Burden, R. (1997). Research in the real world. An evaluation model for use by applied educational psychologists. Educational Psychology in Practice, 13 (10), 13-20.

Burr, V. (1995). An introduction to social constructionism. London: Routledge.

Byrne-Armstrong, H., Higgs, J. and Horsfall, D. (2001). Critical Moments in Qualitative Research. London: Elsevier Health Sciences.

Canino, I. A., and Spurlock, J. (2000). Culturally diverse children and adolescents: Assessment, diagnosis and treatment. New York: Guilford Press.

Chant, S. (2002) Whose Crisis? Public and Popular Reactions to Family Change in Costa Rica. In Christopher Abel and Colin Lewis (Editors) Exclusion and Engagement: Social Policy in Latin America (pp. 349-377). London: Sage

Clark, C. R., Mercer, S. H., Zeigler-Hill, V. and Dufrene, B. A. (2012). Barriers to the Success of Ethnic Minority Students in School Psychology Graduate Programs. School Psychology Review, 41(2), 176-192.

Cline, T. and Frederickson, N. (2009) Special Educational Needs, Inclusion and Diversity: A Textbook. Second Edition. Buckingham: Open University Press 
Cline, T. and Shamsi, T. (2000) Language needs or special needs? The assessment of learning difficulties in literacy among children learning English as an additional language: a literature review [RR 184]. London: DfES.

Cline, T., Abreu, G. de, Fihosy, C., Gray, H., Lambert, H., and Neale, J. (2002). Minority Ethnic Pupils in Mainly White Schools. London: Department for Education and Skills.

Cohen, L., Manion, L. and Morrison, K. (2000). Research Methods in Education (5th Edn.). London: Routledge

Comas- Diaz, L. (1998). Ethnic Minority Psychology: Identity, Empowerment and Transformation. Cultural Diversity and Mental Health, 4 (3), 151-152.

Commission for Racial Equality. (2007). Ethnic Minorities in Britain Fact file 2

Constantine, M. G., and Ladany, N. (2000). Self-report multicultural counselling competence scales: Their relation to social desirability attitudes and multicultural case conceptualization ability. Journal of Counselling Psychology, 47, 155-164.

Coolican, H. (2004). Research methods and statistics in psychology. London: Hodder \& Stoughton.

Cording, J. (2011). A study of Educational Psychologists' use of consultation and users' views on what a service should deliver. Retrieved from https://ore.exeter.ac.uk/repository/bitstream/handle/10036/3294/CordingJ.pdf ?sequence=1

Crutchley, A. (1999). Bilingual children with SLI attending language units: getting the bigger picture. Child Language Teaching and Therapy, 201-217. 
Curtis, M. J., Grier, J. E. C. and Hunley, S. A. (2004). The changing face of school psychology: Trends in data and projections for the future. School Psychology Review, 33, 49-66.

Curtis, M. J., Lopez, A. D., Castillo, J. M., Batsche, G. M., Minch, D. and Smith, J. C. (2008). The status of school psychology: Demographic characteristics, employment conditions, professional practices, and continuing professional development. Communiqué, 36, 27-29.

Curtis, M.J., Castillo, J. M. and Gelley, C. (2012). School psychology 2010: Demographics, employment, and the context for professional practice-Part I. Communiqué, 40(7), 1; 28-30.

Curtis, M.J., Walker, K.J., Hunley, S.A., and Baker, A.C. (1999). Demographic characteristics and professional practices in school psychology. School Psychology Review, 28, 104-116.

D'Angelo, A., Paniagua, A. and Ozdemir, A. (2011). BME Children in London: Educational Needs and the Role of Community Organisations (An evaluation of the education services of Day-Mer, Turkish and Kurdish Community Centre). Middlesex University, London.

D'Ardenne, P., and Mahatani, A. (1999). Transcultural Counselling in Action (Second Ed.). London: Sage.

Darlaston-Jones, D. (2007). Making connections: The relationship between epistemology and research methods. The Australian Community Psychologist, 19(1), 19-27.

David, M. and Sutton, C., (2004). Social research: the basics. Thousand Oaks, CA: Sage.

Davis, A., Mclntosh, D., Phelps, L. and Kehle, T. (2004). Addressing the shortage of school psychologists: A summative overview. Psychology in the Schools, 41, 489 - 495. DOI: 10.1002/pits.10192 
Dearing, R. (1996) Review of qualifications for 16-19 year olds [Full Report]. London: School Curriculum and Assessment Authority.

Department for Education (2010). The Academies Act. London: DfE

Department for Education (2012). A profile of pupil exclusions in England Research Report. Education Standards Analysis and Research Division. London: HMSO

Department for Education (2014). Children and Families Act. London: DfE.

Department for Education and Science (1988). The Education Reform Act. London: HMSO.

Department for Education and Skills (2004). The Children's Act. Every Child Matters: Change for Children. London: HMSO.

Departments for Education and Health (2015). Special Educational Needs and Disability Code of Practice: 0 to 25 years. London: DfE and DoH

Dervin, B. (1983). An overview of sense-making research: Concepts, methods and results. Paper presented at the annual meeting of the International Communication Association, Dallas, TX, May. [On-line]. Available: http://communication.sbs.ohio-state.edu/sense-making/art/artdervin83.html

Deutsch, R. and Reynolds, Y. (2000). The use of dynamic assessment by educational psychologists in the UK, Educational Psychology in Practice, 16(3), 311-331 
Dickerson, V. C. and Zimmerman, J. L. (1996). Myths, misconceptions, and a word or two about politics (Special edition on narrative, J. L. Zimmerman \& V. C. Dickerson, Eds.). Journal of Systemic Therapies.

Division of Education and Child Psychology (2002). Inclusive Education Position Paper. Accessed on 25/11/2012 from http://decp.bps.org.uk/decp/position statements/position statements home.cf $\underline{\mathrm{m}}$

Division of Educational and Child Psychology (2010). Inclusive Education Position Paper, London: British Psychological Society

Duncan, D. M., 1989, Theory and practice. In D. Duncan (ed.), Working with Bilingual Language Disability. London: Chapman \& Hall, pp. 3-17.

Duncan, D. M., 1989, Theory and practice. In D. Duncan (ed.), Working with Bilingual Language Disability. London: Chapman and Hall, pp. 3-17.

Dunn, K (1987). Teaching students through their individual learning styles: A practical approach. Reston, VA: Reston Publishing Company

Dunn, L. M., Dunn, L. M., Whetton, C., \& Pintilie, D. (1982). British Picture Vocabulary Scale. Windsor: NFER-Nelson.

Dunn, L., Padilla, E., Lugo, D., \& Dunn, L. (1986). Test de Vocabulario en Imágenes Peabody -- Adaptación Hispanoamericana [Peabody Picture Vocabulary Test -- Latin American adaptation]. Circle Pines, MN: American Guidance Service.

Duran, R.P. (1989). Assessment and instruction of at- risk Hispanic students. Exceptional Children, 56 (2), 154-158. 
Durrheim, K., Hook, D., and Riggs, D. W. (2009). Race and Racism. In D. Fox, I. Prilleltensky, and S. Austin (Eds.) Critical Psychology: An Introduction (Second Ed.) (pp. 197-214). Washington, DC: SAGE

Echevarria, J., Short, D., and Powers, K. (2006). School reform and standardsbased education: How do teachers help English language learners? Journal of Educational Research. 2(2). 12-17.

Edwards, P. (1967). The Encyclopaedia of Philosophy, Vol. 5. New York: Macmillan.

Elliott, B., (2000). Commitment to teaching: Australian perspectives on the interplays of the professional and the personal in teachers' lives. Educational Review, 33(3), 181-190.

Elliott, R., Fischer, C.T. and Rennie D.L. (1999). Evolving guidelines for publication of qualitative research studies in psychology and related fields. British Journal of Clinical Psychology, 38, 215-229

Esterberg, K. G. (2002). Qualitative methods in social research. Boston: McGraw-Hill.

Fagan, T. K. (1988). The historical improvement of the school psychology service ratio: Implications for future employment. School Psychology Review, $17,447-458$

Fagan, T. K. and Wise, P. S. (2000). School psychology: Past, present, and future (2nd Ed.). Bethesda, MD: National Association of School Psychologists.

Felson, R. B., Liska, A. E., South, S. J., and McNulty, T. L. (1994). The subculture of violence and delinquency: Individual vs. school context affects. Social Forces, 73(1), 155-173.

Fernando, S. (2010). Mental Health, Race and Culture (Third Edition). London: Palgrave Macmillan 
Ferraro, G. (1998). The Culture Dimensions of International Business, London: Prentice-Hall

Fiegener, M.K. (2009). Doctorate recipients from U.S. universities: Summary report 2007- 2008. Arlington, VA: National Science Foundation.

Figueroa, R. A. (2002). Issues in testing students from culturally and linguistically diverse backgrounds. Multicultural Education. 2 (1), 10-19.

Figueroa, R. A., and Newsome, P. (2006). The diagnosis of LD in English learners: Is it non-discriminatory? Journal of Learning Disabilities, 39, 206-214

Finlay, L. (2003a) The reflexive journey: mapping multiple routes, in: L. Finlay and B. Gough, Reflexivity: a practical guide for researchers in health and social sciences, (pp. 3-20). Oxford: Blackwell Science.

Florio-Ruane, S. (1991). Conversation and narrative in collaborative research. In C. Witherell and N. Noddings (Eds.). Stories lives tell: narrative and dialogue in education, (pp. 234-256). New York: Teachers College Press.

Flyvbjerg, B. (2006). Five Misunderstandings About Case-Study Research. Qualitative Inquiry, 12(2), 219-245. DOI: $\underline{10.1177 / 1077800405284363}$

Fritzberg, G. (2001). Less than equal: A former urban schoolteacher examines the causes of educational disadvantagement. The Urban Review, 33. 109-119.

Fueyo, V., and, Bechtol, S. (1999). Those who can teach: Reflecting on teaching diverse populations. Teacher Education Quarterly, 26, 1-10.

Gaine, C. (2005). We're All White Thanks. Stoke on Trent: Trenham Books. 
Gallagher, K. (2008). The Methodological Dilemma: Creative, critical, collaborative and post-positivist. London: Rutledge.

Genesee, F. (2004). Second language learning through immersion: A review of U. S. programs. Review of educational research, 55 (4), 541-561.

Gergen, K. and Gergen, M. (1991). Toward Reflexive Methodologies. In Steier, Frederick (Ed.), Research and Reflexivity, London: Sage.

Gergen, K. J. (1999). An Invitation to Social Construction. London: Sage

Gerrish, K. and Lacey, A. (2006). The Research Process in Nursing (5th Edn). Oxford: Blackwell Publishing.

Gillborn, D. (1997). 'Young, black and failed by school', International Journal of Inclusive Education, 1(1): 1-23.

Gillborn, D. (2008). Coincidence or conspiracy? Whiteness, policy and the persistence of the Black/White achievement gap. Educational Review, 60 (3). pp. 229-248.

Gillborn, D. and Gipps, C. (1996). Recent Research on the Achievements of Ethnic Minority Pupils. London, London: HMSO.

Gitlin, T. (1990). Who communicates what to whom, in what voice and why: about the study of mass communication. Critical Studies in Mass Communication, 7(2), 185-196. 
Glaser, B. and Strauss, A. (1967). Discovery of Grounded Theory. Chicago: Aldine.

Glesne, C. and Peshkin, A. (1992). Becoming qualitative researchers: An introduction. White Plains, NY: Longman.

Goh, D.S., Teslow, C.J., and Fuller, G.B. (1981). The practice of psychological assessment among school psychologists. Professional Psychology, 72, 696706

Goldstein, C.N., (2004). Local knowledge, research knowledge, and educational change: A case study of early Spanish reading improvement. Educational Researcher 20, 2-14.

Gollnick D. M., and, Chinn, P. C. (1991). Multicultural education for exceptional children. ERIC Digest \# 498. Retrieved June 22, 2016 from http://www.ed.gov/databases/ERIC Digests/ed333620.html

Gonzalez, R. C., Biever, J. L. and Gardner, G. T. (1994). The multicultural perspective in psychotherapy: A social constructionist approach. Psychotherapy: Theory, Research, Practice, Training, 31(3), 515-524.

Gopaul-McNicol, S. and Thomas-Presswood, T. (1998). Working with linguistically and culturally different children: Innovative clinical and educational approaches. Boston: Allyn and Bacon.

Gossett, T., F. (1963). Race: The History of an Idea. Dallas, TX: Southern University Press.

Graue, E and Walsh, J. (1998). Studying children in context: theories, methods, and ethics. London: Sage.

Gravelle, M., (1996). Supporting Bilingual Learners in Schools. UK: Trentham Books 
Greene, J. P., and Winters, M. A. (2005). Public high school graduation and college-readiness rates: 1991-2002. Manhattan Institute Education Working Paper No.8. Retrieved electronically from: http://www.manhattaninstitute.org/html/ewp_08.htm on February 2, 2006.

Guba, E. and Y. Lincoln (1989) Fourth Generation Evaluation. Newbury Park, CA: Sage.

Guba, E.G. (1981). Criteria for assessing the trustworthiness of naturalistic inquiries. Educational Communication and Technology Journal, 29 (2), 75-91.

Guess, J. (2006). The Social Construction of Whiteness: Racism by Intent, Racism by Consequence. Critical Sociology, 32: 649-673. DOI: $\underline{10.1163 / 156916306779155199}$

Guest, G., MacQueen, K. M. and Namey, E. E. (2012). Applied thematic analysis. Thousand Oaks, CA: Sage

Gutierrez-Clellen, V. (1999). Language choice in intervention with bilingual children. American Journal of Speech-Language Pathology, 8, 291-302.

Gutierrez-Clellen, V. and Pena, E. (2001). Dynamic assessment of diverse children: A tutorial. Language, Speech and Hearing Services in Schools, 32, 212-224.

Hall, B. C. (1995). Attitudes of fourth and sixth graders toward peers with mild articulation disorders. Language, Speech, and Hearing Services in Schools, 22(1), 334-340

Hamnett, C. and Butler, T. (2011), 'Geography matters': the role distance plays in reproducing educational inequality in East London. Transactions of the Institute of British Geographers, 36: 479-500. DOI: 10.1111/j.1475$\underline{5661.2011 .00444 . x}$ 
Harrell, M.C. and Bradley, M.A. (2009). Data Collection Methods: SemiStructured Interview and Focus Groups. Santa Monica: National Defense Research Institute.

Haywood, H.C. and Lidz, C.S. (2007). International Survey of Dynamic Assessment Trainers. Journal of Cognitive Education and Psychology, 5(2), 181-198.

Heitritter, D.L. (1999). Somali Family Strength: Working in the Communities. A Report from Meanings of Family Strength Voiced by Somali Immigrants: Reaching an Inductive Understanding. Family Social Science Department, University of Minnesota.

Held, B. (1990). What's In A Name? Some conclusions and concerns about constructivism, Journal of Marital and Family Therapy, 16 (2), 179-186.

Henning-Stout, M. and Brown-Cheatham, M. (1999). School psychology in a diverse world: Considerations for practice, research, and training. In C. R. Reynolds \& T. B. Gutkin Eds.), The handbook of school psychology (3rd Ed.). (pp. 1041-1055). New York: Wiley and Sons

Hofstede, G. (1991). Cultures and Organizations: Software of the Mind. London, UK: McGraw-Hill.

Holgraves, T. (2004). Social Desirability and Self-Reports: Testing Models of Socially Desirable Responding. Personality and Social Psychology Bulletin, 30, 161-172.

Home Office (1999) Stephen Lawrence Inquiry: the Home Secretary's Action Plan. London: Home Office [police.homeoffice.gov.uk/publications/]

Hosp, J. L. and Reschly, D. J. (2002). Regional differences in school psychology practice. School Psychology Review, 31, 11-29. 
Howe, K. R. (1988). Against the quantitative-qualitative incompatibility thesis, or, Dogmas die hard. Educational Researcher, 17, 10-16.

Huebner, E. S. (1993). Psychologists in secondary schools in the 1990's: current functions, training, and job satisfaction. School Psychology Quarterly, $8,50-56$.

Hunter, M. (2002). Rethinking epistemology, methodology, and racism: or, is White sociology really dead? Race and Society, 5, 119-138

Hutchinson, G., and McKenzie, K. (1995): What is an Afro-Caribbean? Implications for psychiatric research. Psychiatric Bulletin, 19:700-702. DOI: $\underline{10.1192 / p b .19 .11 .700}$

Ingraham, C. L. (2000). Consultation through a multicultural lens: Multicultural and cross-cultural consultation in schools. School Psychology Review, 29, 320343.

Ivey, A. (1995). Psychotherapy as liberation: Toward specific skills and strategies in multicultural counselling and therapy. In J. Ponterotto, J. Casas, L. Suzuki and C. Alexander (Eds.), Handbook of multicultural counselling (pp. 53-72). Thousand Oaks, CA: Sage.

James, D. G. H. (1995). The assessment and management of children from a non-English speaking background who have language problems. Australian Journal of Human Communication Disorders, 23, 72-88.

Jenkins, R. (1986). Social Anthropological Models of Inter-Ethnic Relations. In J. Rex and D. Mason (Eds.), Theories of Race and Ethnic Relations (pp. 170186). Cambridge: Cambridge University Press.

Joffe, H. (2011). Thematic analysis. In D. Harper \& A. R. Thompson (Eds), Qualitative methods in mental health and psychotherapy: A guide for students and practitioners, (pp. 209-224). Chichester: Wiley. 
Joffe, H. and Yardley, L. (2004). Content and thematic analysis. In D. F. Marks \& L. Yardley (Eds), Research methods for clinical and health psychology, (pp. 56-68). London: Sage.

Johnson, R. B. and Christensen, L. B. (2010). Educational research: Quantitative, qualitative, and mixed approaches (4th Ed.). Thousand Oaks, CA: Sage.

Jonassen, D. H. (1991). Objectivism versus constructivism: do we need a new philosophical paradigm? Journal of Educational Research, 39 (3), 5-14.

Jung, E. A. (23/07/2015). When Jon Stewart Was Challenged About Race. Vulture (Online). http://www.vulture.com/2015/07/when-jon-stewart-waschallenged-about-race.html

Kahin, M (1997). Educating Somali Children in Britain. Stoke on Trent: Trentham Books.

Katz, J. (1985). The socio-political nature of counselling. The Counselling Psychologist, 13, 615-624.

Kitzinger, J. (1994). The methodology of focus groups: The importance of interaction between research participants. Sociology of Health and IIIness, 16(1), 103-121.

Kreuger, A., R., and Casey, A., M. (2000). Focus Groups. A Practical Guide for Applied Research (3rd Edition). Thousand Oaks, CA: Sage Publications

Ladany, N., Inman, A. G., Constantine, M. G., and Hofheinz, E. W. (1997). Supervisee multicultural case conceptualization ability and self-reported multicultural competence as functions of supervisee racial identity and supervisor focus. Journal of Counseling Psychology, 44, 284-293. 
Ladson-Billings, G. (1994). The dream keepers: Successful teachers of African American children. San Francisco: Jossey-Bass.

Lambert, C., Jomeen, J. and McSherry, W. (2010). Reflexivity: a review of the literature in the context of midwifery research. British Journal of Midwifery, 18 (5), 321-326

Lankshear A.J. (1993). The use of focus groups in a study of attitudes to student nurse assessment. Journal of Advanced Nursing 18: 186-189.

Larney, R. (2003). School-Based Consultation in the United Kingdom: Principles, Practice and Effectiveness. School Psychology International, 24(1), 5-19.

Litosseliti, L. (2003). Using focus groups as research. London: Continuum.

Little, S. and Garber, J. (2004). Interpersonal achievement orientations and specific stressors predict depressive and aggressive symptoms. Journal of Adolescent Research, 19. 64-73.

Lopez, E. C. and Rogers, M. R. (2001). Conceptualizing cross-cultural school psychology competencies. School Psychology Review, 16, 270-302. Retrieved from http:// www.nasponline.org/publications/spr/sprmain.as px

Lucchino P., Rosazza-Bondibene, C., and Portes, J. (2012). Examining the Relationship between Immigration and Unemployment using National Insurance Number Registration Data, National Institute of Economic and Social Research Discussion Paper No.286.

Lynch, K. and Baker, J. (2005). Equality in education: An equality of condition perspective. Theory and Research in Education, 3(2), 131-164. DOI: $\underline{10.1177 / 1477878505053298}$ 
Machery, E. and Faucher, L. (2005). Social Construction and the Concept of Race. Philosophy of Science, 3(4), 1208-1219.

Marshall, C., and, Rossman G.B. (1999). Designing qualitative research (3rd Ed.). Newbury Park: Sage.

Maxwell, J. (1997). Designing a qualitative study. In L. Bickman and D. J. Rog (Eds.). Handbook of applied social research methods (pp. 69-100). Thousand Oaks, CA: Sage.

McCloskey, D. and Athanasiou, M. (2000). Assessment and intervention practices with second language learners among school psychologists. Psychology in Schools, 37,209- 225.

McLaren, P. (1997). Decentering whiteness. Multicultural Education, 5(1), 411.

Mcleod, J. (1994). Doing counselling research. London: Sage

Mehmetoglu, M. and Altinay, L. (2006). Examination of Grounded Theory Analysis with an Application to Hospitality Research. International Journal of Hospitality Management, 25(1), 12-33.

Mennen, I., Stansfield, J. and Johnston, S. (2005). Speech and language therapy services for bilingual children in England and Scotland: a tale of three cities. International Symposium on Bilingualism, 12, 1578-1596.

Merriam, S. B., Caffarella, R. S. and Baumgartner, L. (2007). Learning in adulthood: A comprehensive guide (3rd Ed.). San Francisco: Jossey-Bass.

Mertens, D. M. (1998). Research methods in education and Psychology: Integrating diversity with quantitative and qualitative approaches. London: Sage. 
Merton, R. K. (1975). Thematic analysis in science: notes on Holton's concept. Science as Culture, 188, 335-338.

Mewborn, D. S. (2005, October). Framing our work. In G. M. Lloyd, M. Wilson, J. L. M. Wilkins, \& S. L. Behm (Eds.), Proceedings of the 27th annual meeting of the North American Chapter of the International Group for the Psychology of Mathematics Education, Roanoke, VA. Retrieved from http://www.pmena.org/proceedings/PMENA $\% 2027 \% 202005 \% 20$ Proceedings. pdf

Mewborn, K. N. (2001). Multicultural counselling competencies: An exploratory investigation of multicultural training in school psychology. Unpublished master's thesis, University of Maryland, College Park.

Miles, M. B. and Huberman, A. M. (1994). Qualitative data analysis: An expanded sourcebook. Thousand Oaks, CA: Sage.

Miles, M. B., Huberman, A. M. and Saldana, J. (2014). Qualitative Data Analysis: A Methods Sourcebook and The Coding Manual for Qualitative Researchers. Thousand Oaks, CA: SAGE, 2014.

Miller, D. C. and Palamores, R. (2000). Growth in school psychology: A necessary blueprint. Communiqué, 28(6), 6-7.

Miranda, A. H. (2002). Best practices in increasing cross-cultural competence. In A. Thomas and J. Grimes (Eds.), Best Practices in School Psychology, Fourth Ed. Washington, DC: National Association of School Psychologists.

Morewood, G. D. and Rumble, A. (2013). Developing a 'local offer': A new Trainee Educational Psychologist model. Assessment and Development Matters, 5(1):33-35.

Morgan, D. L. (1988). Focus groups as qualitative research. Newbury Park, CA: Sage. 
Naidoo, A.V. (1996). Challenging the hegemony of Eurocentric psychology. Journal of Community and Health Sciences, 2(2), 9-16.

National Association of School Psychologists (2000). Standards for training and field placement programs in school psychology. Bethesda, MD. http://nasponline.org/multicultural

National Association of School Psychologists. (2008). [Fluency in and use of languages other than English among NASP members.] Unpublished data from the 2004-05 NASP Membership Survey

National Centre for Educational Statistics, (2012). The Condition of Education 2012. U.S. Department of Education.

National Collage for Teaching and Leadership (2016). Review of clinical and educational psychology training arrangements, Department for Education, London. Downloaded from: https://www.gov.uk/government/uploads/system/uploads/attachment data/file/ 510725/Review of clinical and educational psychology training arrangeme nts report.pdf

Nayak, H., Hansen, N., Krueger, N., and McLaughlin, B. (1990). Languagelearning strategies in monolingual and multilingual adults. Language Learning, 40, 221-244.

Nicoladis, E. and Genesee, F. (1996). A longitudinal study of pragmatic differentiation in young bilingual children. Language Learning, 46, 439-464.

Nieto, S., and Bode, P. (2008). Affirming diversity (5th Ed.). Boston: Allyn and Bacon

Nunan, D. 1992. Research methods in language learning. Cambridge: Cambridge University Press. 
O'Bryon, E. C. and Rogers, M. R. (2010). Bilingual school psychologists' assessment practices with English language learners. Psychology in the Schools, 47, 1018-1034. DOI: 10.1002/pits.20521

Ochoa, S. H., Rivera, B., and Ford, L. (1997). An investigation of school psychology training pertaining to bilingual psycho-educational assessment of primarily Hispanic students: Twenty-five years after Diana v. California. Journal of School Psychology, 35,329-349

Office for National Statistics; National Records of Scotland; Northern Ireland Statistics and Research Agency (2016): 2011 Census aggregate data. UK Data Service (Edition: June 2016). DOI: http://dx.doi.org/10.5257/census/aggregate$\underline{2011-1}$

Oller, K.D., and Eilers, R. E.(Eds.) (2002). Language and literacy in bilingual children. New York: Multilingual Matters.

Omi, M. and Winant, H. (1994). Racial formation in the United States. New York: Routledge.

Orfield, G. (Ed.) (2001). Diversity challenged: Evidence on the impact of affirmative action. Cambridge, MA: Harvard Educational Publishing Group.

Ortiz, S., and Flanagan, D. (2002). Best practices in working with culturally different families. In A. Thomas and J. Grimes (Eds.), Best Practices in School Psychology, Fourth Edition (pp. 337-351). Washington, DC: National Association of School Psychologists.

Owen, I. R. (1992). Applying social constructionism to psychotherapy. Counselling Psychology Quarterly, 5(4), 385-402

Paradis, J. and Genesee, F. (1996). Syntactic acquisition in bilingual children: Autonomous or Interdependent? Studies in Second Language Acquisition, 18, 1-25. 
Patton, M. (2002). Qualitative research \& evaluation methods (3 ${ }^{\text {rd }}$ ed.). Thousand Oaks, California: SAGE.

Patton, M. Q. (1990). Qualitative research and evaluation methods (2nd Ed.). Newbury Park, CA: Sage.

Peal. E., and Lambert, M. (1962). The relation of bilingualism to intelligence. Psychological Monogruph, 76(546). 1-23.

Pedersen, P. (1999). Multiculturalism as a Fourth Force. Philadelphia, PA: Bruner/Maze

Pedersen, P. B. (2002). The Making of a Culturally Competent Counselor. Online Readings in Psychology and Culture, 10(3).

Pehrson, S., Vignoles, V. L. and Brown, R. (2009). National identification and anti-immigrant prejudice: Individual and contextual effects of national definitions. Social Psychology Quarterly, 72(1), 24-38.

Perry, W. G. (1970). Forms of intellectual and ethical development in the college years: A scheme. New York: Holt, Rinehart and Winston.

Pitts, J.M. (1994). Personal understandings and mental models of information: A qualitative study of factors associated with the information-seeking and use of adolescents. Ph.D. dissertation. Florida State University.

Poehner, M. E., and Lantolf, J. P. (2005). Dynamic assessment in the language classroom. Language Teaching Research, 9, 233-265.

Poland, B. (2002). Transcription quality. In Gubrium, J.F. and Holstein, J.A. (Eds.) Handbook of Interview Research. Thousand Oaks, Sage. Reprinted in 
Holstein, J.A. and Gubrium, J.F. (Eds). (2003). Inside Interviewing: New Lenses, New Concerns. Thousand Oaks, CA, Sage.

Pope, C. and May, N. (1999). Qualitative Research in Health Care (2nd Edn.). London: Blackwell Publishing.

Powell, R.A. and Single, H.M. (1996). Focus groups. International Journal of Quality in Health Care, 8 (5), 499-504.

Primeau, A. L. (2003). Reflections on Self in Qualitative Research: Stories of Family. American Journal of Occupational Therapy, 57, 9-16. DOI: 10.5014/ajot.57.1.9

Pring, R. (2000a). Philosophy of Educational Research, London: Continuum.

Quinn, C., 2001, The developmental acquisition of English grammar as an additional language. International Journal of Language and Communication Disorders, 36, 309-314.

Reichardt, C. S., and Cook, T. D. (1979). Beyond qualitative versus quantitative methods. In T. D. Cook and C. S. Reichardt (Eds.). Qualitative and quantitative methods in evaluation research. Beverly Hills, CA: Sage.

Restrepo, M.A. and Silverman, S.W. (2001) Validity of the Spanish Preschool LanguageScale-3 for use with bilingual children. American Journal of SpeechLanguage Pathology, 10, 382-393.

Reynolds, T. (2010). Don't believe the hype: Towards a contextualised understanding of absent black-Caribbean fathers and black boys in family life. Response to Tony Sewell. In Weekes-Bernard, D. (Ed.). Did they get it right? A re-examination of school exclusions and race equality. (p.17-19). London: Runnymede Trust. 
Richardson, R. and Miles, B. (2008). Racist Incidents and Bullying in Schools. Stoke on Trent: Trentham Books.

Robson, C. (2011). Real world research: A resource for social-scientists and practitioner- researchers (3rd Edn). Oxford: Blackwell Publishing.

Rogers, M. R. (1998). The influence of race and consultant verbal behaviour on perceptions of consultant competence and multicultural sensitivity. School Psychology Quarterly, 13, 265-280.

Rogers, M. R. (2000). Examining the cultural context of consultation. School Psychology Review, 29, 414-418.

Rogers, M. R. and Ponterotto, J. G. (1998). Development of the Multicultural School Psychology Counselling Competency Scale. Psychology in Schools, 34, 211-217.

Rollock, N. (2005). Dressed to Fail? Black male students and academic success. Runnymede Quarterly Bulletin, June 2005.

Roseberry-McKibbin, C. (1994). Assessment and Intervention for Children with Limited English Proficiency and Language Disorders. American Journal of Speech Language Pathology, September, 77-88.

Roseberry-McKibbin, C., and Eicholtz, G. E. (1994). Serving limited English proficient children in schools: A national survey. Language, Speech, and Hearing Services in Schools, 25, 156-164.

Roy, W.G. (2001). Making Societies: The Historical Construction of Our World. Thousand Oaks, CA: Pine Forge Press.

Rueda, R., and Mercer, J. (1985). A predictive analysis of decision-making practices with limited English proficient handicapped students. CA: Southwest Regional Laboratory for Educational Research and Development. 
Rutter, J. (2004). Refugee Communities in the UK: Somali Children's Educational Progress and Life Experiences. London Metropolitan University.

Schein, E. H. (1990). Organizational culture. American Psychologist, 43 (2), 109-119.

Schommer-Aikins M. (2002). An evolving theoretical framework for an epistemological belief system. In Hofer, B. and Pintrich. P. (Eds.). Personal epistemology: The psychology of beliefs about knowledge and knowing, (pp. 113-118). Mahwah NJ: Erlbaum.

Schon. D. (1983). The reflective practitioner: Case studies in and on educational practice. New York: Teachers College Press

Schott, T.L.M. and Henley, K.I., (1996). Cultural Aspects of Multiculturalism, Butterworth Heinemann, Oxford,

Scuglik, D. L., and Alarcon, R. D. (2005). Growing up whole: Somali children and adolescents in America. Psychiatry, 2(8), 20-31.

Sewell, T. (15/03/2010). Black boys are too feminised. The Guardian. https://www.theguardian.com/commentisfree/2010/mar/15/black-boys-toofeminised-fathers

Sharma, S. (2005). Multicultural Education: Teachers' Perceptions and Preparation. Journal of College Teaching and Learning, 5(2), 53-63

Shenton, A., K. (2004). Strategies for ensuring trustworthiness in qualitative research projects. Education for Information; 22: 63-75 
Sheridan, S. M. (2000). Considerations of multiculturalism and diversity in behavioral consultation with parents and teachers. School Psychology Review, 29, 389-400.

Silverman, D. (2005). Doing qualitative research (2nd Ed.). London: Sage

Smedley, A. (1993). Race in North America. Boulder, CO: Westview Press.

Smith, D. K. (1984). Practicing school psychologists: Their characteristics, activities and populations served. Professional Psychology, 15, 798-810.

Smith, E. J. (1981). Cultural and historical perspectives in counselling blacks. In D.W. Sue (Ed.), Counselling the culturally different. New York: John Wiley and Sons.

Smith, J.A., Flowers, P., and Larkin, M. (2009). Interpretative phenomenological analysis: theory, method and research. Los Angeles, Sage.

Sodowsky, G. R., Kwan, K. L. and Pannu, R. (1995). Ethnic identity of Asians in the United States. In J. G. Ponterotto et al. (Eds.), Handbook of Multicultural Counseling. Thousand Oaks, CA: Sage.

Sodowsky, G. R., Taffe, R. C., Gutkin, T. B., and Wise, S. L. (1994). Development of the multicultural counseling inventory $(\mathrm{MCl})$ : A self-reported measure of multicultural competencies. Journal of Counseling Psychology, 41, 131-148.

Solorzano, D. and Ornelas, A. (2002). A Critical Race Analysis of Advance Placement Classes: A Case of Educational Inequalities. Journal of Latinos and Education, 1, 215-229. 
Speed, B. (1991). Reality exists O.K.? An argument against constructivism and social constructionism. Journal of Family Therapy, 13(4), 395-409.

Spencer, M. B. and Markstrom-Adams, C. (1990). Identity processes among racial and ethnic minority children in America. Child Development, 61, 290-310.

Spencer-Oatey, H. (2008). Culturally Speaking. Culture, Communication and Politeness Theory. Second Edition. London: Continuum.

Standards for Educational and Psychological Testing (1999). American Psychological Association. Washington, DC: American Educational Research Association.

Stinnett, T. A., Havey, M. J., and Oehler-Stinnett, J. (1994). Current test usage by practicing school psychologists: A national survey. Journal of Psychoeducational Assessment, 12, 331-350.

Stow, C., and Dodd, B., (2003). Providing an equitable service to bilingual children in the UK: a review. International Journal of Language and Communication Disorders, 38, 4, 351-377.

Strand, S. (2009). In-school factors and the White British-Black Caribbean attainment gap: Test, tiers and unintended consequences of assessment practice. Paper presented at the Annual Conference of the American Educational Research Association, San Diego, California, 13-17 April 2009.

Strand, S. (2012). The White British-Black Caribbean achievement gap: Tests, tiers and teacher expectations. British Educational Research Journal, 38(1), 75-101.

Stringer, P., Elliot, J. and Lauchlan, F. (1997). Dynamic Assessment and its potential for educational psychologists Part 2: The zone of next development? Educational Psychologyin Practice, 12 (4), 87-111 
Sue, D. W. (1981). Counselling the culturally different. New York: John Wiley and Sons.

Sue, D. W. (2003). Multidimensional facets of cultural competence. The Counselling Psychologist, 29, 790-821. DOI: $10.1177 / 0011000001296002$

Sue, D. W. (2005). Multicultural Social Work Practice. Hoboken, NJ: John Wiley \& Sons.

Sue, D. W., Capodilupo, C. M., Torino, G. C., Bucceri, J. M., Holder, A. M., Nadal, K. L., and Esquilin, M. E. (2007). Racial microaggressions in everyday life: Implications for counseling. The American Psychologist, 62(4), 271-286. DOI: $\underline{10.1037 / 0003-066 X .62 .4 .271}$

Sue, D.W., Arredondo, P., and McDavis, R.J. (1992). Multicultural counselling competencies and standards: A call to the profession. Journal of Multicultural Counselling and Development, 20, 64-88.

Sue, D.W., Bernier, J.B., Durran, M., Feinberg, L., Pedersen, P., Smith, E., and Vasquez-Nuttall, E. (1982). Position paper: Cross-cultural counseling competencies. The Counseling Psychologist. 10 (2), 45-52.

Sue, S., Zane, N., Hall, G. C. N., and Berger, L.K. (2009). The case for cultural competency in psychotherapeutic interventions. Annual Review of Psychology, 60:525-548.

Sukhnandan, L., and Lee, B. (1998). Streaming, setting and grouping by ability: a review of the literature. Slough, UK: National Foundation for Educational Research in England and Wales.

Swann Report (1985), Education for All, London: HMSO. 
Takaki, R. (1993). A different mirror. Boston, MA: Little, Brown, and Company.

Tashakkori, A. and Teddlie, C. (2003). Handbook of Mixed Methods in Social and Behavioral Research. Thousand Oaks: Sage

Taylor, T.R. and Payne, I. M., (1983). A review of three approaches to cognitive assessment, and a proposed integrated approach based on a unifying theoretical framework. South African Journal of Psychology, 24(4), 184-193.

Thompson, V. L. and Alexander, H. (2006). Therapists' race and African American clients' reactions to therapy. Psychotherapy: Theory, Research,

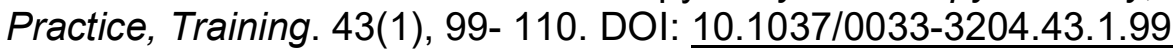

Tuckett, A. G. (2005). Applying thematic analysis theory to practice: A researcher's experience. Contemporary Nurse, 19(1-2), 75-87.

Tzuriel, D. (2001). Not all testing is Dynamic Testing: Overcoming the Fuzzy Identity of Dynamic Assessment. Issues in Education, 7, 211-221.

Ullmann, P. (2009). Inspecting on Race Equality: An Analytical View from the Shires, Race Equality Teaching, 27 (3), 36-41.

Ültanir, E. (2012). An Epistemological Glance at the Constructivist Approach: Constructivist Learning in Dewey, Piaget and Montessori. International Journal of Instruction, 5 (2), 195-212.

Upton, S. J. (2012). Hope and the city: a case study of the resiliency adaptations of British boys of African or Caribbean cultural heritage attending Year 7 at an urban secondary school. Retrieved from ORCA, Cardiff University: https://orca.cf.ac.uk/40797/1/2012UptonJDEdPsy.pdf 
Velez, E. (2002). Multicultural counselling competencies of doctoral psychology interns and their primary supervisors. Unpublished doctoral dissertation, University of Maryland, College Park.

Vera, E. M., and Speight, S. L. (2003). Multicultural competence, social justice, and counseling psychology: Expanding our roles. The Counseling Psychologist, 31, 253-272. DOI: $10.1177 / 0011000003031003001$

von Foerster, H. (1984). On constructing a reality. In P. Watzlawick (Ed.). The invented reality, pp. 41-61). New York: Norton

von Glasersfeld, E. (1988). The reluctance to change a way of thinking. The Irish Journal of Psychology, 9(1), 83-90

Wagner, P. (2008). Consultation as a Framework for Practice. In B. Kelly, L. Woolfson and J. Boyle (Eds.). Frameworks for Practice in Educational Psychology: A Textbook for Trainees and Practitioners. London: Jessica Kingsley

Watt, D. (2007). On Becoming a Qualitative Researcher: The Value of Reflexivity. The Qualitative Report, 12(1), 82-101. Retrieved from: http://www.nova.edu/ssss/QR/QR12-1/watt.pdf

Watts, J. (2008). Emotion, empathy and exit: Reflections on doing ethnographic qualitative research on sensitive topics. Medical Sociology On Line, 3(3), 3-14.

Watzlawick, P. (1984). The Invented reality: How do we know what we believe we know?: Contributions to constructivism (1st ed.). New York: Norton.

Webb, C. (1992). The use of the first person in academic writing: objectivity, language and gatekeeping. Journal of Advanced Nursing, 17(6), 747-52. 
Wenglinsky, H. (2000, October). How teaching matters: Bringing the classroom back into discussions of teacher quality. Princeton, NJ: The Milken Family Foundation and Educational Testing Service.

Whaley, A. L. (2008). Cultural sensitivity and cultural competence: toward clarity of definitions in cross-cultural counselling and psychotherapy. Counselling Psychology Quarterly, 21(3), 215-222. DOI: $\underline{10.1080 / 09515070802334781}$

Winter, K. (1999). Speech and language therapy provision for bilingual children: aspects of the current service. International Journal of Language and Communication Disorders. 34, 85-98.

Wohland P., Rees P., Norman P., Boden P., and Jasinska M. (2010). Ethnic population projections for the UK and local areas, 2001-2051. Working Paper 10/02, School of Geography, University of Leeds, June 2010. Online at: http://www.geog.leeds.ac.uk/fileadmin/documents/research/csap/10-02.pdf

Wray, M. and Newitz, A. (1997). White trash: Race and class in America. New York: Routledge.

WYATT, T., 1998, Assessment Issues with Multicultural Populations. In D. Battle (ed.), CommunicationDisorders in Multicultural Populations (Boston: Butterworth-Heinemann), pp. 379-425

Yeh, M., Eastman, K. and Cheung, M. K. (1994). Children and adolescents in community health centers: Does the ethnicity or the language of the therapist matter? Journal of Community Psychology, 22,153-163.

Ysseldyke, J., Burns, M., Dawson, P. Kelly, B., Morrison, D., Ortiz, S., Rosenfield, S., \& Telzrow, C. (2006). School Psychology: A Blueprint for Training and Practice III. Bethesda, MD: National Association of School Psychologist

Zhou, Z., Bray, M., Kehle, T., Theodore, L., Clark, E. and Jenson, W. (2004). Achieving ethnic minority parity in School Psychology. Psychology in the Schools, 41, 443-450. 
Zirkel, S. (2008). Creating More Effective Multi-ethnic Schools. Social Issues and Policy Review, 2: 187-241. DOI: 10.1111/j.1751-2409.2008.00015.x 
Appendices 


\section{Appendix I}

Summary of Articles Generated and Selected from Electronic Databases, using a Range of Search Terms.

\begin{tabular}{|c|c|c|c|}
\hline$\overbrace{0}^{\pi}$ & Search Terms & Results (N) & Articles Selected $(\mathrm{N})$ \\
\hline 覂 & "School Psychologist" and "Multicultural" & 44 & 2 \\
\hline 궁 & "Educational Psychologist" and "Multicultural" & 17 & 1 \\
\hline 崖 & "School Psychologist" and "Multicultural" and "Competence" & 9 & 3 \\
\hline$\underline{\underline{u}}$ & "Educational Psychologist" and "Multicultural" and "Competence" & 5 & 0 \\
\hline$\frac{5}{0}$ & "School Psychologist" and "Multicultural" and "Assessment" & 9 & 0 \\
\hline 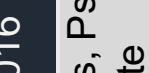 & "Educational Psychologist" and "Multicultural" and "Assessment" & 0 & n/a \\
\hline$\frac{\infty}{0} \frac{\pi}{0}$ & "School Psychologist" and "Assessment" and "Minorities" & 2 & 1 \\
\hline 焉 $\bar{g}$ & "Educational Psychologist" and "Assessment" and "Minorities" & 2 & 1 \\
\hline 每 & "School Psychologist" and "Assessment" and "Black" & 4 & 0 \\
\hline $0 . \frac{2}{\pi}$ & "Educational Psychologist" and "Assessment" and "Black" & 0 & $\mathrm{n} / \mathrm{a}$ \\
\hline$\frac{0}{x} \underset{\sim}{\infty}$ & "School Psychologist" and "Consultation" and "Diversity" & 11 & 0 \\
\hline Ш & "Educational Psychologist" and "Consultation" and "Diversity" & 6 & 1 \\
\hline$\frac{\Phi}{0}$ & "School Psychologist" and "Assessment" and "Multiculturalism" & 2 & 0 \\
\hline 产 & "Educational Psychologist" and "Assessment" and "Multiculturalism" & 0 & $\mathrm{n} / \mathrm{a}$ \\
\hline $\begin{array}{c}\infty \\
\infty \\
\infty\end{array}$ & "School Psychologist" and "Consultation" and "Multiculturalism" & 5 & 1 \\
\hline 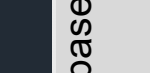 & "Educational Psychologist" and "Consultation" and "Multiculturalism" & 0 & n/a \\
\hline$\frac{\frac{\pi}{\pi}}{\pi}$ & "School Psychologist" and "Assessment" and "Bilingual" & 16 & 6 \\
\hline ص & "Educational Psychologist" and "Assessment" and "Bilingual" & 6 & 1 \\
\hline
\end{tabular}


Overview of Articles Selected for Literature Review

\section{Research Studies}

\begin{tabular}{|c|c|c|c|c|c|c|c|}
\hline $\begin{array}{l}\text { Author(s) } \\
\text { and Date }\end{array}$ & Title & $\begin{array}{c}\text { Research } \\
\text { Location }\end{array}$ & Participants & Methodology & Aims of Research & Findings & Strengths/Limitations \\
\hline $\begin{array}{l}\text { Figueroa } \\
\text { and } \\
\text { Newsom } \\
\text { e } \\
(2006)\end{array}$ & $\begin{array}{l}\text { The Diagnosis } \\
\text { of LD in } \\
\text { English } \\
\text { Language } \\
\text { Learners: is it } \\
\text { Non- } \\
\text { discriminatory } \\
?\end{array}$ & USA & $\begin{array}{l}19 \text { diagnostic } \\
\text { folders chosen } \\
\text { on the basis of } \\
\text { SLD diagnosis, } \\
\text { English language } \\
\text { proficiency } \\
\text { scores below } \\
\text { fluent English } \\
\text { speaking and a } \\
\text { home language } \\
\text { designation of } \\
\text { "other than } \\
\text { English" }\end{array}$ & Quantitative & $\begin{array}{l}\text { Examining the non- } \\
\text { discriminatory } \\
\text { diagnosis of } \\
\text { learning } \\
\text { disabilities in } \\
\text { English learners. }\end{array}$ & $\begin{array}{l}\text { Writers of psychological reports- } \\
\text { school psychologists- do not } \\
\text { assess or investigate the possible } \\
\text { confounding effects of bilingualism } \\
\text { on tests, testing and diagnoses. }\end{array}$ & $\begin{array}{l}19 \text { reports written by just } 6 \text { school } \\
\text { psychologists across } 5 \text { districts in } \\
\text { California and low sample size; } \\
\text { generalisability is a concern. } \\
\text { Findings mirror others (Klingner and } \\
\text { Harry, 2006; Wilkinson, Oritz, } \\
\text { Robertson and Kushner, 2006). }\end{array}$ \\
\hline $\begin{array}{c}\text { McClosk } \\
\text { eyAthana } \\
\text { siou } \\
(2000)\end{array}$ & $\begin{array}{l}\text { Assessment } \\
\text { and } \\
\text { intervention } \\
\text { practices with } \\
\text { second- } \\
\text { language } \\
\text { learners } \\
\text { among school } \\
\text { psychologists. }\end{array}$ & USA & $\begin{array}{l}\text { A } 10 \text {-item survey } \\
\text { with a } 33 \% \text { of } \\
\text { surveys returned } \\
\text { and considered } \\
\text { for analysis. }\end{array}$ & Quantitative & $\begin{array}{l}\text { To gain } \\
\text { information about } \\
\text { assessment } \\
\text { instruments and } \\
\text { procedures and } \\
\text { intervention } \\
\text { practices used by } \\
\text { school } \\
\text { psychologists with } \\
\text { linguistically } \\
\text { different children. }\end{array}$ & $\begin{array}{l}\text { Although some SPs use alternative } \\
\text { assessment techniques, the } \\
\text { majority continue to use traditional } \\
\text { cognitive tests (Weschler Scales) } \\
\text { and traditional interventions } \\
\text { (resource room service delivery). }\end{array}$ & $\begin{array}{l}\text { Results based on just } 33 \% \text { of the state } \\
\text { school psychology association } \\
\text { membership- results not truly } \\
\text { representative for all school } \\
\text { psychologists in state or nationally. } \\
\text { Respondents may be more interested } \\
\text { in topic than those not interested. } \\
\text { Not all respondents responded to all } \\
\text { questions- more/less respondents for } \\
\text { different questions may skew } \\
\text { responses. } \\
\text { Issues around terminology used- } \\
\text { unclear for some respondents, e.g. } \\
\text { SLL. }\end{array}$ \\
\hline
\end{tabular}




\begin{tabular}{|c|c|c|c|c|c|c|c|}
\hline $\begin{array}{l}\text { Author(s) } \\
\text { and Date }\end{array}$ & Title & $\begin{array}{l}\text { Research } \\
\text { Location }\end{array}$ & Participants & Methodology & Aims of Research & Findings & Strengths/Limitations \\
\hline $\begin{array}{l}\text { Bainter } \\
\text { and } \\
\text { Tollefson } \\
(2003)\end{array}$ & $\begin{array}{l}\text { Intellectual } \\
\text { assessment of } \\
\text { language } \\
\text { minority } \\
\text { students: What } \\
\text { do school } \\
\text { psychologists } \\
\text { believe are } \\
\text { acceptable } \\
\text { practices? }\end{array}$ & USA & $\begin{array}{l}\text { Survey sent to } \\
500 \text { school } \\
\text { psychologists in } \\
8 \text { states- } 195 \\
\text { respondents } \\
\text { participated. }\end{array}$ & Quantitative & $\begin{array}{l}\text { To examine the } \\
\text { acceptability of } \\
\text { methods used to } \\
\text { assess the } \\
\text { cognitive ability of } \\
\text { language minority } \\
\text { students. }\end{array}$ & $\begin{array}{l}\text { Using a bilingual school } \\
\text { psychologist or tests when English } \\
\text { was the dominant language in a } \\
\text { SLL were usually or always } \\
\text { acceptable. Non-verbal tests or } \\
\text { foreign-normed tests were } \\
\text { considered sometimes or usually } \\
\text { acceptable. However, } \\
\text { administering tests in English when } \\
\text { a student was dominant in another } \\
\text { language and using non-verbal } \\
\text { tests that required oral instructions, } \\
\text { without the use of an interpreter, } \\
\text { were considered never or rarely } \\
\text { acceptable. }\end{array}$ & $\begin{array}{l}\text { Sample skewed towards those } \\
\text { interested/concerned with the area of } \\
\text { study vs those not. }\end{array}$ \\
\hline $\begin{array}{l}\text { O'Bryon } \\
\text { and } \\
\text { Rogers } \\
(2010)\end{array}$ & $\begin{array}{l}\text { Bilingual } \\
\text { school } \\
\text { psychologists' } \\
\text { assessment } \\
\text { practices with } \\
\text { English } \\
\text { language } \\
\text { learners }\end{array}$ & USA & $\begin{array}{l}1000 \text { bilingual } \\
\text { NASP members } \\
\text { were recruited } \\
\text { nationwide- } 276 \\
\text { participated. }\end{array}$ & Quantitative & $\begin{array}{l}\text { An exploration of } \\
\text { bilingual school } \\
\text { psychologists' } \\
\text { assessment } \\
\text { practices with } \\
\text { students identified } \\
\text { as English } \\
\text { language learners. }\end{array}$ & $\begin{array}{l}\text { Of those who conducted language } \\
\text { proficiency assessments of ELLs, } \\
58 \% \text { used comprehensive methods } \\
\text { of assessments. Those who did } \\
\text { use interpreters reported a mix of } \\
\text { best practices and discouraged } \\
\text { practices for their use. Participants } \\
\text { reported that engaging in } \\
\text { continuing education activities } \\
\text { about assessing ELLs predicted } \\
\text { best practices as well as engaging } \\
\text { in applied preservice training with } \\
\text { bilingual supervision and best } \\
\text { practice behaviours assessing } \\
\text { student acculturation. }\end{array}$ & $\begin{array}{l}\text { Although deemed truly proficient as a } \\
\text { bilingual practitioner based on their } \\
\text { NASP membership, } 1 / 3 \text { of } \\
\text { participants did not respond to } \\
\text { questions about their fluency in either } \\
\text { language. } \\
\text { Those not self-identifying as fluent } \\
\text { were rejected from further analyses- } \\
\text { significantly effecting sample size and } \\
\text { generalisability. } \\
10 \% \text { reported using ASL as an } \\
\text { additional language- not relevant for } \\
\text { the current study. } \\
\text { Format of survey questions was a } \\
\text { limitation: other formats could have } \\
\text { been used. }\end{array}$ \\
\hline
\end{tabular}




\begin{tabular}{|c|c|c|c|c|c|c|c|}
\hline $\begin{array}{l}\text { Author(s) } \\
\text { and Date }\end{array}$ & Title & $\begin{array}{l}\text { Research } \\
\text { Location }\end{array}$ & Participants & Methodology & Aims of Research & Findings & Strengths/Limitations \\
\hline $\begin{array}{c}\text { Ochoa, } \\
\text { Rivera } \\
\text { and } \\
\text { Ford } \\
\text { (1997) }\end{array}$ & $\begin{array}{l}\text { An } \\
\text { investigation } \\
\text { of school } \\
\text { psychology } \\
\text { training } \\
\text { pertaining to } \\
\text { bilingual } \\
\text { psycho- } \\
\text { educational } \\
\text { assessment of } \\
\text { primarily } \\
\text { Hispanic } \\
\text { students: } \\
\text { Twenty-five } \\
\text { years after } \\
\text { Diana v. } \\
\text { California }\end{array}$ & USA & $\begin{array}{l}\text { Survey of } 1,507 \\
\text { school } \\
\text { psychologists } \\
\text { from eight states. }\end{array}$ & Quantitative & $\begin{array}{l}\text { Examine the extent } \\
\text { to which school } \\
\text { psychology } \\
\text { programmes } \\
\text { provide training in } \\
\text { bilingual psycho- } \\
\text { educational } \\
\text { assessment. }\end{array}$ & $\begin{array}{l}\text { School psychologists who conduct } \\
\text { bilingual psycho-educational } \\
\text { assessment do not believe that they } \\
\text { have received adequate training. }\end{array}$ & $\begin{array}{l}\text { Majority of sample was white ( } 87 \%)- \\
\text { only } 13 \% \text { comprised minorities- not } \\
\text { entirely representative. } \\
\text { Sample skewed towards those } \\
\text { interested/concerned with the area of } \\
\text { study vs those not. }\end{array}$ \\
\hline $\begin{array}{l}\text { Jitendra } \\
\text { and } \\
\text { Rohena- } \\
\text { Diaz } \\
(1996)\end{array}$ & $\begin{array}{l}\text { Language } \\
\text { Assessment of } \\
\text { Students who } \\
\text { are } \\
\text { Linguistically } \\
\text { Diverse: Why } \\
\text { a Discrete } \\
\text { Approach is } \\
\text { Not the } \\
\text { Answer }\end{array}$ & USA & $\begin{array}{l}\text { Case study of an } \\
\text { 8-year-old } \\
\text { Puerto Rican boy } \\
\text { whose teacher } \\
\text { believed he } \\
\text { needed referral } \\
\text { to special } \\
\text { education is } \\
\text { presented to } \\
\text { demonstrate the } \\
\text { steps involved in } \\
\text { the assessment } \\
\text { of language } \\
\text { proficiency }\end{array}$ & Qualitative & $\begin{array}{l}\text { Review of relevant } \\
\text { issues from the } \\
\text { fields of bilingual } \\
\text { and special } \\
\text { education as they } \\
\text { relate to the } \\
\text { language } \\
\text { assessment of } \\
\text { students who are } \\
\text { linguistically } \\
\text { diverse. }\end{array}$ & $\begin{array}{l}\text { Recommendations for the valid use } \\
\text { of language assessment to better } \\
\text { inform school psychologists who } \\
\text { interact with speech language } \\
\text { pathologists involved in the } \\
\text { language assessment of } \\
\text { linguistically diverse students. }\end{array}$ & $\begin{array}{l}\text { Single case study- issues of } \\
\text { generalisability. }\end{array}$ \\
\hline
\end{tabular}




\begin{tabular}{|c|c|c|c|c|c|c|c|}
\hline $\begin{array}{l}\text { Author(s) } \\
\text { and Date }\end{array}$ & Title & $\begin{array}{c}\text { Research } \\
\text { Location }\end{array}$ & Participants & Methodology & Aims of Research & Findings & Strengths/Limitations \\
\hline $\begin{array}{l}\text { Rogers } \\
\text { and } \\
\text { Close } \\
(1992)\end{array}$ & $\begin{array}{l}\text { Multicultural } \\
\text { training in } \\
\text { school } \\
\text { psychology: a } \\
\text { national } \\
\text { survey. }\end{array}$ & USA & $\begin{array}{l}\text { Survey data of } \\
\text { Directors of } 121 \\
\text { school } \\
\text { psychology } \\
\text { training program } \\
\text { in the US. }\end{array}$ & Quantitative & $\begin{array}{l}\text { To measure the } \\
\text { extent to which } \\
\text { programs } \\
\text { integrated } \\
\text { multicultural } \\
\text { themes into core } \\
\text { courses, offered } \\
\text { minority-related } \\
\text { courses, exposed } \\
\text { students to } \\
\text { culturally diverse } \\
\text { clients during } \\
\text { practical and } \\
\text { internships, } \\
\text { provided minority- } \\
\text { issues research } \\
\text { opportunities and } \\
\text { represented } \\
\text { culturally diverse } \\
\text { groups among } \\
\text { faculty and } \\
\text { students. }\end{array}$ & $\begin{array}{l}\text { APA-accredited and PhD-granting } \\
\text { programs were making the most } \\
\text { effort to include minority themes in } \\
\text { assessment coursework, offer } \\
\text { optional minority related courses, } \\
\text { and involve students in minority } \\
\text { issues research projects. However, } \\
40 \% \text { of the programs sampled did } \\
\text { not offer specific courses in } \\
\text { minority issues or integrate } \\
\text { multicultural content/themes into } \\
\text { core school psychology courses. } \\
\text { Programs in more heavily } \\
\text { populated areas provided more } \\
\text { fieldwork with a culturally diverse } \\
\text { clientele and more diverse chances } \\
\text { to work beside a racial/ethnic } \\
\text { minority classmates. }\end{array}$ & $\begin{array}{l}\text { Survey data skewed towards those } \\
\text { interested/concerned with the area of } \\
\text { study vs those not. } \\
\text { Focus on US population- findings } \\
\text { likely to vary among training course } \\
\text { directors in the UK. }\end{array}$ \\
\hline $\begin{array}{l}\text { Kearns, } \\
\text { Ford } \\
\text { and } \\
\text { Linney } \\
(2005)\end{array}$ & $\begin{array}{l}\text { African } \\
\text { American } \\
\text { student } \\
\text { representation } \\
\text { in special } \\
\text { education } \\
\text { programs. }\end{array}$ & USA & $\begin{array}{l}600 \text { school } \\
\text { psychologists } \\
\text { randomly } \\
\text { selected and } \\
\text { targeted for } \\
\text { participation in } \\
\text { current study. } \\
129 \text { valid survey } \\
\text { responses } \\
\text { obtained. A } \\
\text { further } 22 \text { school } \\
\text { psychologists } \\
\text { recruited after } \\
\text { follow-up } \\
\text { correspondence. } \\
\text { Total } 151 \text { school } \\
\text { psychologist } \\
\text { participants. }\end{array}$ & $\begin{array}{c}\text { Mixed methods- } \\
\text { quantitative } \\
\text { and } \\
\text { qualitative } \\
\\
\end{array}$ & $\begin{array}{l}\text { To obtain } \\
\text { information from } \\
\text { the school } \\
\text { psychologists } \\
\text { perspectives on } \\
\text { the } \\
\text { disproportionate } \\
\text { representation of } \\
\text { African American } \\
\text { students in special } \\
\text { education } \\
\text { programmes for } \\
\text { students with mild } \\
\text { disabilities. }\end{array}$ & $\begin{array}{l}\text { Participants considered lack of } \\
\text { parental involvement and broadly } \\
\text { defined cultural disadvantage, the } \\
\text { failures of both the regular } \\
\text { education and special education } \\
\text { systems and pressures from } \\
\text { parents and teachers to place } \\
\text { African American students as the } \\
\text { most influential factors that } \\
\text { represent African American } \\
\text { students in special education. } \\
\text { Moreover, the self-reported cross- } \\
\text { cultural competence of participants } \\
\text { was found to be associated with the } \\
\text { likelihood that solutions to the } \\
\text { above named problems would be } \\
\text { implemented. }\end{array}$ & $\begin{array}{l}\text { Sample skewed towards those } \\
\text { interested/concerned with the area of } \\
\text { study vs those not. } \\
\text { Respondents were mostly Caucasian } \\
(87.9 \%)\end{array}$ \\
\hline
\end{tabular}




\section{Journal Articles}

\begin{tabular}{|c|c|c|c|c|}
\hline $\begin{array}{c}\text { Author(s) and } \\
\text { Date }\end{array}$ & Title & Setting & Source & Article Summary \\
\hline $\begin{array}{l}\text { Sheridan, S.M. } \\
\qquad(2000)\end{array}$ & $\begin{array}{l}\text { Considerations of } \\
\text { Multiculturalism and } \\
\text { Diversity in Behavioral } \\
\text { Consultation with } \\
\text { Parents and Teachers }\end{array}$ & USA & $\begin{array}{l}\text { School } \\
\text { Psychology } \\
\text { Review, 29(3), } \\
344-353\end{array}$ & $\begin{array}{l}\text { To explore the structural and relational issues that may develop when working with } \\
\text { culturally diverse students and their families when considering essential elements of } \\
\text { conjoint/behavioural consultation. } \\
\text { Individual differences within and between cultural groups are at the heart of } \\
\text { multicultural models and must be considered within relevant systemic contexts. } \\
\text { Awareness/sensitivity of the consultant, the ability to consider various frames of } \\
\text { reference and procedures to implement these effectively into empirically validated } \\
\text { C/BC models are essential. }\end{array}$ \\
\hline $\begin{array}{l}\text { Ingraham, C.L. } \\
\qquad(2000)\end{array}$ & $\begin{array}{l}\text { Consultation Through a } \\
\text { Multicultural Lens: } \\
\text { Multicultural and Cross- } \\
\text { Cultural Consultation in } \\
\text { Schools }\end{array}$ & USA & $\begin{array}{l}\text { School } \\
\text { Psychology } \\
\text { Review, 29(3), } \\
320-343\end{array}$ & $\begin{array}{l}\text { To (1) use a multicultural lens to explore the practice of multicultural and cross-cultural } \\
\text { consultation in schools; and (2) identify issues, constructs, and processes to explore in } \\
\text { future research. } \\
\text { Multicultural school consultation (MSC) offers a conceptual framework to explore } \\
\text { structures, processes, and interrelationships among consultation members. The } 5 \\
\text { components of MSC include domains for consultant learning and development, } \\
\text { consultee learning and development, cultural diversity in consultation constellations, } \\
\text { contextual and power influences, and methods to support consultee and client } \\
\text { success. }\end{array}$ \\
\hline $\begin{array}{l}\text { Rogers, M.R. } \\
\qquad(2000)\end{array}$ & $\begin{array}{l}\text { Examining the Cultural } \\
\text { Context of Consultation }\end{array}$ & USA & $\begin{array}{l}\text { School } \\
\text { Psychology } \\
\text { Review, 29(3), } \\
441-418\end{array}$ & $\begin{array}{l}\text { Presenting a conceptual model of multicultural consultation, advance the study of } \\
\text { culture in school psychology inquiry, and contribute to the understanding of cross- } \\
\text { cultural consultation competencies important to the successful delivery of consultation } \\
\text { services. } \\
\text { Cross-Cultural Consultation Competencies identified: Understanding one's own and } \\
\text { others culture, developing CC communication and interpersonal skills, examining the } \\
\text { cultural embeddedness of consultation, using qualitative methodologies, acquiring } \\
\text { culture-specific knowledge and understanding and skill in working with interpreters. }\end{array}$ \\
\hline
\end{tabular}




\begin{tabular}{|c|c|c|c|c|}
\hline $\begin{array}{l}\text { Author(s) and } \\
\text { Date }\end{array}$ & Title & Setting & Source & Article Summary \\
\hline $\begin{array}{l}\text { American } \\
\text { Psychological } \\
\text { Association } \\
(2003)\end{array}$ & $\begin{array}{l}\text { Guidelines on } \\
\text { multicultural education, } \\
\text { training, research, } \\
\text { practice and } \\
\text { organisational change } \\
\text { for Psychologists }\end{array}$ & USA & $\begin{array}{l}\text { American } \\
\text { Psychologist, } \\
\text { 58(5), 377-402 }\end{array}$ & $\begin{array}{l}\text { Guidelines to provide psychologists with (a) the rationale and needs for addressing } \\
\text { multiculturalism and diversity in education, training, research, practice, and } \\
\text { organizational change; (b) basic information, relevant terminology, current empirical } \\
\text { research from psychology and related disciplines, and other data that support the } \\
\text { proposed guidelines and underscore their importance; (c) references to enhance } \\
\text { on-going education, training, research, practice, and organizational change } \\
\text { methodologies; and (d) paradigms that broaden the purview of psychology as a } \\
\text { profession. }\end{array}$ \\
\hline $\begin{array}{l}\text { Ehrhardt- } \\
\text { Padgett, G.N., } \\
\text { Hatzichristou, } \\
\text { C., } \\
\text { Kitson, J. } \\
\text { and Meyers, J. } \\
\text { (2004) }\end{array}$ & $\begin{array}{l}\text { Awakening to a New } \\
\text { Dawn: Perspectives of } \\
\text { the Future of School } \\
\text { Psychology }\end{array}$ & USA & $\begin{array}{l}\text { School } \\
\text { Psychology } \\
\text { Review, 33(1), } \\
\text { 105-114 }\end{array}$ & $\begin{array}{l}\text { To share combined perspectives of the future of school psychology, including } \\
\text { suggestions for the future direction of school psychology, and encouragement for all } \\
\text { school psychologists to awaken to a change in the field. The article considers the } \\
\text { educational and mental health needs of students, and offers a prospectus for the field } \\
\text { including (a) data-supported services (b) group-focused practices (c) collaboration } \\
\text { and partnerships with professionals, families, and schools and (d) multicultural } \\
\text { considerations. }\end{array}$ \\
\hline $\begin{array}{l}\text { Markeda, M. } \\
\text { L., } \\
\text { Nastasi, K B., } \\
\text { Hatzichristou, } \\
\text { C., } \\
\text { Jones, J.M., } \\
\text { Schanding, Jr., } \\
\text { T.G., } \\
\text { and Yetter, G. } \\
\text { (2010) }\end{array}$ & $\begin{array}{l}\text { Evidence on } \\
\text { multicultural training in } \\
\text { school psychology: } \\
\text { recommendations for } \\
\text { future research. }\end{array}$ & USA & $\begin{array}{l}\text { School } \\
\text { Psychology } \\
\text { Quarterly, 25(4), } \\
249-278\end{array}$ & $\begin{array}{l}\text { To advance multicultural training in school psychology, guidance is needed regarding } \\
\text { the content and structure necessary to adequately develop multicultural competence. } \\
\text { This article proposes to critically review the evidence on multicultural training in school } \\
\text { psychology and provide a synthesis of the best evidence for preparing multi-culturally } \\
\text { competent school psychologists. }\end{array}$ \\
\hline $\begin{array}{l}\text { Lopez, E.C } \\
\text { and } \\
\text { Bursztyn, A.M. } \\
(2013)\end{array}$ & $\begin{array}{l}\text { Future challenges and } \\
\text { opportunities: Towards } \\
\text { culturally responsive } \\
\text { training in school } \\
\text { psychology }\end{array}$ & USA & $\begin{array}{l}\text { Psychology in } \\
\text { Schools, } 50(3), \\
212-228\end{array}$ & $\begin{array}{l}\text { Training and field experiences that are grounded in multicultural practices, research, } \\
\text { and advocacy will foster greater competence in addressing diverse schools', } \\
\text { students', and families' needs. However, there are four major program challenges to } \\
\text { advancing culturally responsive preparation of school psychologists. These include (a) } \\
\text { integrating multicultural perspectives within the philosophical foundation of training } \\
\text { programs, (b) adapting multicultural approaches to reframe education and } \\
\text { psychological theory in school psychology training, (c) defining the multicultural scope } \\
\text { for training, and (d) articulating and implementing multicultural competencies via } \\
\text { criteria already sanctioned by our profession through the latest National Association of } \\
\text { School Psychologists Standards for Graduate Preparation of School Psychologists. }\end{array}$ \\
\hline
\end{tabular}




\begin{tabular}{|c|l|c|l|l|}
\hline $\begin{array}{c}\text { Author(s) and } \\
\text { Date }\end{array}$ & \multicolumn{1}{|c|}{ Title } & Setting & \multicolumn{1}{|c|}{ Source } & Article Summary \\
\hline $\begin{array}{c}\text { Gopaul- } \\
\begin{array}{c}\text { McNicol, S-A. } \\
(2001)\end{array}\end{array}$ & $\begin{array}{l}\text { Training Urban School } \\
\text { Counselors and } \\
\text { psychologists to Work } \\
\text { with Culturally, } \\
\text { Linguistically, Urban, } \\
\text { and Ethnically Diverse } \\
\text { Populations }\end{array}$ & USA & $\begin{array}{l}\text { Multicultural } \\
\text { Review, 10(30), } \\
38-73\end{array}$ & $\begin{array}{l}\text { Many psychology graduates secure employment in urban and suburban school } \\
\text { districts in the United States. These areas are quite diverse with respect to culture and } \\
\text { language. In the largest cities in North America, as many as 150 languages are } \\
\text { spoken. }\end{array}$ \\
\hline $\begin{array}{c}\text { Malone, C.M. } \\
(2010)\end{array}$ & $\begin{array}{l}\text { Evaluating multicultural } \\
\text { competence in school } \\
\text { psychology }\end{array}$ & USA & $\begin{array}{l}\text { Communiqué, } \\
38(7), 15-16\end{array}$ & $\begin{array}{l}\text { limportant to train students to have knowledge of individual differences and to be able } \\
\text { to apply this knowledge to the science and practice of psychology. A description and } \\
\text { discussion of the Multicultural School Psychology Counselling Competency Scale } \\
\text { (MSPCCS) and how this can address the settings in which school psychologists } \\
\text { function and the population with which they work. }\end{array}$ \\
\hline
\end{tabular}




\section{Appendix II}

\section{Specific Challenges in Assessment - Language Skills}

There is no doubt that language and cognitive ability are inextricably linked (Schott and Henley 1996, Battle 1998). Those that are bilingual have several cognitive advantages over their non-bilingual peers, ranging from above-average intelligence (Peal and Lambert, 1962) in terms of enhanced executive control which facilitates cognitive flexibility and processing mechanisms (Nayak, Hanson, Kreugar and McLaughlin, 1990) and, selective attention (Bialystok, 2001).

Yet, despite these advantages, there is widespread concern about the most effective ways to educate bilingual children as they often enter school with language skills that are unlike those of their monolingual peers (Goldstein, 2004; Oller and Eilers, 2002). Children who begin learning a second language at the time of first contact with the educational system are at particular risk for misdiagnosis with language impairment (LI) and other learning and cognitive difficulties (Cline and Frederickson, 1996). For example, the functional demand for learning English for many bilingual children growing up in the United States (the majority of whom are Spanish-English bilinguals), begins when they enter the educational system. This group of children typically begin learning English as an additional language (EAL) between three and six years of age as preschoolers. Here, the amount of time of exposure and specific content of the first and second languages influence acquisition patterns and, as such, the home and school contexts shape the focus of conversations and interactions in different ways (Bedore and Peña, 2008). Thus, children in the early stages of bilingual development are likely to have language skills that are in flux (Genesee, 2004). As the second language gradually becomes more complex, the first language may stall or become less complex (Anderson, 1999). At the same time, the level of development in the second language is not typically comparable to that of monolingual speakers of that language. These children are likely to score in the at-risk range on a language and ability assessments in their weaker language. Some children may also score in the at-risk range in their stronger language

during this transition to a second language (Mennen, Stansfield and Johnston, 2005). 
For many teachers and educational policy makers, the supremacy of English both as the medium of education and as a world language can often make it difficult to appreciate or understand the significance of bilingualism (Gravelle, 1996). As such, when children from linguistically diverse backgrounds are seen to be struggling with aspects of the curriculum, a common assumption may be that there is a problem with communication (Cline and Frederickson, 1996) even though bilingual children are just as likely to have special educational needs (SEN) or learning difficulties (e.g. dyslexia and developmental aphasia) as monolingual children. As a result of this, when bilingual children were thought to have special needs or learning difficulties at school, they were often referred to an EP, who carried out a normative intelligence test and concluded that the child's disabilities were due to a lack of proficiency in the second language (Figueroa, 2002).

Advocates of standardised assessment (SA) believe that using such tests serve the function of separating the normal population from the disordered population; testing a child to discover their strengths and weaknesses relative to its peers. They would also argue that a numerical score permits statistical analysis which rules out elements of chance in the result and places the child's performance in relation to the performance of its peers (Baker, 2008). However, the argument against this is that an emergent bilingual child at the nonverbal or telegraphic and formulaic stage of acquiring EAL, whose performance is compared to its monolingual English peers of the same chronological age, can only fail and achieve low scores. Here, the child may fail for several years before reaching a similar level as its peers (Duncan, 1989). Subsequently, any language or ability assessments conducted during this stage of development will result in misleading information that underestimates the child's true capacity. Conversely, the child may never reach the same level as its peers depending on how the monolingual assessment and norms were established (Duncan, 1989). Gravelle (1996) states that SAs in any language remain biased in favour of individuals for whom that language is native and, as such, low test scores received by bilinguals are often interpreted as evidence of deficits or even disorders. As a result of this, the language gap in testing has often been a major contributor to the disproportionate numbers of Hispanic bilinguals diagnosed as mentally retarded in need of remedial education when cut-off scores on IQ tests are used (Duran, 1988). In a 
study of Hispanic children in California, Rueda and Mercer (1985) found that the Hispanic students, who constituted fewer than $10 \%$ of the school population, comprised $32 \%$ of the students identified as mentally retarded. In fact, other data from the study suggest that for over $62 \%$ of the Hispanic students identified as mentally retarded, no symptoms of deficiency were found other than the low IQ test scores (Rueda, 1987 in Duran, 1988).

With traditional cognitive tests, i.e. the Weschler Intelligence Scale for ChildrenThird Edition (WISC III) appearing to hold dominance in assessment materials among the majority of school psychologists in their assessments of linguistically diverse students (Mccloskey and Athanasiou, 2000), to counteract the negative impact of these on the bilingual school population, the use of translation in test adaptation and the development of local norms have commonly been suggested for the assessment of bilingual children (Stow and Dodd, 2003). While these steps have served as the impetus to develop materials in the languages of the local population, there are two main problems with test translation with respect to assessment of learning difficulties. First, translation of language tests assumes that language development in other languages follows the same developmental trajectory. Second, translated tests target linguistic forms that are markers of learning difficulties in the source language but omit important morphosyntactic forms, features of vocabulary use and narrative components in the target language that potentially differentiate performance of children with and without learning difficulties (Taylor and Payne, 1983). Lack of normative data on typical bilingual acquisition or early sequential bilingual development further exacerbates current standardised test limitations.

Language development milestones cannot be assumed to follow the same developmental trajectories in the same order across languages. While there are documented similarities in language acquisition across languages at a general level, there are differences in the specific forms that children learn depending on the language(s) being acquired. In language test development an example can be found in the first adaptation of the Preschool LanguageScale-3 (PLS-3) from English to Spanish, which was essentially a translation. An analysis of the translated items from the English and Spanish versions of the PLS-3 demonstrated that such items had different difficulty levels (Rest repo and 
Silverman, 2001). For example, prepositions were easier in English than in Spanish, but object functions were more difficult in English than in Spanish. The question of appropriate targets for identification of learning difficulties is particularly important when developing assessment tools for bilingual populations. Educational Psychologists must use tests to determine whether impairment exists (Restrepo and Silverman, 2001) and thus, when tests are adapted from English to other languages, it is important to note that the adaptations often do not consider markers of learning difficulties for the target language. Thus, adaptations are very likely to have poorer classification accuracy than the original English tests.

Despite these findings, in examining the acceptability of methods used to assess the cognitive ability of language minority students, Bainter and Tollefson (2003) found that school psychologists believed that using a bilingual school psychologist or tests when English was the dominant language in a second language learner was usually or always acceptable and non-verbal tests or foreign-normed tests were considered sometimes or usually acceptable. However, school psychologists felt that administering tests in English when a student was dominant in another language and using non-verbal tests that required oral instructions, without the use of an interpreter, were considered never or rarely acceptable. Yet, the use of an interpreter when working with students who are English language learners also presents a major challenge for school psychologists. Here, the interpreter may enhance or remove information that the assessor did not wish to express (Lopez, 2002), struggle to interpret concepts that lack equivalence in the examinee's language (Sattler, 2001) and the difficulty level of words may not translate to the same difficulty level in another language (Lopez, 2002). In addition, to uphold impartiality, the interpreter must not have a former personal relationship with the examinee which is difficult if there are few people speaking the same language in a community (American Educational Research Association [AERA], 1999). Furthermore, interpreters may not be adequately aware of standardisation procedures for instruments and the assessment process in general (AERA, 1999). Despite this, the majority of school psychologist (78\%) report using interpreters, with only $52 \%$ having had training in using interpreters appropriately (Ochoa, Riccio, Jimenez, Garcia de Alba and Sinez, 2004). This may be why school psychologists discouraged the 
use of interpreters with English language Learners (ELLs) and report that engaging in continuing education activities about assessing ELLs predicts best practices as well as engaging in applied preservice training with bilingual supervision and best practice behaviours assessing student acculturation (O’Bryon and Rogers, 2010).

The composition of test norms is the second main barrier for appropriate assessment of children learning two languages. Test norms reflect performance of a reference group to which individual children will be compared in order to make a diagnosis (Standards for Educational and Psychological Testing, 1999). Typically, the question asked in order to make a diagnosis of learning difficulties is whether the individual child performs within the range of or significantly below the reference or normative group. If low performance is below that of the reference group for reasons other than underlying ability differences, then the test is not appropriate for that particular child. Although much of the research demonstrates that children learning language in monolingual and bilingual environments demonstrate a high degree of similarity, they also demonstrate some cross-linguistic influences in language performance. Thus, bilingual children do not present profiles of acquisition that are like that of two reference groups (one for each language), particularly in their weaker language (Paradis and Genesee, 1997). The appropriate reference group for a bilingual child is other children learning language in the same or a similar type of bilingual language context.

Consequently, normative data should never be applied to a population, other than that from which it was developed, that is; norms developed from English monolingual speakers should never be applied to bilingual speakers of English (Roseberry-McKibbin 1994). It should be understood that normative patterns for bilingual acquisition will be different (for each language spoken by an individual) when compared with monolingual acquisition patterns for those languages (Quinn 2001). It is widely accepted that bilingual speakers do not form a homogeneous group (Nicoladis and Genesee 1997, Gutierrez-Clellen 1999), yet Stow and Dodd (2004) state that a few researchers presenting normative data for bilingual children rarely comment on whether their sample had a sequential or simultaneous pattern of acquisition. They elaborate by suggesting that where 
authors have attempted to offer bilingual normative data in tandem with monolingual data the quality of that data may vary. For example, The British Picture Vocabulary Scale: Second Edition (Dunn, Dunn, Whetton and Pintilie, 1997), offers normative data developed from 2571 monolingual children over an age range of 3:00-15:08, whilst the normative data offered for bilingual children were developed from 410 bilingual children (who spoke a range of language combinations and about whom the test authors point out there was no information regarding length of attendance at British schools) and only covers the age range 3:00-8:05.

Related to this is how language assessments adapted from English for other language groups are almost always normed on monolingual groups (e.g. adaptation of the Peabody Picture Vocabulary Test to Spanish; Test Vocabulario de Imagenes Peabody [TVIP]) or, bilingual children are tested in only their home language (e.g. PLS-3). The result is that bilingual children are often inappropriately compared to a monolingual norm. Performance of SpanishEnglish bilingual children on the TVIP (Dunn, Padilla, Lugo and Dunn, 1986) illustrates this point. In the development and adaptation process of this test, norms were collected for monolingual Mexican and Puerto Rican children (in Mexico and Puerto Rico). Comparisons of a US bilingual sample with the Mexican and Puerto Rican samples indicated a significantly lowered performance (Dunn, 1987). This lowered performance of the bilingual group is likely because the children knew items in their second language but were not also tested in that language. Evidence of such distributed vocabulary knowledge comes from Umbel, Pearson, Fernandez and Oller (1992), who compared bilingual (SpanishEnglish) first graders' performance on the PPVT-R and the TVIP. Children who were reported to use both languages at home and those who only spoke Spanish at home scored similarly (below the normative mean) on the TVIP. In English, children who spoke English and Spanish at home scored almost one standard deviation below the mean on the PPVT-R. Children who spoke only Spanish at home scored about two standard deviations below the mean for the English version of the test. When they compared performance on the 61 items that the two versions have in common; investigators found that a significant proportion of words (33-41\%) were known only in one language or the other. These results 
imply that the two single language scores likely underestimated children's vocabulary knowledge.

Duncan (1989) argues that there will always be controversy about the role and usefulness of standardised assessment (SA) being used with bilingual children as a child performing in a linguistically contrived context will result in artificial and low performance scores. Additionally, these assessments are often standardised on one population, e.g. white, middle class children and used on other population groups which are socio-linguistically different, e.g. working-class children from urban areas. This indicates that the difficulties in assessment of language impairments and/or other learning difficulties in bilingual children are a lack of standardised (or static) test tools that are valid and reliable for that purpose (Cummins, 1984).

With the indices of successful outcomes for English language learners not consistently documented in schools and (O'Bryon and Rogers, 2010) and as well as the assessment of second-language learners having become a challenge to school psychologists, assessments and intervention practices for second language learners continues to be a timely topic (Mccloskey and Athanasiou, 2000). However, with an absence of a shared consensus among school psychologists with NASP membership as to what constitutes "best practice" methods in the intellectual assessment of linguistically diverse students, there is an acute need for school psychologists, university trainers and members of professional associations "...to assure the appropriation of appropriate school psychological services to all children" (Bainter and Tollefson, 2003, p.602). 


\section{Appendix III}

Alternative Approaches to the study of 'Race', Culture and Ethnicity

As outlined in detail below, although the positivist and critical realist approaches to the study of 'race', culture and ethnicity were initially considered for the current research study, both were rejected in favour of the Social Constructionist approach.

\section{Positivist Approach to Race, Culture and Ethnicity}

Adherents of positivism state that there is one reality which explains causation between variables (Robson, 2002) and that this causation, once reconnoitred, is thought to create a universal causal which is replicable and, therefore, generalisable to the wider population (Robson, 2002). According to this perspective, reality is objective, universal and quantifiable and through the application of science, it can be identified, shared and "seen" (Ashworth, 2003). As the positivistic approach is "...founded on a belief that the study of human behaviour should be conducted in the same way as studies conducted in the natural sciences (Collis and Hussey, 2003, p.52), there is little notion of the person as the perceiver of his or her world and even less thought seems to be afforded to the possibility of the person as a conceiver or constructor of his or her world (Ashworth, 2003). Here, viewing knowledge, even the most common sense everyday aspects, as being derived from and maintained by social interactions, is considered to be open to bias, ungeneralisable and as lacking scientific rigour (Johnson and Christensen, 2010).

When applied to the discourse of 'race', culture and ethnicity, as the key issues being addressed by the current study, positivism postulates that there "...is one reality..." about such matters "... in the social world that is discernible through scientific inquiry by people of any racial background" (Hunter, 2002, p. 130). Here, the application of positivistic principles to the study of human beings, lends credence to the view that people are reactive and passive organisms, determined by their environment in an almost unidirectional way (Gergen, 1999). 
However, Goldberg (1993) stresses the acknowledgement between racial knowledge and power and states that "Power is exercised epistemologically in the dual practices of naming and evaluating. In naming, or refusing to name things in the order of thought, existence is recognised or refused, significance assigned or ignored, beings elevated or rendered invisible" (p. 150). According to Goldberg (1993), epistemologies have the power to create realities that serve the ideological interests of the state or the dominant group. Here, positivist researchers assist in reinforcing the ideologies of the dominant group by using science to avoid excessive discussion and language (Giroux, 1993; Hunter and Nettles, 1999) around race, culture and ethnicity. This view, of using scientific research to serve dominant discourses, is also echoed by West (2002) who states that powerful discourses have the ability to “...produce and prohibit, develop and delimit forms of rationality, scientificity and objectivity which set perimeters and draw boundaries for the intelligibility, availability and legitimacy of certain ideas" (West, 2002, p. 49). According to West (2002), science is powerful and is influential in presenting certain ideas and making others invisible. Accordingly, scientific research, through the epistemology of positivism, makes available the reality of "colour-blindness" and makes invisible "institutionalised racism" (Neubeck and Cazenave, 2001).

As a result of this, positivist methodology has the potential to neutralise- even lessen- the importance of having discussions around the experiences of marginalised groups, whose 'race', culture and ethnicity is different to the identified dominant group, in society. Anderson, Silver and Abramson (1988) note that this takes place through assigning racially matched interviewers to gather data or by racially identifying all participants or data or by being racially "sensitive" and trying to build trust and ensure an "objective" interpretation of otherwise racially situated social facts. Examples include researching marginalised groups in society, based on their racial, ethnic and cultural background and attributing their lack of progress on economic factors (e.g. Wilson, 1978) without considering enduring issues of white privilege and racism (Rosenthal, 1999; Sanchez-Jankowski, 1999; Hunter, 2002). Therefore, the idea that only evidence gathered through the positivist approach is legitimate evidence or knowledge about racial, cultural or ethnic inequality is unsubstantiated. In the positivist method, factors relating to personal 
experiences, history and culture are “... all regarded as potential polluters of true knowledge and are not considered potential providers of knowledge or truth..." (Hunter, 2002, p.130) and that "...the only things that can be "known"...are phenomena that can be operationalised and measured in a satisfactory way" (Hunter, 2002).

As the dominant discourse of science, positivism becomes the taken-for granted norm that is above question and, by extension, not subjected to critique. However, during the last decade, a growing body of psychological research has acknowledged the general inadequacy of positivism in the study of human beings (Hoffman, 1985). It has been argued that "...sciences are not value-free or scientifically objective and never can be" (Ladner, 1973 in Hunter, 2002, p.121) and that "...racism and power are not outside of the research process at all" (Hunter, 2002, p.119). The latter, it has been stated, has the potential to affect nearly every aspect of how researchers conduct their research from the choice of research questions to the interpretation of their data (Andersen, 1993).

For these reasons, positivism was not considered as an appropriate epistemological approach for the current study.

\section{Critical Realist Approach to Race, Culture and Ethnicity}

In contrast, critical realism offers a way forward in allowing one to consider and evaluate the ineffable perspectives and explanations of EPs in relation to their work with individuals from diverse backgrounds. As EPs experiences are most likely to be described as memories, thoughts, emotions and behaviours, the researcher seeks to identify those deeper lying mechanisms which are taken to generate empirical phenomena. Critical realism, which was developed as an alternative to traditional positivistic models of social science (Cresswell, 2009), assists in identifying what these deeper lying factors may be.

Within the Critical Realist framework, reality is said to exist independently of us and our perception and/knowledge of it (Bhaskar, 1998; Harre, 1986). According to Bhaskar (1998), the notion of reality as consisting of three domains- the empirical, the actual and the real- is central within critical realism. The empirical 
domain includes that which we can observe: things that happen and exist according to our immediate experience. The actual domain is a broader one and refers to that which transpires independently of the researcher or any other observer who might record it. Finally, the real domain includes those mechanisms that are productive of different events and other 'surface phenomena'. According to critical realism, the task of science is to explore the realm of the real and how it relates to the other two domains. The empirical domain is narrower and can be seen as a site of expression of the other two domains. Therefore, according to critical realism, the aim of scientific work is “...to investigate and identify relationships and non-relationships, respectively, between what we experience, what actually happens, and the underlying mechanisms that produce the events in the world" (Danermark, Ekstrom, Jakobsen and Karlsson, 2002, p. 21).

It is the interest in mechanisms of a 'deeper dimension', which distinguishes critical realism from other traditions. It shares the interest of positivism in the objective world, patterns, generalisation, and in finding causalities, but it also diverges from this tradition in claiming that the study of the observable is too superficial, as it disregards the unobservable mechanisms that produce the phenomena that positivists seek to measure and explain. It is not possible to reduce the world to observable objects and facts, critical realists argue. Moreover, they do not accept a distinction between theory and observation or the interest in finding all-encompassing laws. Instead critical realism takes an interest in complex networks of theoretical and observable elements characterising efforts going beyond the surface of social phenomena. It shares with a great number of qualitative approaches an interest in synthesis and context, but it also strongly emphasises the objective nature of reality, and it argues that a focus on social constructions is insufficient and misleading.

With this mind, it could be argued that investigating the possible challenges faced by EPs relating to the domains of 'race', culture and ethnicity of pupils in schools is best understood through a critical realist paradigm as the relevance of knowledge is dependent on the nature, power and mechanism of the objective reality faced by the individual EP. The 'real' is central to critical realism, in that there is a strong conviction regarding the real and the possibility of identifying it. 
Something is real if it has a causal effect, that is, if it affects behaviour and makes a difference. Reality does not just consist of material objects. Ideas and discourses are real and can have causal effects. Ideas about, for example, political/economic changes- such as the recession, increased taxes and higher living costs and the negative portrayal of immigration by the media may lead some to view the descendants of immigrants, as an added burden on society, which may subsequently come across in their interactions with this group of individuals. These ideas are real in the sense that they exist and work as mechanisms with causal effects. It is possible to identify at least four different types of realities; material, ideational, artefactual and social. A given entity can consist of several of these realities.

Critical realism examines the different mechanisms which have implications in terms of different effects and events, the forces and characteristics that mechanisms produce, and the intricate connections between different structural levels, that contribute to the complexity of causal forces, and that make possible the treatment of these as single, isolated factors. Therefore, it could be argued that a White EP may find working with a pupil from Black, or minority ethnic (BME) background difficult; as the psychology they have been trained in, propagates Western beliefs about what makes a good pupil, namely someone who is autonomous and individualistic and competitive. However, this may contrast sharply with the non-Western culture of the BME pupil, who may live by a philosophy where interdependence, a hierarchical familial, social and community structure with a strong belief in religious or spiritual forces are the shapers of one's destiny (Owusu-Bempah and Howitt, 2000). According to Bhaskar (1998), causality should thus not be understood in terms of universal, predictable patterns, but rather as contextual and emergent, in changeable societies. According to critical realists, social reality is often slow in changing, but still emergent and varied as a consequence of the different processes that are part of producing it. As part of the project of accounting for typical patterns, while avoiding the misconception of statistical regularity and predictability, critical realists sometimes use the expression semi-regularity, which indicates "...the occasional, but less than universal, actualisation of a mechanism or tendency, over [a] definite region of time-space" (Bhaskar and Lawson, 1998, p.13). 
Perhaps, most importantly, critical realism, in a Marxist emancipatory tradition, is concerned with social systems that are constructed by ideas, discourses, gender, 'race', class and other social institutions (Denzin and Lincoln, 2003). Critical realism is concerned with the way those issues of power and justice affect knowledge (Kincheloe and McLaren, 2003; Fleetwood, 2005). Kincheloe and McLaren (2003) point to a 'consensus' emerging amongst critical realists that power can be used both oppressively and productively (p.439). An important aspect of the productive use of power is 'empowerment', developing a 'critical democracy', i.e. one where marginalised people and communities can interject with confidence and influence (Fleetwood, 2005). Critical realism holds that "...we will only be able to understand- and so change- the social world if we identify the factors, or mechanisms, at work that generate those events and discourses" (Bhaskar, 1998, p.89).

Although Critical Realism offers an enhanced understanding of causal forces in social affairs (Steele, 2005) than Positivism, e.g. taking into account the observations, feelings and behaviours of the researcher and the ways in which these are likely (or not) to impact upon the delivery and outcomes of the research, it was rejected as an epistemological approach for the current study. It was felt that Critical Realism, while embracing an interpretive axiology, ontology and epistemology that probes "...contextual nuances as interpreted by ourselves in concert with others" (Piantandia, Tananis and Grubs 2004 p. 335), aims not just to reveal the underlying structures and mechanisms "...that produce the events in the world" (Danermark, Ekström, Jakobsen and Karlsson, 2002, p.21); it also helps to fulfil the emancipatory potential of social research (Robson, 2002). In this regard; critical Realism, according to Alvesson and Skoldberg (2009), has a "...radical vein: what is important is not just to explain the world but also to change it" (p.39). Since the area under study relates to the challenges experienced by EPs when working within a multicultural context, the focus of the current study is not to explain how EPs work with those from a diverse racial, cultural or ethnic background, nor how to change their ways of working with this population; the focus, due to a dearth of research in this area of study, is to initiate dialogue, interest and understanding rather than interpreting their views and creating a single reality from the researcher's perspective. 
In addition, despite critical realism opposing Positivism and recognising that social science typically operates within open rather than closed systems, it has been argued that critical realism demonstrates a significant inclination towards arranging the social world in detached and rigid categories which appear to be heavily "....inspired by the world of physics" (Alvesson and Skoldberg, 2009, p.48). For example, critical realism's reliance on structure, forces and mechanism as its most dominant ideas, are concepts that are analogous to those of natural science. As a result of this, when these ideas leave "...the realm of natural science and enters that of the social, the vocabulary of forces and mechanism does not work quite as well." Represented thus; "...social phenomena come across as mechanical and often they run the risk of being overly simplified. They do not work quite as well in trying to explain complex matters..." (Alvesson and Skoldberg, 2009, p.48). For these reasons, critical realism was considered an inappropriate epistemological framework for the current study as it has been described as a providing an "ambiguous view of the world" when trying to explain what is taking place in the world (Alvesson and Skoldberg, 2009 p.47). This is in sharp contrast to the purpose of the current study which aims to ascertain the views of EPs in relation to the diversity of the population they serve; views which are likely to entail complex and emotive elements that would reveal a profound insight into their unique experiences.

While it is accepted that using the social constructionist approach leaves the research open to interpretation, the validity of the final themes identified in the data were thoroughly considered through the use of critical conversations with an experienced researcher and supervisor. In addition, the researcher immersed oneself in the data, with transcripts from the focus groups and semi-structured interviews read several times to ensure accuracy and to certify whether the data selected represented a true reflection of the EPs experiences. Furthermore, validity was ensured through the use of the EPs own words (Patton 2002). 


\section{Appendix IV}

\section{Email Principal Educational Psychologists and/or Service Managers}

Dear [Principal Educational Psychologist/Service Manager],

I am currently enrolled on the Professional Doctorate in Educational and Child Psychology course at the University of East London, headed by Programme Director, Dr Mark Fox. The purpose of this email is to request your permission to contact the Educational Psychologists (EPs) employed within the [name of Educational Psychology Service (EPS)] of the London Borough of [Name] and invite them to participate in my doctoral research project.

The changing population of public schools demands that EPs have the ability to work with diverse students, parents and teachers. With this in mind, the aim of my project is to explore the experiences of EPs working in culturally, racially and ethnically diverse settings and to further determine how well, if at all, their training courses prepared them in working with pupils from culturally, ethnically and racially diverse, i.e. Black, minority ethnic (BME), backgrounds. As well as providing a research-based framework to explain how EPs deal with the dynamics of 'race', culture and ethnicity when working with pupils from diverse backgrounds, it is hoped that this study will have important implications on educational psychology training courses in the UK in encouraging course leaders to design relevant and appropriate curricular and training experiences. Finally, in addition to providing an overarching goal to guide training and practice, it is hoped that the research findings provide insight into the specific strategies and qualities that should be cultivated by training courses and those in practice who are looking to increase their skills.

I am hoping to recruit 15 individuals from the [EPS] who are experienced in providing services to diverse students, parents and teachers in the role of an EP within the London Borough of [name].

Prior to recruitment I will have gained approval from the University of East London's University Research Ethics Committee and subsequently be closely 
supervised by Dr Tina Rae; Academic and Professional Tutor, at the University of East London.

All participants will be asked to contribute to a focus group to speak freely about this potentially sensitive topic. This will be followed by interviews which will be conducted using a semi-structured interview schedule, lasting approximately 1$1 \frac{1}{2}$ hours in length at a suitable location for both participants and the researcher. Although audio-recordings will be made of participants' responses and notes takes during the sessions, participants' identities will remain confidential with names and other information that may render participants identifiable will be removed from the write-up of the study and replaced with a pseudonym or arbitrary initials. Confidentiality may only be breached in exceptional circumstances in accordance with the British Psychological Society's Code of Ethics and Conduct (2009), where '...there appears sufficient evidence to raise serious concern about (a) the safety of clients; (b) the safety of other persons who may be endangered by the client's behaviour; or (c) the health, welfare or safety of children or vulnerable adults' (vi, p.11). In this case, my project supervisor (Dr Tina Rae) will be contacted to discuss any possible concerns, unless the delay would involve a significant risk to life or health.

Upon gaining your consent, I will be happy to email you a preliminary contact email which may be forwarded to prospective participants for their deliberation via email.

In the meantime, should you have any questions, please do not hesitate to contact me.

Thank you for your time.

Kind regards,

Musrat Ashraf 


\section{Trainee Educational Psychologist}

University of East London

Stratford Campus

Water Lane

Stratford

London

E15 4LZ

Email: [studentID]@uel.ac.uk

\section{Supervisor}

Dr T. M. Rae

Academic and Professional Tutor

School of Psychology

University of East London

Stratford Campus

Water Lane

Stratford

London

E15 4LZ

Telephone: 02082234097

Email: t.m.rae@uel.ac.uk 


\section{Appendix V}

\section{Research Study Information for Participants}

Study Title: The Impact of 'Race', Culture and Ethnicity on the Practice of British Educational Psychologists.

Dear (Participant),

You are invited to take part in a research study.

It is important that you understand aims of the research and what your participation will entail before you make a decision. In order to make an informed decision about whether or not you would like to take part in this research study, please consider the following information carefully and do not hesitate to ask any questions before deciding whether you would like to participate or not.

\section{Purpose of the Study}

The aim of this research is to explore the experiences of educational psychologists working in culturally, racially and ethnically diverse settings and to further determine how well, if at all, their training courses prepared them in working with pupils from culturally, ethnically and racially diverse, i.e. Black, minority ethnic (BME), backgrounds. In addition, as well as providing a researchbased framework to explain how educational psychologists' deal with the dynamics of 'race', culture and ethnicity when working with pupils from diverse backgrounds, it is hoped that this study will have important implications on educational psychology training courses in the UK in encouraging course leaders to design relevant and appropriate curricular and training experiences. Finally, as well as providing an overarching goal to guide training and practice, it is hoped that the research findings provide insight into the specific strategies and qualities that should be cultivated by training courses and those in practice who are looking to increase their skills. 


\section{Invitation to Participate}

The study is looking to specifically recruit educational psychologists and explore their experiences of working with a diverse cultural, ethnic and racial population, educational psychologists within diverse London boroughs. Since the Simpson's Diversity Index (SDI) score of the 2011 Census has identified your borough as belonging to the top-thirty most diverse local authorities in London (2011 Census, Office for National Statistics), Educational Psychologists in the borough have been invited to take part in the current study as it is believed that they would have had more opportunities in interacting with and providing services to pupils, parents and teachers from diverse backgrounds.

In addition, [Name of Principal Educational Psychologist/Service Manager] has consented to educational psychologists within [his/her] service being involved with this project, should they wish and, you have expressed an interest in my project via contact by email or by telephone to [Name of Principal Educational Psychologist/Service Manager].

\section{Consent to Participate}

The decision to participate in this study is entirely yours. If you decide that you would like to participate in this research and contribute to unique discussion around the impact of 'race', culture and ethnicity on the practice of British Educational Psychologists, you will be required to sign a consent form that indicates your informed willingness to engage in this research study. If you do decide to participate in this research study, you will be free to withdraw from the study at any point up until your responses have been transcribed, without giving a reason.

\section{Research Procedure}

Participation in this study, requires individuals to contribute to a focus group to speak freely about their views and experiences of working in culturally, racially and ethnically diverse settings and to further determine how well prepared they felt after graduation from their training courses of working with pupils from 
culturally, ethnically and racially diverse, i.e. Black, minority ethnic (BME), backgrounds. As this is a potentially sensitive topic, the focus group discussions will be followed by interviews which will be conducted using a semi-structured interview schedule, lasting approximately 1-1 1/2 hours in length, which will further explore how educational psychologists' deal with the dynamics of 'race', culture and ethnicity when working with pupils/families from diverse backgrounds. Audio-recordings will be made of participants' responses and notes takes during the sessions. Participants' identities will remain confidential with names and other information which may compromise the participants' identity removed from the write-up of the study and replaced with a pseudonym or arbitrary initials.

\section{Possible Advantages and Disadvantages of Participation}

It is hoped that this study will allow you the time and opportunity to reflect on your experiences of being an EP working with those from BME backgrounds and to discuss what challenges, if any, you may face in your practise in a nonjudgemental environment. It is also hoped that this study will have important implications on educational psychology training courses in the UK in encouraging course leaders to design relevant and appropriate curricular and training experiences. Finally, in addition to providing an overarching goal to guide training and practice, it is hoped that the research findings provide insight into the specific strategies and qualities that should be cultivated by training courses and those in practice who are looking to increase their skills.

Discussing 'race', culture and ethnicity are sensitive and, often, controversial concepts and it may be that sharing your experiences may be a difficult. If you find that you find some questions too distressing, you may choose not to answer them.

\section{Concerns about this Research Study}

If you have any concerns about this research, it would be helpful if you could highlight these to me, in the first instance, in person or via telephone or email (details provided below), so that I can do my utmost to ensure any questions, 
concerns or queries are addressed fully. If you remain concerned and wish to express your views formally, you can do so by contacting my research Supervisor, Dr Tina Rae (please refer to the contact details provided below).

\section{Confidentiality}

Responses from the focus groups and subsequent semi-structured interviews will be kept strictly confidential throughout the study. All names and information which may potentially result in a participant being identified will be kept securely. Others in your services will not have access to any raw research data which may be able to identify you at any time. Anonymised data collected during this research may be shared with my research supervisor at the University of East London and professional assessment bodies- all with a duty of confidentiality- in order to evaluate the quality of this doctoral research project. All audio recordings of participants' responses will be destroyed once my doctoral degree, of which this research study is a required, has been conferred. Anonymised data will be kept for 5 years post research project submission (June 2019) in accordance with the University of East London's University Research Ethics Committee guidelines after which, it will be destroyed by the researcher.

Due to the time constraints of doctoral research, the use of an appropriate transcription service may be used to transcribe your interview. Prior to this, the service will be required to sign a non-disclosure and confidentiality agreement and any recordings will be labelled either using letters, i.e. A, B, C or numbers, i.e. $1,2,3$, to protect participants identity. These findings may be shared via academic or professional presentations and participants will not be identified in any report or publication. Any quotes used will be fully anonymised. This research has been reviewed and approved by the University of East London's University Research Ethics Committee to protect your safety, rights, dignity and well-being and no objections have been raised on ethical grounds.

Please note that although the utmost confidentiality will be ensured with participants responses, confidentiality may be breached in exceptional circumstances in accordance with the British Psychological Society's Code of Ethics and Conduct (2009), where '...there appears sufficient evidence to raise 
serious concern about (a) the safety of clients; (b) the safety of other persons who may be endangered by the client's behaviour; or (c) the health, welfare or safety of children or vulnerable adults' (vi, p.11). In this case, the project supervisor (Dr Tina Rae) will be contacted to discuss any possible concerns, unless the delay would involve a significant risk to life or health.

\section{Further Information}

The aim of information above is to provide you with an overview of the current research study and to allow you to consider whether or not you would like to contribute to the unique study on the impact of 'race', culture and ethnicity on the practice of British EPs. If you have any further questions which you would like addressed, please do not hesitate to contact me or my research supervisor on the contact number/email provided below.

If you are interested in participating in this study please fill in the consent form below this information sheet and return it to me via the email address provided.

Thank you for your time.

Kind Regards,

Musrat Ashraf 


\section{Trainee Educational Psychologist}

University of East London

Stratford Campus

Water Lane

Stratford

London

E15 4LZ

Email: [studentID]@uel.ac.uk

\section{Supervisor}

Dr T. M. Rae

Academic and Professional Tutor

School of Psychology

University of East London

Stratford Campus

Water Lane

Stratford

London

E15 4LZ

Telephone: 02082234097

Email: t.m.rae@uel.ac.uk 


\section{Appendix VI}

\section{Consent Form}

Study Title: The Impact of 'Race', Culture and Ethnicity on the Practice of British Educational Psychologists

Name of Researcher: Musrat Ashraf, Trainee Educational Psychology

Participant's Declaration: I confirm that having considered the information provided, I agree to take part in the study described above. I understand that I will be free to withdraw from the study at any point up until my responses have been transcribed, without giving a reason. I also agree to anonymised quotes from my interview may be used in any publications or presentations of further academic study.

Signature:

Name:

EP within the London Borough of:

Date: 


\section{Appendix VII}

\section{Participant Debrief}

1. Recap on purpose of study

2. Review of interview

- How did you find the interview?

- What stood out most of all for you during the interview?

- Are you happy with what was asked and how you responded?

- Is there anything that worries you about what was said by the researcher or perhaps, yourself?

- Do you feel like you said what you had to say?

- Will you be okay after this study?

- Do you have any further questions that you would like to ask?

3. Next steps

- Email address provided if the participant has any questions or concerns post study 


\section{Appendix VIII}

\section{Questioning Route}

\section{Diversity}

In what ways have you seen issues of race, culture and ethnicity emerge in your line of work as an EP?

How do you feel when you come across a CYP or parents who are very different to yourself, in terms of RCE?

How comfortable do you feel addressing certain issues with parents when you do feel like it's a cultural issue?

Does being the same ethnicity as the client give you richer information? Why?

Does being the same ethnicity or from the same culture as CYP and the families make your job easier? How? Why?

How do you establish trust when you have a very defensive BME parent who does not want to engage, perhaps as a result of them or their child being labelled/stereotyped?

\section{Schools}

Have you ever come across school teachers or SENCos who have made sweeping judgements or statements about a group of children or their families? If so, how did you deal with those?

How do you promote equality of access? What do EPs need to do? 


\section{Language}

How have you dealt with instances where you have tried to interact with a parent and they speak a different language to you and an interpreter has not been present?

How have you felt when parents haven't been able to speak English and communicate effectively with you?

How would you assess a child who has language delays if it is an assessment heavy borough?

How easy is it to disseminate assessment information that you've collected from the CYB to their parents if their parents have limited English? What do you do?

\section{Role}

Have any of your ever felt that your RCE has been a barrier to your interaction with parents or CYPs? If so, how?

What role do we have as educational psychologists in empowering BME parents and CYP in light of the injustices they face at school? Or do we have a role?

What roles do EPs have in meeting the needs of significantly underachieving BME boys?

Do EPs have a responsibility to challenge and neutralise the zeitgeist in schools and how it's coming across towards parents and children that might be from the background being vilified in the media? If so, how?

\section{Self}

Have there ever been times when your own RCE has affected your experience of working with parents, teachers, children and young people? How does it come 
across being Caucasian when working with parents who are not from your own culture/ethnic backgrounds?

When you first get a case, how does your own culture/ethnicity play a role in that?

\section{Training}

Where did you complete your training?

Do you feel that the curriculum of the course prepared you to work with a diverse range of people? How?

What stood out re: diversity that you took with you into your practise?

Do you feel EP training courses are fine as they are re: diversity? Why? 


\section{Appendix IX}

\section{Terminology}

'Race'

The term 'race' refers to the notion that humans share distinct, inherited and permanent biological characteristics, such as skin colour, hair texture, facial features and stature (d'Ardenne and Mahatani, 1999, Barkan, 2012). However, with a lack of evidence of 'race' belonging to a biological category:

With the vast expansion of scientific knowledge in this century, however, it has become clear that human populations are not unambiguous, clearly demarcated, biologically distinct groups. Evidence from the analysis of genetics (e.g., DNA) indicates that most physical variation, about 94\%, lies within so-called racial groups. Conventional geographic "racial" groupings differ from one another only in about $6 \%$ of their genes. This means that there is greater variation within "racial" groups than between them...

...Physical variations in any given trait tend to occur gradually rather than abruptly over geographic areas. And because physical traits are inherited independently of one another, knowing the range of one trait does not predict the presence of others. For example, skin color varies largely from light in the temperate areas in the north to dark in the tropical areas in the south; its intensity is not related to nose shape or hair texture. Dark skin may be associated with frizzy or kinky hair or curly or wavy or straight hair, all of which are found among different indigenous peoples in tropical regions. These facts render any attempt to establish lines of division among biological populations both arbitrary and subjective.

(American Anthropological Association (American Anthropological Association

Some have felt it necessary to put the word 'race' in inverted commas to highlight that 'race' reflects the social construction; historical and locally prescribed ways of thinking, seeing and talking, which are rooted in the racist history of colonialism, slavery and apartheid (Durrheim, Hooks and Riggs, 2009). 


\section{Ethnicity}

Ethnicity refers to the mutual social, cultural, and historical practices, stemming from common national or regional backgrounds that make sub-groups of a population different from one another. Equally, an ethnic group is a sub-group of a population with established and shared social, cultural, and historic experiences; with relatively distinctive beliefs, values, and behaviours; and with some sense of identity of belonging to the subgroup (Fernando, 2002). Perceived thus, the terms ethnicity and ethnic group avoid the biological inferences of the terms race and racial group (Jenkins, 1986; Barkan, 2012). However, similar to 'race', the importance attached to ethnicity illustrates that it, too, is in many ways a social construction, and that one's ethnic membership has important consequences for how one is treated (Barkan, 2012). In particular, history and current practice indicate that it is easy to become prejudiced against people with different ethnicities from our own, e.g. the horrors of Nazism, ISIS, ethniccleansing and civil wars raging across the world.

\section{Culture}

While ethnicity is more concerned with group identity, culture is more about group practice, values and beliefs (Schein, 1990). However, like ethnicity, culture is thought to be continuously shifting and evolving and is thus, notoriously difficult to define (Spencer-Oatey, 2012). However, Ferraro (1998) argues that culture is associated with social groups, in that "culture is shared by at least two or more people, and of course real, live societies are always larger than that. There is, in

other words, no such thing as the culture of a hermit. If a solitary individual thinks and behaves in a certain way, that thought or action is idiosyncratic, not cultural. For an idea, a thing, or a behavior to be considered cultural, it must be shared by some type of social group or society" (Ferraro, 1998, 16).

Given this definition, as almost everyone belongs to a number of different groups and categories of people at the same time, people unavoidably carry several layers of mental programming within themselves, corresponding to different levels of culture, e.g. regional, ethnic, religious, gender, social class and/or linguistic affiliations (Hofstede 1991), which suggests that everyone is 
simultaneously a member of several different cultural groups and thus could be said to have multicultural membership (Spencer-Oatey, 2012).

\section{Black, Minority Ethnic (BME)}

The term 'Black' has political connotations and refers to those with African and Caribbean origins, who have a shared history of European colonialism, neocolonialism, imperialism, ethnocentrism and racism (British Sociological Association [BSA], 2005). In addition, the Commission for Racial Equality (2007) indicates that the term 'ethnic minority' refers to individuals who, according to the ethnic monitoring categories used in the 2001 National Census, selected an ethnic category other than 'White'. However, it has been argued that the use of 'ethnic minority' overlooks or ignores the ethnicity of majority groups (BSA, 2005) and that 'minority ethnic' as a term should be used instead as it places the emphasis on the minority status rather than the ethnicity, whereas ethnic minority places the emphasis on the minority status of the group.

\section{Diversity and Multiculturalism}

According to Ortiz and Flanagan (2002) diversity denotes “...any individual or group whose background and experiences differ significantly from that reflected by the mainstream" (p. 339), while the definition of multiculturalism is complex and is often contingent upon the context in which it is discussed (Da Silva Rodriguez, 2009), for the purposes of this study, multiculturalism/multicultural will relate to the presence of several cultural, 'racial' or ethnic groups within a society.

\section{White}

The term White refers to individuals with European ancestral origins who identify, or are identified, as White. The term is serves to distinguish these groups from those groups with visible differences in skin colour (e.g. Black, Asian, etc.), and hence derives from the concept of race but is used as an indicator of ethnicity (Bhopal, 2004). Individuals who are white have also been argued to belong to an 
unnamed, dominant but usually unquestioned cultural space that is neutral and normative (BSA, 2005). 


\section{Appendix X}

\section{Example of a Verbatim Focus Group Transcription}

MA: ... So, just to start us off then, in what ways have you seen issues of race and ethnicity emerge in your line of work as an EP? So, just again, have conversation with each other and we'll just kind of explore key themes that emerge...

[Giggling from an EP]

DC: Such a huge issue!

[Inaudible]

$\mathrm{KP}$ : So just issues of...of...is it issues of?

MA: Issues of race, culture and ethnicity that might have emerged- this could have been a comment from the school SENCo, a colleague here or professionals you may have come across.

AHR: Okay, I was at an MPPM yesterday and, erm... the SENCo was going through her caseload and three of the cases she wanted to discuss with us were quite similar, erm, and they, they, they all involved... children, erm, she wasn't actually...sh...she didn't mention exactly where they were all from but two of them were from, er, from Somali families. And, erm... they had behavioural issues and she felt that the parents were not...erm..., they're not taking this issue seriously, that the teachers were bringing up. So she was asking...the MPPM group whether anybody had comes across these issues... before. Whether thishow did other schools deal with this, et...cetera. And, that prompted a discussion from the other people in the, other professionals. Erm, and they were...not sure. It was a lot...people were quite tentative about how they were trying to, to , explain their views about it.

MA: Mmhmm 
AHR: Erm...but she, yeah, she just asked the question because she hadn't come across this before and she wanted to know whether there's any...anything out there. So, this conversation started but...it was...there wasn't anything other than ppeople's, you know, experiences, so no one was able to say...erm, I dunno, to back it up with anything that they knew but that was the conversation that was had and, erm... so, I guess that was about... whether these issues were part of their culture or whether they were not. And... that...

MA: So was she trying to generate an honest...honest, open discussion about whether...

AHR: She was asking a genuine question...

MA:Okay...

AHR: ... because she saw a pattern and she didn't know whether it was a pattern or whether she was making a pa... you know, drawing...conclusions that were there and she, she, she accepted...actually, to put it better into context, she's from up north and she's just recently come to work in London for the first time, erm, so she doesn't know anything about Hounslow really yet.

MA: $\mathrm{MmHmm}$

AHR: Erm, two months into the job and about the other schools I Hounslow. So, she was g'genuinely asking a...

NA: Very naïve question, yeah

AHR: Yeah because she's just hasn't got, had that experience here... to, to base her [unintelligible], you know, experiences

DC: Was that, was that those sort of ver...quite stereotypical views and, and widely held views about Somali families in the borough that prompted me to do that, f...to, to look at that issue for my own research for the doctorate. Erm, in so many schools, I have exactly the same thing- the same patterns about language 
use and not having toys, not playing with children, all those kind of issues and I...felt that the voice of the Somali parents, particularly Somali mothers... would actually struck me often as doing, working really hard and being absolutely [laughs] erm, you know, committed to their kids- there was a real kind of disjudge there-that's what's I wanted to explore, which is exactly that. If, it's one group in Hounslow I think... where there is... a commonly held view, isn't there, I mean, I don't know whether it's true about other... minority ethnic groups, if, if you've experienced that. But certainly for me, that's the group where that, seems to be that real concern, erm...

MA: Okay, and is it, is it concern mostly of a behavioural nature or are there other things happening?

DC: Yeah, but it's also based on the fact that there's lots of Somali, I mean the exclusion rates of...

MA: Yes?

DC: ...Somali boys in secondary school are very high and there are high numbers in Feltham Young Offenders, so there is, there's a whole kind of, there's a...

KP: Hmmm...

DC: ... whole reality there for that but there's, but...

$\mathrm{KP}:$ But the play issues as well...

DC: I really... [drowned out]

KP: ... in the Portage Service...

DC: Yeah...

KP: ...we had, erm, some Somali families, erm, who, who had children with Downs Syndrome and I remember one of the Portage Home Visitors going around and, and taking... I went round for a review and, and her saying that it, it 
seemed to be the, the Somali families didn't really sit down and play with their children in the same way that, erm... maybe other people were encouraged to play with their children. It just wasn't, she felt, it wasn't part of their culture.

DC: Hmm.

KP: So, for this particular little boy, who had Downs Syndrome where she was going round into the home and trying to get the mother to play more, to play more str, in a more structured way, she said it was really, really hard because it just wasn't part of their... it was a lovely mother. She was a really lovely lady but just sit down and play with her son, was something that she just, she didn't... and she had three or four other children, they were older, this was the last one and erm, she said it was part of the Somali culture and I hadn't really heard that before but, I remember thinking "Oh, that's, that's interesting!"

DC: But did it beg a question that, that...

$\mathrm{KP}:$ [clears throat]

DC: ...the way we work as EPs, whether we articulate it, whether we're conscious of it or not, we work within very dominant models of what's good parenting, what's appropriate for, for people to do with regards to their children's education? They are quite narrow models and so other communities who don't have those, whether it's to do with social class or ethnicity, don't fit into that, they're found wanting and I think even with the best will in the world, and we collude in that. I think schools will very often say "Oh they're not doing so and so, they're not interested, they're not following through on this, they're in denial" but it behoves us to try and step back that little bit and think about what, what's our role in that? Should, should we be challenging that? What should we be doing? $\mathrm{KP}: \mathrm{Hmmm}$.

DC: I don't have the answers to that but I think we need to be aware of this. 
MA: Okay, have you ever come across any of that $A$ ?

AHR: ...

MA: With another ethnic minority group?

AHR: Quite often... I agree with D like to what extent... I question I collude with this by not just really saying anything and having come from Wales, where obviously, it's very...erm, kind of...it's predominantly white where I come from... MA: Yeah...

AHR: ... and, erm, not much diversity and to come into Hounslow, I suppose it's, it's been a learning curve for me, in terms of diversity and ethnicity and...it's something that I'm quite comfortable with. However, when I'm sat in a meeting with a SENCo, for example, and, and they are talking about "Oh, this parent's reluctant, erm, this parent's reluctant to acknowledge and, you know... quite often you get this with Asian parents where they're... and they make these broad...stereotypes....and... I have seen that a number of times where parents from certain ethnic backgrounds are reluctant to...t...t...to be... to share their viewpoints with the school. However, er, that might be the same for like kind of white, British parents, we... don't know whether it's just a stereotype...

DC: Hmmm

AHR: ...that a lot of schools, I don't...

MA: So, looking beyond the colour then? Looking beyond...

AHR: Yeah...

MA: ...the ethnicity itself...

AHR: Yeah

MA: ... and looking at it from a wider perspective.

KP: I had... one situation with an MPPM, which, when, when I think round about that time, you'd talked on your first talk about it and it, it really made me think 
about that a little bit. We were at an MPPM and there was a family, there's a child at that school where his father had been very ill for a long time and had died. And they were a Muslim family. And, so, the, the, mother had two sons; one who was about 18 and one who was young, who's about 7. And, erm, it was in September we had the MPPM or October time, last year, and, erm, the father had died, erm, the year before...the... six months before. And the, the school bought it up and said they're really concerned about the boy and about the mother because, erm...she didn't seem to have be, have told her son that his father had died. And, there was this long discussion and of course, in this group sitting round this table, there was the learning support, behaviour support...erm, possibly an EWO...erm...and, er, erm... a family support. We were all white... and there was this discussion about whether, you know, what's the, what, wh...

: How...

$\mathrm{KP}:$ In a Muslim... in a, in $\mathrm{a}$, in the Muslim religion... what happens around death? You know, is it normal not to tell a child?

NA: Hmmm

KP: And, no, nobody really knew.

NA: Hmmm

KP: And, so everyone was so, somebody said "Why don't you, um, you know, is there a mosque round here? Why don't, why doesn't someone speak to the person at the mosque and find out?"

DC: [laughing]

KP: And we just didn't know. Nobody really...

DC: [laughing] No, no!

KP: ...knew really knew! And, it was actually, it was a bit ridiculous really because... [sighs] you know the fact...in, in the end, I saw the child in the end 
and actually he's sort of Aspergers-y... I spoke to the mother, the mother was very upset, she said she had told him now that his father had died but actually he'd just gone "Oh, okay, when's he coming back?"

NA: [laughs]

$\mathrm{KP}$ : Erm, but, but there was this massive issue around, you know, "They're Muslim and what do we do, how do we...?"

AHR: I completely...I had a very similar experience this week and I talked at length to it about, about it with T, who's obviously from a Muslim background herself and, I was so interesting because I said, 'cos I was proofreading someone's report and a child had said within the assessment something about, erm....all Christians should go to hell and that his name means this and it's the sword that cuts people in hell and it was all... quite worrying stuff that he was saying...

[laughing]

AHR: But at the...for, for me, I read, I was proof reading and I went "Oh Gosh!" [laughter]

AHR: Where has this come from and apparently, he said oh, he'd be taught, told this when he went to Pakistan. And, I was like being all "Alright, okay. How did I feel about this, what does this mean?" T read it... and was furious! Absolutely furious! And, she was like this [bangs on table] is not acceptable! You cannot, this child cannot... and, but what, what we did on a side-note, what we did find out that is, his name actually does mean that. And, then we were like "oh" construing it through our own minds like "Oh gosh, how is, that's violent what he's saying but his name did mean sword whether it meant sword to kill...

DC: Did T think it, why did she, how did she differ from what you were thinking? She was thinking... 
AHR: Because I was tentative around it...

[both talk at once]

DC: Yeah... yeah... yeah.

AHR: I wasn't going round saying...T was saying the mosque are answerable to this, who? We need to get the parents in, we need to talk to them. What are the parents doing about this? Are the paren... and she was very passionate about it and I said "Do you know, I feel there is a real...erm, kind of... there was a real opportunity missed in a way that...

DC: $H m m m \ldots$.

AHR: ...that T could have been, she's got an interest in it. There could be a specialism there for her...

DC: Yeah

AHR: ....in terms of...

KP: Yeah

AHR: ...cultural diversity because it's something I can't offer...

DC: Hmmm

AHR: ...because I probably will tip-toe around issues like that because I am not Muslim, I don't come from a Muslim background and I don't know all the, kind of, th-the implications of that...

NA: Ah! But you see...

KP: Yeah...

NA: I...

KP: Yeah, that's...

NA: ...I was having this conversation with $\mathrm{T}$ just this morning, and she- there was erm, the BPS put on a, a, a session recently about race and ethnicity...

MA: Yeah 
NA: Did you go?

MA: I didn't actually. I couldn't go, I was at university.

NA: Oh, um, and she went to that and um, you know, she was remarking on, on the population that, that were there and, wh-when I first started working in Hounslow, I was the only ethnic minority EP here. Erm...and so, this is, this is what we're working with and...

AHR: Mmhmm...

NA: ....and it's a shame that you feel you have to tread on eggshells around it because...we're not going to suddenly change the population of EPs, this is who we are and you should be able to, erm, you know, feel confident to discuss these issues-

AHR: I should, I should!

NA: ... without, erm...

AHR: I felt comfortable to discuss it with my colleague T...

NA: ... because it then becomes...

AHR: ...but...

NA: ...an issue for the ethnic minorities when it's not, it's an issue for people working with this min-

KP: But I felt the same as $A$ to a certain extent that I-I-I-I sensed that, you know when people talk and also because I'm very aware that just because I'm white British doesn't mean that I'm necessarily Christian. And, if somebody...

NA: Hmmm.

KP: ...presumes that's my, who I am...

NA: Hmmm. 
$\mathrm{KP}$ : ...I would feel very wrong presuming that just because...S and $\mathrm{T}$ and you [MA] may be a Muslim that you all have the same views because I know that I don't and when you...

NA: Hmmm.

$\mathrm{KP}:$...talked at the beginning about your experience of going in to this home and just because you may be a Muslim or similar colour to those people that you'd be able to talk to them or understand what they... you know, I'm very aware of that but I see it all as very different, so, although I might talk to $\mathrm{T}$ about something like that, I might think "Well, that's her opinion...

NA: Hmmm.

MA: Exactly.

KP: ...We're all so different,

MA: Yes

KP: ....aren't we? So it's still a very difficult issue.

DC: That's come up in my interviews, the assumption that I must be a Christian... KP: Yes!

DC: ... and I just say well I'm, you know I don't use the word "Atheist"- but I am an Atheist- but, cos I think it kind of feels a bit blunt so I just say "Well, I don't have any faith or something that- in a language the might understand...

MA: Yeah

DC: ... but I agree with what...

MA: Well, I get mistaken- if it helps- for being a Sikh or a Hindu, so...

[laughter]

MA: ... there you are, it just goes to show how it doesn't really mean anything...

DC: Hmmm

MA: ... How you come across, how you look, so... 
KP: Yes!

MA: That's interesting and [turning to A] I like how what you said that you didn't really feel as though you were qualified to kind of intervene...

AHR: I must say, obviously I'm fairly newly qualified and new to the profession and my background, that I'm very reflexive of that, that I don't come from like a very culturally diverse background myself, so l've just started to feel comfortable in a very multicultural area and working with a multicultural population. Erm, not that I would feel uncomfortable but starting to feel I'm not stepping on eggshells all the time...

MA: Hmmm.

AHR: ... so...I think you need to bare that in mind when I'm saying that because I don't know whether other colleagues would- more experienced colleagues who've worked here for a very long time- would feel that way.

KP: Well, no, I felt exactly the same bu-

AMR: Yeah.

KP: -but maybe for different reasons. I also found that having worked for Hounslow for probably because, you know although l've lived in sort of London, outer London all my life, erm, actually working in Hounslow has meant, compared to some of my colleagues, or peers say, my friends, I do mix with a much broader ethnic mix but the social mix are most of the people I know [clears throat] are my friends and, and, it, the, you know the, I suppose as you go through life, you realise that you know when I meet people it doesn't matter what colour they are, what faith they are, you know, I can meet white British people and there's a chasm between us...

DC: Hmmm 
KP: ... and I can meet, you know, people from other countries who've just come over and actually you realise that you're very similar in your views and it doesn't really matter what colour they are, what faith they are, you know that-that...itbut-but-but there is that presumption though I think when we meet people sometimes or when we work with...

DC: But there is...

KP: ...families.

DC: ...but there is a reality, isn't there, there's a reality of the fact that, for example, people from an Afro-Carribean background have been in Britain in large numbers a lot longer and if you just look at youth culture, you'll see the overlap. And, there is a, there's a greater comfort in similarity I think between lots of- I'm just talking about myself- but between white people and...black people [laughs nervously] for that, for that reason because there's been co-existence for a lot longer and sharing of popular culture and those kind of things. I do think for, particularly for Muslim populations, there is difference- I mean, you know, 9/11 mattered- huge Islamophobia. You don't, it's not racism- it's not a you know, I'm stating the obvious obviously but it's not a blanket, in a blanket situation are wethere was a different perception of a Black, a Black Afro-Carribean Black British family, you know, there's a I think great, greater sense of familiarity and common ground perhaps than there is with a Muslim woman who's, who's, you know wearing a veil. I mean I think that you have to acknowledge that...

KP: Hmmm

DC: ... and I think that's an important part of the debate isn't it? And, Hounslow, I think is... different to many other boroughs because it has more of a kind of South-Asian and certain African kind of backgrounds in its residents. 
MA: Have any of you ever felt that your race, culture or ethnicity has ever been a barrier to you interacting with teachers, parents, children/young people?

KP: Yeah, I think, pare- not for teachers, maybe, or children, but I think with parents when I go into parents homes, people who I meet- parents in a, in a oneto-one, I'm a, you know, I'm aware that some parents; I'm very different to them. Particularly if...

MA: How? How are you different?

KP: Well, because I...I suppose because ethically I'm different and when, er, you know, I get a mother and father and the mother sits there, in a her, in a very stereotypical way, doesn't say anything and doesn't speak English, even though she's been here for three or four years and I feel I can't reach her and I can't...hear, hear her views and there's the- the husband who's saying "No!" you know... "Our child doesn't have learning difficulties" or, and, it's a very stereotypical view but sometimes we meet that- I've met that here and I feel like maybe, if I looked like her, if I spoke her language, maybe I could have a better, maybe, better understanding.

DC: Hmmm.

AHR: It's a really important point. I don't think we... language is such an issue here. You can't really...in terms of the work we do, you can't really separate the two often. Erm, that kind of culture, ethnicity, race and language because, again, I was having this conversation with $\mathrm{T}$ the other day about how... I've met many parents now from many different backgrounds, some have always been in the UK, others who have come to the UK, some- they've all got varying degrees of English and I find that... it's really difficult to work productively with someone who speaks very little English. You can do it through a translator but that, that 
connection- you can't read and, and that's another important point like you can't the facial expressions based on their culture might not be similar to ours...

DC: Yes, absolutely.

KP: Hmmm.

AHR: ...so if there's, if it's going through a translator, you can't might not necessarily relete- read how they're interpreting the situation. How they're relating to you as the EP in the situation- it's really difficult. And, er, I had a situation recently where I had a consultation with a Somali mum, who spoke perfect English and that kind of a very strong cultural beliefs but it was such a productive consultation and she was a, kind of, all out for the well-being of her son and she was demanding that we had a group consultation with all of the teachers at the school and I was thinking, would that have happened if DC: Her language...

AHR: ...she didn't speak English?

DC: Uhuh...

AHR: Or to that, if she couldn't couldn't articulate herself to that degree? I think it's an important...

NA: And, not only that but...when I...er, as things have moved along and cuts have been made, erm, the chance of using an actual professional translator... DC: Absolutely.

NA: ... is become less and less frequent and so, you know, when I first started working in Hounslow, I er, er, er, although it was still a bit difficult you know, we, you could more easily find a translator to come and work but these days, it tends to...

DC: Be a TA or somebody.

AHR: Yeah. 
NA: ... and you have no idea whether they have the professional language to explain things like 'statutory assessment' in whichever language they speak or whether they, you know, wha-how they're culturally influencing what they're passing on...

AHR: What their own agenda is if they work with that child as well.

LW: And, I think that's a good point about...

NA: And, the confidentiality if you know that you're talking about quite personal... LW: Yes.

NA: ...things with somebody that they don't necessarily...

LW: I think they often pick someone who is a teaching assistant in the school who speaks that language and then you don't know the relationship when you're discussing confidential issues and hoe it, that will stay confidential. And, if the parent is actually happy to discuss this with that person anyway, so you don't know how much you're actually getting from that.

NA: What l've actually found in relation to that is quite often, because of the barrier between- the language barrier- that person has regularly been translating for the class teacher to, to the parent anyway, so actually, a lot of the time, theythe parent has a relationship with the TA because, the TA's already had to come to the classroom and explain something- which is...quite nice that they already have that rapport and, but it's, it's still not ideal really, is it? AHR: It's not, it's not.

KP: But I've never, in, in, in spite of that, l've never actually, just thinking about it, I've never had a parent ever say to me that they didn't... the only time l've ever had a parent say anything to me was about not having children, I remember once a parent said to me: "You don't have. Do you have children?" And, I had to say no! And they say “Well, you don't know how I feel." And, that's the only- l've never 
had a parent say "Well, you're not a woman, or you're not Somali! Or you're not..." Erm, so they've never said anything even though I might have felt like that it's only about having children... which I disagreed with...

[NA and KP talk at once: Unintelligible: 22:46]

KP: Maybe, maybe...

NA: As a professional

KP: Probably.

DC: It's a [unintelligible] with you, doesn't it?

NA: Yeah

MA: Erm, alright then, when you first get a case then, how does your own culture, your own ethnicity- does that play a role in that? In getting a case and making hypotheses?

AHR: When I was in Wales, it was such a rarity to get a ca- if I got a case and, it was, [laughs nervously] they were from a kind of different...cultural background, it would be a rarity- I think it happened twice in my whole three years of training in Wales!

[laughter]

AHR: It was a box that was ticked in my portfolio! [everyone laughs]

AHR: So, um, yeah, so I did think about it a lot then and it was a big part of my case formulation, I suppose like kind of in terms of my thinking and...

MA: Can you explain that a bit more?

AHR: I can't even think back to the cases now so not really...

MA: Alright.

AHR: Not to do it justice but l'd be like thinking [enthusiastically] "Oh so these are the cultural issues here and we've got different cultures and the school's going 
to be different and- but that is it- it's relevant, I suppose because those children were very much a minority in some of the schools, erm, so it was relevant there. However, here, I don't, don't even half the time like kind of I just go out to the consultation and I...just take it for what it is, I suppose a lot of the time and... KP: Hmmm.

AHR: ... rather than thinking too much into that...

KP: Hmmm. And, I remember when I first trained, well there was that green book about bi- and it was about bilingualism and I remember at, in my training, that was a big, really big issue about cognitive assessments and how you can't use them on bilingual children and how you had to consider those things and it was really important and, of course, when I came to [borough], the first couple of children I looked at, I got the book out, looked at, you know made sure that I made it, thought about their second language. When then every single child I see has English as a second language, it just becomes the norm... Of course that book isn't really relevant anymore. It's so outdated and I'm not sure if they ever really published an update to it.

AHR: Norm

$\mathrm{KP}:$... doesn't it?

DC: Hmmm

AHR: Yeah.

KP: It is no longer a... it's just... and actually in, I remember, well, when I was first in my first one of my first schools was $X$ Infant and Junior schools and in $X$, there're quite good schools and they're mainly in, in the classroom, there'll be one white child in the class and it was almost more, that child's identity within a, within a class that's different to them rather than the other way round and you could guarantee in that school, I always got referred those white children 
because they were the n-, they were often the family's had been moved on from other places and stuck...

DC: $\mathrm{Hmmm}$

$\mathrm{KP}:$...in, in the estate where they didn't want to be because there were problematic families with problematic children and I always got those white boys, were always referred to me in those schools. So it was a completely the reverse, almost!

NA: Hmmm

KP: Because they didn't really identify with the other children. Bu-but that's Hounslow, isn't it! Now, now, everyone...

AHR: And it's true when you're working here just like when you kind of gather background information, when- when did you- were you born in the UK? Moved to the country? What's the, what's the home language spoken...?

NA: Yeah

AMR: What's his grasp of English, what do you think? What's his strongest language? You kind of just go automatically...

$\mathrm{KP}: \mathrm{Hmmm}$

AMR: ...through all of those...

$\mathrm{KP}: \mathrm{Hmmm}$

AMR: ....well, I do anyway...

KP: Yes.

NA: Yeah.

AMR: ... all of that to get- as part of my practice now...

LW: And, I think many of the children that I see were not born in this country...

AMR: Hmmm.

NA: Hmmm 
LW: That's sor-

AMR: Yeah

LW: ...more than $50 \%$ that have come... at some age so they've had prior experience in another country of a different culture, often and, err, and bring with that lots of different things for each...

DC: That's...

LW: ...case.

DC: I think that's right, I was thinking you were talking that maybe to some degree, talking about myself but if I almost, like it's glossed over because so many schools have so many children with, with English as an additional language an' so are good at supporting them, it's almost... by-the-by. And, yet, for some children, I was thinking for the boy I work with, erm, who's Iraqi, who was born there and came here and very difficult behaviour, and a lot of his problem was just his, i-in his head from perhaps from his family. He had this idea in his head and he-and the imagined community, his homeland- all- all lovely, the weather was great, played football all day...

[laughter]

DC: ... and he just couldn't settle because his...

$\mathrm{KP}$ : [inaudible comment]

DC: His heart was elsewhere. Yeah...

[Inaudible comments in agreement]

DC: ... and I think we got that ...

[laughter and inaudible comment]

DC: ... and I think often perhaps we don't acknowledge how significant that is.

AHR: I had, err, an experience of an MPPM once where, um, it was, um, they were talking about a Somali boy who- and they were talking for about 20 minutes 
about the issues this child was presenting with in school, and I asked the question "What's his background?" "Oh, he's moved to this country because his parents were killed in...

DC: [gasps]

AHR: ...Somalia and he's now living with his uncle.

[laughter]

AHR: ... And, I went "Don't you think we should've not started with that 20 minutes ago?!" It was almost like we've got so many of this children who've just have these complex backgrounds in other countries...

TK: Yep!

AHR: Oh, and that, they're here now and so let's just deal with it in the here and now...

TK: Yep.

AHR: ... and perhaps... that's just my experience.

[BRIEF INTERUPTION BY PEP]

DC: Yeah, I think, I mean would- that's something that would dominate on mymy-my experience with Somali women but you kn- over time, some of them have kind of got more trusting and the things that they would disclose- an-you don't have to imagine what they've been through to come here.

TK: Hmmm.

DC: You know and it's just...that even if they don't talk about their children, the trauma!

[Inaudible comment by EP] 
DC: Yeah. Absolutely. And now there is what the Somali community thinks there real more avenues for people to actually, kind of talk about some of that aweful stuff that's happened. But I think you're absolutelty right we-it's because it's almost the air we breathe in Hounslow, isn't it?

[inaudible]

LW: I think that's more of what we're actually dealing with under the surface of all these learning difficulties, I think...

NA: Hmmm

LW:... especially, I suppose I have X- a behaviour school- and erm, often when we're called in to mainstream schools with behaviour, there's all of that trauma hidden...

NA: Hmmm.

LW: ...that's sort of bubbling under the surface...

NA: Hmmm.

LW: ...that I think is with so many children actually.

MA: Yes...

NA: That's a nice way of thinking about it.

KP: Hmmm.

MA: What have your experiences been T?

TA: I'm just thinking, that...all of what you're saying about you kind of come in and $y$ - you've done all the, tsk, the logistical stuff, you know wh-where were they born? What's their first language? D-d-d. And, then I think I,I think I personally stop there. Because...when, when I go there... they may be Asian. They may not be. They may happen to be Muslim. They may- whatever it is. But... I guess I don't wanna assume anything from that...

NA: Hmmm. 
TK: ... because their version of my- of Islam could be completely different to mine. Their version of being Asian could be completely different. They could be Londoners, or... you know! Whatever! It's all...

[laughter]

TK: ... of that, you know! So, I kind of do, make sure that I've- I've checked that and then kinda say right, well, let's just hear what all of this means for them. 'Cos I've gotta kinda problem solve with their...with their understanding of their...situation, I think, so I think maybe I... actively put a hold on it. Tr-to try not to...

DC: Yeah

TK: ...over-identify. Erm, and sometimes like I'm just thinkin' about X [school] where I've just...came from an' my experience there has been... very different because I guess I have X [school]... so actually, most of what I experience here in Hounslow has not been ethnic minority, if I'm honest and it kind of became more ethnic minority when I- when I took on some...

NA: Because $X$ is...?

DC: White, working class.

TK: It's, it's...Yeah...

NA: Right...

TK: ...different kind of, l've had different kind of cases, KP: It's completely different.

TK: Completely different...

KP: Hmmhmm

TK: ...erm, and then... I've- I've had X- the two PD- X [name of specialist setting] Juniors and the Infants PD centre and, as you were saying, I kind of, I didn't realise, I think l'd been so used to kinda X [school] and had, had had the centres 
for quite a while and then did a lesson observation and immediately started to try and look for the white person because...

$\mathrm{KP}: \mathrm{Hmmm}$

DC: [laughs]

TK: ... this must be really difficult...for everybody else here, erm, and I happen to be...working on that particular day with a child that was white, erm, and then when it comes to some of like $X$ [school], if I go back to $X$ [school], the, the families that I've worked with there, I done a lot more TACs and things like that so they're a bit more maybe kind of family- what are the family issues? And, the've j... they've been th- kind of draw to connect with me, "Oh, you know what our Asian women are like?" and they'll look at me...

DC: [laughs]

KP: Yeah

TK: and I'll get that and I'll...and, part of me's like... yeah I can on some level say "Yes, I completely- yeah because they can be this, this, this and this but I still don't know what she's exactly referring to...

NA: Yeah

TK: And, I...and more often than not, it's what I think it is on these particular occasions but it could easily not be.

NA: Hmmm

KP: Hmmm

TK: So, I could be thinking that "Oh, Asian women... really, really good at cooking...

AHR: No! [laughing]

[laughing] 
TK: ... and they're thinking aren't they all...unpleasant women or whatever, so I'll try and double check with them, so where, wherever there's been occasions where they've tried to identify with me because of either, mainly because I'm Asian, l'll try and double check that.

MA: Does that make your job harder?

TK: ...sometimes I guess... in occasions where, th-there's been similarities in language... and they've, th-their English is relatively good but then, there's something's you can't transfer across, y'know if you've got another language, some-something's you can't transfer across. And, even though it's never actually been my language, it's kinda similar, so I'll say, they'll go "Do you speak Hindi?" and I'll go "No" and they'll say something anyway! 'Cos they know l'll maybe be able to get something and then it's because they're trying to...express something and very often thinking about it, it's something... really emotional that they-they just can't say in English and then I'm in a position where then I'm suddenly the translator in the meeting and I'm like "What mum just tried to say to me..." and then I'm tryin' to express it to them and then double check with mum that I've obviously interpreted it right because it's not the same language- and even if it was the same language, it could still be interpreted wrong by me.

DC: Hmmm

TK: So, yeah, l'd say some of the meetings tend to be a little bit longer, sometimes 'cos of that...erm yeah. Yeah.

KP: I would say when I get a referral, I, I, I never look at the religion-

AHR: No

$\mathrm{KP}$ : -the religion doesn't bother me-

AHR: No 
KP: ... because my religion doesn't define who I am at all, I don't feel, so I don't...I very rarely look at the religion, I only tend to look at the religion when we, do we still have to fill it in on the statutory assessment?

AHR: Yeah

KP: We used to have to write it in and then I think "Oh! They're...!" And, sometimes people don't fill it in and sometimes I think "Oh, they're Christian!" That's funny, I didn't necessarily think they would be Christian...

AHR: Yeah

$\mathrm{KP}$ : ... because they come from $\mathrm{X}, \mathrm{Y}$ or $\mathrm{Z}$ but I, I never look at the religion.

AHR: I think there is more about the logistical things as to what this child's...

KP: Hmmm

AHR: .... where this child's been-

KP: It's more where they come from and the languages they speak...

AHR: Yeah [inaudible]

$\mathrm{KP}$ :...because whether I'm going to be able to and whether they speak good enough English- that's my biggest issue really, I suppose! If I will be able to communicate with them, or not.

MA: Okay. Again, just going back to what you said. That's interesting actually because I look at the religion, I will dress more conservatively than I would normally do.

KP: Really?

DC: How interesting!

MA: If I'm going to visit the parents, I'm thinking "Oh gosh! I've got to make sure..."

$\mathrm{KP}$ : Based on what?

MA: Well, basically if I find that the child's a Muslim, for example, and- 
DC: Yes

MA: -l've got to meet the parents as part of a consultation...

KP: Yes

MA: ...meeting, I will make sure I dress more conservatively that I usually do!

KP: So no bikinis!

[laughter]

KP: You always dress very conservative, anyway!

[talking all at once- inaudible]

MA: Just so the parents can relate to me better, I suppose.

KP: I never consider that.

MA: No?

KP: I always dress for work...

NA: Yeah

DC: Yeah

KP: ...or play. I don't wear my home, necessarily my home- necessarily my home clothes, unless I'm in the office all day. I, I-

TK: Do you mean that you might, instead of wearing a skirt, you might wear tr...

MA: Trousers or l'll make sure I wear a longer skirt or dress

KP: Yeah

TK: Yeah

NA: It's funny because that I...I w-dress for work too...

KP: Hmmm

NA: ....and then dress when I'm not working...

KP: Hmmm 
NA: ... but, the only time when I might think about what I'm wearing is if I knew I was visiting a Muslim family, I might when I'm putting my top on, just check that it wasn't too...

KP: [inaudible]

NA: ... but that's it. But, I mean 'cos what I'm wearing is not generally not gonna be...

AHR: You're probably more sensitive to it...

MA: Yes

$\mathrm{KP}: \mathrm{Hmmm}$

AHR: ...coming from that...

MA: I think having been a teacher previously as well and some children actually challenging me and say- in terms of saying “You can't be a Muslim because you wear [short] skirts...

AHR: Right!

MA: ...you wear [short] dresses" and I'm thinking "Oh gosh!"

KP: But do Muslims not wear skirts then?

MA: Well they do but this is talking about people from a very orth-

AHR: There're different cultures within...

TK: See that's very interesting!

KP: Because I've never been to a Muslim country...

TK: But...

KP: ....so I wouldn't know...

TK: Yeah...that-that question...

[talking all at once- inaudible]

TK: ...is really interesting because "Don't Muslims do this?" but, but we're a massive group of communities. 
KP: But that's...

TK: We have a huge variation...

$\mathrm{KP}$ : But that's one of the things

TK: Yeah...

$K P$ : ...we've said before that, I think $X[E P]$ said that she spoke to you about something about being a Muslim and I, I said the thing about religion is just because...you know, I probably wouldn't speak to you if it was something about being a Muslim because I presume that being a Muslim is such a big thing that just because you're a Muslim...

TK: Hmmm

KP: ... if you are, and you're a Muslim if you are, and X's [EP] a Muslim, you're views are going to be all so different...

TK: So different.

KP: So, I might get an opinion from you but it's not necessarily the same opinion as those parents and in fact they're... so, I probably wouldn't, necessarily ask. But I guess...

TK: For me then, it would be... it would be about, them having a just a different opinion...

KP: Yeah

TK: So, it might not be that my opinion is gonna be exactly the same as anybody else's. The same way that I could, I could, I would be thinking that "Oh" if I'd got a preschool case, I could, I maybe might go to someone that just has a little more experience.

KP: Hmmm

TK: I might completely disagree...

KP: Hmmm, yeah 
TK: ... with what you've said...

$\mathrm{KP}: \mathrm{Hmmm}$

TK: ... and your view on, on the- but I would still kind of just, just because l'd see it as a resource.

KP: But l'd almost...

[all talk at once: inaudible]

KP: I might've thought I was being a bit presumptuous just to presume you would know...

AHR: No, no, no, I think...

KP: ... what I mean...

[all talk at once: inaudible]

$\mathrm{KP}$ : Not that that was a criticism at all...

AHR: that I was telling them about what that...

KP: Yes

AHR: And, I was like "Oh Gosh! What do you think about that? And, like I say, I w- but another example of a non-Muslim example of this would be, erm, a Lithuanian child, when I met mum for statutory assessment and she said "As soon as he could walk, I left him to go to work for ten hours a day and that's acceptable in our culture!"

[laughter]

AHR: Right and this was through translation with a TA who was translating...

DC: [inaudible]

[laughter]

DC: Not on his own?

[laughter]

AHR: On. His. Own! 
TK: On his own?

AHR: For ten hours a day from the age of five until he went to school at seven she...

TK: Wha- At home with a granny?

MA: At home alone?

AHR: No, at home alone. And, she said to me "And that's culturally acceptable." And, as soon as she left the meeting and I was like, I was thinking "Ooh, is that culturally acceptable?" Well mum's, I don't know what Lithuanian culture is. Maybe it is culturally acceptable. I need to check that out. As soon as she walked out the room, the TA was like "It's not culturally acceptable, it's not what we do!" And, that boy's got very significant difficulties, possibly, largely, due to that....

TK: Wow.

AHR: ...But that- it was just so helpful to have her...

KP: Yes

AHR: 'Cos I probably would've just...I was thinking it...surely that can't be culturally acceptable but I need to check it out...

DC: Even if it is...

KP: Yeah

DC: ....it's still...

[all talk at one: inaudible]

KP: I saw a report from CAMHS the other day where there's a French family anand they- the boy had said that his mother's...

AHR: Sorry, that was in Lithuania she'd been...

KP: ...smacks him...

AHR: ...he had been left alone...

DC: Yeah 
$\mathrm{KP}:$...that his mother smacks him and they sp-spoke to the mother about, written this report to Mrs so-and-so about it is not acceptable to smack your son. And, she said "Ooh, she wasn't aware of that because it is in her country." France.

[laughter]

KP: It's amazing what parents can say, isn't it?!

AHR: That's great!

[laughter]

DC: There have been no prosecutions for FGM in this country, ever, have there? I mean...

NA: No

DC: Wha-that's, that is about people saying "Well actually that's a cultural or religious practise" and...

NA: Yes, yes, absolutely.

DC: ...I mean, I'm not intruding, and you know, these girls' are being abused in huge numbers.

MA: So, again it's that's diverse...

TK: That's due to exposure as well though, right?

DC: It is-it is but there must be I mean if, if not medication wise, er medically...[inaudible]

AHR: But how much more powerful...

DC: ...you know, in-in-in midwives and...

TK: Hmmm

DC: ... booking in for a you know...in...

TK: Hmmm

DC: People are seeing this and not doing anything about it. 
AHR: But don't you think it's much more powerful to have someone from that background...

$\mathrm{KP}: \mathrm{Hmmm}, \mathrm{hmmm}$

AHR: ...even perhaps from that country...

DC: Oh yeah

AHR: ....wherever it's happening...

$\mathrm{KP}: \mathrm{Hmmm}$

DC: Yeah

AHR: ...erm, that particular, a particular case to be advocating...

KP: Yes

AHR: ...that it's not culturally acceptable...

DC: Of course...

AHR: ... rather than us saying well, I read into it and...

DC: Of course

AHR: ... but for them, to, I just think there is something and that's what I was saying like, kind of th-

KP: Yes, I would never challenge...

LW: Yeah

KP: ... If somebody has said to me, from a different culture, "This is what we do in our culture" I would never turn round and challenge it. I would have to go back and do a bit of research to check it out.

TK: Check it out

$\mathrm{KP}$ : Absolutely.

MA: Would you feel comfortable challenging them then?

TK: I was thinking I might though, a little bit... 
KP: Yeah

DC: $\mathrm{Hmmm}$

NA: Like what though...

KP: Like what?

TK: I gu-I guess I need an example of it...

KP: Well there was this...

TK: I guess one of the things for me is that if it just feels wrong in the gut... KP: Yes...

TK: ... you should kind ach-not challenge, I won't be like "Well, you know, where?" but just "How does, what does that look like, where does that $d-d-d . .$. you know, how... is that helpful, why does, why does that work for your family?" In that way...

$\mathrm{KP}: \mathrm{Hmmm}$

TK: ...not to say 'Well no, in this country, it's, you can't, or...not to do that... $\mathrm{KP}: \mathrm{Hmmm}$

TK: ... because that would be 'them' and 'us' kinda situation KP: Hmmm

TK: ...but I would wanna, explore, if it felt wrong, so I can't kind of say, use that as an example...

KP: I remember a Filipino lady was telling me about how, erm, and she had two children that were Autistic, she'd married an English guy from Feltham and they'd come back to this country and, and she said in the Philippines, they, err, when you have a baby, they think it's healthy for a baby to cry every day. The baby has to cry everyday 'cos crying's healthy and so you flick their heels, with your finger when they wake up to make them cry and that, she said she was a midwife in 
the Philippines and that's what they do with their babies... and [laughs], I thought that was a bit sad really, isn't it? But...

MA: Did you ask her more about that $\mathrm{K}$ or did you just...

KP: No, she, I just said “We don't...

MA: Do that here?

KP: ...believe that here.

AHR: What if there was a white, working class lady who said to you "Oh, I've heard from somebody who lives in the Philippines that I should flick my child's heel because it's good for them to cry, would you then challenge that, would you then challenge them, or would you be like...

$\mathrm{KP}$ : Well, of course I would challenge them and I...

AHR: Yeah...

KP: ... would say we don't do that here and I, I also said "I thought most babies cried every day, anyway"...

AHR: Yeah

KP: I mean, y-you know you're quite lucky if your baby doesn't cry at some point in the day...

NA: Hmmm

KP: ...realistically, so, erm, i-

DC: [inaudible]...

$\mathrm{KP}$ : [inaudible] [laughs]

DC: ...You know, throughout human history, there's huge cultural diversity and how we bring up kids...

KP: Yes!

DC: ... and we think it's appropriate how we treat them...

[all talk at once: inaudible] 
KP: Have you seen that book? On Amazon there's a book being banned on Amazon at the moment or there's a massive petition. It's called "How to make a father love his child" or something and it's about these...ways of managing children's behaviour. It was written about fourty-odd years ago, I think, and, erm, it says you need to...make, er...I don't know, if you look on Amazon there's-it's just the most hideous book about beating children, about hurting them and about making them cry and, it's o- but there's quite a big fob-following for it...so... DC: We all think where we are, it's a linear view of history, isn't it... KP: Hmmm DC: ... where we always believe we're making progress and moving on and we've reached this apogee and...

KP: Hmmm

TK: Far from it!

DC: In fifty years' time or a year's time, we'll be saying different things about what's appropriate in relationships and, you know, I think you have to have a bit of humility about that don't we really...

NA: Hmmm

DC: ...so, it's not just our way of doing it is necessarily the only way but I think you're absolutely right, but it is easy for you [pointing to $\mathrm{TK}$ ] to be able to say "Well, can we explore that a bit, tell me a bit about...why you think flicking your baby's heels..." [laughs]

MA: Is it?

NA: Why is it easier for her?

DC: Well, I don't know, you're... if you're from a similar background maybe, do you not think they might...

AHR: Do you think it might make it less judgemental... 
DC: Yeah

AHR: ... and a bit more inquisitive?

TK: But, I'm only similar to a certain group, right?

DC: Yeah

TK: Really, on some level, maybe...

DC: Yes

TK: ...or not, so actually, if it was, if it was a Philipino mum...

KP: [to DC] and she wasn't...

DC: No, no.

TK: I wouldn't have anything similar so it would...

DC: No, no, no.

TK: ...Yeah.

AHR: I think because sometimes, when I'm presented with a cultural issue like that, I'm baffled and I'm thinking "I'm sure that's not right... but maybe it is!" I don't wanna offend them.

KP: Ummm [inaudible]

AHR: We wanna be productive in this...

$\mathrm{KP}: \mathrm{Hmmm}$

AHR: ....in this environment and consultation...

KP: Yeah

AHR: ... and we're trying to be productive. I don't want to, like, delve too deeply and offend them on their, about their culture...

DC: Hmmm

AHR: That's my issue, I suppose and I need to become more comfortable with asking naïve questions. 
MA: When you are faced with some of these sweeping statements, such as, you know, "We do that in our culture", have any of you ever used your psychology to kind of like, challenge or overcome that a little bit or, get the parent, person or guardian to ever...

DC: That's what T is saying, isn't it, that's exactly what you're doing isn't it?

TK: Hmmm...well, trying.

KP: Yeah and I think also with this Philippino lady, I think one of the reasons she told me... was to check it out to a certain extent...

TK: Hmmm

KP: I don't think she would've said...

TK: To validate it...

KP: ...You know, her children were older so she didn't do it anymore but she was telling me and, erm, I, I don't think she was...she wasn't challenging me about it. She was just saying and I think she was partly saying it to show that she knows it's not right, you know we don't do that?

DC: $\mathrm{Hmmm}$

KP: A-we, you know, in this country but it's how, she was explaining to me about the difference about the Philippines and the schools they went to and how you have to pay and it's all different. Erm, so I think, I think it is really useful and when you think about psychology about when people do give you those things, they tell you those things, I mean, some of them, the ones I, I've got more recently, or some things I think about is when parents say to you- I had an older dad and hethey're white, British and he said to me and he's got a younger child and an older child and... you know the younger child's possibly got ADHD and he said "Yeah, in my day, you just gave them a smack but you don't do that now, do you?" And, that's sort of the same, isn't it? 
NA: Hmmm

KP: They're, and, you know, you used to have to say "Well, no, no, we don't!"

TK: [laughs]

KP: And, sometimes I explain to them why we don't smack our children, why, you

know, it's not illegal but, you know, there are reasons why smacking isn't...isn't

accepted, erm, you to helping people understand.

DC: [inaudible]

MA: Thank you everyone, it's been really interesting... [debrief] 


\section{Appendix XI}

Six Phases of Thematic Analysis

Familiarising yourself with your data:

Generating Coding interesting features of the data in a systematic fashion across the entire data set, initial codes: collating data relevant to each code.

Searching for themes:

Reviewing themes:

Defining and naming themes:

Producing the report:

Transcribing data (if necessary), reading and re-reading the data, noting down initial ideas.

Collating codes into potential themes, gathering all data relevant to each potential theme.

Checking in the themes work in relation to the coded extracts (Level 1) and the entire data set (Level 2), generating a thematic 'map' of the analysis.

Ongoing analysis to refine the specifics of each theme, and the overall story the analysis tells; generating clear definitions and names for each theme.

The final opportunity for analysis. Selection of vivid, compelling extract examples, final analysis of selected extracts, relating back of the analysis to the research question and literature, producing a scholarly report of the analysis. 


\section{Appendix XII}

Summary of the Initial Codes, Developed Themes and Main Themes identified for Focus Groups 1, 2 and 3

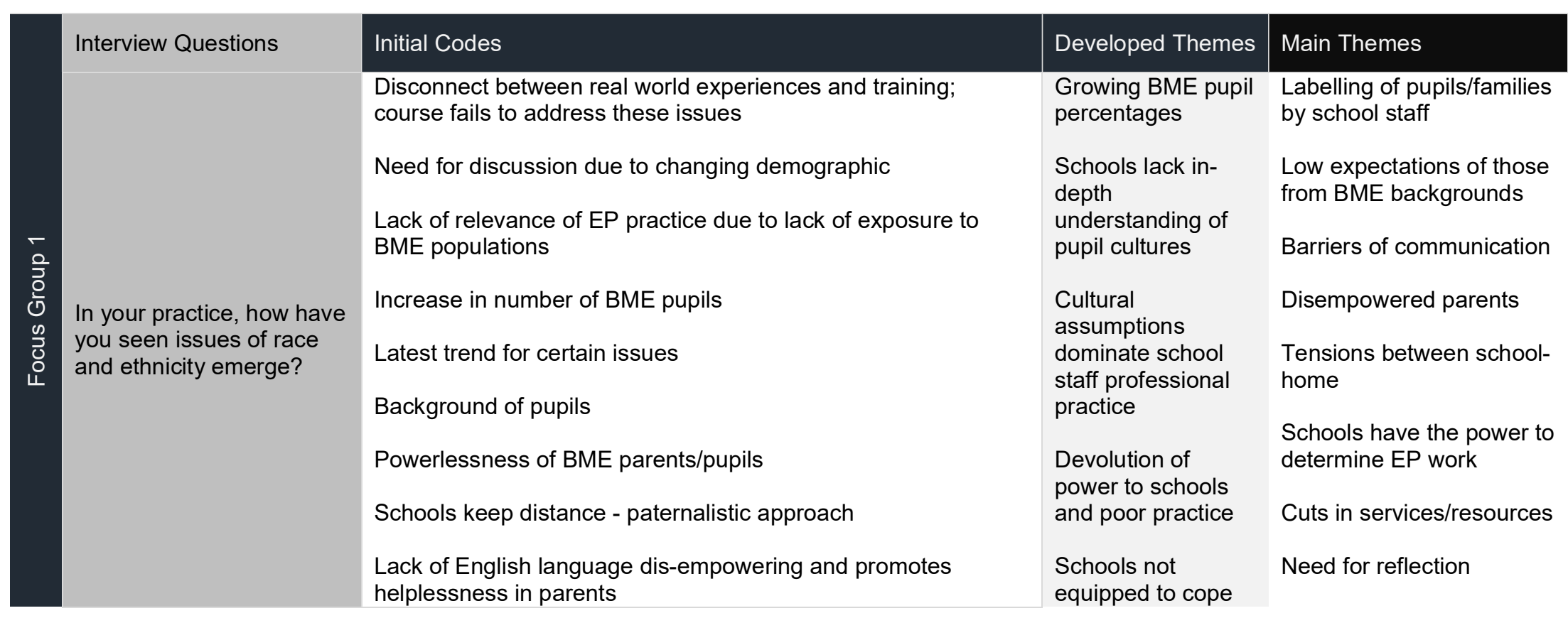




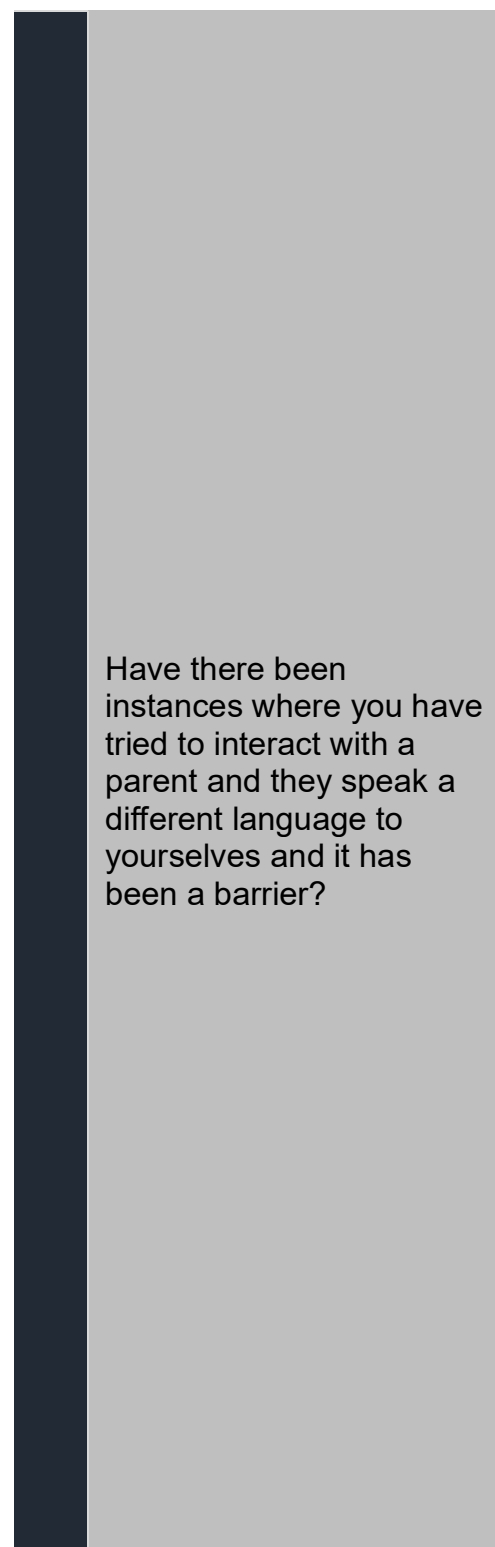

Arranging of a translator/interpreter lengthy process

Change in attitude from council

BME not on top of the agenda

Decentralisation of BME teachers

Removal of EAL teachers from schools - no longer important

Reliance on schools to provide interpreters; lack of

independence as using internal resources

Inconsistency between different schools/boroughs

Lack of investment in resources to match needs

Women subject to domestic violence

Women not empowered

Language linked to distress/violence

Role of EPs with Parents

Fear of reporting domestic violence leaves exposed

Unprivileged demographic

Assessments lacking due to additional variables

Assessments lacking due to limitations of tools

Information gathering, willingness of divulgence from parents and language barriers also limit assessments

Limitations of language development (EAL)

Importance of consulting with EPs and individual assessments (+working with parents)

Teachers make assumptions about children based on their own views (without consultations)
BME parents under-empowered

Language and translation barriers due to service cuts

Variance in RCE issues in different schools/catchment areas

EPs play role in bridging parent-

teacher gap

Tensions between school-home

EPs encourage schools/parents to take ownership

EPs need to challenge schoolparents

Need for trusting relationships

between EP school staff and parents

Can be emotionally taxing- need for reflection

RCE are controversial topics for discussion
Changing nature of work and 'clients'

EP training courses limit discussions/exposure to diverse populations 
Teachers' ideas vs parents' ideas - causes tensions

Challenging working with parents via interpreter
Accurate

assessments of

BME pupils lacking

due to limitations of

tools

Reflection and

awareness of own

beliefs important

Lack of investment

in resources to

match needs

Services

competing for

"clients"

\section{Information}

gathering hindered

due to language

Challenges of

using interpreters 


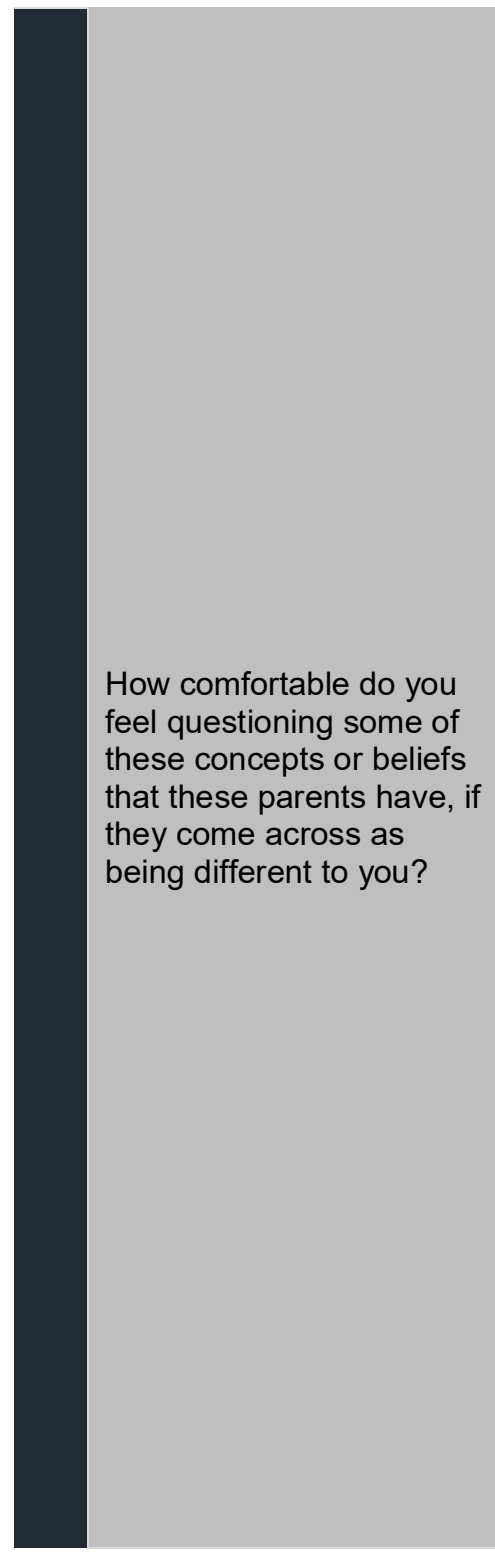

Confident and comfortable - crucial part of assessment

Important to respect parents' point of view

Important to reach middle ground

EPs not pro-active in research gathering; relying on parents to take the lead; inconsistent

Cultural factors not considered in reports

Importance of cultural factors only understood as a result of experiences and individual reading

Importance of knowing the background of the children that need assessing

Schools referring to incorrect body due to lack of background knowledge

Lack of awareness in schools around diversity/ethnicity

Inconsistency of awareness between boroughs

No conversation about cultural factors in schools, negative attitude towards subjects such as EAL's

Schools desensitised to issues (overwhelming)

Schools not taking into account consequences of not addressing issues

Individual reflection and awareness of own beliefs important seeking knowledge and questioning "truths"

There should be balance; don't over involve in RCE, so that EPs avoid danger of moving children from one category to another

Important not to ignore requirements of children and parents regardless of background 


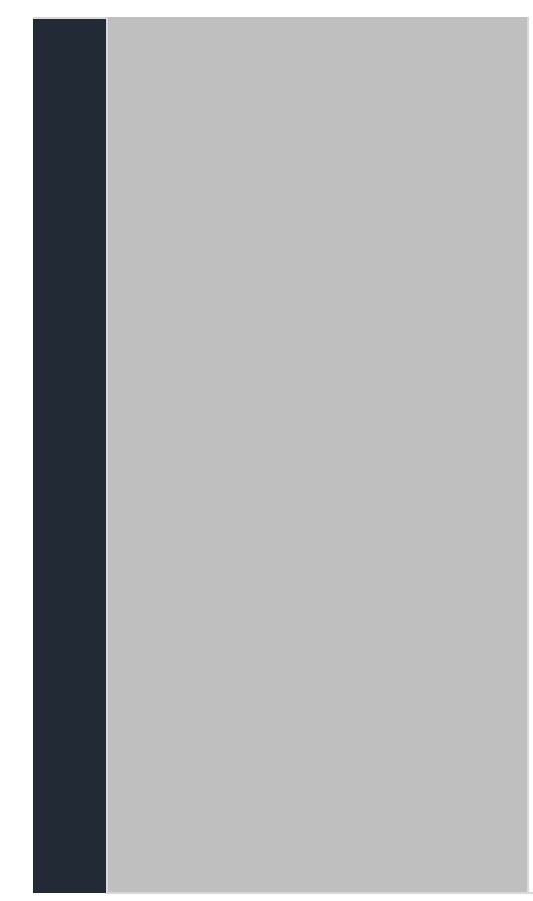

Schools sometimes take stereotypical view of what BME families are like

Best approach is to have very high expectations for every child

Importance of training - how to use language appropriately 


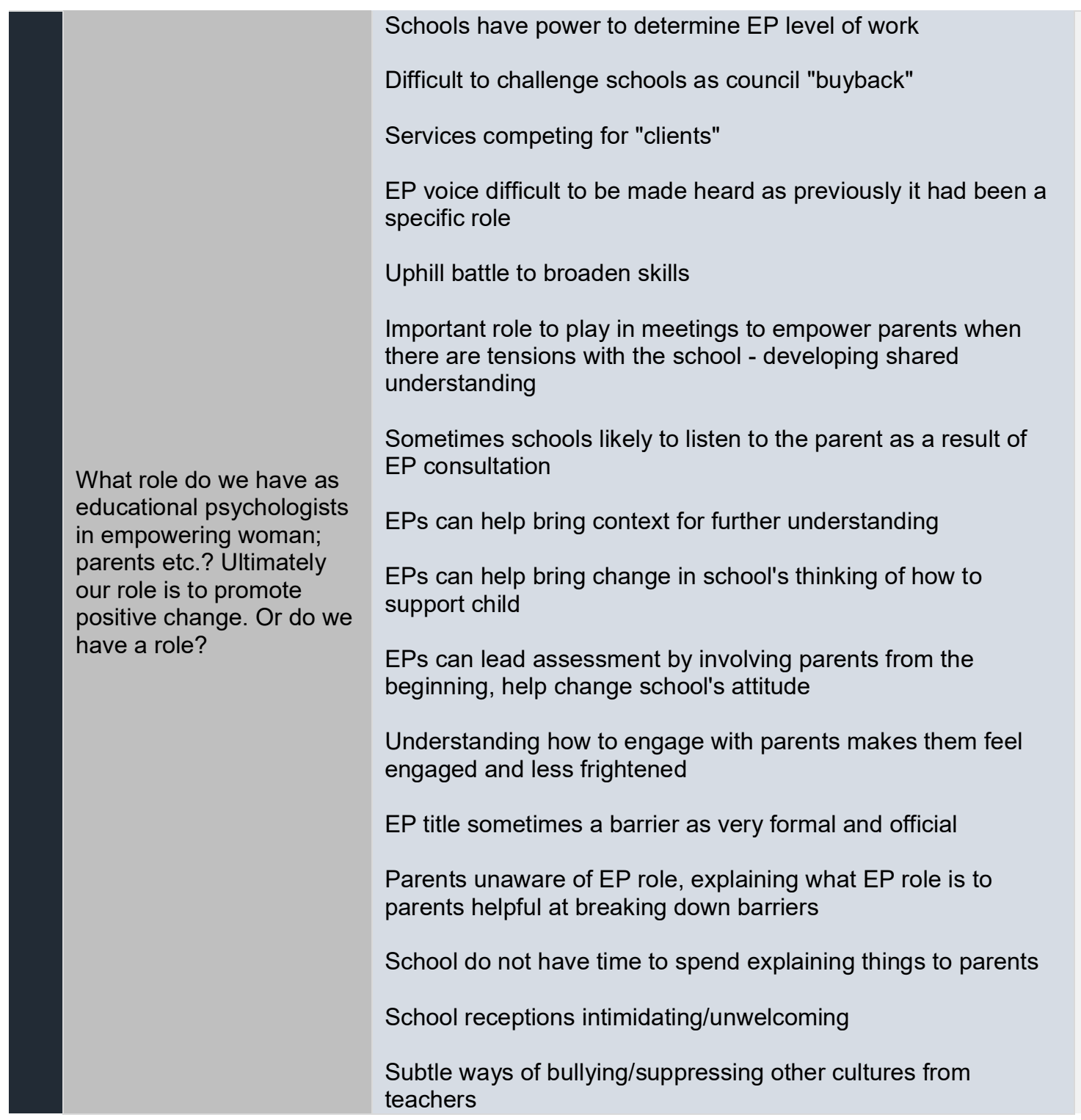


Negative attitude of schools towards BME parents/children

School administrative staff abuse power to keep parents "in line" and make them feel inferior

Ways of challenging things in schools

Schools mimic and perpetuate current world views

Building trusting relationships with schools

Race uncomfortable for people to discuss; controversial

Do EPs have a

responsibility to challenge

and neutralise the

zeitgeist in schools and

how it's coming across

towards parents and

children that might be

from the background

being vilified in the media?

Supervision doesn't address school attitude towards race

EPs feel unsupported from LA to challenge schools/staff

Overuse of psychometrics for assessments

Schools don't want anything other than psychometrics

EPs will try to convince schools to no use psychometrics solely

EPs would like more authority over consultations, rather than use of statements/tests 
How would you assess a child which has language delays if it is an assessment heavy borough?

\section{How easy is it to}

disseminate assessment information that you've

collected from the

CYB/parent to all involved, bearing in mind that

people are from different

backgrounds; have limited

understanding of

psychology in practice:

find the EP title scary?

Have there ever been

times when your own RCE

has affected your

experience of working with

parents, teachers, children

and young people? How

does it come across being

Caucasian when working

with parents who are not
Crucial to identify speech/language delay to stop impairment of development

Need for good practitioner for children with EAL and speech delays - least likely recognised

Consultation vs assessment approach - former is what is needed, latter restrictive

Important to use multiple tools to gather information from the child, parent and the school; however, tools are limited

Crucial to identify needs of child; working with an open mind; listening to concerns; not judging

Important to educate schools about which information they should gather as a minimum

Inconsistency within schools providing basic information

Schools get conflicting advice; difficult for schools as well

Lack of capacity to give proper feedback to parents

Providing thorough feedback to the school and parents is time consuming

\section{EP doesn't think about it - treats people as people}

Assumptions are made when parents are from similar background to EP

Important to take into account parents view point when SENCo and EP are from a different background; tone of language, eye contact crucial 
from your own

culture/ethnic

backgrounds?

Assumptions are made determining what makes people comfortable

Assumptions are based on experience, important not let them influence decisions

Parents generally want to share information; women co-operate

Working with a translator who is from the same culture as the

Does being the same parent, often results in less information being shared; don't want to share with community

ethnicity as the client give

you richer information?

Does it make your job

Showing politeness and engaging the parents/school will ensure better results

easier? Is there more trust

in that relationship?

Empowering parents enables them to make good decisions about their children

EP role is to demystify what parents think about special educational needs 


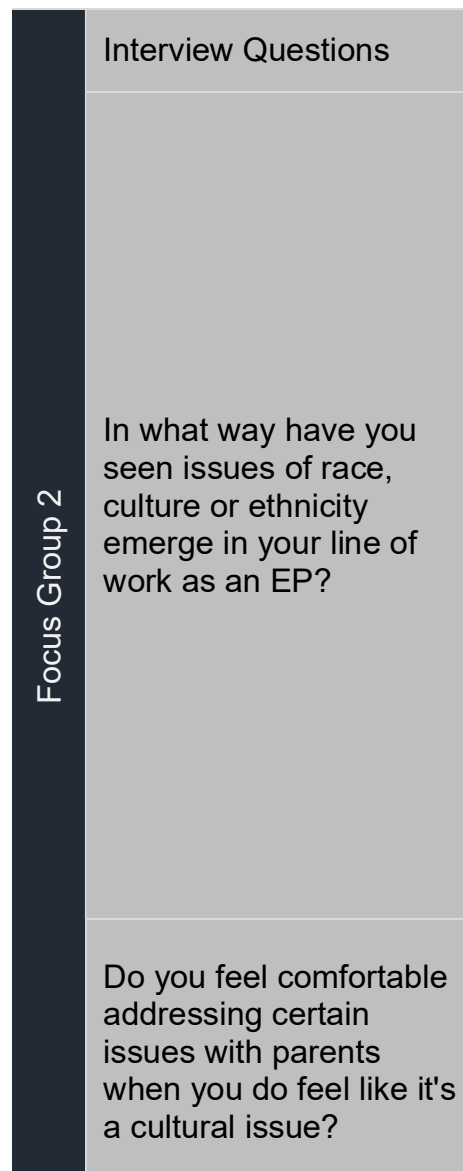

\section{Initial Codes}

Difference in attitude between what is considered learning between cultures; play not considered to be learning

EP explained benefits of play; learning process of a child, etc.

Few students from BME that EP work with

Historical issues with working with academies; perceptions of LEA services is a barrier

Schools that value special needs buy more EP services

Not all schools are getting full quality EP services

In borough children more likely to benefit from special needs services

Schools use service to get a specific diagnosis; narrowing use of EP

Expectations of parents from different backgrounds cause children to increase anxiety

Importance of child's status > cultural aspect of basis for status

Role of EPs in school include challenging of cultural stereotypes/expectations; role of a critical friend

Buyback option for schools making it difficult to preserve EP/school relations; EPs need to tread more carefully
Developed Themes

Main Themes

Increase in faith schools, Schools rigid and

free schools and

academies - not

representative of diverse society

Changing nature of education- focus on success not emotional well-being

Certain concepts may not exist (e.g. ADHD, autism) in other languages or labelled differently

Challenge cultural stereotypes/expectations in schools

Building rapport and forming positive relationships between home-school

Initiating/instigating difficult conversations unwilling to change

Results take priority over emotional health and well-being

Language barriers and difficulties disseminating technical information

Challenges of using interpreters

Positive relationships and rapport

Approachable,

supportive and nonjudgemental practice

Differing attitudes amongst BME parents re: learning and achievement

Unequal access to resources 
How do you feel when you come across someone who is very different to yourself, in terms of understanding what their needs might be and what they might require?

\section{Have you ever come} across school teachers, SENCos, who have made sweeping judgements, sweeping statements about a group of children or their families?

Do stakeholders only refer children that they perceive would benefit from some kind of intervention? How do you promote equality of access? What do EPs need to do?
Focus is on role and how work needs to be undertaken and what the aims are. Sometimes aim is to build rapport and forming a relationship. Sometimes situation means EP does not know how to respond (when faced with BME CYP); EP role is central to consultation

Children EP works with have a different school experience to $E P$, not much relevance placed compared to themselves

Looking at whole profile of what child's complex needs, giving consideration to background, but not focussing

Role is to piece together information; from school, family, home; assumptions from schools sometimes

School staff room culture - Chinese whispers about issues;

assumptions and statements made about children - unhelpfu - schools need to be challenged. Role of school is now the client, rather than the child being the client

Teachers not necessarily open or honest and raise their "game" when being observed by EP

EP role to challenge comments made by teachers regarding children from particular backgrounds

Duty of EP to challenge and have difficult conversations

Risk that referrals are made based on traditional view; statutory assessments valued by schools - system unequal

Role of EP to promote equal ops; difficult with current system fear privatisation would make system less inclusive

Role of EP to gather evidence and be clear in our mind -

when schools buy into psychology, snowball effect

Less about ethnic background, more about wealth - parents

that can afford more, will have more resources
Being accessible

Language barriers prevent thorough assessment

Different attitudes re: learning and achievement amongst cultures

Wealth dictates access to resources, not RCE background

Parents lack of trust for EPs

Variance in valuing SEN in schools

Traded services means schools- not pupils- are clients

EP capacity for in-depth work with

schools/families limited

EPs under-valued and role diminished

Changes in education and the diversity of the
Schools determine EP involvement

Traded services

Time constraints

Changing nature of EP work

EP Training courses need updating re: $\mathrm{RCE}$ 
Did you all train in London? Do you feel

that the curriculum of the course prepared you to work with a diverse range of people?

What roles do EPs have in meeting the needs of significantly underachieving boys?

How can EPs work with schools to disseminate and add substance to the figures? (about expectations)
Course director wanted to provide exposure/experiences in different boroughs

Fulfilling statutory function > training experience; first

experience of BME engagement was on placement

Parents don't always trust EPs from different backgrounds as they feel they won't understand

A surplus in the service would allow families/children who don't have access would be more effectively targeted Course requirement in year 1 to have BME as part of case work at one institution; another institution the priority was working with people from different backgrounds

Big emphasis on equal opportunities in the course from the outset - certain topics covered to help understanding

Need for current information; more difficult to scrutinise Free Schools and Academies; access determined by parent governors

Need to be responsive to local requirements, using up to date local data

Pace of change in education directed Government not reflected in training experience or courses

Need for awareness of background, however, more important to deal with individual child needs

Data about certain groups can give false expectations of those groups; expectations for all groups should be high

Schools should have high expectations of all children

Working with schools informally would provide opportunity for more in depth working

Need for awareness of local population; addressing stigma of being referred to EPs of some BME backgrounds

Need for responsiveness and sensitive and providing clarity of EP role

Not always easy to be responsive, use of skills such as empathy and listening

Use of open questions and framing them according to the people EPs work with

Being mindful of language used and make reports accessible Cannot be responsive to all as limited to how many children EPs can work with; psychology language used

If EPs funding stopped, then only parents who can afford will use the services.

Government of the day doesn't value EPs, therefore we aren't school population not reflected in training

courses 


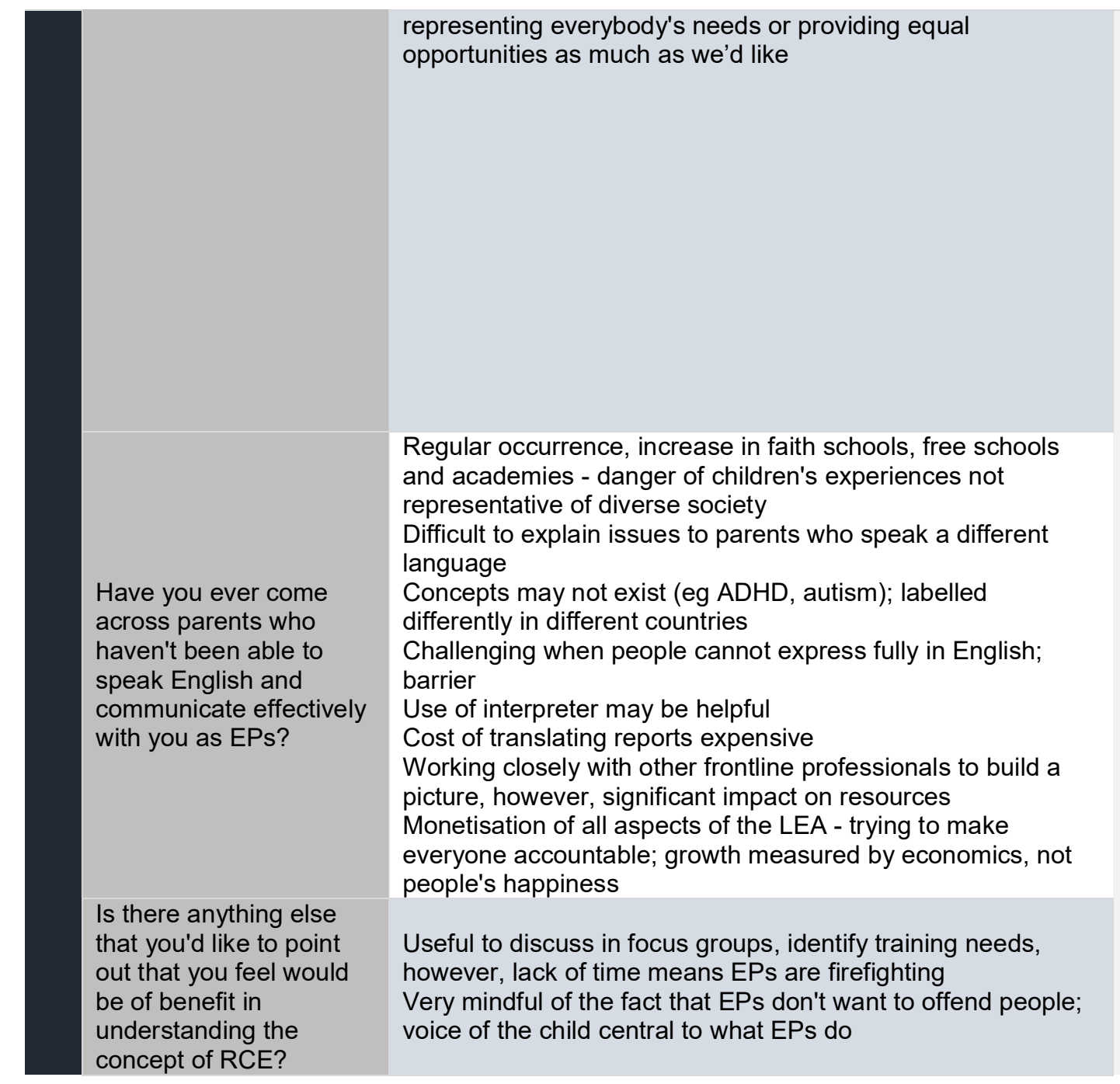




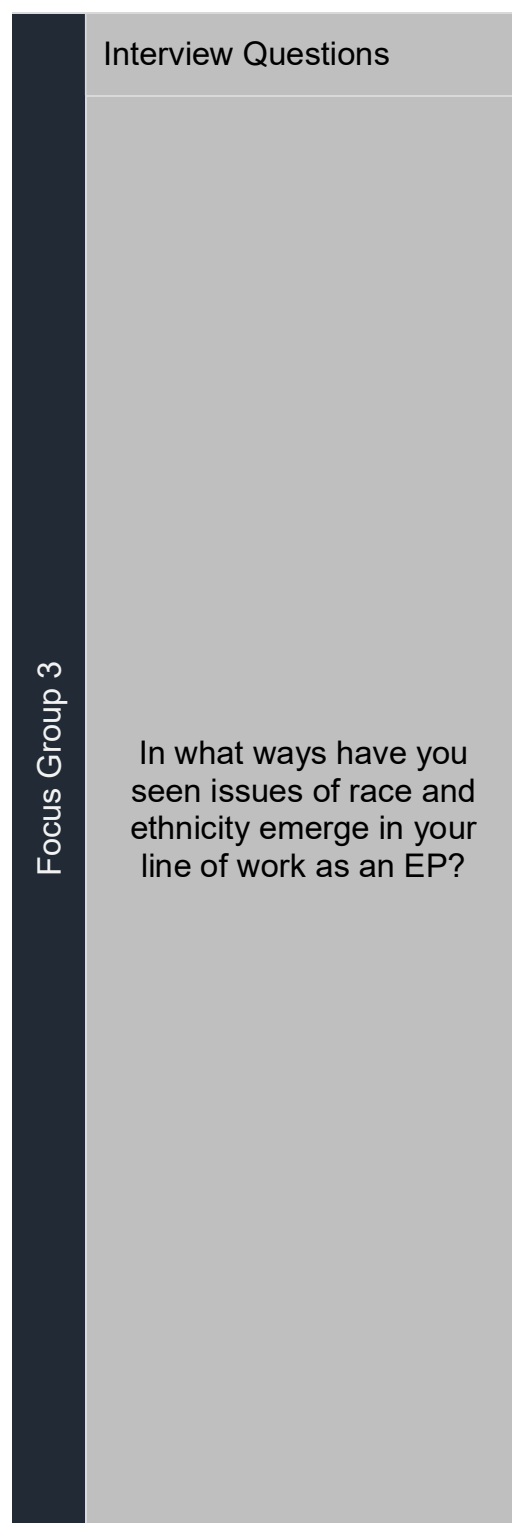

\section{Initial Codes}

Parents not taking issues seriously

Nothing available to backup

individual EP experiences

Unable to come to a conclusion

due to lack of backup

Stereotype of certain BME families exist

Some families don't play with

children based on cultural norm

EPs work with standard narrow models of what parenting should be

Should EP role be to step up,

reflect and challenge these narrow models?

SENCo's make broad judgements about parents based on their

background

Parents sometimes reluctant to

share viewpoints with the school

Non BME EPs unaware on how to

handle BME families; do not

always know where to find

answers

Parents not empowering children

by giving them relevant information

EP not comfortable with dealing
Developed Themes

Stereotypes of BME families exists within schools

BME assumptions driven by societal changes

Parents relate to EPs better if they look the same

CYP and BME families experience difficulties assimilating into new environments

Acceptable norms change over time

Uncomfortable dealing with BME issues with BME families

Lack of confidence

Open questions to check understanding

Cultural differences re: acceptable and unacceptable behaviours

Advocating for parents in schools

Challenges in relation to parents lack of English

Interpreters struggle to convey actual meaning

Interpreters is short supply

Rapport building compromised due to cultural restrictions
Main Themes

Cultural assumptions by school staff

Fear of offending

Questioning and clarifying

to ensure accuracy

Language barriers

Challenges of using interpreters

\section{Cuts in services}

Variation in cultural norms re: childrearing and education

Defensiveness from parents

Unequal access to EPs and other professionals

Importance of supervision

Lack of

confidence/experience re: RCE

Personal development and/or CPD

Intransigent EP theories and models

Outdated training courses 
with issue as not from same BME as family

Lack of BME EPs in LA

EPs should feel confident

discussing BME issues

Assumption that if EP is of same

skin colour, that they're from the

same background

EP from non-diverse background

takes time to feel comfortable with

multicultural area

RCE irrelevant as you may have huge differences with people from your own area

View of certain BME backgrounds driven by societal changes
Cultural differences in education and learning

Parents withhold information from professionals

School staff unfairly label parents based on their background labelling

Unequal access to EPs

Lack of BME EPs in LA

Differences in Supervision

Narrow models of parenting

NonBME EPs can feel confused working with BME families

Taking time to feel comfortable in multicultural areas

Lack of exposure when trained to CYP/families from BME backgrounds

Out of date assessments, tools and resources

Limited knowledge and/or understanding of diversity

EP RCE and parents

Stereotypes of BME families exists within for EPs

Importance of reflection

EPs non-representative of local population 


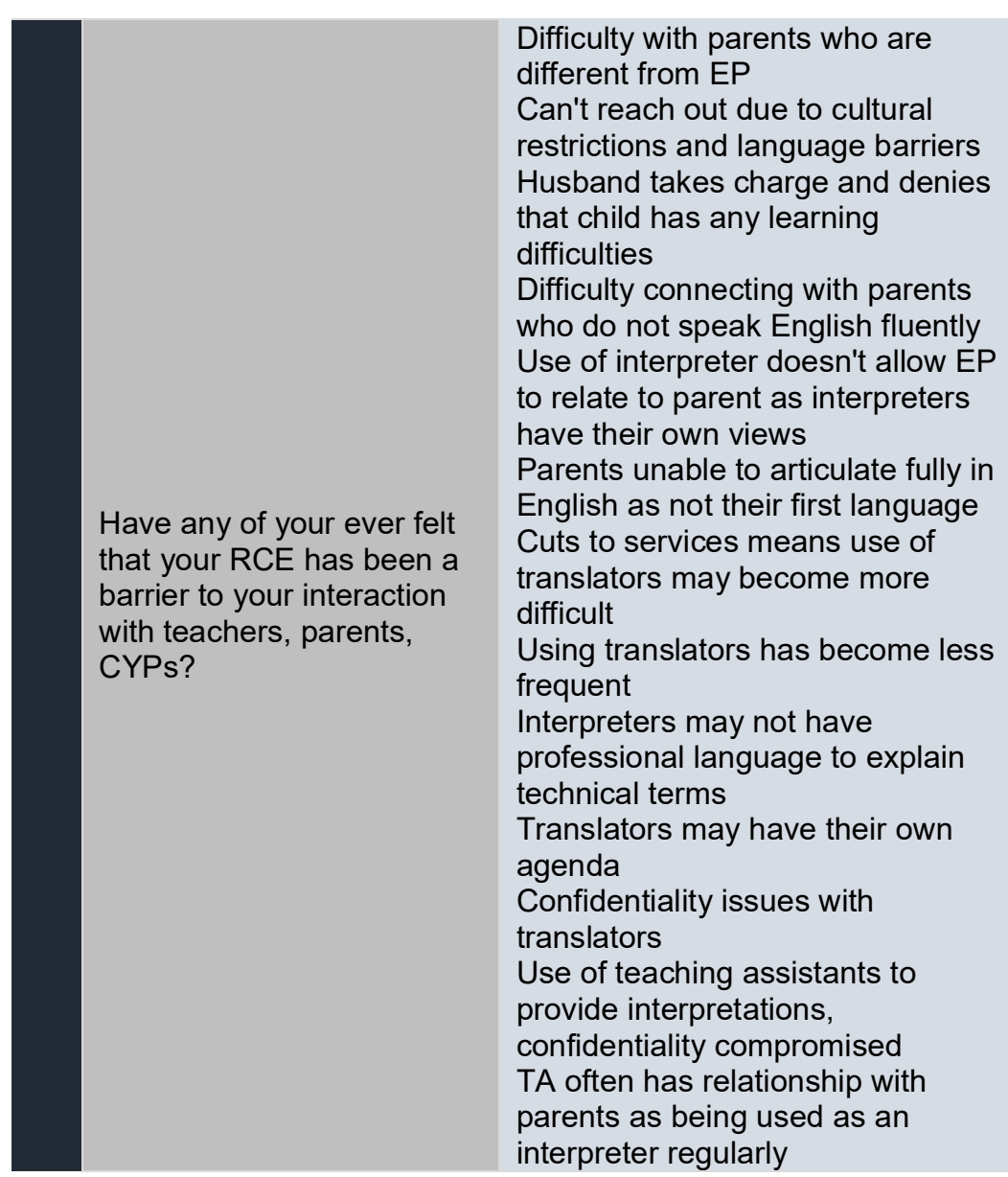

\section{schools}

BME assumptions driven by societal changes

Parents relate to EPs better if they look the same

CYP and BME families experience difficulties assimilating into new environments

Acceptable norms change over time

Uncomfortable dealing with BME issues with BME families

\section{Lack of confidence}

Open questions to check understanding

Cultural differences re: acceptable and unacceptable behaviours

Advocating for parents in schools

Challenges in relation to parents lack of English

Interpreters struggle to convey actual meaning 


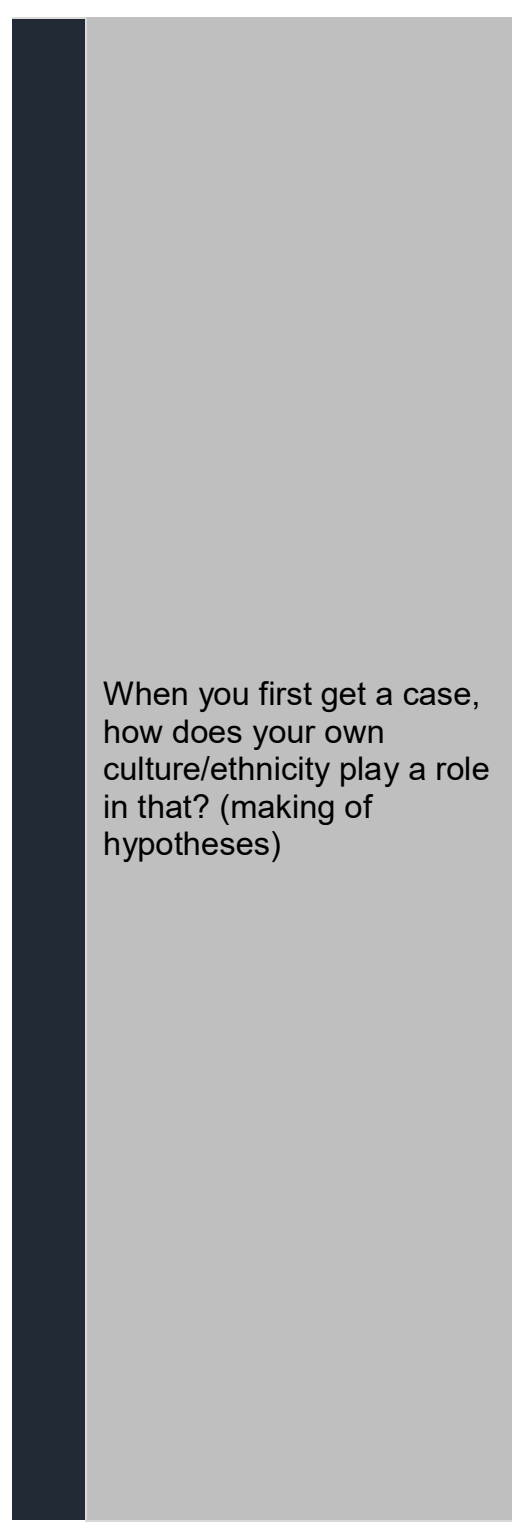

EP rarely was exposed to cases from a different background

Cognitive assessments on

bilingual students set out in

training book

Behaviour issue with children may be related to not being able to fit

into new surroundings

Schools not always asking the right questions of children from BME backgrounds

EP do not want to make

assumptions even if parents from own background as they may have

different ideals/beliefs

EP double checks that parents

identify with them based on

cultural similarities

Parents relay something in a

different but similar language to

that of an EP; EP then becomes

interpreter

Biggest issue for EP is whether

they can effectively communicate

with parents

Having to question whether what parent has said about their culture is true or not

Not easy to challenge parents about their culture as EPs don't

have enough background

\section{Interpreters is short supply}

Rapport building compromised due to cultural restrictions

Cultural differences in education and learning

Parents withhold information from professionals

School staff unfairly label parents based on their background labelling

Unequal access to EPs

Lack of BME EPs in LA

Differences in Supervision

Narrow models of parenting

NonBME EPs can feel confused working with BME families

Taking time to feel comfortable in multicultural areas

Lack of exposure when trained to CYP/families from BME backgrounds

Out of date assessments, tools and resources Limited knowledge and/or understanding of diversity

EP RCE and parents 
More likely to question someone

commenting on a culture that

wasn't their own

Acceptable norms change with

time

EPs don't want to offend by asking

questions 
When you are faced with

some sweeping statements

about cultural norms, have

you ever used your

psychology to challenge or

overcome?
Engaging the parents to discuss

the cultural differences and

explaining what is acceptable or 


\section{Appendix XIII}

Thematic Maps for Focus Groups 1, 2 and 3

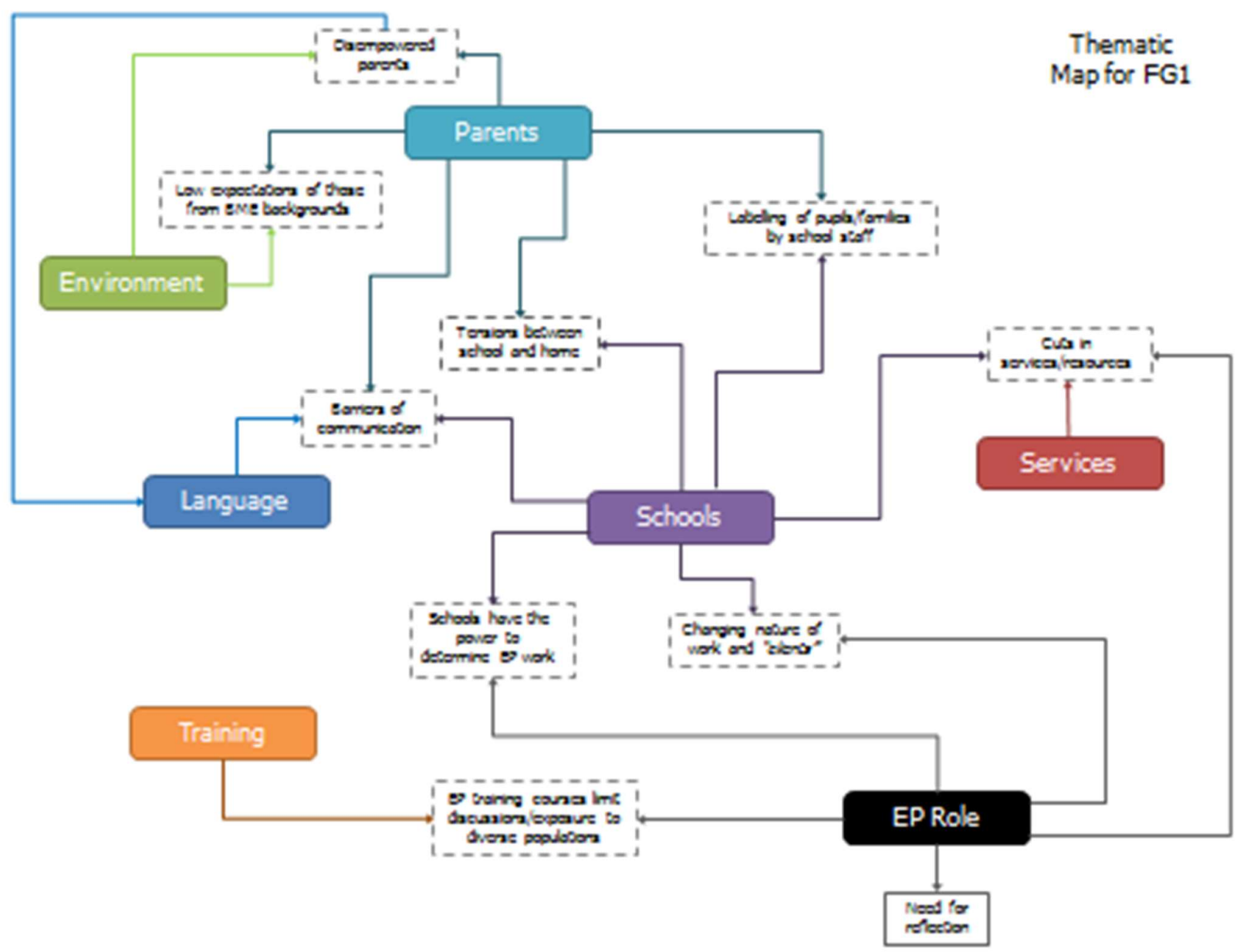




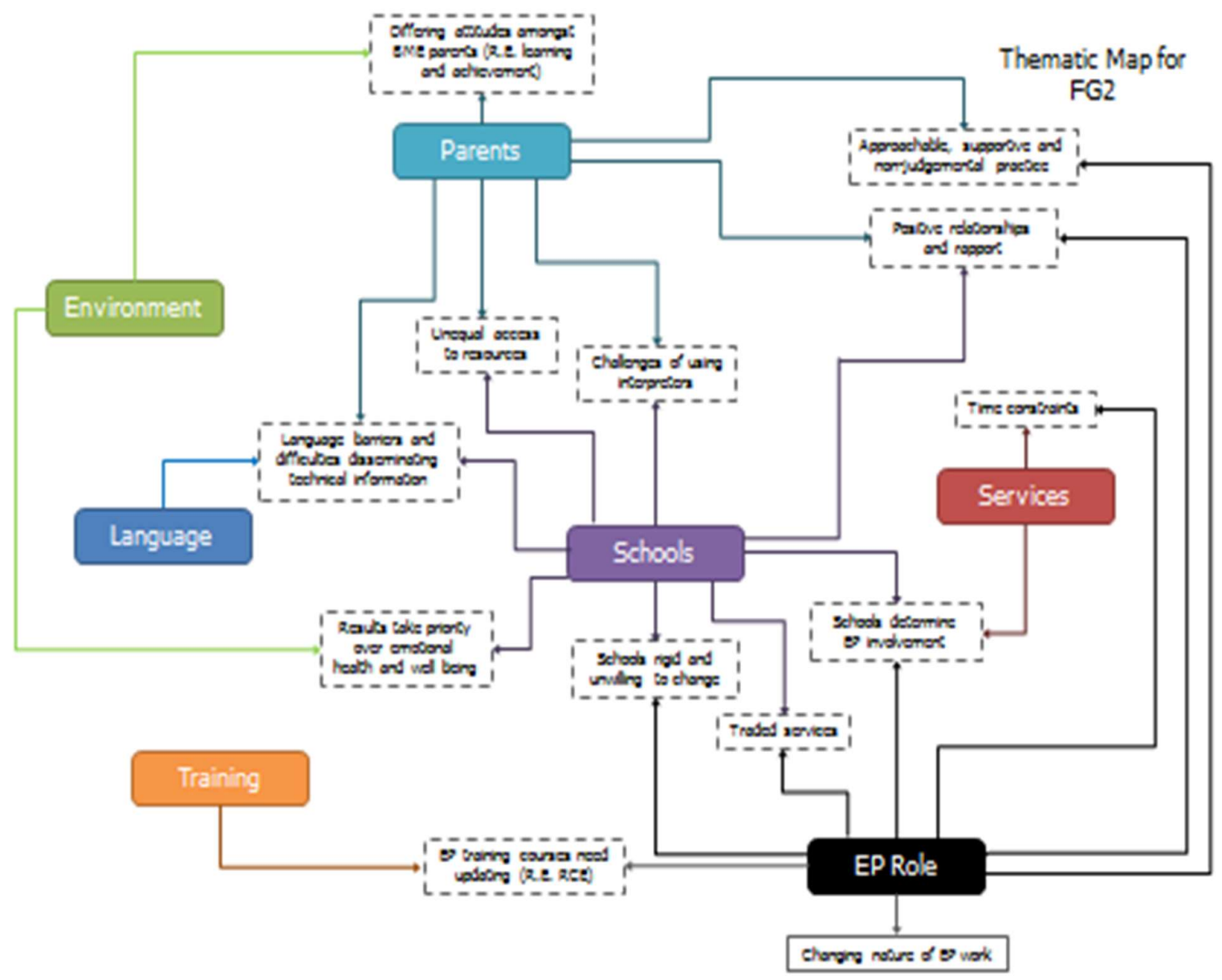


Thematic

Map for FG3

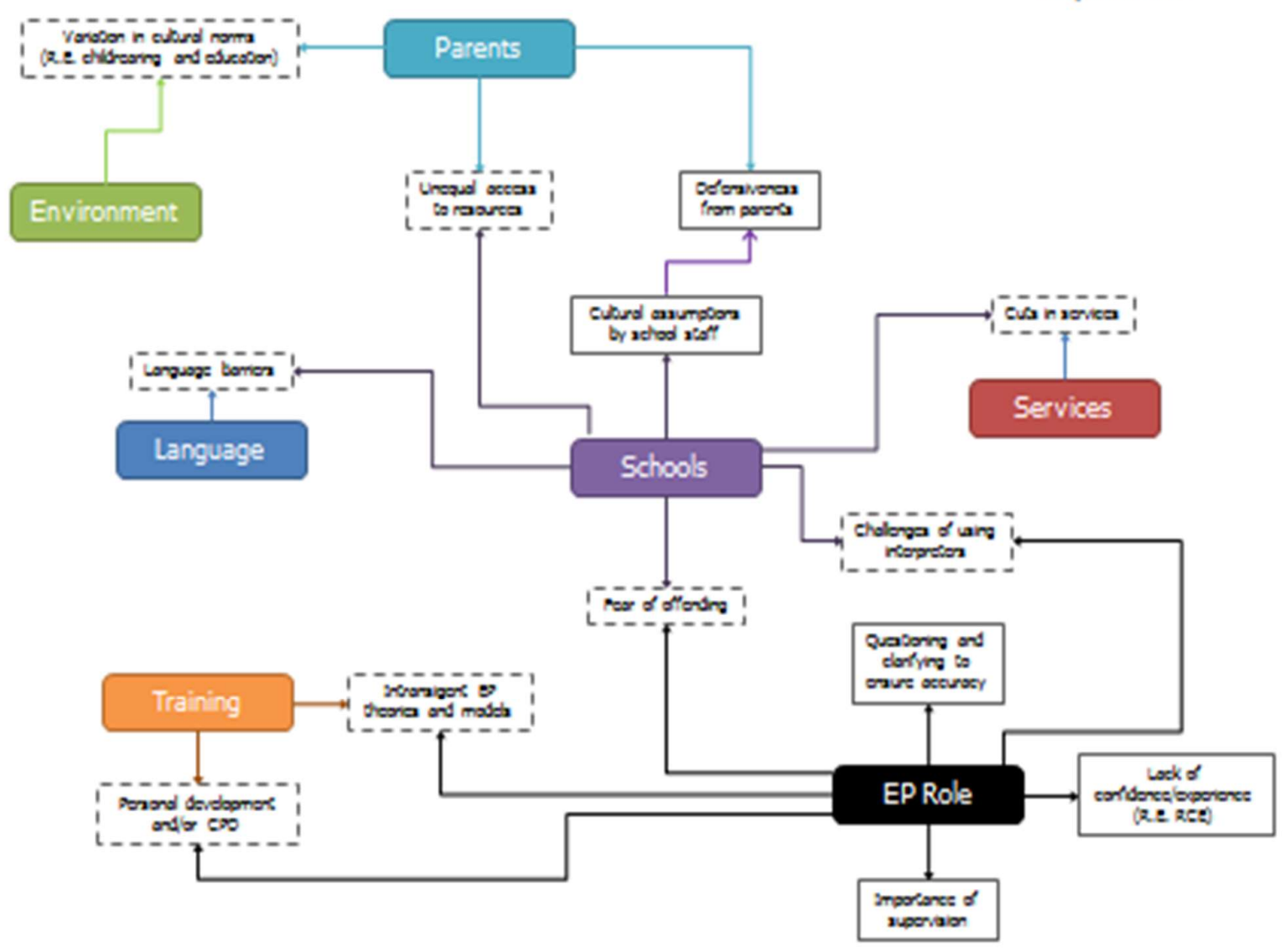

